\title{
InduCing NeUroplastic Changes in THE Human CORTEX USING EXTERnAL TransCRANIAL Electrical Stimulation TECHNiQUeS
}

\author{
Dissertation \\ zur Erlangung des mathematisch-naturwissenschaftlichen Doktorgrades \\ "Doctor rerum naturalium" \\ der Georg-August-Universität Göttingen
}

vorgelegt von

Leila Chaieb

aus Sousse, Tunesien

Göttingen, 2010 


\section{Doctoral Thesis Committee}

Prof. Dr. Dr. Andrea Antal (Supervisor, First Referree)

Abteilung Klinische Neurophysiologie

Universitätsmedizin Göttingen

Robert-Koch-Straße 40

37075 Göttingen

Prof. Dr. Tobias Moser (Reviewer)

Abtl. Hals-Nasen-Ohrenheilkunde

Robert-Koch-Str. 40

37075 Göttingen

Prof. Dr. Florentin Wörgötter (Reviewer)

Georg August University Göttingen

Bernstein Center for Computational Neuroscience

Friedrich-Hund Platz 1

37077 Göttingen

Date of Submission: $8^{\text {th }}$ October, 2010

Date of the oral examination: 


\section{Statement of Originality}

I hereby declare that this thesis is my own work and has been written independently with no other sources and aids than those quoted in the text, references and acknowledgements.

Göttingen, $8^{\text {th }}$ October, 2010

Leila Chaieb 


\section{Contents}

Chapter 1: Introduction ........................................................................................................... 1

1.1 Plasticity in the Central Nervous System ........................................................... 3

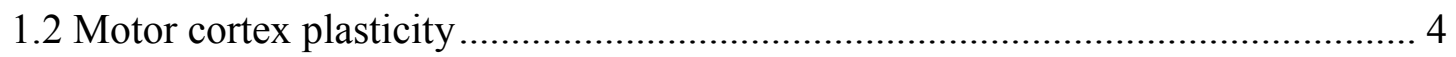

1.3 Non- invasive brain stimulation in humans .................................................... 5

1.3.1 Transcranial magnetic stimulation (TMS) …............................................... 5

1.3.2 Transcranial direct current stimulation (tDCS) ......................................... 7

1.3.3 Transcranial alternating current stimulation (tACS) ................................... 8

1.3.4 Transcranial Random Noise Stimulation (tRNS) ....................................... 9

1.4 Factors influencing mechanisms of neuroplastic induction: Brain derived neurotrophic factor (BDNF) and measures of cortical excitability .......................... 10

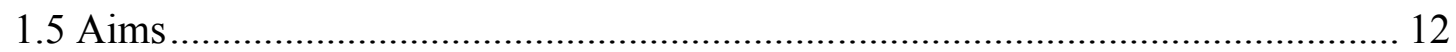

Chapter 2: Original Articles in the Thesis....................................................... 13

2.1 Increasing human brain excitability by transcranial high- frequency random noise

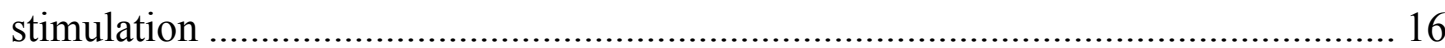

2.2 Short-duration transcranial random noise stimulation induces blood oxygenation level dependent response attenuation in the human motor cortex .

2.3 Evaluating Aftereffects of Transcranial Random Noise Stimulation on Cortical Excitability.....

2.4 Transcranial alternating current stimulation in the low $\mathrm{kHz}$ range increases motor cortex excitability

2.5 Comparatively weak after-effects of transcranial alternating current stimulation (tACS) on cortical excitability in humans

2.6 Brain-derived neurotrophic factor (BDNF) gene polymorphisms shape cortical

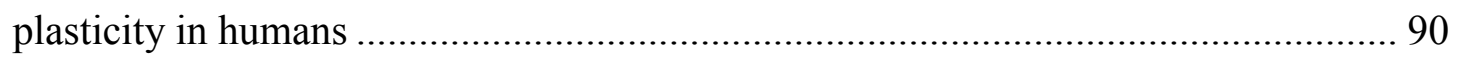

2.7 Frequency-dependent electrical stimulation of the visual cortex ........................ 99

2.8 Pergolide increases the efficacy of cathodal direct current stimulation to reduce the amplitude of laser-evoked potentials in humans

2.9 Gender-specific modulation of short-term neuroplasticity in the visual cortex induced by transcranial direct current stimulation.

Chapter 3: Discussion and Concluding Remarks .............................................. 125

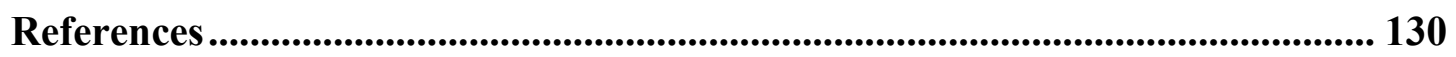

Acknowledgements ........................................................................................................ 135

Curriculum Vitae ........................................................................................................ 136 


\section{Chapter 1: Introduction}

Transcranial electrical and magnetic stimulation techniques have become increasingly popular methods, both in basic research and within a clinical setting, to stimulate targeted areas of the cerebral cortex painlessly, non- invasively. Since the development of non- invasive stimulation techniques with which to stimulate the intact human cortex, a wealth of studies have amassed a large amount of data suggesting that a wide variety of neurons can be targeted by different intensities and polarities of stimulation (Rothwell, 2005).

Stimulation of the intact human cortex was initially challenging due to the high resistance of the scalp and skull and by the naturally high threshold of the cortex itself. However, a seminal study conducted in 1980 by Merton and Morton, showed that by applying a small anode over the hand area of the motor cortex and an identical cathode over the vertex of the head and applying very short pulses of high intensity current which overcame the high resistance of the skull, electrical stimulation of the human motor cortex was entirely possible, if somewhat painful (Merton and Morton, 1980). This was major breakthrough for transcranial electrical stimulation techniques; soon other methods began to follow. Transcranial direct current stimulation (tDCS), the best characterized of these methods so far, was applied to the intact human cortex in 2000 by Nitsche and Paulus (Nitsche and Paulus, 2000). tACS (transcranial alternating current) and tRNS (transcranial random noise stimulation), two newer methods, have since emerged as promising tools in both basic and neurophysiological research, with the later aim of further optimizing these techniques to be useful as potential therapeutic measures, with a particular emphasis in pharmacologically intractable neurological disorders.

Transcranial magnetic stimulation (TMS) was the first clinically applied method, introduced in 1985 by Barker and colleagues (Barker et al., 1985) that has enabled the measurement of global levels of cortical excitability, and the evaluation of other interventional transcranial stimulation methods, when applied over the scalp to the 
superficial layers of the cerebral cortex, including remote areas connected via neuronal projections from the cortex. It also allows the integrity of the central motor pathways to be accurately assessed, lending it a useful role as diagnostic tool (Kobayashi and Pascual-Leone, 2003).

Now, such transcranial tools have been widely used to investigate processes of neuroplastic induction and their underlying mechanisms in the intact human cortex. Prior to the application of TMS, such investigations were only possible in animal preparations but with the advent of TMS and tDCS, it has become possible to induce cortical plasticity in the human cortex safely and reversibly.

This thesis will focus on evaluating the role of transcranial electrical stimulation techniques, how they are able to induce neuroplastic effects in the intact human cortex and their methodological approaches. The objectives of the dissertation are to explore the ways in which transcranially applied currents are able to induce neuroplastic aftereffects, their impact on neural networks, how long these aftereffects endure and how they can be modulated. This includes evaluating the stimulation parameters applied and how they can be optimized for future use within a clinical setting. Key studies highlight developments in novel methods of transcranial electrical stimulation; namely tACS and tRNS, and how applications of oscillating currents within specified stimulation parameters are able to impact upon neuroplasticity. A functional magnetic resonance imaging (fMRI) investigation will demonstrate that applied tRNS currents are subject to task-related modulations, and that application of tACS within the low $\mathrm{kHz}$ range can induce long-lasting changes in cortical excitability. Featured studies range from an analysis of gender roles in neuroplastic induction, the development of novel transcranial methods and their applications, and the impact of genetic variability on the ability to induce neuroplastic effects. Several other works detailing studies related to the thesis will provide additional information about the applications of specific transcranial electrical methods.

The aim of this thesis is to further our understanding of how neuroplastic effects can be induced within the human cortex using external stimulation methods, and how these 
techniques can be developed and optimized to induce long- lasting, measurable changes in cortical excitability which can then be observed as a manifestation of behavioural or cognitive changes. The further-reaching objective is to characterise approaches for tRNS and tACS that may be implemented as potential therapies for the symptoms of neurological disorders.

The following chapter will provide a brief introduction to the background of external stimulation techniques, how and why they are applied and their resultant effects. Chapter 2 constitutes the main body of the thesis detailing relevant studies and featuring published or submitted manuscripts currently under review. Each manuscript will have a foreword describing its relevance to the aims and objectives of the thesis. Finally, Chapter 3 will summarise the main findings of the studies conducted and place them into context within current knowledge, and will indicate future research directions.

\subsection{Plasticity in the Central Nervous System}

The brain's innate ability to remodel itself in response to external stimuli, which in turn modifies neural circuitry that impacts on future behaviours and cognitive processes, is termed neuroplasticity (Citri and Malenka, 2008). Plasticity occurs at different levels within the CNS and can modify brain networks by one of three mechanisms: by altering the efficacy or strength of synaptic transmission at preexisting synaptic terminals; by the growth/ sprouting of new synaptic connections or the trimming of existing ones; or by modulating the function of individual neurons (Malenka and Bear, 2004). Longterm potentiation (LTP) and long- term depression (LTD) are two specific forms of synaptic plasticity relating to the sustained increase or decrease in plasticity associated with changes in receptor expression, synaptic weight, and the increase or decrease in synaptic strength, and have been characterized at the glutamatergic synapse (Cooke and Bliss, 2006). Both forms have been studied across many species, mainly using in vitro slice preparation, with the hippocampus as a model (Bliss and Lomo, 1973, Cooke and Bliss, 2006). Mechanisms of LTP in the neocortex are strongly mediated by N- methylD- aspartate (NMDA) receptor activation and the alteration of the postsynaptic button to 
presynaptic neurotransmitter release (Feldman, 2009). While LTP is primarily an inputspecific process and is thought to underlie processes of learning and memory storage, it can be closely identified with the rules of Hebbian plasticity describing modifications in synaptic strength and efficacy between neural networks arising from the simultaneous activation of neurons in response to a repetitive input (Hebb, 1949). Such mechanisms are important and closely relate to maladaptive or functional neuroplastic changes, associated with use- dependent learning and task-repetition, within the cortex following traumatic brain injury or physiological alterations (Pascual-Leone et al., 2005).

\subsection{Motor cortex plasticity}

Many studies of neuroplasticity in the human cortex have been conducted using the primary motor cortex (M1) as a model due to the ease at which global levels of cortical excitability can be measured, i.e. by eliciting motoric responses like motor-evokedpotentials (MEPs). Although most processes of LTP and LTD have been characterized using the hippocampus, changes in synaptic plasticity have also been demonstrated in the mammalian motor cortex (Hess and Donoghue, 1994). Mechanisms of motor cortex plasticity are partly due to the dynamic cytoarchitecture of the M1 and the system of horizontal connections that spans the M1; this system of connections functionally attach M1 neurons to form neural networks that result in the construction of dynamic motor maps (for a review see (Sanes and Donoghue, 2000). These horizontal connections have been identified as candidate substrates for M1 plasticity due to the identification of occult motor maps in the M1 using local blockade of GABAergic inhibition that is able to unmask the horizontal connections usually blocked by feed-forward inhibition (Jacobs and Donoghue, 1991). It has also been stated that M1 organization depends upon the explicit balance between excitatory and inhibitory influences within the M1 (Sanes and Donoghue, 2000). Several studies have shown that M1 plasticity is functionally relevant to use- dependent learning (Cohen et al., 1998, Ziemann et al., 2001) and reorganization of neuronal networks to compensate for lack of function in areas damaged following brain injury (Butefisch, 2006). In addition, transient modifications in sensory-inputs (Brasil-Neto et al., 1992) and repetitive M1 stimulation 
(Berardelli et al., 1998) amongst other factors are all able to modify movement representations in the M1. The ability of the M1 to dynamically reorganize itself is of tremendous interest to the field of neurological rehabilitation (Duffau, 2006). The role of M1 plasticity is also fundamental to the processes of motor learning and memory consolidation (Ziemann et al., 1998b).

\subsection{Non- invasive brain stimulation in humans}

Advances in neuroimaging methods like positron emission tomography (PET), electroencephalography (EEG) and functional magnetic resonance imaging (fMRI) have enabled much research into understanding the link between cognitive processing and functional changes in cortical plasticity with a high spatial and temporal resolution. Transcranial electrical and magnetic techniques have been undergoing constant development, and have become useful tools in enabling us to elucidate how neuroplastic effects can be induced, monitored and sustained within the living cortex, as well as for therapeutic and diagnostic purposes. Concurrent use of these methods allows the study of immediate and enduring effects of interventional methods of transcranial electric and magnetic stimulation and lends an insight into their effects on neuronal activity (Siebner et al., 2003).

\subsubsection{Transcranial magnetic stimulation (TMS)}

TMS has emerged as a promising method not only within a clinical setting for the diagnosis of disorders of the nervous system, but as a powerful research tool. It has applications in disorders as far reaching as depression to Parkinson's disease, with studies reporting promising findings (Ridding and Rothwell, 2007). In a single- pulse monophasic application, its mode of action is to induce a secondary 'eddy' current within the brain; the result of a rapidly changing electrical current which is passed through a coil placed above the scalp. This generates a transient magnetic field that is able to pass through the skull and scalp unimpeded. The biophysical properties of the 
magnetic field; a fast rise time and slow fall off result in the induction of an electrical current within the conductive tissues of the targeted cortex. Neurons in the superficial layers of the cortex (usually when TMS is applied over an output cortex, namely the M1, or the visual cortex) are stimulated to discharge, propagating a volley of action potentials along the corticospinal tract. (Jalinous, 1991, Rothwell, 1997, Hallett, 2000). For ease of use TMS is usually applied over the M1, and used to measure MEPs elicited from the cortical representation of the small muscles of the hand; abductor digiti minimi (ADM), abductor pollicis brevis (APB) and first dorsal interosseous (FDI). Coil geometry, size and stimulus/ current orientation relative to the surface of the brain are all factors determining the focality of stimulation (Rossini et al., 1994, Ruohonen and Ilmoniemi, 1999). TMS preferentially evokes I-waves (indirect waves) at a lower intensity than D-waves (direct waves), in comparison to transcranial electrical stimulation (TES), which evokes D-waves initially at a low intensity, depending upon the direction of induced current flow. Epidural recordings show that TMS activates pyramidal tract neurons transsynaptically, whereas TES activates pyramidal tract neurons directly (Di Lazzaro et al., 1998, Rothwell, 2005). The application of a TMS pulse to the cortex may activate a variety of neurons within a population depending upon their orientation relative to the induced electric field; as a result TMS may give rise to both inhibitory and excitatory responses concurrently (Siebner and Rothwell, 2003, Huerta and Volpe, 2009). Single- pulse monophasic TMS is often applied to the M1 before and after intervention as the size and amplitude of elicited MEPs reflect changes in corticospinal excitability (Rothwell, 2005). Certain parameters are used to assess the excitability of the motor cortex in addition to MEP amplitudes; resting motor threshold (RMT) and active motor threshold (AMT) both reflect the level of excitation needed for motor neurons to reach discharge threshold during rest versus contraction, while motor threshold (MT) is defined as the lowest stimulus intensity required to evoke a measurable MEP (Rothwell, 2005).

TMS can be applied in a number of ways, not limited to the corticospinal tract. For example intracortical effects can be assessed using paired- pulse measures of excitability and are often used in conjunction with pharmacological agents in order to elucidate either the mechanism of TMS action, or to profile drug interventions (Kujirai 
et al., 1993, Ziemann, 2003). Input- output (I/O) recruitment curves can be used to identify cortical sub-populations responsible for inhibitory or excitatory effects and Iwaves to measure enduring aftereffects of interventional stimulations (Ziemann and Rothwell, 2000). Other highly specialized TMS protocols include theta- burst stimulation (TBS) paradigms; a subdivision of repetitive TMS (rTMS) which once applied over the M1 can achieve robust excitatory aftereffects lasting up to 30mins (Huang et al., 2005). Such highly specialized methods are useful in establishing novel therapeutic measures for neurological pathological conditions and in modulating cortical plasticity.

\subsubsection{Transcranial direct current stimulation (tDCS)}

tDCS was first applied in conjunction with TMS in order to evaluate neuroplastic aftereffects in the human cortex, in a seminal study by Nitsche and Paulus (Nitsche and Paulus, 2000). They observed that a short stimulation duration (13mins for anodal tDCS and 9mins for cathodal tDCS), at $1 \mathrm{~mA}$ was able to induce sustained excitability increases (anodal) or diminutions (cathodal) that outlasted the stimulation duration itself. A later study demonstrated that these alterations in excitability were able to be prolonged until 30mins poststimulation (Nitsche and Paulus, 2001, Nitsche et al., 2003). This significant finding has prompted many studies that have since applied tDCS to other brain cortices including the visual system (for a review see: (Antal and Paulus, 2008) and areas associated with cognitive processing (Reis et al., 2008). tDCS has emerged as a powerful tool in the treatment of chronic pain syndromes (Antal et al., 2010) and many other neurological disturbances (Nitsche and Paulus, 2009). Its mechanism of action is believed to underlie changes in neuronal membrane potentials, leading to alteration in intracellular cAMP levels and calcium influxes (Gartside, 1968, Islam et al., 1995). Applications of NMDA receptor antagonist dextromethorphan (DMO) have shown that tDCS neuroplasticity is NMDA receptor dependent, after having observed the complete blockade of all tDCS aftereffects post drug application (Liebetanz et al., 2002). tDCS is polarity, duration and intensity dependent and its aftereffects are subject to changes in the classic electrode montage; which is the 
placement of stimulation electrodes over the M1 and contralateral orbit, above the eyebrow (Nitsche and Paulus, 2000, Nitsche et al., 2005). Other studies also suggest the involvement of mechanisms other than membrane hyperpolarisation; synaptic effects (Purpura and McMurtry, 1965). However, these hypothesized modes of action are yet to be fully understood.

\subsubsection{Transcranial alternating current stimulation (tACS)}

The first observed effects of tACS on the human motor cortex were published by Antal and colleagues, who reported that the aftereffects of applied current at low intensities $(0.4 \mathrm{~mA})$ and low frequencies $(1,15,30$ and $45 \mathrm{~Hz})$ were negligible and transient; with only a significant effect of $10 \mathrm{~Hz}$ tACS over the M1 reported during the performance of the serial reaction time task (Antal et al., 2008). The 'comparatively weak' aftereffects were postulated to be a result of these two factors, however, later studies have since shown that application of higher frequencies over the motor cortex are much more tolerable than lower frequencies, due to the lack of interference of retinal stimulation from the electrode over the contralateral orbit (Chaieb et al., 2010). Recent studies also report inhibitory effects of tACS over the motor cortex at lower frequencies, of $15 \mathrm{~Hz}$, (Zaghi et al., 2010) and that phosphenes can be induced in a frequency-dependent manner in the visual cortex (Kanai et al., 2008). However, there is some controversy as to whether the perceived phosphenes are retinal or cortical in origin (for a commentary see (Paulus, 2010, Schutter and Hortensius, 2010). Pogosyan et al observed that $20 \mathrm{~Hz}$ tACS, an important intrinsic oscillatory frequency of the motor system, was able to slow voluntary movement. They postulated that the bursts of beta activity present in the cortex are correlated with the reinforcement of motoric responses after processing of sensory feedback during periods of tonic contraction. Therefore, the entrainment of networks in the motor cortex to the intrinsic $20 \mathrm{~Hz}$ frequency superimposed by tACS reduced the velocity of voluntary movement (Pogosyan et al., 2009). This study provides useful insights into the pathophysiology of Parkinson's disease, and why the motor system is inhibited by the predominant beta oscillating frequency found in patients. Although recent studies show that tACS at higher frequencies, $140 \mathrm{~Hz}$ to $5 \mathrm{kHz}$ 
(Chaieb et al., 2010, Moliadze et al., 2010a) generates stronger, more robust aftereffects in the M1, its mechanism of action is yet to be determined. However, according to a study by Castro- Alamancos et al (Castro-Alamancos et al., 2007), frequencies of $10 \mathrm{~Hz}$ are generated by an excitatory network of interconnected pyramidal cells that are predominant due to the absence of GABAergic transmission. The transient facilitation in learning during the serial reaction time task (SRTT) reported by Antal et al (Antal et al., 2008) after application of $10 \mathrm{~Hz}$ tACS may be attributed to this mechanism. Aftereffects of tACS are thought to be caused by modulations in voltage- gated ion channels, more specifically the continual activation of rectified potassium channel currents and the potentiation of sodium channels (Francis et al., 2003).

\subsubsection{Transcranial Random Noise Stimulation (tRNS)}

It has already been shown that weak electric fields can effectively modulate neuronal activity (Jefferys, 1995, Nitsche and Paulus, 2000). We have recently demonstrated that the application of a randomly oscillating current applied over the M1 for 10mins at an intensity of $1 \mathrm{~mA}$, can induce neuroplastic aftereffects lasting up to 90 minutes poststimulation (Terney et al., 2008). tRNS aftereffects were also modulated by the execution of a mental task and the contraction of a target muscle; task performance during stimulation reduced TMS- evoked MEP amplitudes significantly compared to a passive condition where the subject is at rest. tRNS application also significantly improved performance, manifested as a reduction in reaction time, during the rendering of a cognitive task, a variant of the serial reaction time task (SRTT) used to quantify measures of implicit motor learning (Nissen and Bullmer, 1987). A similar finding was reported for studies using tDCS, where task performance during stimulation reversed tDCS induced aftereffects (Antal et al., 2007). A short duration application of tRNS (4 minutes) was shown to attenuate the BOLD response after performance of a simple finger- tapping task measured using functional magnetic resonance imaging (fMRI) (Chaieb et al., 2009). tRNS is a novel addition to the arsenal of transcranial electrical stimulation methods and so its mode of action is not yet fully characterized. Pairedpulse TMS measure of excitability reveal that short intracortical inhibition (SICI), long 
intracortical inhibition (LICI) and cortical silent period (cSP) are all unaffected by tRNS. However, MEPs measured using a paradigm measuring intracortical facilitation (ICF) showed an increase in amplitude poststimulation. This may suggest that tRNS aftereffects may be modulated via glutamatergic synapses (Ziemann et al., 1998a). Other potential mechanisms of action include the repeated activation of sodium channels via rectification due to the exposure of neuronal populations to a blanket field of high frequency stimulation (Bromm, 1968); depolarisation of the neuronal membrane causes an influx of sodium ions through voltage- gated sodium channels which when done repeatedly causes a heightened state of depolarisation in which sodium channels close and are then reopened by succeeding depolarisations. The net effect is an excitatory or artificially elevated response which is spread over the neural network. Studies on organotypic rat neuron cell cultures show that application of repetitive high frequency stimulation activated an inward sodium current which induced a weak depolarisation of the cell membrane (Schoen and Fromherz, 2008). The global effect of tRNS on the cerebral cortex has been observed, but is yet to be understood. There is evidence to suggest that neurons, when exposed to weak electrical fields, are capable of synchronizing (Francis et al., 2003). Studies done in vitro may give us useful insight into how tRNS effects can be propagated in the human cerebral cortex. In addition, tRNS is subject to the constraints of the classical stimulation montage with respect to electrode distance and placement (Moliadze et al., 2010b), but is not affected by the polarity of the positioning of the anodal or cathodal electrodes. During stimulation, tRNS is also virtually imperceptible and so is a useful tool in studies using patient populations (Ambrus et al., 2010).

\subsection{Factors influencing mechanisms of neuroplastic induction: Brain derived neurotrophic factor (BDNF) and measures of cortical excitability}

Brain- derived neurotrophic factor (BDNF) has many roles in the mammalian brain including cell proliferation and NMDA-receptor mediated neuroplasticity, and has been demonstrated in animal models (Figurov et al., 1996, Lu et al., 2005). Recent studies 
suggest that the expression of the BDNF gene may influence mechanisms of synaptic plasticity in both animal and human cortex and that this influences the efficacy of applied external electric and magnetic stimulation techniques (Cheeran et al., 2008, Fritsch et al., 2010, Yoshii and Constantine-Paton, 2010). The impact of BDNF on the induction and augmentation of neuroplastic effects in the mammalian cortex underlines the importance of optimizing non-invasive stimulation techniques; a common single nucleotide polymorphism (BDNF Val66Met), found in the normal population, was shown to impact upon the induction of neuroplastic aftereffects in a variety of protocols including tDCS and theta burst stimulation (TBS) paradigms. It was observed that the response of Met allele carriers differed significantly in these protocols compared with the response of Val66Val individuals. It is also of interest that both the neuroplastic aftereffects of tDCS and TBS are NMDA-receptor mediated (Liebetanz et al., 2002, Huang et al., 2007). BDNF may play a key role in how neuronal circuits undergo neuroplastic processes (Cheeran et al., 2008). These initial findings prompted us to retrospectively analyze our own data to explore whether this polymorphism is also important for other neuroplasticity-inducing tools. 


\subsection{Aims}

The aims of my thesis are to assay and characterise two novel methods of transcranial electric stimulation with the view to optimizing stimulation parameters to use within a clinical setting. As I have outlined a number of factors can influence how the human cortex responds to electrical current, whether they be oscillatory or constant, and I hope to establish a role for tRNS and tACS as robust and reliable methods of inducing neuroplastic effects quickly and safely within the human cortex on both neurophysiological and behavioural levels. The insight that we are able to gain from utilizing such techniques may allow for the application of transcranial electric stimulation techniques as useful tools in the elucidation of basic mechanisms of neuroplastic effects. 


\section{Chapter 2: Original Articles in the Thesis}

\subsection{Increasing human brain excitability by transcranial high-frequency random noise stimulation}

Terney D, Chaieb L, Moliadze V, Antal A, Paulus W. J Neurosci. 2008; 28(52):1414755.

The study concept was devised by Prof. W. Paulus and Prof. A. Antal; L. Chaieb and D. Terney collected the experimental data, and contributed significantly to the statistical analysis of the data. The article was written by D. Terney and L. Chaieb under the supervision of Prof. A. Antal and Prof. W. Paulus.

2.2 Short-duration transcranial random noise stimulation induces blood oxygenation level dependent response attenuation in the human motor cortex

Chaieb L, Kovacs G, Cziraki C, Greenlee M, Paulus W, Antal A. Exp Brain Res. 2009; 198(4):439-44.

The study concept was devised by Prof. A. Antal; L. Chaieb contributed significantly by collecting the experimental data and aiding in the data analysis. The manuscript was written by L. Chaieb with contributions from all authors.

2.3 Evaluating Aftereffects of Transcranial Random Noise Stimulation on Cortical Excitability

Chaieb L, Antal A, Paulus W. Under submission in Neural Plasticity.

The study concept was devised by Prof. W. Paulus, Prof. A. Antal and L. Chaieb; L. Chaieb contributed by designing the experimental protocol, collecting and analyzing the experimental data and writing the manuscript with contributions from Prof. Antal and Prof. Paulus. 
2.4 Transcranial alternating current stimulation in the low $\mathrm{kHz}$ range increases motor cortex excitability

Chaieb L, Antal A, Paulus W. Restorative Neurology and Neuroscience; In press.

The study concept was devised by Prof. W. Paulus and Prof. A. Antal; L. Chaieb contributed by designing the experimental protocol, collecting and analyzing the experimental data and writing the manuscript with contributions from Prof. A. Antal and Prof. W. Paulus.

2.5 Comparatively weak after-effects of transcranial alternating current stimulation (tACS) on cortical excitability in humans

Antal, A., Boros, K., Poreisz, C., Chaieb, L., Terney, D. \& Paulus, W. Brain Stimulation 2008; 1:97-105.

The study concept was devised by Prof. W. Paulus and Prof. A. Antal; L. Chaieb contributed by aiding in the collection of the experimental data, the analysis of the data and the writing of the manuscript.

2.6 Brain-derived neurotrophic factor (BDNF) gene polymorphisms shape cortical plasticity in humans

Antal A, Chaieb L, Moliadze V, Monte- Silva K, Poreisz C, Thirugnanasambandam N, Nitsche M, Shoukier M, Ludwig H, Paulus W. Brain Stimulation 2010.

The study concept was devised by Prof. A. Antal and Prof. W. Paulus; L. Chaieb contributed by aiding in the collection the experimental data and the analysis of the MEP-data. 


\section{Original Articles Connected to the Thesis}

\subsection{Frequency-dependent electrical stimulation of the visual cortex}

Kanai R, Chaieb L, Antal A, Walsh V, Paulus W. Curr Biol. 2008; 18(23):1839-43.

The study concept was devised by Prof. W. Paulus and Dr. Ryota Kanai; L. Chaieb contributed by teaching the methodological approach, collecting part of the experimental data, and aiding in the analysis of the data. The article was written by $\mathrm{R}$. Kanai with contributions from all authors.

2.8 Pergolide increases the efficacy of cathodal direct current stimulation to reduce the amplitude of laser-evoked potentials in humans

Terney D, Bergmann I, Poreisz C, Chaieb L, Boros K, Nitsche MA, Paulus W, Antal A. J Pain Symptom Manage. 2008; 36(1):79-91.

The study concept was devised by Prof. A. Antal and Prof. W. Paulus; L. Chaieb contributed by aiding in the collection of the experimental data.

2.9 Gender-specific modulation of short-term neuroplasticity in the visual cortex induced by transcranial direct current stimulation

Chaieb L, Antal A, Paulus W. Vis Neurosci. 2008; 25(1):77-81.

The study was devised by Prof. A. Antal based on data previously collected by the group of Prof. Antal; L. Chaieb performed the analysis of the data and wrote the manuscript under the supervision of Prof. A. Antal and Prof. W. Paulus. 


\subsection{Increasing human brain excitability by transcranial high- frequency random noise stimulation}

Terney D, Chaieb L, Moliadze V, Antal A, Paulus W. J Neurosci. 2008; 28(52):1414755.

Transcranial random noise stimulation (tRNS) is a novel addition to the burgeoning array of transcranial electrical stimulation techniques. Applied over the primary motor cortex (M1), the high-frequency randomly oscillating current induces long-lasting neuroplastic changes in the human cortex outlasting the duration of stimulation. When applied for 10 minutes, excitatory aftereffects, measured using monophasic singlepulse transcranial magnetic stimulation (TMS) - elicited motor- evoked potentials (MEPs), last up to 1 hour poststimulation. tRNS initially possesses a broad spectrum for stimulation, ranging between $0-640 \mathrm{~Hz}$. Our investigation focused on the partition of the stimulation spectrum into low $(0-100 \mathrm{~Hz})$ and high- frequency $(100-640 \mathrm{~Hz})$ components in order to examine the oscillating frequency responsible for the pronounced excitatory aftereffects. TMS elicited- MEP data acquisition showed that the high- frequency division of full spectrum tRNS was most effective in inducing neuroplastic aftereffects. tRNS also modified motor learning during the performance of a variant of the serial reaction time task (SRTT) and its aftereffects were altered by the performance of a cognitive and physical task during stimulation when compared to a passive condition. tRNS mode of action is yet to be fully elucidated, but excitatory effects are thought to arise for modulation of glutamatergic synapses as revealed by paired- pulse TMS measures investigating intracortical facilitation (ICF). Other putative mechanisms of action include the continued re- opening and voltage- gated sodium channels via rectification and the resultant repeated depolarisation of the neuronal membrane. 


\title{
Increasing Human Brain Excitability by Transcranial High- Frequency Random Noise Stimulation
}

\author{
Daniella Terney, Leila Chaieb, Vera Moliadze, Andrea Antal, and Walter Paulus \\ Department of Clinical Neurophysiology, Georg-August University, 37075 Göttingen, Germany
}

For $>20$ years, noninvasive transcranial stimulation techniques like repetitive transcranial magnetic stimulation (rTMS) and direct current stimulation (tDCS) have been used to induce neuroplastic-like effects in the human cortex, leading to the activity-dependent modification of synaptic transmission. Here, we introduce a novel method of electrical stimulation: transcranial random noise stimulation (tRNS), whereby a random electrical oscillation spectrum is applied over the motor cortex. tRNS induces consistent excitability increases lasting $60 \mathrm{~min}$ after stimulation. These effects have been observed in 80 subjects through both physiological measures and behavioral tasks. Higher frequencies $(100-640 \mathrm{~Hz})$ appear to be responsible for generating this excitability increase, an effect that may be attributed to the repeated opening of $\mathrm{Na}^{+}$channels. In terms of efficacy tRNS appears to possess at least the same therapeutic potential as rTMS/tDCS in diseases such as depression, while furthermore avoiding the constraint of current flow direction sensitivity characteristic of tDCS.

Key words: transcranial random noise stimulation (tRNS); primary motor cortex (M1); transcranial magnetic stimulation (TMS); serial reaction time task (SRTT); human; neuromodulation

\section{Introduction}

Neuroplasticity is an ongoing, self-organizing, adaptive process widespread in cortical areas; it allows the brain to learn and adapt to new environmental situations. External influences on neuroplastic processes may be used for functional improvement of diseases, in particular for improving cortical functions such as learning. The most well known method currently used to influence excitability of the brain by external means is transcranial magnetic stimulation (TMS) (Barker et al., 1985). It was followed by various repetitive stimulation paradigms, most recently by theta burst stimulation (TBS) (Huang et al., 2005). Although TBS increased the efficacy of rTMS by reducing stimulus intensity and the number of pulses required to achieve similar aftereffects, its upper safety limits are still unclear due to the potential risk of rTMS inducing seizures (Wassermann, 1998).

Another approach, weak transcranial direct current stimulation (tDCS) of the brain has so far avoided this risk. tDCS was investigated intermittently within the last four decades, but entered into neurobiological and clinical plasticity research only after its efficacy for modulating neuroplasticity could be unambiguously quantified by comparing TMS-induced motor-evoked potentials (MEPs) before and after tDCS (Nitsche and Paulus,

Received Sept. 4, 2008; revised 0ct. 16, 2008; accepted Nov. 12, 2008.

This work was initiated and funded by an unrestricted grant given by the Rose Foundation to develop new tools for the treatment of multiple sclerosis patients (D.T., L.C., W.P.) and the Bernstein Center for Computational Neuroscience Göttingen (V.M., A.A., W.P.) (BMBF 01GQ0432). We thank neuroConn for their cooperation and rapid adaptation of the stimulation device to our needs, and Michael Nitsche, Marom Bikson, and Klaus Schellhorn for their helpful comments.

The authors declare no competing financial interests.

Correspondence should be addressed to Andrea Antal, Department of Clinical Neurophysiology, Georg-August University, Robert-Koch-Strasse 40, 37075 Göttingen, Germany. E-mail: aantal@gwdg.de.

DOI:10.1523/JNEUROSCI.4248-08.2008

Copyright $\odot 2008$ Society for Neuroscience ～0270-6474/08/2814147-09\$15.00/0
2000, 2001). When compared with pulsed rTMS, tDCS represents the other end of the stimulation spectrum by delivering continuous electric current, which leads to "brain polarization." tDCS is able to induce long-lasting changes in cortical excitability in a reversible, relatively selective, painless, and safe manner. Generally, motor cortex (M1) excitability is enhanced by anodal and decreased by cathodal stimulation (Nitsche and Paulus, 2000).

Transcranial random noise stimulation (tRNS) of the human brain is a new technique (Fig. 1). Only one study so far has used noisy galvanic stimulation at a very low-frequency $(<2 \mathrm{~Hz})$ range targeting the vestibular nerves of patients with levodoparesponsive and unresponsive parkinsonism over a $24 \mathrm{~h}$ period (Yamamoto et al., 2005) and successfully improving parkinsonian symptoms. In this article, we demonstrate a new method of enhancing corticospinal excitability as measured by TMS, by applying weak tRNS for 10 min over the M1. Furthermore, a behavioral task was used to study tRNS-driven changes in performance during a variant of the serial reaction time task (SRTT) (Nissen and Bullemer, 1987), which is a standard paradigm to test implicit motor learning. In addition, we show how a cognitive or motor activity performed during stimulation can reduce the efficacy of tRNS, as previously described in studies using tDCS (Antal et al., 2007). The repeated potentiation of sodium channels has been suggested to be a putative mechanism of tRNS action; its aftereffects may outlast those observed after tDCS stimulation.

\section{Materials and Methods \\ Subjects}

Altogether, 80 healthy volunteers ( 32 men and 48 women; mean age, $25.74 \pm 5.13$ years; age range, $20-44$ years) were informed about all aspects of the experiments, and all gave informed consent. None of the subjects suffered from any neurological or psychological disorders, had metallic implants/implanted electric devices, or took any medication regularly, and none of them took any medication in the 2 weeks before their 

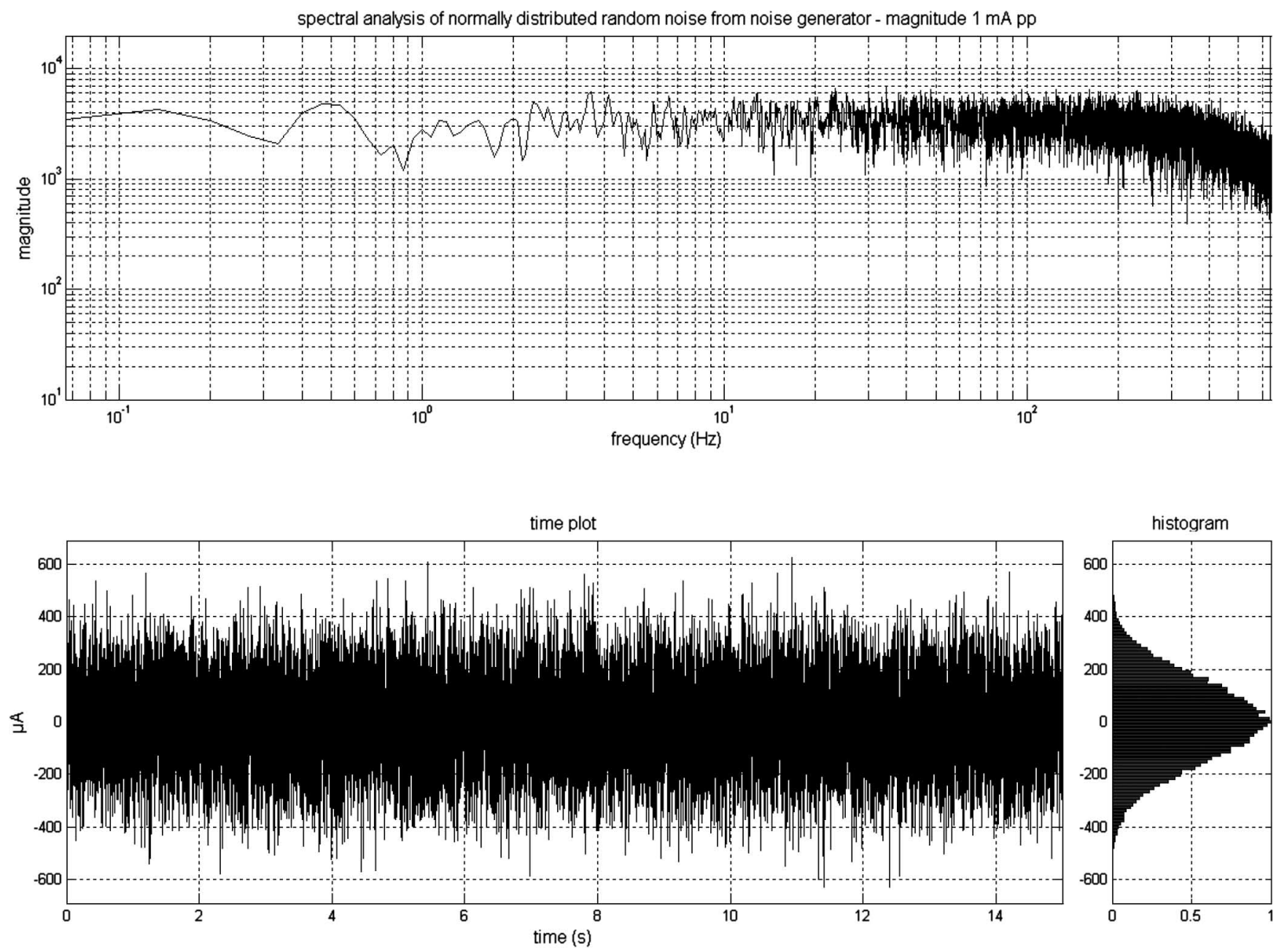

Figure 1. The output signal of DC-Stimulator PLUS, as a frequency distribution of the signal, the time plot of the signal, and a histogram. The signal was generated by a computer. In the stimulation mode "noise," there is a random level of current generated for every sample (sampling rate 1280 samples/s). The random numbers are normally distributed; the probability density function follows a bell-shaped curve. The amplitude of $1 \mathrm{~mA} \mathrm{pp}$ means that $99 \%$ of all generated amplitude values were between $+500 \mu \mathrm{A}$ and $-500 \mu \mathrm{A}$.

participation in any of the experiments. All subjects were right handed, according to the Edinburgh handedness inventory (Oldfield, 1971). We conformed to the Declaration of Helsinki, and the experimental protocol was approved by the Ethics Committee of the University of Göttingen.

Altogether, 47 healthy subjects (motor cortex: 17 participants; 21-27 years old; mean age $=23.71 \pm 2.08 ; 6$ male; low-frequency/high-frequency: 12 participants; $20-28$ years old; mean age $=23.83 \pm 3.28 ; 7$ male; DC-shift-induced excitability changes: 8 participants; $22-38$ years old; mean age $=25 \pm 5.12$; 4 male; premotor cortex: 10 subjects; $22-39$ years old; mean age $=26.5 \pm 6.31 ; 4$ male) participated in the singlepulse TMS study. Ten healthy subjects $(22-44$ years old; mean age $=$ $27.6 \pm 6.67 ; 3$ male) were involved in the paired-pulse TMS experiments, and four subjects participated in both single- and paired-pulse MEP experiments. Seventeen volunteers (22-31 years old; mean age $=25.29 \pm$ $2.89 ; 8$ male) took part in the implicit learning study. Twelve subjects were involved in the task-related modulation study (22-44 years old; mean age $=26.75 \pm 6.08 ; 4$ male). Three subjects participated both in the single-pulse MEP and in the implicit learning experiment. Two subjects were involved in both the single-pulse MEP and task-related modulation experiment.

\section{Random noise stimulation}

Stimulation was delivered by a battery-driven electrical stimulator (Version eldith DC-Stimulator-Plus, neuroConn) through conductiverubber electrodes, placed in two saline-soaked sponges. In the stimulation mode "noise" there is a random level of current generated for every sample (sampling rate $1280 \mathrm{samples/s).} \mathrm{The} \mathrm{random} \mathrm{numbers} \mathrm{are} \mathrm{nor-}$ mally distributed; the probability density function follows a bell-shaped curve. In the frequency spectrum all coefficients have a similar size ("white noise"). The noise signal contains all frequencies up to half of the sampling rate, i.e., a maximum of $640 \mathrm{~Hz}$ (Fig. 1). In a second experiment this frequency spectrum was separated into a low $(0.1-100 \mathrm{~Hz})$ - and high (101-640 Hz)-frequency spectrum. Because of the statistical characteristics, the signal has no DC offset, provided that the offset is set to zero.

The stimulation electrode was placed over the left motor cortex, which was determined by single pulse TMS. During the premotor single-pulse TMS study, the stimulation electrode was placed over the premotor cortex $(2.5 \mathrm{~cm}$ anterior from the motor cortex). To identify the primary motor and premotor cortex the same method was used as that implemented in previous TMS and tDCS studies (e.g., Fink et al., 1997; Münchau et al., 2002). The reference electrode was placed over the contralateral orbit. The size of the stimulation electrode was $4 \times 4 \mathrm{~cm}$ and the reference electrode was $6 \times 14 \mathrm{~cm}$. The electrodes were fixed by elastic bands. tRNS was applied for 10 min with a current strength of $1000 \mu \mathrm{A}$. The maximal current density was $62.5 \mu \mathrm{A} / \mathrm{cm}^{2}$ over the motor cortex, which is below the safety parameters accepted for tDCS (Nitsche et al., 2003). The current density was $11.9 \mu \mathrm{A} / \mathrm{cm}^{2}$ at the reference electrode. For sham stimulation the current was applied for $30 \mathrm{~s}$ at the beginning of the stimulation session, and then turned down. However, the screen on the stimulator did show the remaining time until the end of the stimulation session, as per the verum stimulation condition. Subjects were blinded for stimulation conditions in all of the studies. 


\section{Electrophysiological studies: transcranial magnetic stimulation}

To detect current-driven changes of excitability, motor-evoked potentials (MEPs) of the right first dorsal interosseus muscle (FDI) were recorded following stimulation of its motor-cortical representation field by single-pulse TMS. These were induced using a Magstim 200 magnetic stimulator, with a figure-of-eight standard double magnetic coil (diameter of one winding, $70 \mathrm{~mm}$; peak magnetic field, $2.2 \mathrm{~T}$; average inductance, $16.35 \mu \mathrm{H})$. The coil was connected to two monophasic Magstim 200 stimulators via a bistim module during the paired-pulse TMS study. Surface electromyogram (EMG) was recorded from the right FDI through a pair of $\mathrm{Ag}-\mathrm{AgCl}$ surface electrodes in a belly-tendon montage. Raw signals were amplified, bandpass filtered (2 $\mathrm{Hz}$ to $3 \mathrm{kHz}$; sampling rate, $5 \mathrm{kHz}$ ), digitized with a micro $1401 \mathrm{AD}$ converter (Cambridge Electronic Design) controlled by Signal Software (Cambridge Electronic Design, version 2.13), and stored on a personal computer for off-line analysis. Complete relaxation was controlled through auditory and visual feedback of EMG activity and whenever it was necessary, the subject was instructed to relax. The coil was held tangentially to the skull, with the handle pointing backwards and laterally at $45^{\circ}$ from the midline, resulting in a posterior-anterior direction of current flow in the brain. This orientation of the induced electrical field is thought to be optimal for predominantly transsynaptic mode of activation of corticospinal system. The optimum position was defined as the site where TMS resulted consistently in the largest MEP in the resting muscle. The site was marked with a skin marker to ensure that the coil was held in the correct position throughout the experiment.

\section{Experimental design}

Subjects were seated in a comfortable reclining chair with a mounted headrest throughout the experiments. Within each type of experimental session, the measurements were always performed by the same investigator.

\section{Single-pulse TMS}

Motor cortex stimulation. Seventeen subjects participated in two experimental sessions, on separate days, at least $3 \mathrm{~d}$ apart to avoid carryover effects. The subjects received $\mathrm{RN}$ and sham stimulation in a randomized order. Resting motor threshold (RMT), active motor threshold (AMT), the intensity to evoke MEP of $\sim 1 \mathrm{mV}$ peak-to-peak amplitude (SI1mV), and a baseline of TMS-evoked MEPs (40 stimuli) were recorded at 0.25 $\mathrm{Hz}$ before the stimulation.

Stimulus intensities (in percentage of maximal stimulator output) of TMS were determined at the beginning of each experiment. RMT was defined as the minimal output of the stimulator that induced a reliable MEP $(\sim 50 \mu \mathrm{V}$ in amplitude) in at least three of six consecutive trials when the FDI muscle was completely relaxed. AMT was defined as the lowest stimulus intensity at which three of six consecutive stimuli elicited reliable $\mathrm{MEP}(\sim 200 \mu \mathrm{V}$ in amplitude) in the tonically contracting FDI muscle (Rothwell et al., 1999).

Following stimulation, 40 single test-pulse MEPs were recorded at 0.25 $\mathrm{Hz}$, i.e., $\sim 0,5$, and $10 \mathrm{~min}$ after stimulation and then every $10 \mathrm{~min}$ up to 60 min.

Additionally, eight subjects underwent the same single-pulse TMS experiment (as described previously) to investigate the length of the aftereffect of the stimulation. Subjects were measured 0, 5, and $10 \mathrm{~min}$ after stimulation, then every $10 \mathrm{~min}$ up to $60 \mathrm{~min}$, then twice in the second hour, then 4,6 , and $24 \mathrm{~h}$ after stimulation. Both active and sham stimulation conditions were applied.

In a second sham-controlled experiment, the random noise frequency was divided into a low $(0.1-100 \mathrm{~Hz})$ - and high $(101-640 \mathrm{~Hz})$-frequency spectrum. Twelve participants underwent the same protocol as previously described.

To conclusively exclude DC-shift-induced excitability changes, eight subjects underwent the same protocol as previously described, in which the standard DC electrode montage was used (active electrode, anodal; reference electrode, cathodal) and then the electrode montage was reversed (cathodal-anodal).

Premotor cortex stimulation. Ten subjects participated in two experi- mental sessions on separate days, at least $3 \mathrm{~d}$ apart to avoid carryover effects. The subjects received tRNS and sham stimulation in a randomized order. The study protocol was performed as previously described.

\section{Paired-pulse TMS}

TMS measurements included RMT, AMT, and SI $1 \mathrm{mV}$, short-interval intracortical inhibition (SICI)/intracortical facilitation (ICF), longinterval intracortical inhibition (LICI), recruitment curves, and cortical silent period (CSP).

Ten subjects participated in four experimental sessions [(1) tRNS: recruitment curves and SICI/ICF; (2) tRNS: LICI and CSP; (3) sham: recruitment curves and SICI/ICF; and (4) sham: LICI and CSP] on separate days at least $3 \mathrm{~d}$ apart to avoid carryover effects. The subjects received RN and sham stimulation in a randomized order. Stimulus intensities (in percentage of maximal stimulator output) of TMS were determined at the beginning of each experiment. SI $1 \mathrm{mV}$ was determined with single-pulse TMS first (the amplitude of the test MEP was matched before and after tRNS). RMT and AMT were defined as previously mentioned.

SICI/ICF and LICI were measured with two different protocols of single- and paired-pulse TMS applied in a random order at $0.25 \mathrm{~Hz}$. For SICI/ICF, two magnetic stimuli were given through the same stimulating coil, and the effect of the first (conditioning) stimulus on the second (test) stimulus was investigated (Kujirai et al., 1993). To avoid any floor or ceiling effect, the intensity of the conditioning stimulus was set to a relatively low value of $80 \%$ of AMT. The test-stimulus intensity was adjusted to SI $1 \mathrm{mV}$. SICI was measured with interstimulus intervals (ISI) of 2 and $4 \mathrm{~ms}$, and ICF with ISIs of 9, 12, 15, and $25 \mathrm{~ms}$. The control condition (test pulse alone) was tested 40 times, and each of the conditioning-test stimuli 20 times. The mean peak-to-peak amplitude of the conditioned MEP at each ISI was expressed as a percentage of the mean peak-to-peak size of the unconditioned test pulse. The second protocol tested LICI with two suprathreshold stimuli applied with ISIs of 50, 100, 150, and $200 \mathrm{~ms}$ (Valls-Solé et al., 1992). The intensity of both stimuli was set to $110 \%$ of RMT. Here as well, the intensity was set to this relatively low value to avoid any floor or ceiling effect. The control condition (first pulse alone) was tested 40 times, whereas each of the paired stimuli was tested 20 times. LICI was taken as the mean percentage inhibition of conditioned MEP at ISIs of 50, 100, 150, and $200 \mathrm{~ms}$.

Recruitment curves were measured with three different and increasing stimulus intensities $(110 \%, 130 \%$, and $150 \%$ of RMT), each with 10 pulses. A mean was calculated for all intensities. Finally, 10 pulses with SI $1 \mathrm{mV}$ and 10 pulses with $120 \%$ RMT were applied under tonic contraction of the right first dorsal interosseus muscle. CSPs were separately determined, in rectified and averaged EMG traces with a prestimulus period of $100 \mathrm{~ms}$. CSP (in ms) was measured from the TMS stimulus to the point where the signal reached the amplitude of the mean prestimulus EMG activity again for $>5 \mathrm{~ms}$.

\section{Behaviorial studies}

\section{SRTT}

Subjects were seated in front of a computer screen at eye level behind a response pad with four buttons numbered 1-4 and were instructed to push each button with a different finger of the right hand (index finger for button 1, middle finger for button 2, ring finger for button 3, and little finger for button 4). An asterisk appeared in one of four positions that were horizontally spaced on a computer screen and permanently marked by dots. The subjects were instructed to press the key corresponding to the position of the asterisk as fast as possible. After a button was pushed, the go signal disappeared. The next go signal was displayed $500 \mathrm{~ms}$ later. The test consisted of eight blocks of 120 trials. In blocks 1 and 6, the sequence of asterisks followed a pseudorandom order in that asterisks were presented equally frequently in each position and never in the same position in two subsequent trials. In blocks $2-5,7$, and 8 , the same 12 trial sequence of asterisk positions repeated itself 10 times (abadbcdacbdc). Subjects were not informed about the repeating sequence.

In six subjects, the first three blocks of the previously used test were repeated 1 (block 9: pseudorandom; blocks 10-11: repeated sequences) and 2 h (block 12: pseudorandom; blocks 13-14: repeated sequences) 
after stimulation. Differences in performance between blocks 9-10 and 12-13 also represent a measure of implicit learning. In the SRTT study, the current was delivered during the blocks $2-5$, which lasted $\sim 7 \mathrm{~min}$. The order of verum and sham stimulation was randomized. The current was always ramped up or down over the first and last $2 \mathrm{~s}$ of stimulation.

\section{Task-related modulation of $t R N S$}

The three experimental sessions were conducted in a repeatedmeasurement design using a randomized order, with a break of at least $3 \mathrm{~d}$ between each session. First, the left motor-cortical representational field of the right FDI was identified using TMS. After determining the resting and active motor thresholds, a baseline of TMS-evoked MEPs (25 stimuli) was recorded at $0.25 \mathrm{~Hz}$. Afterward, one stimulation electrode was fixed at the representational field of the right FDI, and the other was fixed at the contralateral forehead above the orbita.

During tRNS, subjects were passively sitting during the stimulation (experiment 1), had their attention directed toward a cognitive test (experiment 2) or were instructed to push a ball in their right hand (experiment 3). After termination of RNS, 25 MEPs were recorded every fifth minute up to $30 \mathrm{~min}$ and then every $15 \mathrm{~min}$ up to $2 \mathrm{~h}$.

During the stimulation in experiment 2, the subjects were required to fill out a cognitive test that was presented on a computer monitor. The subjects had to push a suitable button with their right index finger to give the correct answer. The test was presented in German and downloaded from a commercial intelligence test homepage. The questions were on a variety of subjects. In experiment 3 , the subjects were instructed to push a ball $(8 \mathrm{~cm}$ diameter) in their right hand. The ball was connected to a display where the actual values related to pressure were quantified. Before the stimulation session, the subjects were asked to push the ball as hard as possible. During the tRNS session, subjects had to push the ball to half-maximal contraction as previously shown.

\section{Safety}

\section{Neuron-specific enolase determination}

To assess the safety of tRNS, we measured serum neuron-specific enolase (NSE), a sensitive marker of neuronal damage, evident in many neurological disorders, e.g., in epilepsy (Steinhoff et al., 1999). Elevated NSE concentration is a specific marker in intractable temporal lobe epilepsy. A blood sample for NSE-measurement was taken in six healthy subjects before and $10 \mathrm{~min}$ after stimulation. Furthermore, in one subject, who was stimulated for 8 consecutive days, this measurement was done on every day.

\section{EEG recording}

The EEG was recorded using a three-channel montage. One electrode was placed over $\mathrm{Oz}$ and two laterally above the motor region (C3 and C4) in accordance with the international 10/20 system. The impedance was kept at $<5 \mathrm{k} \Omega$. Linked mastoids (RLm) were used as a reference. The ground electrode was positioned on the forehead. Data were collected with a sampling rate of $1000 \mathrm{~Hz}$ using BrainAmp system (Brain Products) and were analyzed off-line (Brain Vision Analyzer, Brain Products).

The EEG experiments were conducted in a repeated-measurement design (tRNS and sham) using a randomized order, with a minimum break of $1 \mathrm{~d}$ between each stimulation session. Two minutes EEG was recorded at rest before and three times after stimulation (immediately and 7 and $14 \mathrm{~min}$ after the end of the stimulation). EEG epochs (2 min) were segmented for $30 \mathrm{~s}$ and filtered by using $0.1 \mathrm{~Hz}$ ( $24 \mathrm{~dB} /$ octave) low cutoff and a $70 \mathrm{~Hz}$ ( $24 \mathrm{~dB} /$ octave) high cutoff and $50 \mathrm{~Hz}$ notch filters. In addition to semiautomatic artifact detection (200 $\mu \mathrm{V}$ amplitude criterion), all epochs were visually inspected, and those containing eye blinks or muscle movement artifacts were excluded. After artifact rejection, all of the epochs were segmented into $2 \mathrm{~s}$, and fast Fourier transformation (FFT) was calculated for all electrodes $(0.5 \mathrm{~Hz}$ resolution, and $10 \% \mathrm{Ham}$ ming window). The FFT segments were averaged for each $30 \mathrm{~s}$. The mean activity in voltage was calculated and exported from each frequency bands (theta band $4.5-7 \mathrm{~Hz}$, alpha band $8-12 \mathrm{~Hz}$, beta band $12.5-30 \mathrm{~Hz}$, and gamma band $31-49 \mathrm{~Hz}$ ) for statistical analysis.

For sham stimulation, the current was turned on for $30 \mathrm{~s}$ at the begin- ning of the stimulation. Subjects were blinded for stimulation conditions in all of the studies.

\section{Data analyses}

\section{Electrophysiological studies}

Single-pulse TMS. Repeated measurements of ANOVAs [condition $($ tRNS vs sham) $\times$ time (before; $0,5,10,20,30,40,50,60$ min after stimulation; ( $n=8$ : before; $0,5,10,20,30,40,50,60,90 \mathrm{~min}$ and 2, 4, 6, $24 \mathrm{~h}$ after stimulation)] were used to compare the different conditions. Effects were considered significant if $p<0.05$. In the case of a significant interaction of time and stimulation condition, a Tukey's post hoc test was performed. Student's $t$ test was used to compare the motor thresholds (RMT, AMT, and SI $1 \mathrm{mV}$ ) between experimental sessions. All data are given as means \pm SEM.

Paired-pulse TMS. For each measurement (SI1mV, RMT, AMT, SICI, ICF, LICI, and CSP), we performed separate ANOVAs for repeated measurements by using the mean values from each subject as the dependent variable. In addition to the factor "stimulation type" (tRNS vs sham), the ANOVA model included the factor "ISI" (2, 4, 7, 9, 12, 15, and $25 \mathrm{~ms}$ ) when SICI and ICF was analyzed, the factor "intensity" (100\%, 130\%, and $150 \%$ of RMT) for recruitment curves, or the factor "intensity" $(120 \%$ RMT and SI $1 \mathrm{mV}$ ) for CSP. A $p$ value of $<0.05$ was considered significant for all statistical analyses. In the case of a significant interaction between ISI/intensity and stimulation condition, a Tukey post hoc test was performed. Student's $t$ test was used to compare the motor thresholds (RMT, AMT, and SI $1 \mathrm{mV}$ ) between experimental sessions. Data are expressed as mean \pm SEM.

\section{Behavioral studies}

SRTT analysis. Concerning the implicit learning paradigm, statistical analysis was performed with repetitive-measures ANOVA (independent variables current condition and block) for reaction time (RT), error rate (ER), and variability. As the RT and ER differences between blocks 5 and 6 are thought to represent an exclusive measure of implicit learning, interactive Student's $t$ tests were performed to compare the respective differences between tRNS and sham conditions. In each trial, RT was measured from the appearance of the "go" signal until the first button was pushed by the subject. For each block of trials of a given experimental condition, mean RT was calculated for each subject separately. Furthermore, as a measure of the variability of the RTs, we have calculated the coefficient of variation (the ratio of the SD to the mean $\times 100$ ). An ER was calculated to assess the number of incorrect responses for each block and each subject in each stimulation condition.

Task-related modulation of $t R N S$. Repeated-measures ANOVA [experiment (passive vs cognitive/motor) $\times$ time (before and 5, 10, 15, 20, 25, and $30 \mathrm{~min}$ after stimulation, then every $15 \mathrm{~min}$ up to $2 \mathrm{~h}$ )] was used to compare different task conditions during tRNS. Effects were considered significant if $p<0.05$. In case of the significant interaction of time and stimulation condition, a Tukey post hoc test was performed. Student's $t$ test was used to compare the motor thresholds (RMT, AMT, and SI $1 \mathrm{mV}$ ) between experimental sessions. All data are given as means + SEM.

\section{Safety}

NSE determination. Two-tailed $t$ tests (paired samples, critical $p$ value 0.05 ) were performed to compare NSE values before and after tRNS.

EEG recording. To compare the effect of stimulation on the EEG spectrum, a repeated-measures ANOVA (independent variable: tRNS vs sham $\times$ time points after stimulation; dependent variable: FFT power in a given frequency band) was calculated.

\section{Results}

All of the subjects tolerated the stimulation; none of the experimental sessions were interrupted due to side effects of the stimulation. Only two of 80 subjects reported a slight burning sensation under the electrodes during the stimulation. 
Table 1. Results of the statistical analyses in the case of the single- and paired-pulse TMS studies over the primary motor cortex

\begin{tabular}{|c|c|c|c|c|c|}
\hline & Measurement & Factor & $\mathrm{df}$ & $F / t^{a}$ & $p$ \\
\hline \multicolumn{6}{|l|}{ Single-pulse TMS } \\
\hline \multirow[t]{3}{*}{ Student's $t$ test } & RMT & & 10 & 0.90 & 0.39 \\
\hline & AMT & & 10 & 1.68 & 0.12 \\
\hline & SI1mV & & 10 & 0.42 & 0.69 \\
\hline \multirow[t]{3}{*}{ ANOVA } & & Condition & 1 & 7.24 & 0.01 \\
\hline & & Time & 28 & 4.01 & $<0.01$ \\
\hline & & Condition $\times$ time & 28 & 3.53 & $<0.01$ \\
\hline \multicolumn{6}{|l|}{ Paired-pulse TMS } \\
\hline \multirow[t]{3}{*}{ Student's $t$ test } & RMT & & 9 & 0.42 & 0.68 \\
\hline & AMT & & 9 & 0.90 & 0.39 \\
\hline & SI1mV & & 9 & 0.01 & 1.00 \\
\hline \multirow[t]{15}{*}{ ANOVA } & & Condition & 1 & 0.80 & 0.39 \\
\hline & & Intensity & 2 & 19.03 & $<0.01$ \\
\hline & RECR & Condition $\times$ intensity & 2 & 0.38 & 0.69 \\
\hline & & Condition & 1 & 0.38 & 0.54 \\
\hline & & ISI & 1 & 47.94 & $<0.01$ \\
\hline & $\mathrm{SICl}$ & Condition $\times|S|$ & 1 & 0.13 & 0.73 \\
\hline & & Condition & 1 & 0.58 & 0.46 \\
\hline & & $|S|$ & 3 & 0.88 & 0.46 \\
\hline & ICF & Condition $\times|S|$ & 3 & 5.56 & $<0.01$ \\
\hline & & Condition & 1 & 0.23 & 0.64 \\
\hline & & $|S|$ & 4 & 4.04 & 0.01 \\
\hline & LICI & Condition $\times|S|$ & 4 & 0.37 & 0.83 \\
\hline & & Condition & 1 & 0.63 & 0.44 \\
\hline & & Intensity & 1 & 1.05 & 0.33 \\
\hline & CSP & Condition $\times$ intensity & 1 & 0.81 & 0.38 \\
\hline
\end{tabular}

RECR, Recruitment curves. Bold indicates significant values. ${ }^{a}$ for ANOVA and $t$ for Student's $t$ test.

\section{Electrophysiological studies: MEPs}

Single-pulse TMS

When 10 min tRNS was applied over the primary motor cortex, the induced excitability increases rose up to $20-50 \%$, as revealed by TMS. They last for $60 \mathrm{~min}$ after stimulation. Repeated measurements of ANOVA revealed a significant main effect of condition $\left(F_{(1,28)}=7.24, p=0.01\right)$ and time $\left(F_{(8,224)}=4.01, p<\right.$ $0.001)$ in the case of motor cortex stimulation. The interaction between condition and time was also significant $\left(F_{(8,224)}=3.53\right.$, $p<0.001$ ) (Table 1). According to the post hoc analysis, significantly increased MEPs were observed at the 5 and 10-60 min time points compared with the time point before $(p<0.05)$ tRNS (Fig. 2).

RMT, AMT, and SI1mV baseline values were compared between RN and sham stimulation conditions using Student's $t$ test. There was no significant difference between tRNS and sham stimulation in any of the measurements (Table 1).

Furthermore, we have separated the stimulation spectrum into low (0.1-100 Hz)- and high (101-640 Hz)-frequency ranges. High-frequency stimulation was more effective with regard to changing the level of cortical excitability. Repeated measurements of ANOVA revealed a significant effect of condition $\left(F_{(1,21)}=4.2, p=0.05\right)$ when the high-frequency spectrum stimulation was used, compared with the sham condition. However, there was no significant effect of condition, when the lowfrequency spectrum was applied $\left(F_{(1,20)}=2.22, p=0.15\right)$. There was no significant condition $\times$ time interaction $\left(F_{(7,147)}=1.62\right.$, $p=0.13$ and $F_{(7,140)}=0.78, p=0.61$, respectively) (Fig. 3).

We did not observe any changes in corticospinal excitability when the premotor cortex was stimulated, implying that the effect of tRNS over the M1 is indeed focal. Repeated measurements of ANOVA revealed no significant effect on condition $\left(F_{(1,18)}=\right.$ $0.01, p=0.99)$ nor time $\left(F_{(8,14)}=0.78, p=0.61\right)$. There was no significant condition $\times$ time interaction $\left(F_{(8,14)}=0.69, p=0.70\right)$.

The possibility of a hidden DC shift in the stimulation spectrum as a cause of the excitability increase was excluded by the results of a control experiment conducted by reversing the connection of the electrodes to the stimulator. In the case of measuring DC-shift-induced excitability changes, repeated measurements of ANOVA revealed no significant effect of condition $\left(F_{(1,14)}=0.29, p=0.60\right)$. The effect of time was significant $\left(F_{(8,112)}=\right.$ $2.13, p=0.04)$. There was no significant condition $\times$ time interaction $\left(F_{(8,112)}=\right.$ $0.24, p=0.98$ ).

\section{Paired-pulse TMS}

In our paired-pulse TMS study, we have observed an increase in ICF after tRNS over M1. Repeated measurements of ANOVA revealed no significant effect of condition $\left(F_{(1,9)}=0.58, p=0.46\right)$ or ISI $\left(F_{(3,27)}=0.88, p=0.46\right)$. However, the interaction between condition and ISI was significant $\left(F_{(3,27)}=5.56, p=0.004\right)$. According to the post hoc analysis, significantly increased MEPs were observed at ICF of 12 and $15 \mathrm{~ms}$ after tRNS compared with the sham condition $(p<0.05)$.

tRNS administration had no effect on SICI, LICI, CSP, or motor-evoked recruitment curves as revealed by repeated measurements of ANOVA (Table 1).

\section{Behavioral studies}

SRTT

With regard to the functional effect of tRNS, it significantly improved performance in the acquisition and early consolidation phase of motor learning. This was primarily represented by the differences between blocks 5 and 6 between tRNS and sham conditions, which are exclusive measurements of implicit learning. Compared with the sham stimulation condition, RTs in the SRTT shortened during tRNS of the primary motor cortex; and subjects became faster during the course of the experiment.

Repeated-measures ANOVA revealed a significant effect on blocks $\left(F_{(7,112)}=37.59, p<0.001\right)$. This was caused by an interaction of tRNS versus sham stimulation for block 5 and block 6 , due to a greater difference in the case of $\operatorname{tRNS}(t=-2.87, \mathrm{df}=16$, $p=0.01)$ as revealed by Student's $t$ tests. There was no significant effect on stimulation. However, the stimulation $\times$ blocks interaction was marginally significant $\left(F_{(7,112)}=1.95, p=0.06\right)$. Figure 4 shows the differences between RN and sham stimulation. The paradigm was repeated in six subjects after 1 and $2 \mathrm{~h}$ after stimulation. At these time points the RTs were not significantly different between the tRNS and sham stimulation conditions (see Fig. 4). However, the RTs of the sham and tRNS trials were not the same as those observed after the familiar blocks immediately after stimulation, but are the same after $1 \mathrm{~h}$; the control RTs decreased substantially in the $1 \mathrm{~h}$ period for the familiar block, which may represent consolidation of learning, whereas this was not the case for the tRNS group. Nevertheless, only six subjects were analyzed after $1 \mathrm{~h}$.

For the ER, the ANOVAs showed a significant main effect on 
blocks $\left(F_{(7,112)}=2.54, p=0.02\right)$. Despite this, the results of all other tests remained insignificant. Student's $t$ tests revealed no significant difference between blocks 5 and 6. For RT variability, the ANOVAs showed a significant main effect on blocks $\left(F_{(7,112)}\right.$ $=29,12, p<0.0001)$ without significant interaction between condition and blocks.

\section{Task-related modulation of $t R N S$}

Excitability increase induced by tRNS was modified by paying attention to a task involving mental activity and by contraction of the target muscle during the stimulation. Following tRNS, the amplitude of the MEPs was increased in the passive condition, slightly decreased in the cognitive condition and markedly reduced in the motor condition. When the amplitude of the MEPs was compared with regard to the passive condition and cognitive task before and after stimulation, repeatedmeasures ANOVA revealed a main effect of experiment $\left(F_{(1,11)}=5.45, p=0.04\right)$, but time $\left(F_{(12,132)}=0.50, p=0.91\right)$ was not significant. The interaction between the experiment and time was significant $\left(F_{(12,132)}=2.36, p=0.009\right)$. The post hoc test revealed that, after $t R N S$ in the passive condition, significantly increased MEP amplitudes were observed up to $20 \mathrm{~min}$, and at the 1 and $2 \mathrm{~h}$ time points when compared with the cognitive task condition $(p<0.01)$. When the amplitude of the MEPs was compared with the passive condition and motor task, repeated measures of ANOVA revealed a main effect of experiment $\left(F_{(1,11)}=10.05, p=0.009\right)$, but time $\left(F_{(12,132)}=0.74, p=0.71\right)$ was not significant. The interaction between the experiment and time was significant $\left(F_{(12,132)}=3.96, p<0.001\right)$. The post hoc test revealed that, after tRNS in the passive condition, significantly increased MEP amplitudes were observed up to $25 \mathrm{~min}$ ( $p<$ 0.01 ) compared with the motor condition.

\section{Safety}

The concentration of serum NSE was unchanged after tRNS. Student's $t$ test showed no significant difference between the before and after stimulation NSE concentrations of six healthy subjects $(t=$ $0.09, p=0.93$, mean value before stimulation: $6.96 \pm 1.84 \mu \mathrm{g} / \mathrm{L}$, after stimulation: $6.91 \pm 1.7 \mu \mathrm{g} / \mathrm{L})$. One subject was stimulated for $10 \mathrm{~min}$ every day for 8 consecutive days. The NSE values did not change significantly over the period from the first to last day of stimulation $(t=-0.2, p=$ 0.87 , mean value before stimulation: $9.57 \pm 2.2 \mu \mathrm{g} / \mathrm{L}$, after stimulation: $9.53 \pm 3.0 \mu \mathrm{g} / \mathrm{L})$.

Furthermore, we recorded EEGs before and after tRNS and at baseline.

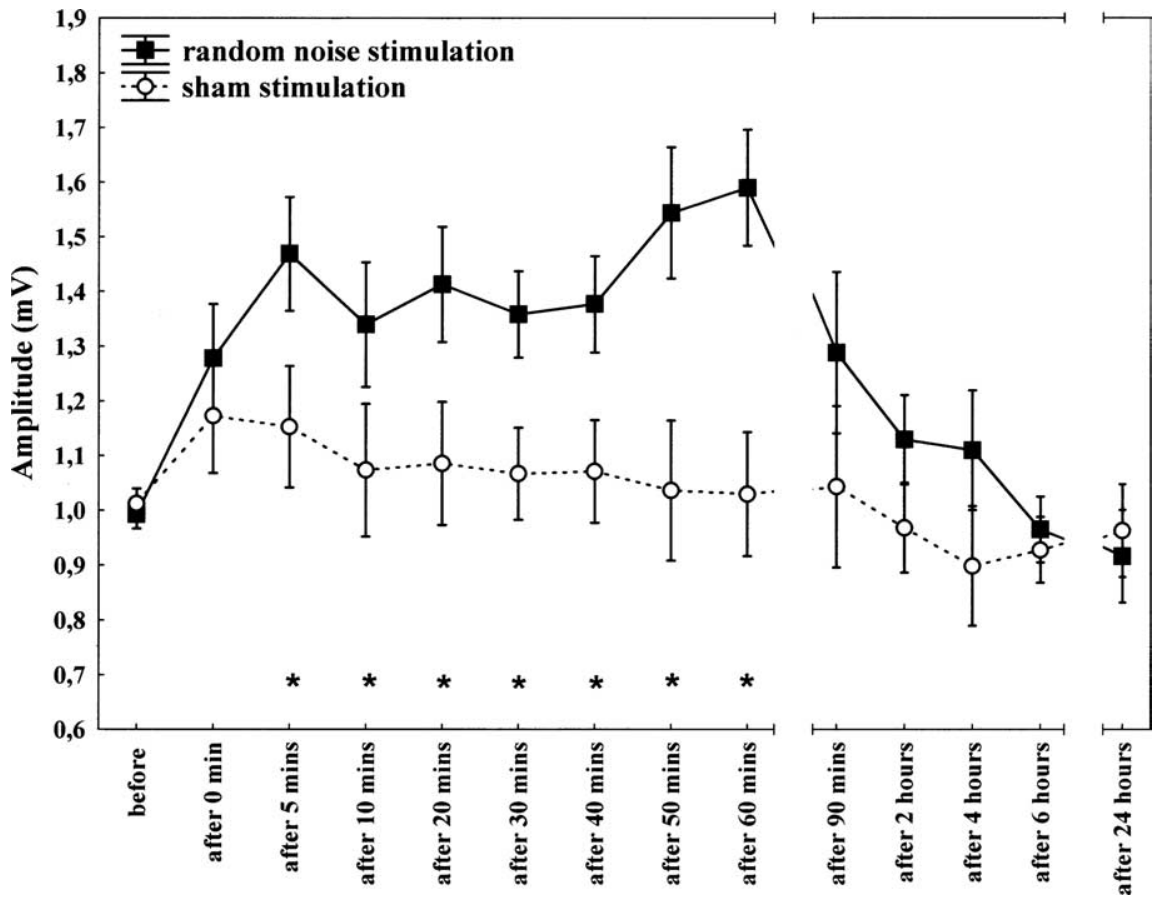

Figure 2. Effect of $10 \mathrm{~min}$ RN stimulation on motor-evoked potentials. Time course of motor cortex excitability changes lasting for 60 min after stimulation, shown after $10 \mathrm{~min}$ RN stimulation over M1 at $1 \mathrm{~mA}$ compared with sham stimulation. The figure shows mean amplitudes and their SEMs up to $60 \mathrm{~min}$ (including all subjects, $n=17$ ) and between $90 \mathrm{~min}$ and $24 \mathrm{~h}$ (including 8 subjects). Asterisks indicate significant differences between MEP amplitudes after 5 and $10-60$ min after stimulation and those

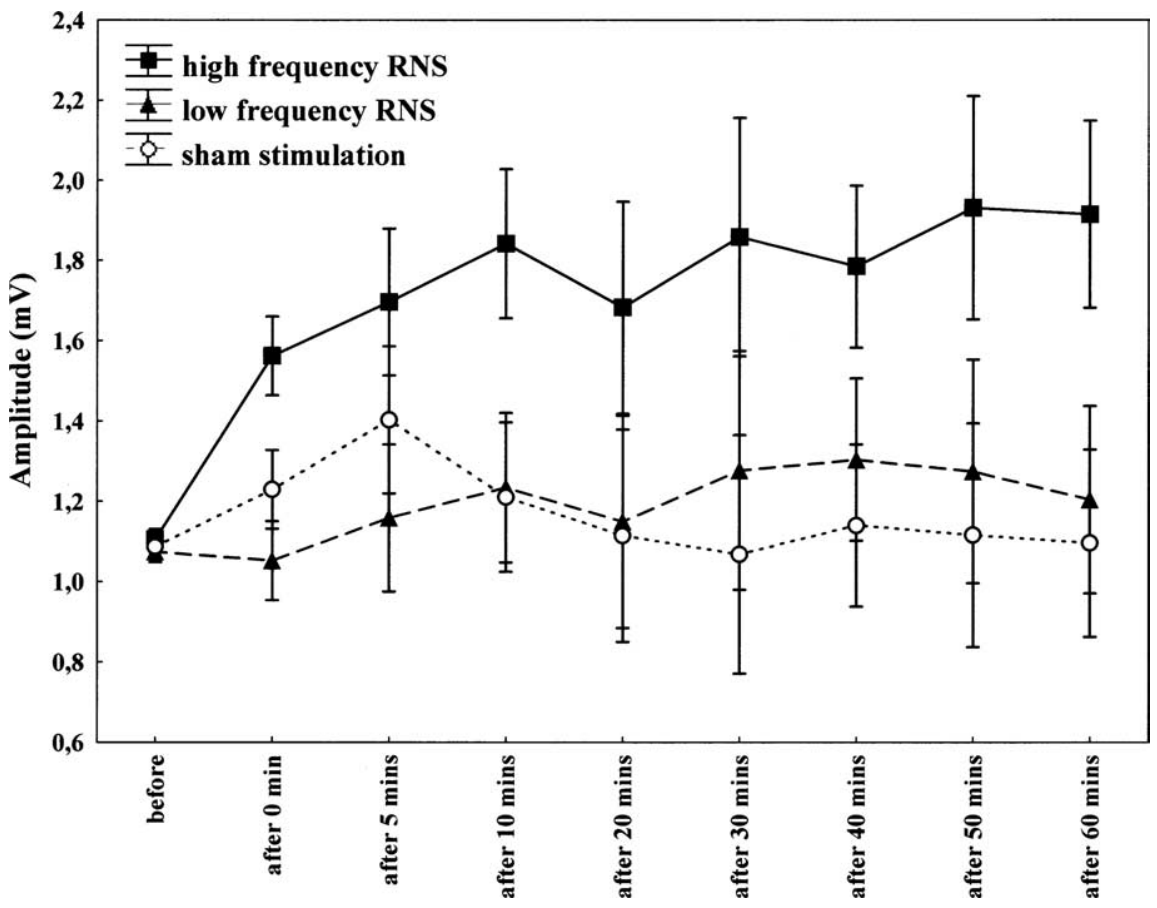

Figure 3. Effect of 10 min of low $(0.1-100 \mathrm{~Hz})$ - and high $(101-640 \mathrm{~Hz})$-frequency RN stimulation on motor-evoked potentials. Time course of motor cortex excitability changes lasting for $60 \mathrm{~min}$ after stimulation, shown after 10 min of high-frequency RN stimulation over M1 at $1 \mathrm{~mA}$ compared with low-frequency and sham stimulation. The figure shows mean amplitudes and their SEMs up to $60 \mathrm{~min}$ (including all subjects, $n=12$ ).

did not find any significant difference regarding any of the frequency bands. Repeated-measures ANOVA revealed no significant interactions between current conditions, time, or channels for any of the different frequencies applied (see supplemental 

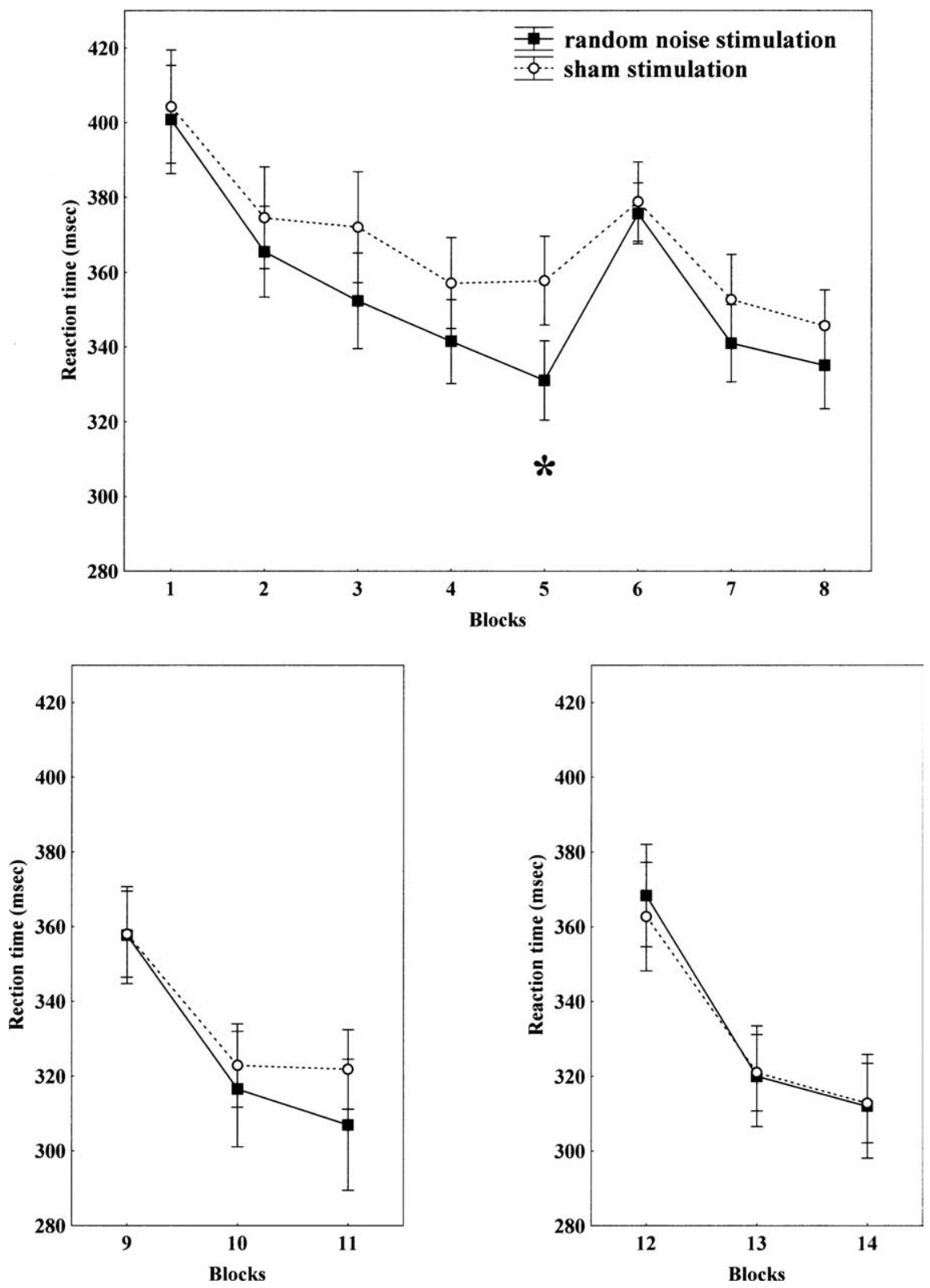

Figure 4. tRNS of the primary motor cortex improves implicit motor learning in its early phase. Reaction times decrease faster in the tRNS condition when compared with the sham stimulation condition (top). Moreover, the RT difference comparing blocks 5 and 6, which indicates implicit sequence learning, is bigger for the tRNS condition, when compared with sham condition. The asterisk indicates a significant difference regarding reaction time differences between blocks 5 and 6 between RN and sham stimulation. In 1 and $2 \mathrm{~h}$ after stimulation, this significant difference was no longer detectable (bottom panels).

Table 2, available at www.jneurosci.org as supplemental material). Additionally, we did not see any abnormal EEG activity after tRNS. Therefore, we can conclude that limited exposure to tRNS of the cortex using the parameters we applied here is safe.

\section{Discussion}

In this study, we demonstrate that weak tRNS over M1 enhances corticospinal excitability both during and after stimulation in the healthy human brain. Furthermore, our results suggest that the high-frequency subdivision of the whole tRNS spectrum between 100 and $640 \mathrm{~Hz}$ is functionally responsible for inducing excitability in the M1. In terms of commonly used noninvasive excitability parameters, we found an increased ICF after tRNS over M1 using the paired-pulse paradigm. tRNS application had no effect on
SICI, LICI, CSP, or motor-evoked recruitment curves [for an overview of methods used to study the modulation of human motor cortex excitability in local circuits, see Paulus et al. (2008) and Ziemann et al. (2008)]. Pharmacological studies show that among others, ICF is most likely to be mediated by the glutamatergic system ( $\mathrm{Zi}$ emann et al., 1998), possibly by the activation of glutamatergic synapses by tRNS. However, no clear evidence was found concerning the cortical origin of ICF, in a recent study in which epidural recording was applied in a conscious subject (Di Lazzaro et al., 2006). The results of this study showed that, despite a significant increase in MEP at ISIs of 10 and $15 \mathrm{~ms}$, there is no evident change in the descending volley. Thus at ISIs of 10 and $15 \mathrm{~ms}$, a small conditioning stimulus can produce clear facilitation of MEPs even though it leads to no detectable change in descending corticospinal activity.

The average MEP decrease observed after mental effort and motor activation are in agreement with previous studies using tDCS (Antal et al., 2007) and paired associative stimulation (PAS) (Stefan et al., 2004). Similarly, a recent study observed that contraction of the FDI muscle during TBS abolished the effects of stimulation on the MEPs (Huang et al., 2008). These results suggest that externally induced neuronal plasticity is highly dependent on the state of the subject during stimulation.

It appears that the tRNS-driven cortical excitability change facilitates the learning process. Previous studies suggest that an excitability enhancement coincides with facilitating the learning process by inducing the strengthening of synapses and inducing long-term potentiation via modifying NMDA-receptor efficacy (RioultPedotti et al., 2000). Regarding studies in the human, this is in line with previous observations of increased activation of the M1 during motor learning tasks (Grafton et al., 1992; Honda et al., 1998), showing that effects of motor training can be improved by cortical excitability enhancements. Additionally, our results describing an increase in corticospinal excitability and facilitating learning with regard to the SRTT more closely resemble those reported by previous studies after anodal tDCS (Nitsche and Paulus, 2000, 2001); even more so, since we applied well proven tDCS parameters such as electrode position, intensity, and stimulation duration.

There is, however, a key difference between tDCS and tRNS. tDCS modifies the transmembrane neuronal potential directly and thus modulates the firing rate of individual neurons (Bindman et al., 1964). In contrast, the stimulation spectrum of tRNS does not possess a DC component. In addition, the physiological control experiment conducted by reversing the electrode position did not influence the characteristic excitability-enhancing 
aftereffect, in contrast to the inhibition that we see with cathodal tDCS (Nitsche and Paulus, 2000). Several physiological mechanisms may underlie the tRNS effects. tRNS, like alternating current stimulation (tACS) (Antal et al., 2008), can possibly interfere with ongoing oscillations and neuronal activity in the brain and thus result in a cortical excitability increase. However, tACS with intensities of $>400 \mu \mathrm{A}$ (Antal et al., 2008) induced a flickering sensation via retinal stimulation, and as a result [at least in the frequency range that we applied $(1-45 \mathrm{~Hz})$ ], we were reluctant to increase the intensity further, at least with the standard reference montage at the forehead close to the retina. Also, the tACS type of monophasic sinusoidal stimulation is more likely to be epileptogenic than that of a random noise waveform. For this reason, we started by using a random noise frequency spectrum with a range of $0.1-640 \mathrm{~Hz}$; the latter frequency is known to represent the high end of physiologically measured human electric brain oscillations (Gobbelé et al., 2000).

We did not make current density calculations of how effectively the high-frequency component of the stimulus is transmitted to the brain. There is, however, sufficient evidence to suggest that the current used here can reach the brain. The bone is the structure with highest resistance, and has to be considered primarily when stimulating the head electrically (Wagner et al., 2008). In fact, high bone resistance was the reason why TMS replaced pulsed electrical stimulation in 1985 (Barker et al., 1985) and thereby could avert painful stimulation. It was found that the bone conductivity on the three orthogonal directions was constant up to $10 \mathrm{kHz}$ (Reddy and Saha, 1984) even above the range of the frequencies used in our study. Distinctly higher frequencies of $50 \mathrm{kHz}$ could still pass through the skull as measured by electrical impedance tomography (Abascal et al., 2008). The dielectric properties of bone was shown to be constant between frequencies of 10 and 100,000 Hz (Gabriel et al., 1996).

A previous study by Yamamoto et al. (2005) used a distinctly lower frequency range $(<2 \mathrm{~Hz})$ in patients with Parkinson's disease (PD). Their method, however, differed from ours in electrode position, stimulation amplitude, duration, and techniques of evaluation. Improved autonomic and motor functions were detected after $24 \mathrm{~h}$ of continuous noisy vestibular electrical stimulation over the bilateral mastoids. The authors hypothesized that in PD patients the input noise ameliorated the impaired neuronal transmission, and the noise itself enhanced weak neuronal signal detection in the sensory system; the phenomenon of stochastic resonance, as shown in several experimental studies (e.g., Moss et al., 2004). Indeed, it has been suggested that noisy electrical fluctuations can boost synaptic signals.

Stochastic resonance may play a role in tRNS, however at much higher frequency ranges. For some years now, oscillations within a frequency range of $80-200 \mathrm{~Hz}$ (ripples) have been associated with plasticity processes (Grenier et al., 2001) and learning (Ponomarenko et al., 2008). Another putative mechanism of tRNS may be activation of sodium channels via rectification by high-frequency stimulation (Bromm, 1968). The postulated tRNS effect begins with the depolarization of a neuronal membrane which causes $\mathrm{Na}^{+}$channels to open. This allows an influx of $\mathrm{Na}^{+}$ions to flow down the concentration gradient and increases membrane depolarization. If the $\mathrm{Na}^{+}$entry is insufficient, there is no regenerative depolarization and thus no action potential, just the "local response." The repolarization occurs passively over a longer period of time compared with the duration of $\mathrm{Na}^{+}$ion entry. If stimulation is repeated, the $\mathrm{Na}^{+}$channels can reopen and induce a second $\mathrm{Na}^{+}$ion influx, which depolarizes the membrane further, heightening the effect of the preceding depolarization. The $\mathrm{Na}^{+}$channels then close, and after repolarization can be reopened by succeeding depolarizations. Indeed, recently it was shown that repetitive extracellular highfrequency stimulation in cultured rat neurons activated an inward sodium current, which gave rise to a weak depolarization of the cell membrane (Schoen and Fromherz, 2008). Although the time integral of the stimulating current used in a voltage clamp study was zero, the average membrane potential was shifted in the direction of depolarization. The resulting depolarization was understood to be the result of the nonlinearity of the sodium current-voltage input during subthreshold excitation. Since we used a symmetric high-frequency stimulation, this nonlinearity could be the reason for the excitatory effects we have seen with tRNS. Interestingly, the effect of tRNS increased with time after stimulation. Effects induced by "repetitive activation of $\mathrm{Na}^{+}$channels by weak capacitive currents" studied by Schoen and Fromherz (2008) also increase with stimulation time, however within a much shorter time range $(<1 \mathrm{~s})$. On the other hand, continuous opening of $\mathrm{Na}^{+}$channels would lead to membrane depolarization, from which we can assume from previous tDCS studies that a time range of $>3$ min may lead to LTP-like mechanisms. However, the neuronal membrane is a more intricate structure and possesses numerous voltage-gated ion channels and is subject to simultaneous influxes of ionic currents $\left(\mathrm{Ca}^{2+}, \mathrm{K}^{+}\right.$, etc). Indeed, because the membrane is encumbered with multiple voltagegated channels, that are "nonlinear," the aforementioned induced changes in membrane fluctuation can be amplified. In summary, a pure DC stimulus can open $\mathrm{Na}^{+}$channels just once, whereas repeated pulses (tRNS) can induce multiple ionic influxes, and achieve substantially heightened effects. The interval at which the pulses are repeated must be short and relates to the time constant of the nerve membrane.

Thus, finally, the neuroplastic effects of tRNS could be analogous to anodal tDCS aftereffects, but with clear advantages. tRNS can circumvent problems that can arise by stimulating a folded cortex with anodal stimulation, since on one side of the gyrus wall current orientation induces excitation, while on the opposite side of the gyrus, it will inevitably induce inhibition. When using tRNS only excitatory aftereffects are observable. Also "tangential" stimulation of nerve cells now appears to be possible with tRNS. Within a "tangential" DC electric field applied to a symmetrical dendritic arbor, currents on both sides would cancel each other at the axon hill. In the case of a rectifying depolarization using a fast oscillating field, the cell would be depolarized regardless of current flow orientation. Safety concerns are probably lessened than in the case of tDCS. Several anecdotal, but so-far-unpublished, reports have described small skin burns after tDCS. In general, nonpolarizing currents seem to be safer than polarizing currents as seen in deep brain stimulation. Here we have not observed any tRNS-induced changes with EEG recordings (see supplemental Table 2, available at www.jneurosci.org as supplemental material). tRNS using $1 \mathrm{~mA}$ was unnoticed in 78 of 80 subjects, compared with a slight skin tingling sensation with tDCS. Thus it appears to have the best blinding potential for controlled studies of currently available methods.

In summary, tRNS allows an unnoticeable and thus painless, selective, focal, noninvasive, and reversible excitability increase of the cortex. Apart from being more economically viable than rTMS its main advantage seems to be the direction insensitivity of the stimulation. It seems to provide a qualitatively new way of producing and interfering with brain plasticity. 


\section{References}

Abascal JF, Arridge SR, Atkinson D, Horesh R, Fabrizi L, De Lucia M, Horesh L, Bayford RH, Holder DS (2008) Use of anisotropic modelling in electrical impedance tomography; description of method and preliminary assessment of utility in imaging brain function in the adult human brain. Neuroimage 43:258-268.

Antal A, Terney D, Poreisz C, Paulus W (2007) Towards unravelling taskrelated modulations of neuroplastic changes induced in the human motor cortex. Eur J Neurosci 26:2687-2691.

Antal A, Boros K, Poreisz C, Chaieb L, Terney D, Paulus W (2008) Comparatively weak after-effects of transcranial alternating current stimulation (tACS) on cortical excitability in humans. Brain Stim 1:97-105.

Barker AT, Jalinous R, Freeston IL (1985) Non-invasive magnetic stimulation of human motor cortex. Lancet 1:1106-1107.

Bindman LJ, Lippold OCJ, Redfearn JWT (1964) The action of brief polarizing currents on the cerebral cortex of the rat (1) during current flow and (2) in the production of long-lasting after-effects. J Physiol 172:369-382.

Bromm B (1968) Die Natrium-Gleichrichtung der unterschwellig erregten Membran in der quantitative Formulierung der Ionentheorie. Pflugers Arch 302:233-244.

Di Lazzaro V, Pilato F, Oliviero A, Dileone M, Saturno E, Mazzone P, Insola A, Profice P, Ranieri F, Capone F, Tonali PA, Rothwell JC (2006) Origin of facilitation of motor-evoked potentials after paired magnetic stimulation: direct recording of epidural activity in conscious humans. J Neurophysiol 96:1765-1771.

Fink GR, Frackowiak RS, Pietrzyk U, Passingham RE (1997) Multiple nonprimary motor areas in the human cortex. J Neurophysiol 77:2164-2174.

Gabriel S, Lau RW, Gabriel C (1996) The dielectric properties of biological tissues: III. Parametric models for the dielectric spectrum of tissues. Phys Med Biol 41:2251-2269.

Gobbelé R, Waberski TD, Kuelkens S, Sturm W, Curio G, Buchner H (2000) Thalamic and cortical high-frequency $(600 \mathrm{~Hz})$ somatosensory-evoked potential (SEP) components are modulated by slight arousal changes in awake subjects. Exp. Brain Res 133:506-513.

Grafton ST, Mazziotta JC, Presty S, Friston KJ, Frackowiak RS, Phelps ME (1992) Functional anatomy of human procedural learning determined with regional cerebral blood flow and PET. J Neurosci 12:2542-2548.

Grenier F, Timofeev I, Steriade M (2001) Focal synchronization of ripples $(80-200 \mathrm{~Hz})$ in neocortex and their neuronal correlates. J Neurophysiol 86:1884-1898.

Honda M, Deiber MP, Ibáñez V, Pascual-Leone A, Zhuang P, Hallett M (1998) Dynamic cortical involvement in implicit and explicit motor sequence learning. A PET study. Brain 121:2159-2173.

Huang YZ, Edwards MJ, Rounis E, Bhatia KP, Rothwell JC (2005) Theta burst stimulation of the human motor cortex. Neuron 45:201-206.

Huang YZ, Rothwell JC, Edwards MJ, Chen RS (2008) Effect of physiological activity on an NMDA-dependent form of cortical plasticity in human. Cereb Cortex 18:563-570.

Kujirai T, Caramia MD, Rothwell JC, Day BL, Thompson PD, Ferbert A, Wroe S, Asselman P, Marsden CD (1993) Corticocortical inhibition in human motor cortex. J Physiol 471:501-519.

Moss F, Ward LM, Sannita WG (2004) Stochastic resonance and sensory information processing: a tutorial and review of application. Clin Neurophysiol 115:267-281.

Münchau A, Bloem BR, Irlbacher K, Trimble MR, Rothwell JC (2002) Functional connectivity of human premotor and motor cortex explored with repetitive transcranial magnetic stimulation. J Neurosci 22:554-561.
Nissen MJ, Bullemer P (1987) Attentional requirements of learning: evidence from performance measures. Cognit Psychol 19:1-32.

Nitsche MA, Paulus W (2000) Excitability changes induced in the human motor cortex by weak transcranial direct current stimulation. J Physiol 527:633-639.

Nitsche MA, Paulus W (2001) Sustained excitability elevations induced by transcranial DC motor cortex stimulation in humans. Neurology 57:1899-1901

Nitsche MA, Liebetanz D, Lang N, Antal A, Tergau F, Paulus W (2003) Safety criteria for transcranial direct current stimulation (tDCS) in humans. Clin Neurophysiol 114:2220-2222; author reply 2222-2223.

Oldfield RC (1971) The assessment and analysis of handedness: the Edinburgh inventory. Neuropsychologia 9:97-113.

Paulus W, Classen J, Cohen LG, Large CH, Di Lazzaro V, Nitsche MA, Pascual-Leone A, Rosenow F, Rothwell JC, Ziemann U (2008) State of the art: pharmacologic effects on cortical excitability measures tested by transcranial magnetic stimulation. Brain Stim 1:151-163.

Ponomarenko AA, Li JS, Korotkova TM, Huston JP, Haas HL (2008) Frequency of network synchronization in the hippocampus marks learning. Eur J Neurosci 27:3035-3042.

Reddy GN, Saha S (1984) Electrical and dielectrical properties of wet bone as a function of frequency. IEEE Trans Biomed Eng 31:296-303.

Rioult-Pedotti MS, Friedman D, Donoghue JP (2000) Learning-induced LTP in neocortex. Science 290:533-536.

Rothwell JC, Hallett M, Berardelli A, Eisen A, Rossini P, Paulus W (1999) Magnetic stimulation: motor evoked potentials: the International Federation of Clinical Neurophysiology. Electroencephalogr Clin Neurophysiol Suppl 52:97-103.

Schoen I, Fromherz P (2008) Extracellular stimulation of mammalian neurons through repetitive activation of $\mathrm{Na}^{+}$channels by weak capacitive currents on a silicon chip. J Neurophysiol 100:346-357.

Stefan K, Wycislo M, Classen J (2004) Modulation of associative human motor cortical plasticity by attention. J Neurophysiol 92:66-72.

Steinhoff BJ, Tumani H, Otto M, Mursch K, Wiltfang J, Herrendorf G, Bittermann HJ, Felgenhauer K, Paulus W, Markakis E (1999) Cisternal S100 protein and neuron-specific enolase are elevated and site-specific markers in intractable temporal lobe epilepsy. Epilepsy Res 36:75-82.

Valls-Solé J, Pascual-Leone A, Wassermann EM, Hallett M (1992) Human motor evoked responses to paired transcranial magnetic stimuli. Electroencephalogr Clin Neurophysiol 85:355-364.

Wagner T, Eden U, Fregni F, Valero-Cabre A, Ramos-Estebanez C, PronioStelluto V, Grodzinsky A, Zahn M, Pascual-Leone A (2008) Transcranial magnetic stimulation and brain atrophy: a computer-based human brain model study. Exp Brain Res 186:539-550.

Wassermann EM (1998) Risk and safety of repetitive transcranial magnetic stimulation: report and suggested guidelines from the International Workshop on the Safety of Repetitive Transcranial Magnetic Stimulation, June 5-7, 1996. Electroencephalogr Clin Neurophysiol 108:1-16.

Yamamoto Y, Struzik ZR, Soma R, Ohashi K, Kwak S (2005) Noisy vestibular stimulation improves autonomic and motor responsiveness in central neuro-degenerative disorders. Ann Neurol 58:175-181.

Ziemann U, Chen R, Cohen LG, Hallett M (1998) Dextromethorphan decreases the excitability of the human motor cortex. Neurology 51:1320-1324.

Ziemann U, Paulus W, Nitsche MA, Pascual-Leone A, Byblow WD, Berardelli A, Siebner HR, Classen J, Cohen LG, Rothwell JC (2008) Consensus: motor cortex plasticity protocols. Brain Stim 1:164-182. 


\subsection{Short-duration transcranial random noise stimulation induces blood oxygenation level dependent response attenuation in the human motor cortex}

Chaieb L, Kovacs G, Cziraki C, Greenlee M, Paulus W, Antal A. Exp Brain Res. 2009; 198(4):439-44.

Transcranial random noise stimulation (tRNS) has been shown to increase levels of cortical excitability for up to 1 hour poststimulation after a 10 minute application over the primary motor cortex (M1) (Terney et al., 2008). In this study we have chosen to investigate a shorter stimulation duration (4minutes at $1 \mathrm{~mA}$ ) in combination with the performance of a simple finger- tapping task using functional magnetic resonance imaging (fMRI). Results indicated that tRNS over the left hemispheric sensorimotor cortex resulted in a decrease in the blood oxygenated level dependent (BOLD) response in the hand area. This suggests that the performance of a task under the influence of stimulation may result in changeable aftereffects, and that the duration of stimulation may play a role in the observed aftereffects; longer stimulation durations for tRNS may result to a different neuroplastic- inducing mechanism than that of shorter durations. The study also illustrates that need to further optimize stimulation parameters to achieve potent aftereffects poststimulation. 


\title{
Short-duration transcranial random noise stimulation induces blood oxygenation level dependent response attenuation in the human motor cortex
}

\author{
Leila Chaieb · Gyula Kovacs · Csaba Cziraki • \\ Mark Greenlee $\cdot$ Walter Paulus $\cdot$ Andrea Antal
}

Received: 8 April 2009 / Accepted: 30 June 2009 / Published online: 2 August 2009

(C) Springer-Verlag 2009

\begin{abstract}
Manipulation of cortical excitability can be experimentally achieved by the application of transcranial random noise stimulation (tRNS). TRNS is a novel method of non-invasive electrical brain stimulation whereby a random electrical oscillation spectrum is applied over the cortex. A previous study recently reported that application of weak 10-min tRNS over primary motor cortex (M1) enhances corticospinal excitability both during and after stimulation in the healthy human brain. Here, blood oxygenation level dependent (BOLD) MRI was used to monitor modulations in human sensorimotor activity after the application of 4-min tRNS. Activation maps for a right hand index-thumb finger opposition task were obtained for nine subjects after sham and 1-mA tRNS in separate sessions. TRNS of the left-hemispheric sensorimotor cortex resulted in a decrease in the mean number of activated pixels by $17 \%$, in the hand area. Our results indicate that tRNS applied with different durations and/or in combination with a task might result in different outcomes. Application of tRNS to the human cortex allows an unnoticeable and thus painless, selective, non-invasive and reversible activity
\end{abstract}

L. Chaieb $(\varangle) \cdot$ W. Paulus · A. Antal

Department of Clinical Neurophysiology,

Georg-August University, Robert-Koch-Str. 40,

37075 Göttingen, Germany

e-mail: leilachaieb@med.uni-goettingen.de

G. Kovacs · C. Cziraki · M. Greenlee

Institute for Experimental Psychology,

University of Regensburg, Albert-Magnus-Str.,

93040 Regensburg, Germany

G. Kovacs

Department of Cognitive Science,

Budapest University of Technology and Economics,

1111 Budapest, Hungary change within the cortex, its main advantage being the direction insensitivity of the stimulation. TRNS also provides a qualitatively new way of producing and interfering with brain plasticity, although, further research is required to optimise stimulation parameters and efficacy.

Keywords Transcranial random noise stimulation . Motor cortex · fMRI finger tapping · Cortical inhibition

\section{Introduction}

Interacting with the neural activity of the primary motor cortex (M1) may allow us to investigate mechanisms of neuroplasticity in cortical networks and ultimately develop potential therapeutic approaches to neurological disorders with altered cortical excitability. In a previous study using motor-evoked potential (MEP) recordings and psychophysical measurements we have shown that a 10-min application of transcranial random noise stimulation (tRNS) can enhance cortical excitability for up to $90 \mathrm{~min}$ (Terney et al. 2008). TRNS is a novel technique, and an opportunity to extend the spectrum of already well-established external stimulation methods like transcranial direct current stimulation (tDCS) and repetitive transcranial magnetic stimulation (rTMS). Applied over the M1, it was shown to induce facilitation of MEPs, an effect outlasting the 10-min stimulation duration, with aftereffects lasting up to 90-min poststimulation (Terney et al. 2008). TRNS possesses many advantages over currently used techniques. For example, as it is an oscillatory current and therefore does not have the polarity constraints of tDCS, or the perceptible skin sensations, when being applied. Stimulation conditions can also be randomised, as it possesses no sensory or auditory cues when used, as opposed to the inability to administer rTMS 
in a 'sham' setting, which makes tRNS a good candidate for therapeutic intervention within a clinical setting (for a review see Fregni and Pascual-Leone 2007).

Earlier experiments investigating external electrical stimulation techniques have lead to tDCS becoming a wellestablished method of inducing enduring changes in corticospinal excitability. Research shows that anodal application of tDCS over the M1 causes an increase in cortical excitability, whilst cathodal tDCS has the reverse effect (Nitsche and Paulus 2000, 2001). In this study, we adopted the stimulation parameters of tDCS and applied them to stimulation protocols using tRNS. A previous study by Baudewig et al. (2001) demonstrated for the first time that blood oxygenation level dependent (BOLD) fMRI is capable of revealing transient changes in neuronal excitability, after a short-duration application of tDCS over the sensorimotor cortex. In this study, cathodal tDCS resulted in a $57 \%$ decrease of activated voxels in the supplementary motor area, but no change in the hand area of the M1. Here we aim to provide evidence that tRNS also has the ability to modulate levels of cortical excitability using a similar paradigm.

In this study, the applied stimulating currents were designed to have the 1/f-type power spectrum characteristic of noise measured in the nervous system. In another study, noisy vestibular galvanic stimulation also benefited daytime trunk activity dynamics and improved cognitive performance in patients with levodopa responsive Parkinson's disease and levodopa unresponsive Parkinsonism (Yamamoto et al. 2005). The rationale behind this method is the beneficial role played by input noise in sensitizing neuronal systems, making it possible to detect weak subthreshold signals. This technique can be used as a sensory prosthesis through a mechanism known as stochastic resonance (Wiesenfeld and Moss 1995). The fine tuning of noise in the nervous system may in turn lead to a change in the state of synchrony in oscillating neural networks, having farreaching effects on the mechanisms of central processing. In turn, this may provide an additional means of cortical stimulation, which is not subject to polarity and once optimised, may induce enduring therapeutically relevant aftereffects.

In this study, we aimed to observe and record the effects of weak tRNS on cortical excitability using fMRI and a simple finger-tapping technique.

\section{Materials and methods}

\section{Subjects}

Nine healthy subjects participated in the study (3 females, age range 21-32 years). All subjects gave their informed consent prior to participating in the experiments. The experiments were approved by the Ethics Committee of the University of Göttingen, and all experiments conformed to the Declaration of Helsinki. All subjects were right-handed according to the Edinburgh Handedness Inventory (Oldfield 1971). None of the subjects suffered from any neurological condition nor took any regular medication and had no metallic implants or pacemakers.

Transcranial random noise stimulation

Electrical stimulation was delivered by a battery-driven constant-current stimulator (Version DC-Stimulator-Plus, NeuroConn GmbH, Ilmenau, Germany) through conductive-rubber electrodes, encased in two saline-soaked sponges. In the 'noise' stimulation mode, a random level of current is generated for every sample (sampling rate 1,280 $\mathrm{sps)}$. The randomised frequency is normally distributed; the probability density function follows a bell-shaped curve. Within the frequency spectrum all coefficients have a similar size (white noise). The noise signal contains all frequencies up to half of the sampling rate, i.e. a maximum of $640 \mathrm{~Hz}$. Because of the statistical characteristics the signal has no DC offset, provided the offset is set to zero.

One electrode was placed over the left sensorimotor area (the middle of the electrode was at $\mathrm{C} 3$ according to the 10 20 EEG system). The other electrode was placed over the contralateral orbit. The size of the electrode over the sensorimotor cortex was $4 \times 4 \mathrm{~cm}$, and the electrode placed over contralateral orbit was $6 \times 14 \mathrm{~cm}$. The electrodes were fixed by elastic bands. tRNS was applied for 4 min with a current strength of $1 \mathrm{~mA}$. The maximal current density was $62.5 \mu \mathrm{A} / \mathrm{cm}^{2}$ over the sensorimotor cortex, which is below the safety parameters accepted for tDCS (Nitsche et al. 2003). The current density for the reference electrode was $12 \mu \mathrm{A} / \mathrm{cm}^{2}$. Subjects were blinded for stimulation conditions throughout the experiment.

\section{fMRI and finger-tapping task}

Imaging was performed using a 3-Tesla MR head scanner (Siemens Allegra, Erlangen, Germany). For the functional series we continuously acquired 285 images with 29 interleaved axial slices using a standard $\mathrm{T} 2 *$ weighted echo-planar imaging $(\mathrm{EPI})$ sequence $(\mathrm{TR}=1700 \mathrm{~ms}, \mathrm{TE}=30 \mathrm{~ms}$, flip angle $=90^{\circ}, 64 \times 64$ matrixes, in-plane resolution $3 \times 3 \mathrm{~mm}$, slice thickness $3 \mathrm{~mm}$ ). After the functional scans, high-resolution sagittal T1-weighted images were acquired using a magnetization prepared rapid gradient echo sequence (MP-RAGE; TR $=2250 \mathrm{~ms}$, TE $=2.6 \mathrm{~ms}$, $1-\mathrm{mm}$ isotropic voxel size) to obtain a 3D structural scan. The parameters of the MP-RAGE sequence were adapted from the Alzheimer's disease Neuroimaging Initiative project (ADNI; http://www.loni.ucla.edu/ADNI/). This sequence 

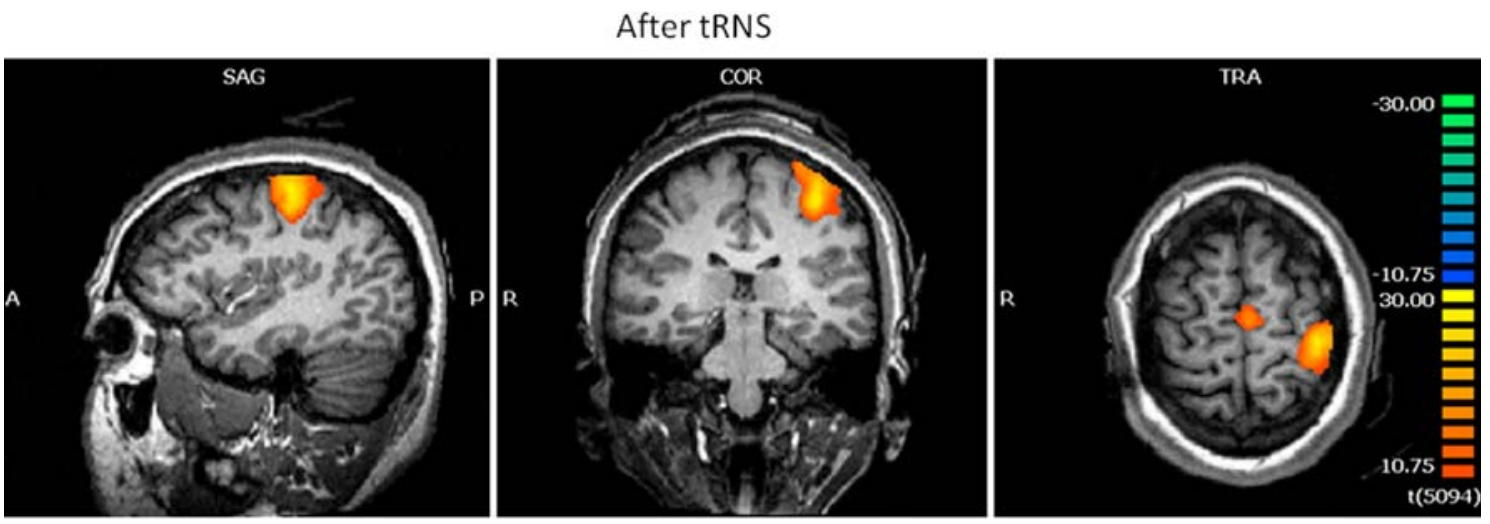

\section{After sham stimulation}
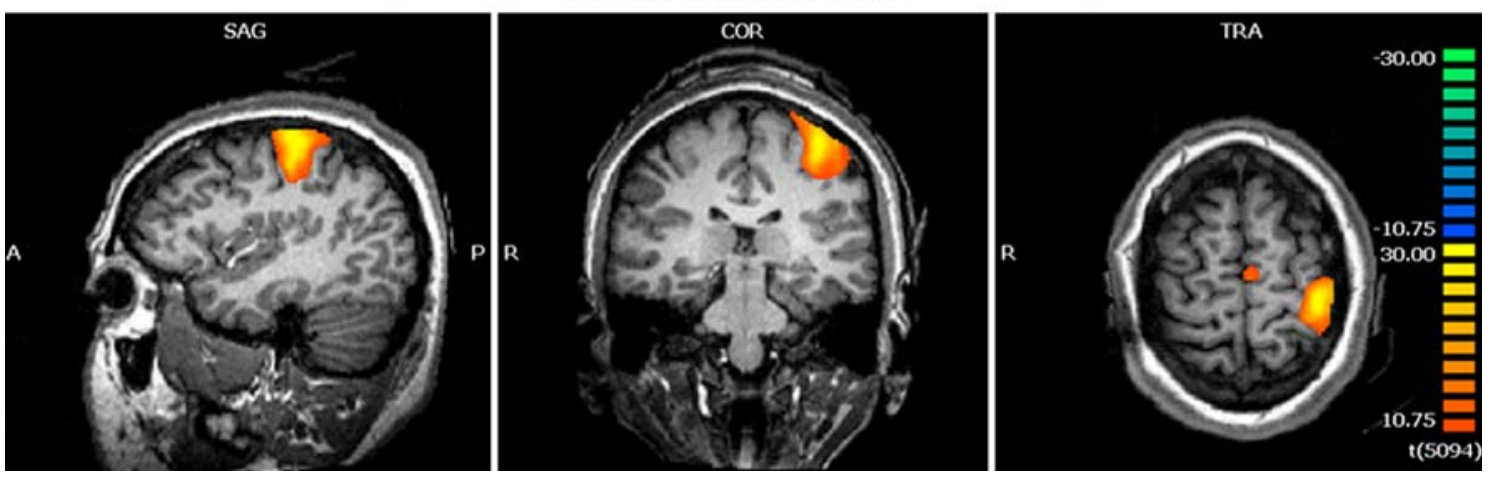

Fig. 1 Activation maps showing the main effects of the two different stimulation conditions for MOVEMENT $(n=9)$ pooled together for the individual subject groups. The activation maps were thresholded at $P<0.0001$ (Bonferroni-corrected). Please note the electrode position over the M1

has been optimized to differentiate between white and grey matter.

The tRNS montage was in place prior to each subject entering the scanner. The subject was then required to begin the prescribed finger-tapping task when a cue was displayed on the screen in front of the subject whilst inside the scanner. The subject was asked to tap the first forefinger and thumb of the right hand at $1 \mathrm{~Hz}$. The subject continued to finger tap until a stop cue was displayed. Prior to execution of the finger-tapping task, the subjects received tRNS for $4 \mathrm{~min}$ at $1 \mathrm{~mA}$, or sham stimulation in a randomised order. The subject was then placed in the scanner to begin the task. The sequence of stimulation (real versus sham) was counterbalanced between subjects. Subjects were required to perform the finger-tapping task twice, with a break of at least $40 \mathrm{~min}$ between the conditions. Subjects were unaware as to the stimulation condition being given.

Visual stimuli (green dot with regard to tapping, red dot with regard to resting) were presented with Presentation 9.9 (Neurobehavioral Systems Inc., Albany, Canada) on a standard PC and back projected via an LCD video projector (JVC, DLA-G20, Yokohama, Japan) onto a translucent circular screen (approximately $30^{\circ}$ diameter), placed inside the scanner bore at $63 \mathrm{~cm}$ from the observer. The projector was running at $72 \mathrm{~Hz}$ with a resolution of $800 \times 600$ and a colour resolution of $3 \times 8$ bit (RBG). The requested frequency of the tapping was about $1 \mathrm{~Hz}$. Seventeen seconds of finger tapping were intermixed with seventeen seconds of rest (Fig. 1). During one scan, this sequence was repeated 5 times resulting in a total of $170 \mathrm{~s}$ scan-time. Subjects were familiarized with the task prior to scanning.

\section{Data analyses}

Group analysis and visualization were achieved using BrainVoyager QX 1.9 (Brain Innovation, Maastricht, The Netherlands). For each of the experimental conditions we modelled a period of $17 \mathrm{~s}$, beginning at the onset of MOVEMENT and REST. Preprocessing included 3D motion correction, slice scan time correction, linear trend removal and spatial smoothing with a Gaussian kernel (full width at half maximum $4 \times 4 \times 4 \mathrm{~mm}$ ). Subsequently, functional datasets were co-registered to the anatomical dataset and transformed into Talairach space. A fixed-effect group analysis was performed using the multi-study, multisubject approach of the general linear model. Group differences in response to MOVEMENT were assessed by contrasting sham and RNS conditions. The obtained $P$ values were corrected for multiple comparisons using Bonferroni correction $(P<0.05)$. 

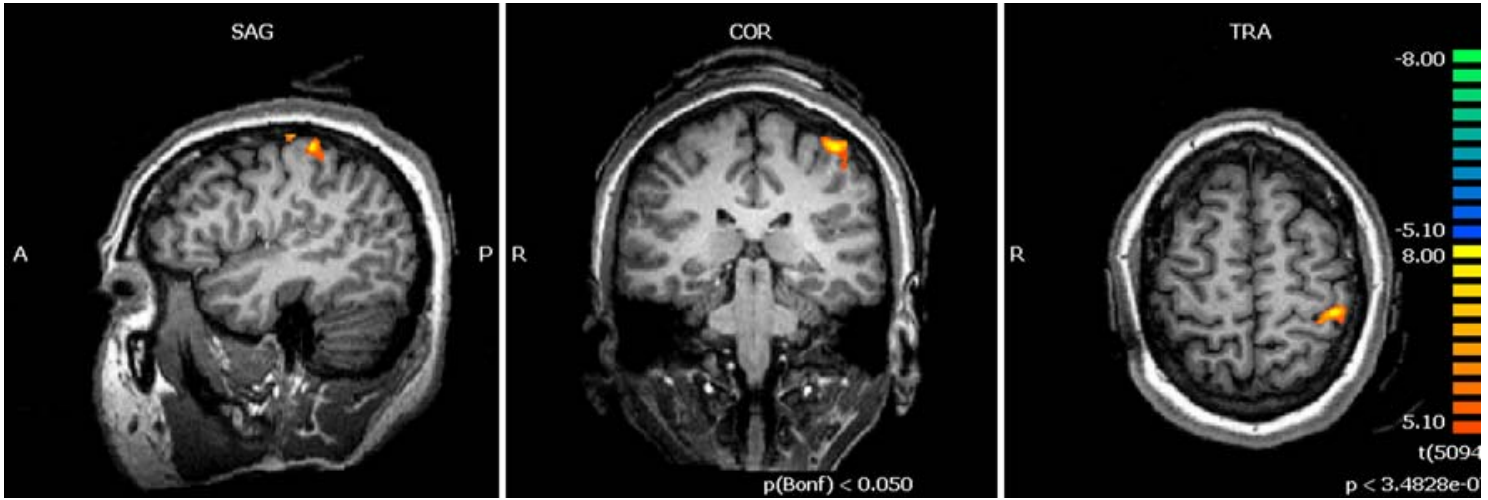

Fig. 2 Activation maps resulting from the contrast of sham and verum stimulation. Difference in activations occurred on the central knob of the left M1. The colour bar at the right corner indicates the $P$ value

Furthermore, the degree of activation was quantified in terms of number of activated voxels per different stimulation conditions contrasted to the REST condition, and was derived from a region-of-interest (ROI) analysis which focused on the left sensorimotor $(x=-40, y=-20$, $z=60)$, premotor $(x=-59, y=0, z=34)$ and supplementary motor cortex (SMA) $(x=-3, y=-9, z=-60)$ in each single subject (Bonferroni correction, $P \leq 0.0001$ ). Statistical evaluations of stimulation condition differences were based on repeated measures of non-parametric Wilcoxon matched pair test $[$ (sham vs. verum stimulation $) \times$ cluster size MOVEMENT].

Additionally, from the signal intensity of the sensorimotor cortex, time courses were extracted for the two different stimulation conditions. Signal changes were calculated as percentage changes in comparison to baseline values, i.e. the three time points directly preceding the onset of MOVEMENT.

\section{Results}

All the subjects tolerated the stimulation well; none reported side-effects during or after the stimulation. None of the subjects were sensitive to the current at either electrode. The functional maps did not suffer from electrode position-induced distortions.

Group analysis revealed activation in the SMA and contralateral sensorimotor and premotor cortices in response to the finger-opposition task. The corresponding main effects for the two stimulation conditions are shown in Fig. 2. For each condition, there was significant activation in the left sensorimotor, premotor area and SMA, even though a difference in the extent and the strength of activation is visible. The extracted signal intensity time courses from the sensorimotor hand-region of interest showed a $10 \%$ decrease after tRNS compared to sham stimulation. The for each pixel showing significant activation (Bonferroni-corrected, $P<0.05)$. Orange to yellow colouring indicates stronger activation after sham stimulation

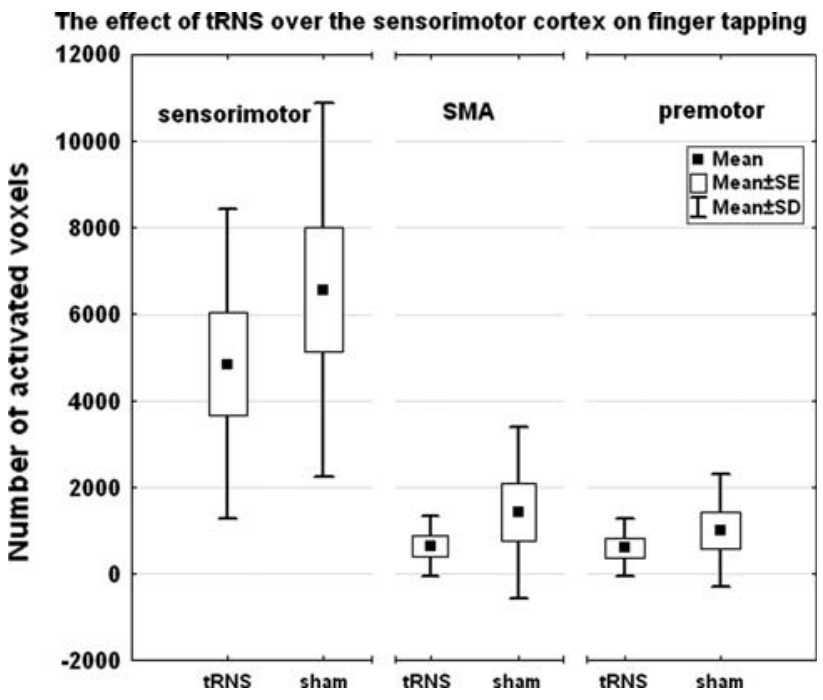

Fig. 3 Boxplots (boxes were constructed using the mean and SEMs, whiskers represent the SDs, $n=9$ ) of the activation volumes resulting from the MOVEMENT after the different stimulation conditions compared to the REST in the sensorimotor, premotor and SMA

assessment of differences between stimulation conditions (Fig. 3) revealed that after tRNS there was a decrease in cluster size compared to the sham condition $(P<0.05)$ at the same threshold. This decrease corresponded to a $17 \%$ change with regard to the sensorimotor cortex when the mean numbers of activated voxels were compared between the two different conditions. Correspondingly, the nonparametric statistical analyses of activation volumes in the sensorimotor cortex of all subjects, revealed a significant influence of the stimulation condition $(T=5, Z=2.07$, $p<0.05$ ). The statistical comparisons demonstrated that after tRNS the MOVEMENT condition showed less activation than after sham stimulation (Fig. 3). This tendency was not significant with regard to the SMA $(T=4, Z=0.94$, $P>0.05)$ and the premotor cortex $(T=3.8, Z=0.8$, $P \geq 0.05)$. 


\section{Discussion}

Here we are able to report that short-duration application of tRNS over the human cortex induces a transient reduction in BOLD response in the sensorimotor cortex of healthy subjects as revealed by a simple finger-tapping task. In our earlier study, we have observed that 10-min tRNS induces consistent excitability increases lasting 90 -min poststimulation. These effects have been observed in 80 subjects through both physiological measures (MEPs) and behavioural tasks (serial reaction time task). If we consider this 4-min stimulation effect as an inhibitory response, the result is at least at first glance, surprising. However, it is possible that different stimulation parameters induce varying changes in levels of cortical excitability. Another study using rTMS by Maeda et al. (2000) reported obtaining two varying responses with the same number of pulses in an rTMS paradigm: an increase in the amplitude of MEPs was observed after 1,600 pulses of $10 \mathrm{~Hz}$ rTMS at $90 \%$ resting motor threshold, but the same effect was not observed by applying 1,600 pulses at $1 \mathrm{~Hz}$. In our study, the attenuating aftereffect on the BOLD responses of a short-duration application of tRNS was observed in all the participants and therefore can be considered robust. However, to further examine the aftereffects of 4-min tRNS, and to make a more direct comparison between the observed short- and long-duration tRNS effects, behavioural data (for example a serial reaction time task, or simple memory task) should be implemented. For example, Terney et al. 2008 used a similar approach when evaluating the aftereffects of tRNS, but for a longer stimulation duration, observing a facilitation of MEPs and transient improvement in an implicit learning task.

A putative mechanism of the observed effect might be related to the homeostatic response of the brain to the consecutive tRNS-motor activation paradigm. It was reported in previous studies that the prior state of cortical activity modified by tDCS influences subsequent practicing of a visuomotor coordination task (e.g. Antal et al. 2008). Indeed, regulatory mechanisms must exist to stabilize the neuronal activity that encompasses both inhibitory and excitatory mechanisms within a dynamic range (Sejnowski 1977). According to the Bienenstock-Cooper-Munro (BCM) rule, a low overall cortical activity level is suggested to enhance the synaptic strength of active neuronal connections, while a high level of activity should diminish it (Bienenstock et al. 1982). According to this rule, if we consider tRNS as an excitatory stimulation, we should expect that a similarly excitability enhancing sensorimotor activity induces the inhibition that results in a decrease in BOLD response.

The neuronal/molecular mechanisms of tRNS are not yet known. Stochastic resonance may play a role in inducing aftereffects, however, at much higher frequency ranges (Grenier et al. 2001). Nevertheless, biological models of stochastic resonance may give an insight into the functionality of the effects that tRNS exerts on central processing mechanisms. There is currently much research devoted to the role of neural synchrony in cognition and perception (for a review see Ward et al. 2006), explaining how a small amount of noise injected into a biological system can enhance the detectability of weak signals. If this is the case, then manipulations of neural oscillations can have farreaching consequences in attentional processing and consciousness. Further experimental data demonstrate that the addition of Gaussian noise to a pulse train stimulus improves signal representation and may normalise neural response patterning in the CNS (Matsuoka et al. 2000). According to these results, increasing neuronal synchronisation and an improved signal detection may result in decreased BOLD-signal (less noise in the system).

However, we have to consider that another possible mechanism of tRNS may be at the level of voltage-gated channels (Bromm 1968; Schoen and Fromherz 2008). According to this hypothesis, tRNS would induce the depolarisation of the neuronal membrane which causes $\mathrm{Na}^{+}$ channels to open. If the $\mathrm{Na}^{+}$entry is insufficient, there is no regenerative depolarisation and thus no action potential, just the "local response". Indeed, previous studies suggest that the BOLD response reflects rather synaptic than spiking activity (Logothetis et al. 2001; Viswanathan and Freeman 2007). Consequently, our results may reflect the change in synaptic activity after tRNS; an issue requiring further studies.

In an earlier study by Baudewig et al. (2001) a 5-min duration of cathodal tDCS at $1 \mathrm{~mA}$ produced a significant inhibitory effect on neuronal excitability in the sensorimotor cortex, using a similar finger-tapping task. They also noted that the brain regions that directly encode for the active performance of the task; the neural finger representations within the sensorimotor hand area, produced identical BOLD MRI responses independently from the application of preceding tDCS. They then concluded that the observed reduction in cortical activity affects other regions; SMA and ipsilateral M1. This indicates that an application of electrical stimulation to the cortex can induce effects somewhat distant from the focally stimulated area. However, a recent study demonstrated that anodal tDCS has only a direct stimulation effect as evaluated by fMRI on the underlying cortex (Kwon et al. 2008).

Nevertheless, tRNS is fundamentally different from tDCS; it possesses no constraint with regard to current orientation. Its unique waveform may influence cortical oscillations and can act at the neuronal membrane. Application of tRNS as measured by fMRI enables the close detection of global and remote stimulatory aftereffects that cannot be 
detected by conventional means of measuring motor cortical excitability, like monophasic TMS. It also allows for the construct of a more accurate active cortical map. This is inline with the aim of treating essential tremor in Parkinson's disease, and could be potentially beneficial in predicting more accurate or therapeutic stimulation loci. The effects of random noise on neuronal excitability and whether it can be therapeutically utilised as a form of brain stimulation, by mechanisms that either activate inhibitory feedback connections or jamming electrical signals within the cortical network, have yet to be outlined (Lozano and Eltahawy 2004). Nevertheless, once we are able to elucidate how tRNS and other varying methods of external electrical stimulation are effective in the mammalian cortex, we may be able to tune these properties into useful therapeutic tools. Many approaches have been taken with the aim of creating potent non-invasive and invasive therapies for the plethora of CNS disturbances (for a review see Benabid et al. 2005). Some have been more successful than others; Bikson et al. (2003) have demonstrated the focal suppression of epileptiform activity by electrical stimulation in the rat hippocampus in vitro.

\section{Conclusion}

We have reported that a short-duration application of tRNS can induce a transient decrease in BOLD activity in the human primary sensorimotor cortex, using a classical finger-tapping task. However, we have yet to understand the mechanisms by which tRNS exerts its effects and whether remote areas of the cortex are affected by its oscillatory nature. Although its mechanism is yet unelucidated, tRNS may prove to be a useful new tool in understanding how neuroplasticity within cortical networks is governed.

Acknowledgements We acknowledge our financial support given by the German Ministry for Education and Science (BMBF) via the Bernstein Centre for Computational Neuroscience (BCCN), Goettingen (Grant No. 01GQ0432).

\section{References}

Antal A, Begemeier S, Nitsche MA, Paulus W (2008) Prior state of cortical activity influences subsequent practicing of a visuomotor coordination task. Neuropsychologia 46(13):3157-3161

Baudewig J, Nitsche MA, Paulus W, Frahm J (2001) Regional modulation of BOLD MRI responses to human sensorimotor activation by transcranial direct current stimulation. Magn Reson Med 45(2):196-201

Benabid AL, Wallace B, Mitrofanis J, Xia R, Piallat B, Chabardes S, Berger F (2005) A putative generalized model of the effects and mechanism of action of high frequency electrical stimulation of the central nervous system. Acta Neurol Belg 105(3):149-157
Bienenstock EL, Cooper LN, Munro PW (1982) Theory for the development of neuron selectivity: orientation specificity and binocular interaction in visual cortex. J Neurosci 2(1):32-48

Bikson M, Hahn PJ, Fox JE, Jefferys JG (2003) Depolarization block of neurons during maintenance of electrographic seizures. J Neurophysiol 90(4):2402-2408

Bromm B (1968) Die Natrium-Gleichrichtung der unterschwellig erregten Membran in der quantitative Formulierung der Ionentheorie. Pflügers Arch 302:233-244

Fregni F, Pascual-Leone A (2007) Technology insight: noninvasive brain stimulation in neurology-perspectives on the therapeutic potential of rTMS and tDCS. Nat Clin Pract Neurol 3(7):383-393

Grenier F, Timofeev I, Steriade M (2001) Focal synchronization of ripples $(80-200 \mathrm{~Hz})$ in neocortex and their neuronal correlates. J Neurophysiol 86:1884-1898

Kwon YH, Ko MH, Ahn SH, Kim YH, Song JC, Lee CH, Chang MC, Jang SH (2008) Primary motor cortex activation by transcranial direct current stimulation in the human brain. Neurosci Lett 435(1):56-59

Logothetis NK, Pauls J, Augath M, Trinath T, Oeltermann A (2001) Neurophysiological investigation of the basis of the fMRI signal. Nature 412(6843):150-157

Lozano AM, Eltahawy H (2004) How does DBS work? Suppl Clin Neurophysiol 57:733-736

Maeda F, Keenan JP, Tormos JM, Topka H, Pascual-Leone A (2000) Interindividual variability of the modulatory effects of repetitive transcranial magnetic stimulation on cortical excitability. Exp Brain Res 133(4):425-430

Matsuoka AJ, Abbas PJ, Rubinstein JT, Miller CA (2000) The neuronal response to electrical constant-amplitude pulse train stimulation: additive Gaussian noise. Hear Res 149(1-2):129-137

Nitsche MA, Paulus W (2000) Excitability changes induced in the human motor cortex by weak transcranial direct current stimulation. J Physiol 527:633-639

Nitsche MA, Paulus W (2001) Sustained excitability elevations induced by transcranial DC motor cortex stimulation in humans. Neurology 57:1899-1901

Nitsche MA, Liebetanz D, Lang N, Antal A, Tergau F, Paulus W (2003) Safety criteria for transcranial direct current stimulation (tDCS) in humans. Clin Neurophysiol 114(11):2220-2222

Oldfield RC (1971) The assessment and analysis of handedness: the Edinburgh inventory. Neuropsychologia 9:97-113

Schoen I, Fromherz P (2008) Extracellular stimulation of mammalian neurons through repetitive activation of $\mathrm{Na}^{+}$channels by weak capacitive currents on a silicon chip. J Neurophysiol 100:346-357

Sejnowski TJ (1977) Statistical constraints on synaptic plasticity. J Theor Biol 69(2):385-389

Terney D, Chaieb L, Moliadze V, Antal A, Paulus W (2008) Increasing human brain excitability by transcranial high-frequency random noise stimulation. J Neurosci 28(52):14147-14155

Viswanathan A, Freeman RD (2007) Neurometabolic coupling in cerebral cortex reflects synaptic more than spiking activity. Nat Neurosci 10(10):1308-1312

Ward LM, Doesburg SM, Kitajo K, MacLean SE, Roggeveen AB (2006) Neural synchrony in stochastic resonance, attention, and consciousness. Can J Exp Psychol 60(4):319-326

Wiesenfeld K, Moss F (1995) Stochastic resonance and the benefits of noise: from ice ages to crayfish and SQUIDs. Nature 373(6509):33-36

Yamamoto Y, Struzik ZR, Soma R, Ohashi K, Kwak S (2005) Noisy vestibular stimulation improves autonomic and motor responsiveness in central neuro-degenerative disorders. Ann Neurol 58:175181 


\title{
2.3 Evaluating Aftereffects of Transcranial Random Noise Stimulation on Cortical Excitability
}

\author{
Chaieb L, Antal A, Paulus W. Under submission in Neural Plasticity
}

Further to the previous studies investigating the aftereffects of transcranial random noise stimulation (tRNS), we have chosen to evaluate the aftereffects of high- frequency tRNS (HFtRNS) (the component of the oscillating spectrum responsible for the reported excitatory aftereffects in the M1) (Terney et al., 2008, Chaieb et al., 2009), at varying stimulation durations and to observe the duration of the longer- term aftereffects. We investigated the 'dose- dependent' effect of stimulation at 4,5 and 6 minute stimulation applications to determine whether there was a 'switching on' of the excitatory aftereffect after a discreet exposure to tRNS. Baseline levels of cortical excitability were measured using monophasic single- pulse TMS, and for all timepoints poststimulation. Significant effects of stimulation duration were found at 5 and 6 minute intervals, but 4 minutes stimulation produced no long- lasting effects. In addition, a 10 minute stimulation duration of HFtRNS produced significant aftereffects lasting 90 minutes poststimulation. We have concluded that tRNS- induced neuroplastic aftereffects may require a minimal stimulation duration in order to activate mechanisms of sustainable LTP- like effects. This study demonstrates the need to optimize tRNS, like other transcranial electric techniques, for a controlled and effective application. 


\title{
Evaluating Aftereffects of Short- duration Transcranial Random Noise Stimulation on Cortical Excitability.
}

\author{
Leila Chaieb, Walter Paulus, Andrea Antal
}

Department of Clinical Neurophysiology, Georg-August University, 37075, Göttingen, Germany

Keywords: Transcranial random noise stimulation (tRNS), primary motor cortex (M1), high frequency stimulation (HFS), neuroplasticity

Running title: tRNS modulates enduring excitability increases

Corresponding author: Leila Chaieb, Department of Clinical Neurophysiology, Georg-August University, Robert-Koch-Str. 40, 37075 Göttingen, Germany, Tel: +49-551-398461, Fax: +49-551-398126, E-mail: Leila.chaieb@med.uni-goettingen.de. 


\section{Abstract}

A 10minute application of high- frequency $(100-640 \mathrm{~Hz})$ transcranial random noise stimulation (tRNS) over the primary motor cortex (M1) increases baseline levels of We have extended previous work demonstrating this effect by decreasing the stimulation duration to 4 , 5 , and 6 minutes to assess whether a shorter duration of tRNS can also induce a change in cortical excitability. Single- pulse monophasic transcranial magnetic stimulation (TMS) was used to measure baseline levels of cortical excitability before and after tRNS. A 5 and6minute tRNS application induced a significant facilitation. 4minute tRNS produced no significant aftereffects on corticospinal excitability. Plastic aftereffects after tRNS on corticospinal excitability require a minimal stimulation duration of 5 minutes. However, the duration of the aftereffect of 5 min tRNS is very short compared to previous studies using tRNS. Developing different transcranial stimulation techniques may be fundamental in understanding how excitatory and inhibitory networks in the human brain can be modulated and how each technique can be optimised for a controlled and effective application. 


\section{Introduction}

Intermittent theta burst stimulation (iTBS) (1), high frequency repetitive transcranial magnetic stimulation (rTMS) (2), anodal transcranial direct current stimulation (tDCS) (3), paired- associative stimulation (PAS) (4) and now high- frequency random noise stimulation (tRNS) (5) are all techniques implemented in order to induce sustained elevations of cortical excitability, when applied over the primary motor cortex (M1).

These methods of transiently modulating neuroplastic- like effects in the human cortex are generally short- lived, and dependent upon the stimulus duration and intensity. In the case of theta burst stimulation and anodal transcranial direct current stimulation aftereffects are well characterised, and here we aim to evaluate the longer aftereffects observed post stimulation with high- frequency transcranial random noise (tRNS).

There is growing interest in understanding the effect of applying transcranial stimulation techniques in intervals of short- duration applications to optimise or prolong aftereffects; this has already been shown with tDCS (6). A study published by our own group reports that both a 10 minute, $1 \mathrm{~mA}$ application of full spectrum (tRNS) or high- frequency transcranial random noise (HF tRNS) over the primary motor cortex (M1) can induce elevations in cortical excitability outlasting the duration of stimulation (5). However, it is yet unknown exactly how long these observable aftereffects are able to endure, whether they are duration dependent and if shorter stimulation durations are still able to modulate transcranial magnetic stimulation (TMS)- induced motor- evoked- potentials (MEPs), a global measure of corticospinal excitability (7). Our aims were to establish whether short duration stimulation applications were able to induce measurable aftereffects, and if so, how long they were able to endure poststimulation. Here we would like to briefly communicate how we have assessed the aftereffects of short- duration stimulation applications of the reported application of tRNS. 


\section{Materials and Methods}

\section{Subjects}

Twenty two healthy subjects (18 male, age-range: $20-30$ years) participated in the study. All participants were informed of all aspects of the experiments and gave written consent. None of the participants suffered from any neurological or psychological disorders, nor had any metal implants or implanted devices; took any relevant medication regularly or prior to their participation. All aspects of the protocol conformed to the Declaration of Helsinki and were approved by the Ethics Committee of the University of Göttingen.

\section{Transcranial random noise stimulation of motor cortex}

tRNS was delivered by a battery-driven electrical stimulator (Version DC-StimulatorPlus, NeuroConn GmbH, Ilmenau, Germany) through conductive-rubber electrodes, placed in two saline-soaked sponges. In the stimulation mode "noise" there is a random level of current generated for every sample (sampling rate $1280 \mathrm{sps}$ ). The random numbers are normally distributed; the probability density function follows a bell-shaped curve. In the frequency spectrum all coefficients have a similar size ("white noise"). The noise signal contains all frequencies up to half of the sampling rate, i.e. a maximum of $640 \mathrm{~Hz}(101 \mathrm{~Hz}-640 \mathrm{~Hz}) \mathrm{Due}$ to the statistical characteristics the signal has no DC offset, provided that the offset is set to zero.

The stimulation electrode was placed over the left motor cortex, which was determined prior to stimulation by single pulse TMS. The reference electrode was placed over the contralateral orbit. The size of the stimulation electrode was $4 \times 4 \mathrm{~cm}$ and the reference electrode was $6 \times 14 \mathrm{~cm}$, and was fixated to the head by elastic bands. High- frequency tRNS was applied for 10 minutes with a current strength of $1000 \mu \mathrm{A}$. The maximal current density was $62.5 \mu \mathrm{A} / \mathrm{cm}^{2}$ over the motor cortex, which is below the safety parameters accepted for 
tDCS (8). The current density was $12 \mu \mathrm{A} / \mathrm{cm}^{2}$ at the electrode placed over the contralateral orbit. For sham stimulation the current was turned up for $30 \mathrm{sec}$ at the beginning of the stimulation, and then subsequently turned off. However, the screen on the stimulator did show the remaining time until the end of the stimulation, as in the verum stimulation condition. Subjects were blinded as to active and sham stimulation conditions.

\section{Measuring corticospinal excitability}

To detect current-driven changes of excitability, motor evoked potentials (MEPs) of the right first dorsal interosseus muscle (FDI) were recorded following stimulation of its motor-cortical representation field by single-pulse TMS. These were induced using a Magstim 200 magnetic stimulator (Magstim Company, Whiteland, Wales, UK), with a figure-of-eight standard double magnetic coil (diameter of one winding, $70 \mathrm{~mm}$; peak magnetic field, $2.2 \mathrm{~T}$; average inductance, $16.35 \mu \mathrm{H})$. Surface electromyogram (EMG) was recorded from the right FDI through a pair of $\mathrm{Ag}-\mathrm{AgCl}$ surface electrodes in a belly-tendon montage. Raw signals were amplified, band-pass filtered $(2 \mathrm{~Hz}-3 \mathrm{kHz}$; sampling rate, $5 \mathrm{kHz})$, digitized with a micro 1401 AD converter (Cambridge Electronic Design, Cambridge, UK) controlled by Signal Software (Cambridge Electronic Design, version 2.13), and stored on a personal computer for offline analysis. Complete relaxation was controlled through auditory and visual feedback of EMG activity and whenever it was necessary, the subject was instructed to relax. The coil was held tangentially to the skull, with the handle pointing backwards and laterally at $45^{\circ}$ from the midline, resulting in a posterior-anterior direction of current flow in the brain. This orientation of the induced electrical field is thought to be optimal for predominantly transsynaptic mode of activation of pyramidal tract neurons synapsing onto the corticospinal system. The optimum position was defined as the site where TMS resulted consistently in the largest MEP 
in the resting muscle. The site was marked with a skin marker to ensure that the coil was held in the correct position throughout the experiment.

\section{Experimental procedure}

Subjects were seated in a comfortable reclining chair with a mounted headrest throughout the experiments. Both experimental conditions were conducted by the same investigator, who was not blinded with regard to stimulation session.

4, 5 and 6minutes Full Spectrum tRNS: Ten (4 min) and twelve (5 and-6 min) subjects participated in two experimental sessions, on separate days, at least 3 days apart to avoid carry over effects. The same subject group participated in the 5 and 6 min stimulation experiments, whilst there was an overlap between the subjects in the group used for the $4 \mathrm{~min}$ experimental session. The subjects received tRN and sham stimulation in a randomised order. Resting motor threshold (RMT), active motor threshold (AMT), the intensity to evoke MEP of $\sim 1 \mathrm{mV}$ peak-to-peak amplitude (SI1mV) and a baseline of TMS-evoked MEPs (40 stimuli) were recorded at $0.25 \mathrm{~Hz}$ prior to stimulation. Stimulus intensities (in percentage of maximal stimulator output) of TMS were determined at the beginning of each experiment. RMT was defined as the minimal output of the stimulator that induced a reliable MEP $(\sim 50 \mu \mathrm{V}$ in amplitude) in at least three of six consecutive trials when the FDI muscle was completely relaxed. AMT was defined as the lowest stimulus intensity at which three of six consecutive stimuli elicited reliable MEP ( $\sim 200 \mu \mathrm{V}$ in amplitude) in the tonically contracting FDI muscle (Rothwell et al., 1999). Immediately following stimulation, 40 single test-pulse MEPs were recorded using monophasic single- pulse TMS at $0.25 \mathrm{~Hz}$, at $0 \mathrm{~min}, 5 \mathrm{~min}, 10 \mathrm{~min}$ poststimulation and then every 10 minutes up to $60 \mathrm{~min}$.

\section{Calculations and statistics}


MEP amplitude (peak-to-peak SI1mV) was automatically calculated by the NuCursor programme (IoN, UCL, London, UK) and the mean value was determined for each timepoint after data had been visually inspected offline, and any MEPs with EMG artefacts were rejected. Repeated measures ANOVAs (CONDITION (4 or 5, 6 min tRNS vs. sham) x TIME (before; $0,5,10,20,30,40,50,60 \mathrm{mins}$, post- stimulation) were used to compare the different stimulation conditions. Effects were considered significant if $\mathrm{p}<0.05$; Bonferroni test was used to see significant diferences between different stimulation condition at a given time point. Student's t-test was used to compare the baseline MEPs among stimulation conditions. All data are given as means + SEM. MEP amplitudes were normalised to baseline; all figures (1-6) show normalised MEP amplitude values.

\section{Results}

All of the subjects tolerated the stimulation; none of the experimental sessions were interrupted due to side effects of the stimulation. The subjects reported no side effects after the stimulation.

4minutes tRNS: 4min tRNS did not induce an excitability increase, as previously observed after 10min tRNS. Indeed, after 4min tRNS there was marked tendency toward inhibition when compared to sham stimulation; however, this short-lived attenuation of cortical excitability was not significant. Repeated measures ANOVA revealed no significant effect of CONDITION $(\mathrm{F}(1,9)=0.4, \mathrm{p}=0.54)$ and $\operatorname{TIME}(\mathrm{F}(8,72)=0.97, \mathrm{p}=0.5)$ (Fig.1). The interaction between CONDITION and TIME was also not significant $(\mathrm{F}(8,72)=1.2, \mathrm{p}=0.31)$.

5 and 6minutes tRNS: 5 and $6 \mathrm{~min}$ tRNS induced an excitability increase, as previously observed after 10min tRNS (5), however, the increase was significant at 5-10min poststimulation. Repeated measures ANOVA revealed significant effect of CONDITION $(\mathrm{F}(2,22)=5.86 ; \mathrm{p}=0.009)$ and $\operatorname{TIME}(\mathrm{F}(8,88)=3.26, \mathrm{p}=0.026)($ Fig.2). The interaction between 
CONDITION and TIME was not significant $(\mathrm{F}(16,176)=1.34, \mathrm{p}=0.1)$. The Bonferroni test revealed significant excitability increases at $5 \mathrm{~min}(\mathrm{p}=0.0005)$, after $6 \mathrm{~min}$ tRNS and at $10 \mathrm{~min}$ $(\mathrm{p}=0.001)$ after 5min tRN stimulation (Fig.3).

RMT, AMT and SI1mV baseline values were compared between tRNS and sham conditions using Student's t-test. There was no significant difference between tRNS and sham stimulation in any of the measurements $(p>0.4)$. Intraindividual differences between participants are also shown, for each stimulation condition (Figs.4,5,6 and 7).

\section{Discussion}

The primary aim of this study was to investigate the stimulation duration threshold at which sustainable neuroplastic effects can be measured, using shorter stimulation applications of full spectrum tRNS, which enabled us to compare the inducible aftereffects of the initial study reporting the facilitatory effects on MEPs of 10mins full spectrum tRNS (5). Full spectrum tRNS was applied in order to compare MEP data with findings from another study reporting an attenuation of the BOLD response after a 4min application of tRNS (9). We have shown that there indeed exists a boundary whereby a minimum stimulation duration is required to obtain enduring aftereffects. Here we report that after 5mins tRNS a significant elevation in MEP amplitudes was observed at 10mins poststimulation. After 6 mins tRNS, a clear trend toward facilitation was observed at 5-10mins and a non-significant increase until 30mins poststimulation. Interestingly, a 4minute tRNS application, also over the M1, did not induce an increase in corticospinal excitability; however, we have reported a blood oxygenated level dependent change after 4 min tRNS in a previous study (9). This may be attributed to differences within the subject population and also the different evaluation methods used.

The observed aftereffects of high frequency tRNS in the present study are similar to those reported after transcranial stimulation with anodal tDCS (3); Anodal tDCS however, has 
one constraint: it is polarity dependent, a factor that does not govern cortical stimulation with tRNS. However, the intensities used to induce after-effects and standard delivery of the stimulation are very similar; even the duration of stimulation in relation to observed aftereffects are not so far apart between these two approaches $(10,5)$.

tRNS induced plasticity may be modulated by the continual activation and rectification of voltage- gated sodium channels (11). Evidence from cultured rat hippocampal neurons suggests that activated inward sodium currents can give rise to weak depolarisations of the cell membrane; the same nonlinearity observed after repetitive high- frequency stimulation, and activation of sodium channels, in hippocampal cells mirror the waveform of the tRNS effect and could account for the sustained excitability enhancing effects of the stimulation (12).

In summary, a relatively short duration (5and 6min) tRNS showed a significant tendency toward facilitation, however, this effect was not as marked as those effects elicited after 10 min tRNS. Contrary to this effect, a 4min tRNS application did not induce an excitability increase. These results suggest that a minimal stimulation duration is critical when aiming to modulate tRNS induced aftereffects. This is also evident in earlier studies examining the inducible aftereffects of tDCS. Nitsche and Paulus (3) reported that a 13minute anodal tDCS application was necessary to produce a sustained excitability increase of 90minutes poststimulation. In contrast 9minute cathodal tDCS induced an excitability diminution lasting up to 60minutes poststimulation (3). We can see that although tRNS is a novel method of inducing sustained elevations of cortical excitability, its aftereffects are comparable with those of more well- established transcranial stimulation techniques. 
Aftereffects of tRNS on cortical excitability

\section{Acknowledgements}

This study was initiated and funded by an unrestricted grant awarded by the Rose Foundation (LC/WP) and by the Bernstein Center for Computational Neuroscience Göttingen (WP) (BMBF 01GQ0782).

\section{Financial Disclosures}

The authors would like to state that they have no conflicts of interest. 


\section{References}

1. Y.Z. Huang, M.J. Edwards, E. Rounis, K.P. Bhatia et al., "Theta burst stimulation of the human motor cortex," Neuron, vol. 45, pp. 201-206, 2005.

2. A. Pascual-Leone, J. Valls-Solé, E.M. Wassermann, et al., "Responses to rapid-rate transcranial magnetic stimulation of the human motor cortex," Brain, vol. 117, no. 4, pp. 847$58,1994$.

3. M.A. Nitsche and W. Paulus, "Sustained excitability elevations induced by transcranial DC motor cortex stimulation in humans," Neurology, vol. 57, pp. 1899-901, 2001.

4. K. Stefan, E. Kunesch, L.G. Cohen, et al., "Induction of plasticity in the human motor cortex by paired associative stimulation," Brain, vol. 123, no. 3, pp. 572-84, 2000.

5. D. Terney, L. Chaieb, V. Moliadze, et al., "Increasing human brain excitability by transcranial high-frequency random noise stimulation," J Neurosci., vol. 28 no. 52, pp. 14147-55, 2008.

6. K.K. Monte-Silva, M.F. Kuo, D. Liebetanz, et al., "Shaping the optimal repetition interval for cathodal transcranial direct current stimulation (tDCS)," J Neurophysiol, no. 103, pp. 1735-1740, 2010.

7. J.C. Rothwell, M. Hallett, A. Berardelli, et al., "Magnetic stimulation: motor evoked potentials: the International Federation of Clinical Neurophysiology," Electroencephalogr Clin Neurophysiol, vol. 52, pp. 97-103, 1999. 
8. M.A. Nitsche, D. Liebetanz, N. Lang, et al., "Safety criteria for transcranial direct current stimulation (tDCS) in humans.” Clin Neurophysiol, vol. 114, no. 11, pp. 2220-2, 2003.

9. L. Chaieb, G. Kovacs, C. Cziraki, et al., "Short-duration transcranial random noise stimulation induces blood oxygenation level dependent response attenuation in the human motor cortex." Exp Brain Res. vol. 198, no. 4, pp. 439-44. 2009.

10. M.A. Nitsche and W. Paulus, "Excitability changes induced in the human motor cortex by weak transcranial direct current stimulation.” J. Physiol. no. 527, pp. 633-639, 2000.

11. B. Bromm, "Die Natrium-Gleichrichtung der unterschwellig erregten Membran in der quantitative Formulierung der Ionentheorie.” Pflügers Arch, no. 302, pp. 233-244, 1968.

12. I. Schoen and P. Fromherz, "Extracellular stimulation of mammalian neurons through repetitive activation of $\mathrm{Na}^{+}$channels by weak capacitive currents on a silicon chip." $J$ Neurophysiol., no. 100, pp. 346-357, 2008. 


\section{Figure Legends}

\section{Figure 1.}

Normalised MEP amplitudes after a 4minute, $1 \mathrm{~mA}$ application of full spectrum tRNS over the human primary motor cortex. There was no significant increase in MEP amplitudes after a moderately short- duration tRNS application. Data are mean peak-to-peak MEP amplitudes over timepoints. Error bars indicate SEM.

\section{Figure 2.}

Normalised MEP amplitudes after a 5minute, $1 \mathrm{~mA}$ application of full spectrum tRNS over the human primary motor cortex. There was a significant increase in MEP amplitudes after a short- duration tRNS application at 10mins poststimulation. Data are mean peak-to-peak MEP amplitudes over timepoints. Error bars indicate SEM. Asterisks denote significant timepoints $(\mathrm{p}<0.05)$

\section{Figure 3.}

Normalised MEP amplitudes after a 6minute, 1mA application of full spectrum tRNS over the human primary motor cortex. There was a significant increase in MEP amplitudes after a short- duration tRNS application at 5 mins poststimulation. Data are mean peak-to-peak MEP amplitudes over timepoints. Error bars indicate SEM. Asterisks denote significant timepoints $(\mathrm{p}<0.05)$.

\section{Figures 4,5,6 and 7.}

Intraindividual differences in corticospinal excitability after tRNS are depicted for 4, 5, 6 min tRNS and sham tRNS at 1mA over time. Each case illustrates the changes in excitability of an individual participant. Data are mean peak-to-peak MEP amplitudes. 
Figure 1.

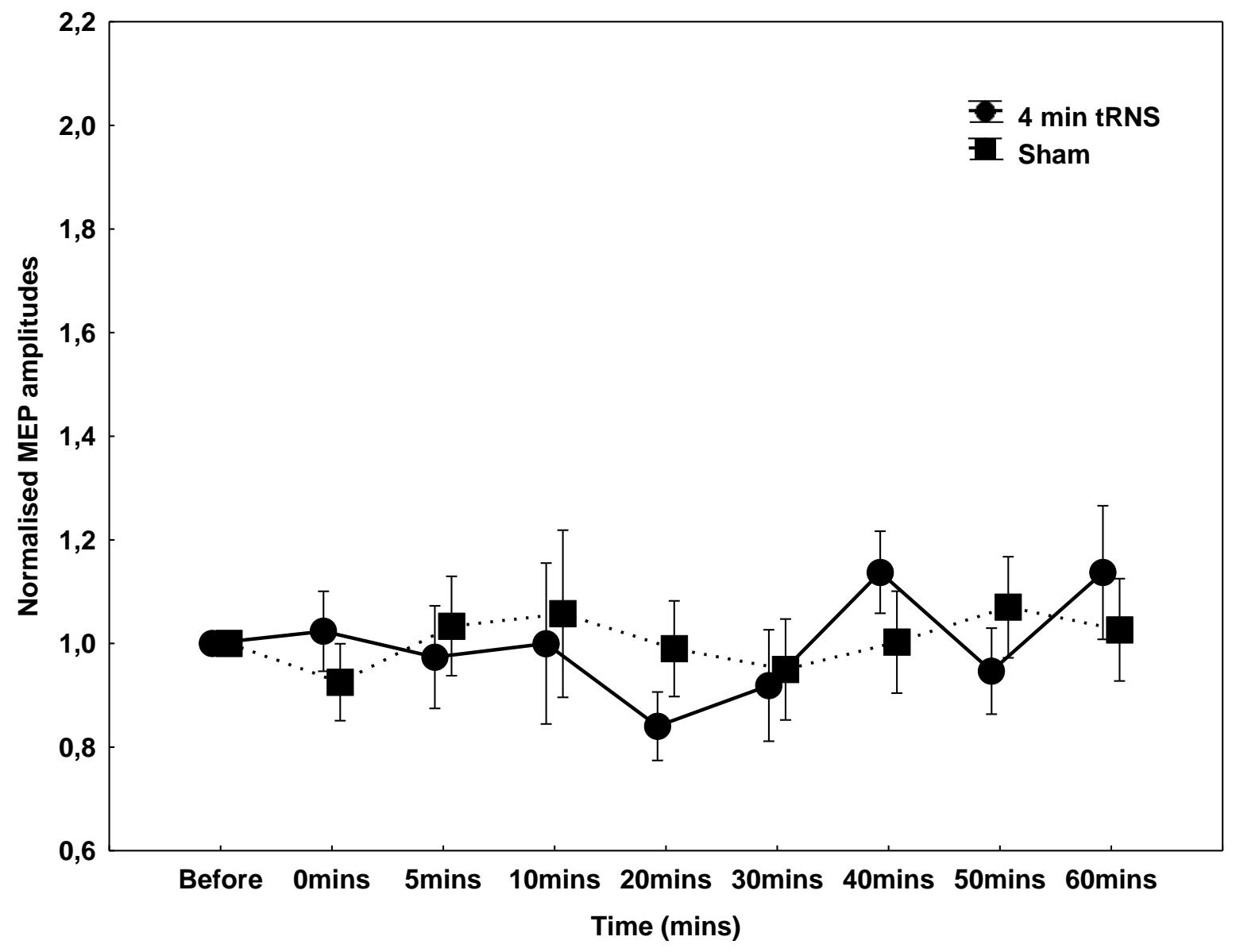


Figure 2.

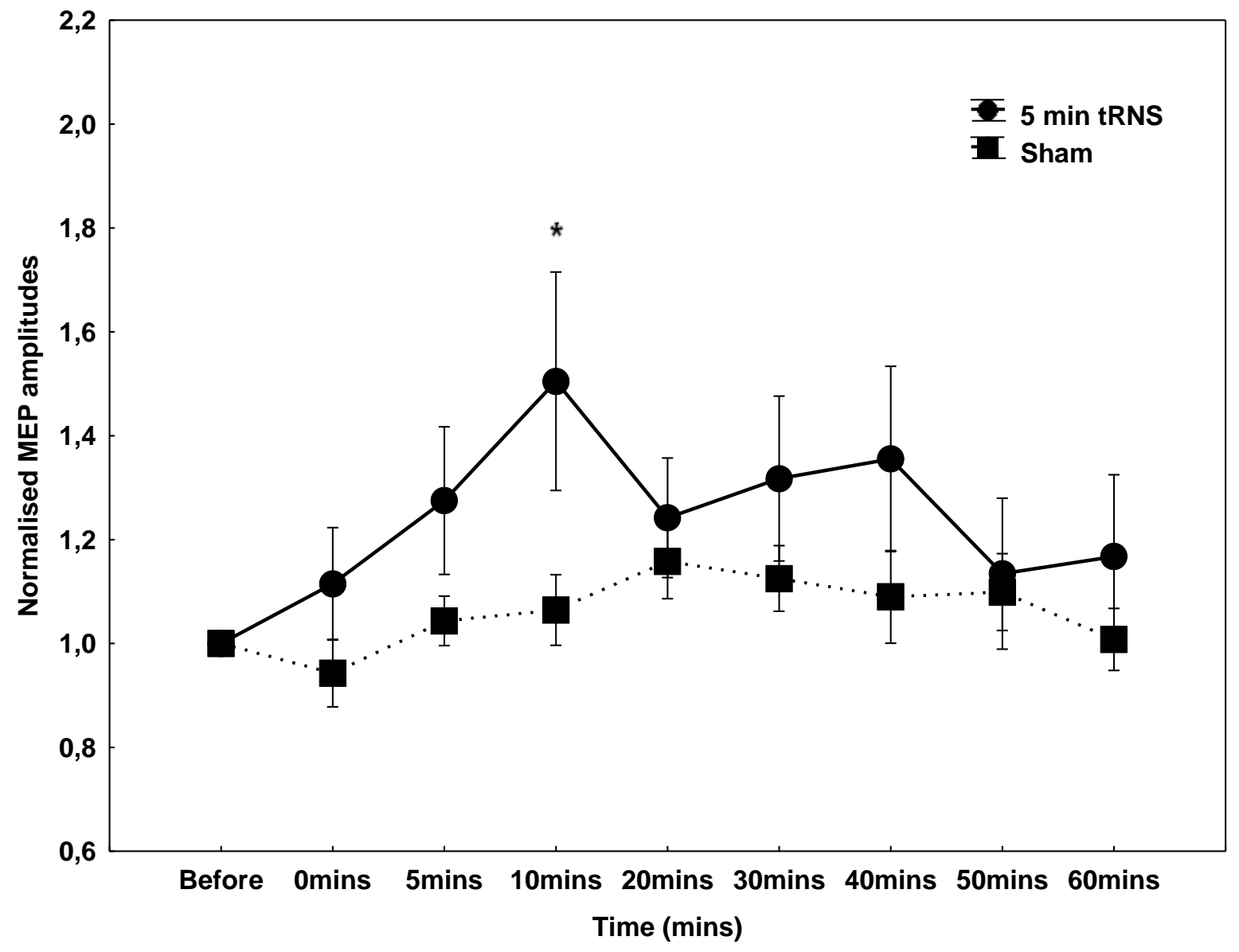


Figure 3.

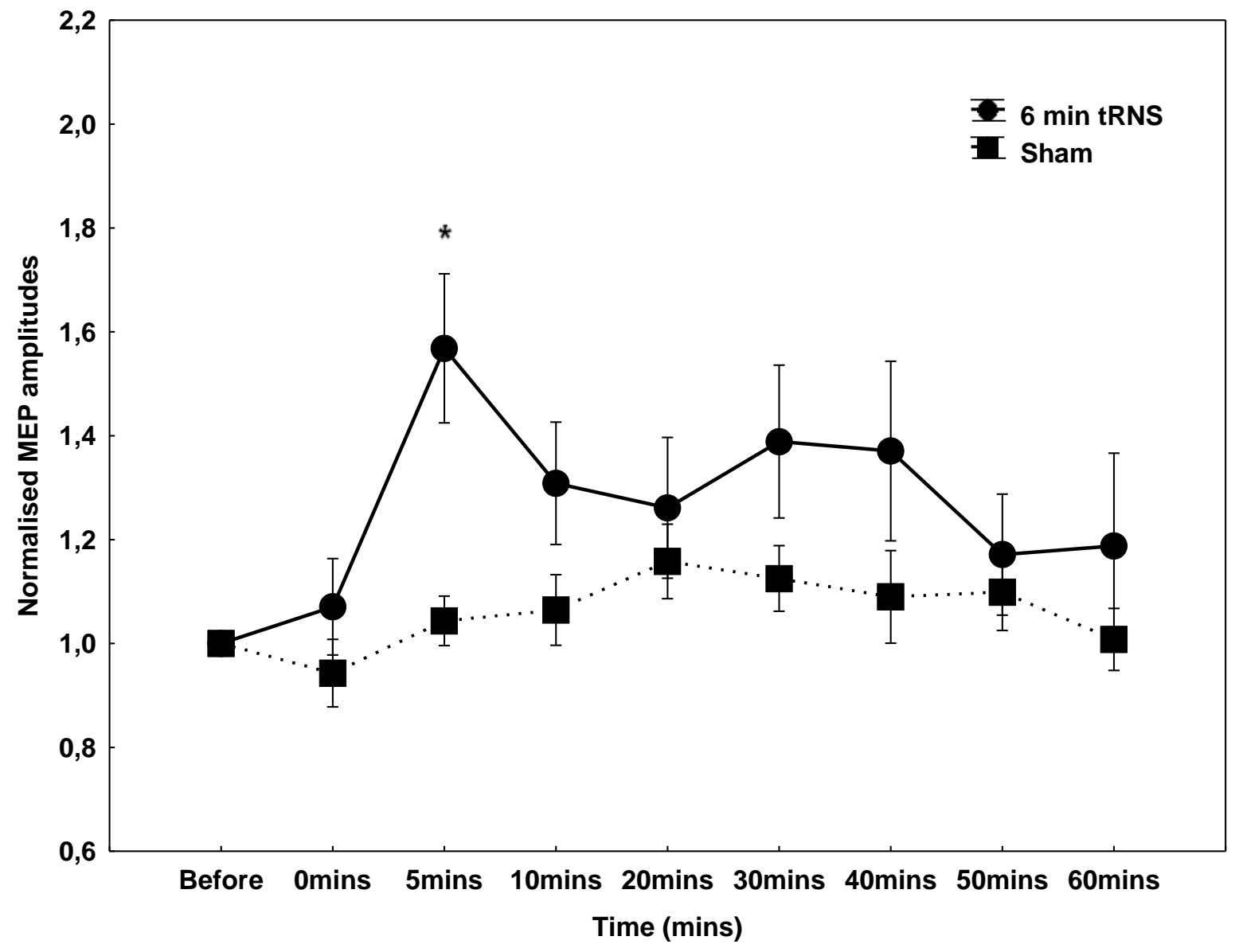


Figure 4.

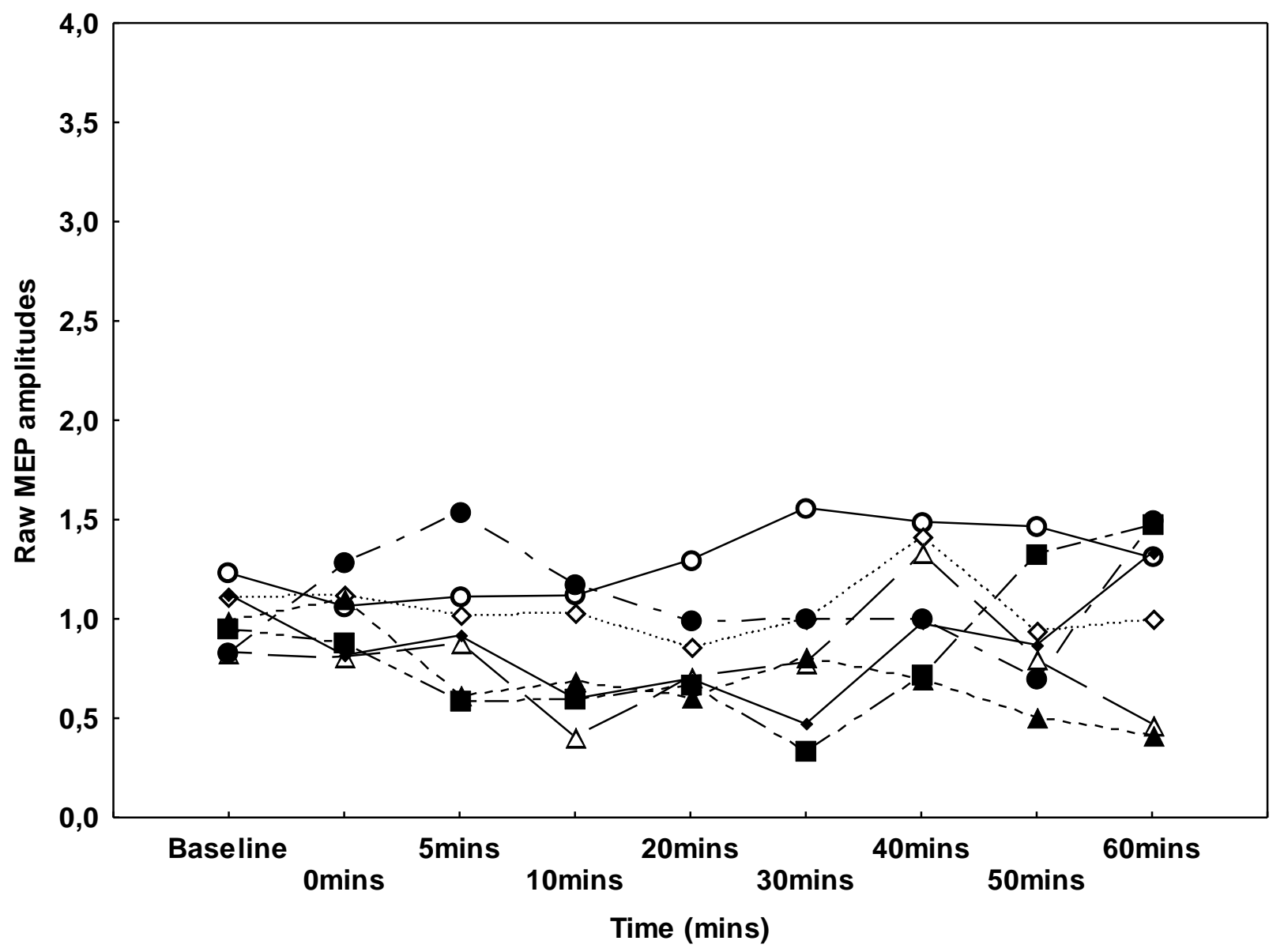




\section{Figure 5.}

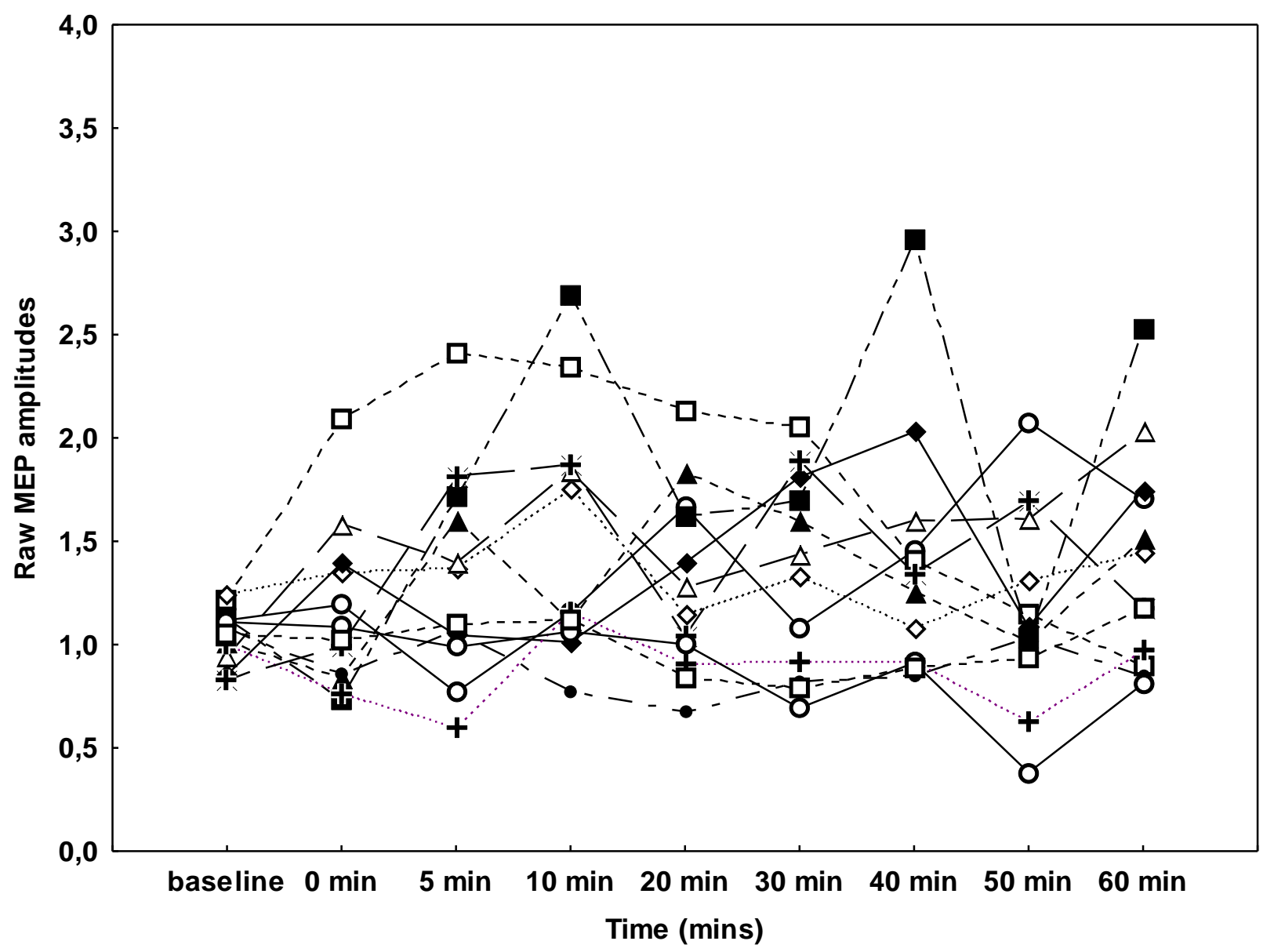


Figure 6.

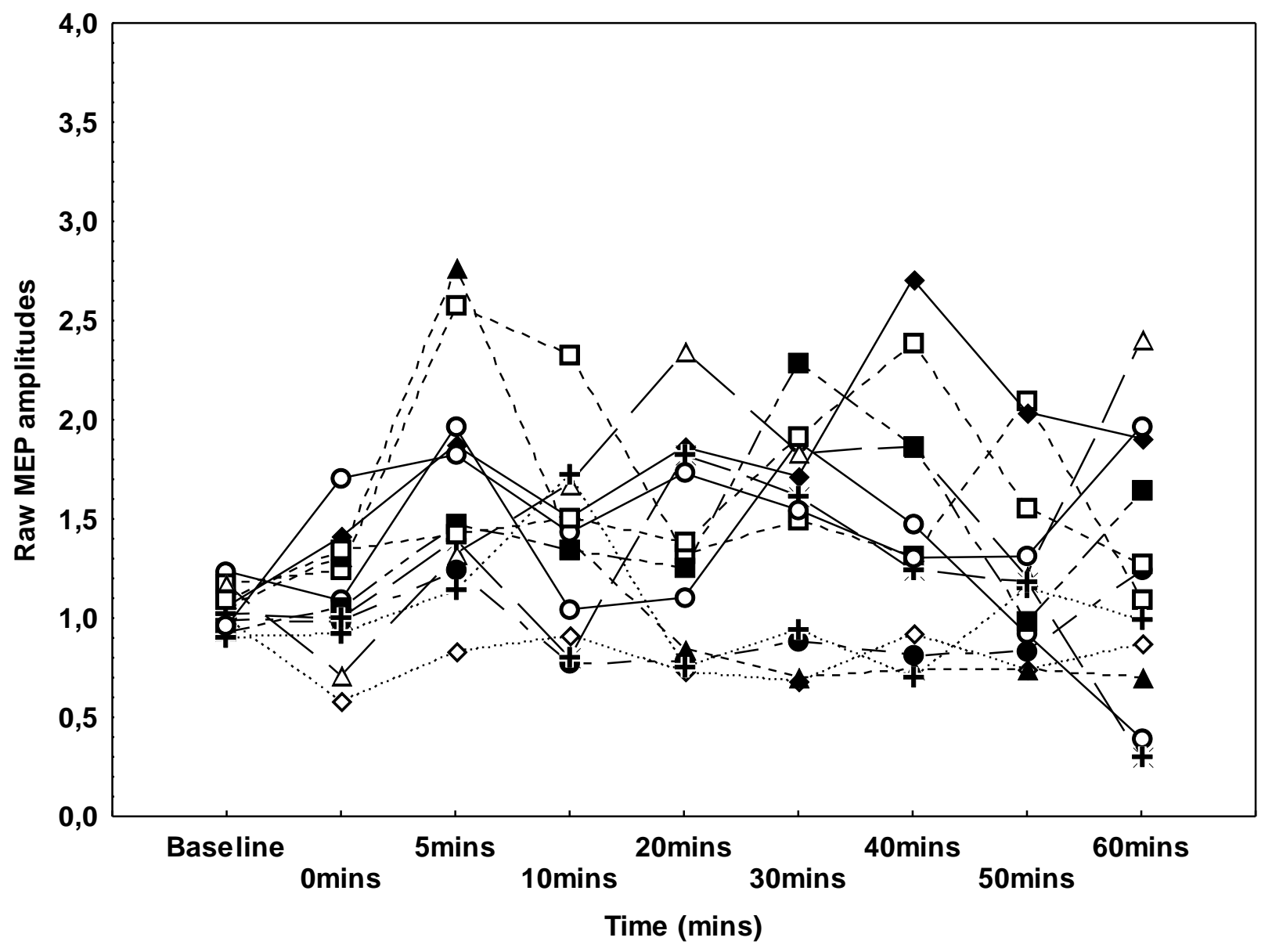


Figure 7.

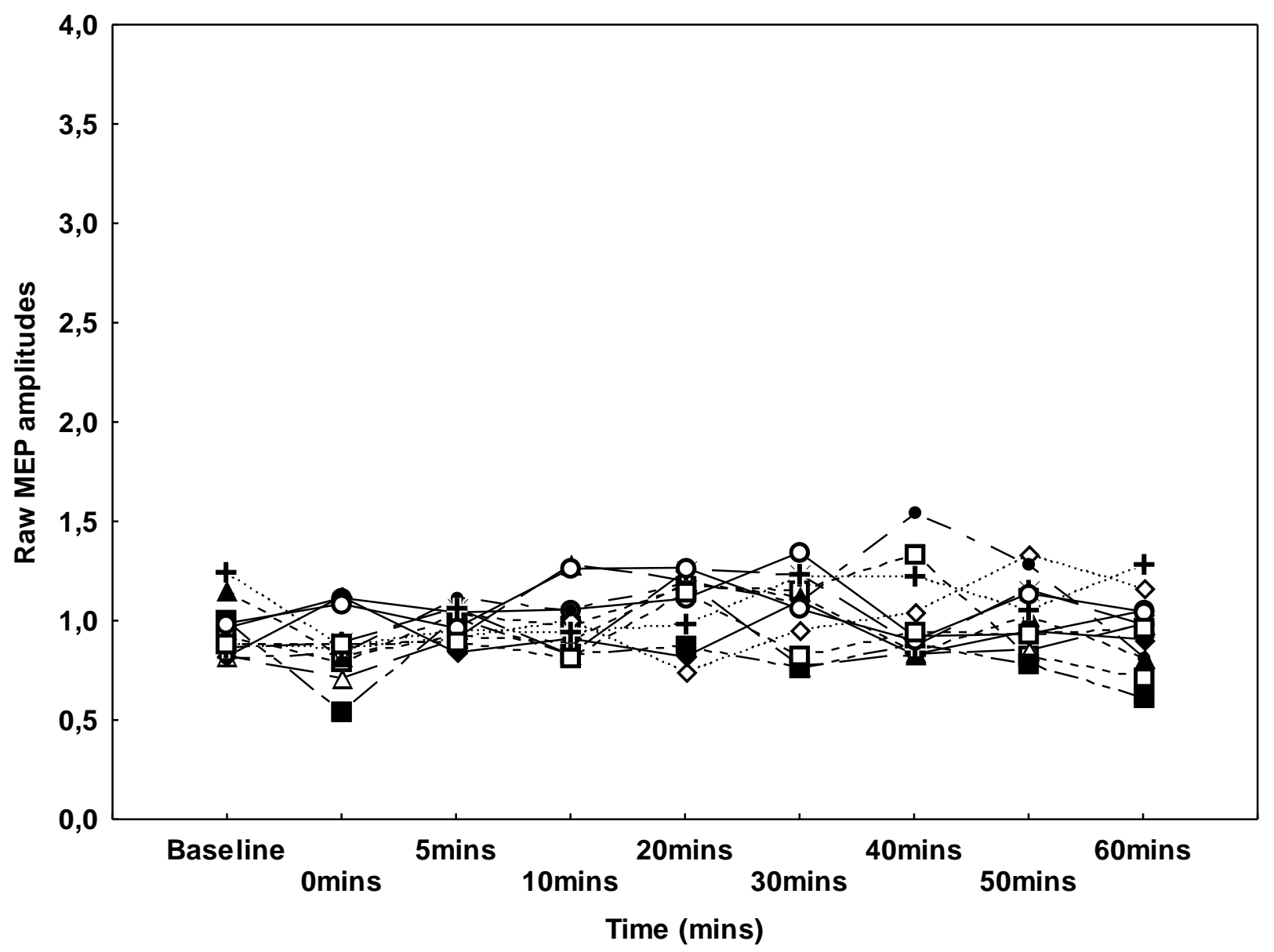




\subsection{Transcranial alternating current stimulation in the low $\mathrm{kHz}$ range increases motor cortex excitability}

Chaieb L, Antal A, Paulus W. Restorative Neurology and Neuroscience; In press.

Transcranial alternating current stimulation (tACS) allows for the fast induction of excitatory effects during and after stimulation. Here we applied an alternating current in the low $\mathrm{kHz}$ range whilst measuring levels of cortical excitability using monophasic single- pulse TMS. We investigated the effects of 1,2 and $5 \mathrm{kHz}$ frequencies of tACS in 11 healthy volunteers who were stimulated for 10 minutes at $1 \mathrm{~mA}$. TMS was applied during tACS stimulation and MEPs were measured from the cortical representation of the right first dorsal interosseous (FDI). In addition, an inactive sham stimulation session was performed with an interval of 4 days during each session. All frequencies increased levels of corticospinal excitability, as measured by the increase in TMSelicited MEP amplitudes, for 30-60 minutes poststimulation. MEP amplitudes were also consistently and significantly increased throughout tACS stimulation. However, 2 and $5 \mathrm{kHz}$ tACS appeared to be more efficacious in inducing sustained changes in corticospinal excitability compared to the $1 \mathrm{kHz}$ and sham conditions. Potential mechanisms of action may centre around the alteration of cortical excitability potentiated via neuronal membrane modulation. 


\section{Transcranial Alternating Current Stimulation in the low kHz Range Increases Motor Cortex Excitability}

Leila Chaieb, Andrea Antal, Walter Paulus

Department of Clinical Neurophysiology, Georg-August University, 37075, Göttingen, Germany

Running title: M1 tACS increases cortical excitation

Abstract: 237 words

Manuscript: 3035

Figures: 5

Corresponding author: Leila Chaieb, Department of Clinical Neurophysiology, Georg-August

University, Robert-Koch-Str. 40, 37075 Göttingen, Germany, Tel: +49-551-398452, Fax:

+49-551-398126, E-mail: Leilachaieb@med.uni-goettingen.de 


\begin{abstract}
Purpose: External transcranial electric and magnetic stimulation techniques allow for the fast induction of sustained and measurable changes in cortical excitability. Here we aim to develop a paradigm using transcranial alternating current (tACS) in a frequency range higher than $1 \mathrm{kHz}$, which potentially interferes with membrane excitation, to shape neuroplastic processes in the human primary motor cortex (M1).
\end{abstract}

Methods: Transcranial alternating current stimulation was applied at 1,2 and $5 \mathrm{kHz}$ over the left primary motor cortex with a reference electrode over the contralateral orbit in 11 healthy volunteers for a duration of $10 \mathrm{~min}$ at an intensity of $1 \mathrm{~mA}$. Monophasic single- pulse transcranial magnetic stimulation (TMS) was used to measure changes in corticospinal excitability, both during and after tACS in the low $\mathrm{kHz}$ range, in the right hand muscle. As a control inactive sham stimulation was performed.

Results: All frequencies of tACS increased the amplitudes of motor- evoked potentials (MEPs) up to 30-60 minutes post stimulation, compared to the baseline. Two and $5 \mathrm{kHz}$ stimulations were more efficacious in inducing sustained changes in cortical excitability than $1 \mathrm{kHz}$ stimulation, compared to sham stimulation.

Conclusions: Since tACS in the low $\mathrm{kHz}$ range appears too fast to interfere with network oscillations, this technique opens a new possibility to directly interfere with cortical excitability, probably via neuronal membrane activation. It may also potentially replace more conventional repetitive transcranial magnetic stimulation (rTMS) techniques for some applications in a clinical setting.

Keywords: primary motor cortex (M1), transcranial magnetic stimulation (TMS), neuroplasticity, transcranial alternating stimulation (tACS), high frequency stimulation (HFS) 


\section{Introduction}

The last decades have established repetitive transcranial magnetic stimulation (rTMS) as an effective tool for modulating and interrupting cortical processing, in addition to treating neurological disturbances like major depression (O'Reardon et al, 2007). Alternative methods such as transcranial direct current stimulation (tDCS) (Nitsche and Paulus, 2000) have also emerged as an indispensable tool to modify cortical plasticity in the human cortex by shifting the resting potential of neuronal membranes (for an overview see Nitsche et al, 2008). When compared with rTMS (Ziemann, 2004) electrical stimulation methods and paradigms have the advantage of being more cost effective, although so far less well investigated.

The emergence of applying oscillating currents to the cerebral cortex not only showed the possibility to induce phosphenes (Kanai et al, 2008) or to interfere with motor performance (Pogosyan et al, 2009); it also allowed the induction of plastic aftereffects (Antal et al, 2008). At present, electrical stimulation methods using alternating currents are currently being explored for a variety of conditions. An attenuation in the proliferation of tumor cells in cell lines, mouse models and patient groups was documented, after being exposed to an electric field of $200 \mathrm{kHz}$ alternating current for $18 \mathrm{hours} /$ day, in both mice models and within a clinical trial (Kirson et al, 2007). A recent study has reported evidence of a reversal of cognitive impairment in the mouse model of Alzheimer's disease after exposure to a $918 \mathrm{MHz}$ electromagnetic field (Arendash et al, 2009).

Here we investigate the effects of tACS in a low $\mathrm{kHz}$ range, clearly outside the range of physiological network oscillations. We have already found that similar neuroplastic aftereffects as seen here are more likely to occur with frequencies between $100 \mathrm{~Hz}$ and 640 $\mathrm{Hz}$ when compared with frequencies below $100 \mathrm{~Hz}$, by using transcranial random noise stimulation (tRNS) (Terney et al, 2008). In the present study we demonstrate that application 
of tACS in the range of $1-5 \mathrm{kHz}$ over the primary motor cortex (M1) can induce significant changes in cortical excitability which outlasts the duration of stimulation.

\section{Materials and methods}

\section{Subjects}

11 healthy participants volunteered for the study ( 5 male, mean age $26.08+/-5.2$ years). All participants were informed as to all aspects of the experimental procedure and all gave written consent. None of the subjects suffered from any neurological disorders, had implanted electrical devices / metallic implants, nor took any relevant medication. One subject was left handed, while all other participants were right handed according to the Edinburgh Handedness Inventory (Oldfield, 1971). All departmental procedures conform to the Declaration of Helsinki, and all experimental protocols were approved by the Ethics Committee of the University of Göttingen.

tACS in the low $\mathrm{kHz}$ range)

tACS was applied over the M1, using a DS5 isolated bipolar constant current stimulator (Digitimer, Welwyn Garden City, UK) connected via a chinch cable to the input of a wave form generator (Peak Tech, Ahrensburg, Germany). Stimulation was delivered via rubber electrodes; one attached to the scalp over the motor cortical representation of the M1, while the other was placed over the contralateral orbit of the right eye. Both electrodes were kept in place with adhesive paste (10/20 conductive EEG paste, Kappamedical, USA.) Stimulation electrodes were $6 \times 8 \mathrm{~cm}\left(48 \mathrm{~cm}^{2}\right)$. Participants were required to keep both the EMG and rubber stimulation electrodes attached to the hand and head montage throughout the experiment. tACS was applied at 1,2 and $5 \mathrm{KHz}$ for a duration of 10 minutes over the left M1, at an intensity of 1mA. For placebo stimulation, no current was applied. 
To detect current-driven changes of excitability, motor evoked potentials (MEPs) of the right first dorsal interosseous (FDI) were recorded following stimulation of its motor-cortical representation field by single-pulse TMS. These were induced using a Magstim 200 magnetic stimulator (Magstim Company, Whiteland, Wales, UK), with a figure-of-eight standard double magnetic coil (diameter of one winding, $70 \mathrm{~mm}$; peak magnetic field, $2.2 \mathrm{~T}$; average inductance, $16.35 \mu \mathrm{H})$. Surface electromyogram (EMG) was recorded from the right FDI through a pair of $\mathrm{Ag}-\mathrm{AgCl}$ surface electrodes in a belly-tendon montage. Raw signals were amplified, band-pass filtered (2 Hz-3kHz; sampling rate, $5 \mathrm{kHz})$, digitized with a micro 1401 AD converter (Cambridge Electronic Design, Cambridge, UK) controlled by Signal Software (Cambridge Electronic Design, version 2.13), and stored on a personal computer for offline analysis. Complete relaxation was controlled through auditory and visual feedback of EMG activity and whenever it was necessary, the subject was instructed to relax. The coil was held tangentially to the skull, with the handle pointing backwards and laterally at $45^{\circ}$ from the midline, resulting in a posterior-anterior direction of current flow in the brain. This orientation of the induced electrical field is thought to be optimal for predominantly transsynaptic mode of activation of pyramidal tract neurons synapsing onto the corticospinal system. The optimum position was defined as the site where TMS resulted consistently in the largest MEP in the resting muscle. The site was marked with a skin marker to ensure that the coil was held in the correct position throughout the experiment.

Experimental design 
Subjects were seated in a comfortable reclining chair with a mounted headrest throughout the experiments. The measurements were always performed by the same investigator. Coil position, angle and proximity to the subjects' scalp were carefully controlled for. Every effort was made to ensure that laboratory conditions for every session were kept the same. They participated in four experimental sessions, on separate days, at least 5 days apart. They received 1, 2 and $5 \mathrm{kHz}$ tACS and sham stimulation in a randomised order. Resting motor threshold (RMT), active motor threshold (AMT), the intensity to evoke MEP of $\sim 1 \mathrm{mV}$ peakto-peak amplitude (SI1mV) and a baseline of TMS-evoked MEPs (30 stimuli) were recorded at $0.25 \mathrm{~Hz}$ prior to stimulation. Stimulus intensities (in percentage of maximal stimulator output) of TMS were determined at the beginning of each experiment. RMT was defined as the minimal output of the stimulator that induced a reliable MEP (50 $\mu \mathrm{V}$ in amplitude) in at least three of six consecutive trials when the FDI muscle was completely relaxed. AMT was defined as the lowest stimulus intensity at which three of six consecutive stimuli elicited reliable MEP (200 $\mu \mathrm{V}$ in amplitude) in the tonically contracting FDI muscle (Rothwell et al, 1999). During stimulation 150 single- pulses were recorded from the left M1 during tACS (and through the stimulating electrode), and then immediately following stimulation, 30 single test-pulse MEPs were recorded at $0.25 \mathrm{~Hz}$, i.e. approximately every 5 mins for half an hour post-stimulation and then at 60 and 90 mins post stimulation. These parameters and experimental procedures were measured at every session for both active tACS and sham stimulation.

\section{Calculations and statistics}

MEP amplitude (peak-to-peak SI1mV) was automatically calculated by the NuCursor programme (IoN, UCL, London, UK) and the mean value was determined for each timepoint after data had been visually analysed offline, and any MEPs with EMG artefacts were rejected. As we aimed to determine the effectiveness of a given verum stimulation compared 
to sham stimulation a comparison among different experiments, with regard to the raw MEP data, was performed using repeated measures ANOVA (TYPE (1 or 2 or $5 \mathrm{kHz}$ versus sham) $\mathrm{x}$ TIME of MEP recordings). Student's $t$-test (paired samples, two tailed) was used to compare MEPs at single time points before and after stimulation between and within groups. A p-value $<0.05$ was considered statistically significant.

\section{Results}

All of the subjects tolerated the stimulation; none of the experimental sessions were interrupted due to side effects of the stimulation. The subjects reported no side effects during or after the stimulation. Subjects were asked to give informal feedback as to the stimulation sensation, and all subjects reported that they were unable to discern whether they had received active or sham stimulation during the experimental sessions.

RMT, AMT and SI1mV baseline values were compared between tACS and sham conditions using Student's-test. There was no significant difference between tACS and sham stimulation in any of the measurements $(\mathrm{p}>0.05)$.

When tACS was applied over the M1 the induced excitability increases rose up to 40$80 \%$, as revealed by single pulse TMS. They lasted for $60-90$ minutes post-stimulation (Fig.1.). According to the Student's $t$-test there was a significant increase in MEPs amplitudes during $1 \mathrm{kHz}$ stimulation at the time points $2-8 \mathrm{~min}(\mathrm{p}=0.002-0.007, \mathrm{t}=2.39-3.9)$ and after stimulation at the time points 20 and $60 \mathrm{~min}(\mathrm{p}=0.03, \mathrm{t}=2.4-2.47)$.

There was a significant increase in MEPs amplitudes during and after $2 \mathrm{kHz}$ stimulation at all of the time points until 90 min post stimulation $(\mathrm{p}=0.0000-0.03, \mathrm{t}=2.5-9.2)$. With regard to $5 \mathrm{kHz}$ stimulation there was a significant increase in MEPs amplitudes during 
and after stimulation at all of the time points until 90 min post stimulation $(\mathrm{p}=0.002-0.05$, $\mathrm{t}=2.3-4.6$ ). Interestingly, there was a significant amplitude increase during sham stimulation at 4 and $6 \min (p=0.01)$ and after stimulation at $20 \min (p=0.03)$.

When $1 \mathrm{kHz}$ and sham stimulation was compared repeated measurements ANOVA revealed no significant main effect of TYPE of stimulation $(F(1,10)=4.4, p=0.06)$, however the factor TIME was significant $(\mathrm{F}(13,130)=2.2, \mathrm{p}=0.01)$. The interaction between TYPE of stimulation and TIME was not significant $(\mathrm{F}(13,130)=1.3, \mathrm{p}=0.2)$. According to the post-hoc analysis, significantly increased MEPs were observed during stimulation at 2-8 min time points compared to sham stimulation $(\mathrm{p}<0.05)$.

When $2 \mathrm{kHz}$ and sham stimulation was compared repeated measurements ANOVA revealed significant main effect of TYPE of stimulation $(\mathrm{F}(1,10)=15.5, \mathrm{p}=0.003)$ and TIME $(\mathrm{F}(13,130)=2.3, \mathrm{p}=0.009)$. The interaction between TYPE of stimulation and TIME was not significant $(\mathrm{F}(13,130)=1.6, \mathrm{p}=0.09)$. According to the post-hoc analysis, significantly increased MEPs were observed during stimulation at 2-8 min time points $(\mathrm{p}<0.05)$ and at 5-15 and 20 min compared to sham stimulation $(\mathrm{p}<0.05)$.

Concerning $5 \mathrm{kHz}$ stimulation repeated measurements ANOVA revealed significant main effect of TYPE of stimulation $(\mathrm{F}(1,10)=10.7, \mathrm{p}=0.01)$ and TIME $(\mathrm{F}(13,130)=1.8$, $\mathrm{p}=0.04)$. The interaction between TYPE of stimulation and TIME was not significant $(\mathrm{F}(13,130)=1.21, \mathrm{p}=0.2)$. According to the post-hoc analysis, significantly increased MEPs were observed during stimulation at $2-10$ min time points $(\mathrm{p}<0.05)$ and after stimulation at 25 , 30 and $90(\mathrm{p}<0.05)$ compared to sham stimulation.

\section{Discussion}


We report here that a $10 \mathrm{~min}$ application of low $\mathrm{kHz}$ tACS over the M1 is able to induce sustained elevations in cortical excitability that outlast the duration of stimulation, compared to sham control. 2 and $5 \mathrm{kHz}$ stimulation was significantly more effective than 1 $\mathrm{kHz}$ stimulation $(2 \mathrm{kHz}$ : $\mathrm{p}=0.0000-0.03 ; 5 \mathrm{kHz}: \mathrm{p}=0.002-0.05$, across time course) compared to the baseline values and to the sham stimulation condition. Single- pulse TMS used during the stimulation to measure changes in cortical excitability has also shown that elevations in the amplitudes of elicited MEPs are clearly evident during stimulation, and so may indicate an entrainment of the targeted neuronal population to the external application of oscillating currents.

It appears that transcranial techniques using higher frequencies aim to amplify and emulate recent studies reporting that the application of high frequency oscillating currents to cell cultures in vitro and animal studies in vivo are effective in entraining neuronal rhythmicity and propagating synchronicity in neuronal networks (Jinno et al, 2007; Radman et al, 2007). The application of a 'blanket' electric field over populations of neurons is able to entrain and modulate neuronal output, which in human subjects may manifest as measurable alterations in cortical excitability (Radman et al, 2007). Many recent studies have sought to investigate whether neuronal networks can be entrained, synchronized or desynchronized by the application of such currents, and whether high frequency oscillations are much more effective in generating synchronization patterns in neuronal assemblies, than those of low frequency oscillations. Application of oscillating currents outside of the physiological range, aiming to interfere with ongoing cortical rhythmicity, however may also be subject to harmonics and/or subharmonics within physiological ranges due to the nature of their alternating cycle. Nevertheless studies suggest that oscillations in a range of several $100 \mathrm{~Hz}$ may still interfere with interneuronal rhythms (Ylinen et al, 1995) whereas frequencies in the $\mathrm{kHz}$ range are much more likely to interfere with membrane excitability. The underlying 
mechanisms resulting in such marked increases in cortical excitability are not yet fully understood but may be related to the jamming of neural transmission and/ or the production and release of low molecular weight proteic neurotransmitters; studies conducted on patients receiving HF deep brain stimulation have also postulated similar mechanisms along with the direct inhibition of spike initiation at the membrane due to activation of inhibitory terminals and retrograde activation of upstream neuronal structures (for a review see Benabid et al, 2005). However, frequencies applied during HF deep brain stimulation are much lower (around $130 \mathrm{~Hz}$ ) than those used in the current study, and so this may also indicate a differing mechanism of action (Benabid et al, 1996). Applications of external currents which cause membrane activation are also accompanied by changes in neurotransmitter release and ion channel potentiation, which in turn lead to changes in protein synthesis (Gartside, 1968; Islam et al, 1995). It may well be that the temporary modification of the synapse once exposed to a rapidly alternating electrical field, alters the associated biochemical mechanisms, such as accumulation of calcium in the presynaptic nerve terminals, and leads to short- term synaptic plasticity effects (Citri and Malenka, 2008).

Another study by Kirson et al, reports that exposure of cell lines to tACS at higher frequencies such as $100 \mathrm{kHz}$ (for mouse melanoma), $200 \mathrm{kHz}$ (for rat glioma) and $150 \mathrm{kHz}$ (for human breast carcinoma) decreased the rate of cell proliferation in targeted tumours; an effect that is associated with two distinct mechanisms: the disruption of mitotic spindle microtubule formation, and the elimination of dividing cells during cleavage. Both of these events are determined by the orientation of the mitosis axis and the AC field vector (Kirson et al, 2004; Kirson et al, 2007). A more recent study has reported evidence of the regression of amyloid- $\beta$ deposition in models of transgenic Alzheimer's disease mice, leading to a reversal in cognitive impairment as measured by a cognitive interference task also after exposure to a $918 \mathrm{MHz}$ electromagnetic field (EMF) (Arendash et al, 2009). Studies investigating the effect 
of mobile phone emissions on cortical excitability and cerebral blood flow have contrasting findings. Haarala et al, 2003 reported that exposure to an active mobile phone produced an immediate decrease in cerebral blood flow bilaterally in the auditory cortex relative to an unexposed area in a study using PET. Subjects were required to perform a visual working memory task during sham and active scanning sessions. No significant results were attributed to the EMF emitted by the active mobile phone. However, Ferreri et al, 2006 demonstrated that the EMF of an active mobile phone modified intracortical excitability during exposure, with short intracortical inhibition (SICI) being reduced and intracortical facilitation (ICF) enhanced in the hemisphere directly exposed, compared to the contralateral non- exposed hemisphere, or to the sham condition.

The advantages in utilizing oscillating currents, especially those of a high frequency, is that potentially neuronal populations can no longer be entrained to the rhythm of an external electric field; with higher frequencies above $1 \mathrm{kHz}$, membranes can be targeted more selectively. Even though this has so far been observed preferentially in vitro at much lower alternating current frequencies and with a uniform field, there is growing evidence that external transcranial techniques may also be able to achieve such pronounced effects (Bikson et al, 2006). Furthermore, compared to tDCS, tACS in general, has the advantage of not being affected by polarity constraints (Antal et al, 2008; Terney et al, 2008). However, transfer of current flow or impact of closely associated brain regions with afferents into the M1 on the aftereffects of stimulation primary somatosensory cortex or supplementary motor area for example, cannot be ruled out. Other studies examining this effect using tDCS or tRNS have also reported this as a factor in aiming to understand how transcranial stimulation techniques have divergent effects in remote areas of the cortex (Chaieb et al, 2009; Lang et al, 2005). Use of paired- pulse TMS measures to investigate the acute intracortical effects of tACS within this $\mathrm{kHz}$ range would enable us to better understand the ways in which tACS differs from 
better characterized transcranial stimulation methods like tDCS. As tACS in the low $\mathrm{kHz}$ range does not influence measures of global corticospinal excitability, like RMT or AMT, this suggests that the mechanism of tACS may be to modulate synaptic activity, as motor thresholds reflect neuronal membrane excitability and are modulated by voltage- gated sodium channel blockers (Ziemann et al, 1996). Within a clinical perspective, external stimulation techniques like tRNS and tACS that are often applied with no noticeable skin sensations (Ambrus et al, 2010), offer the possibility for controlled blinding studies within patient groups, targeting neurological and psychiatric disorders such as depression and stroke (Nitsche et al, 2009; Nowak et al, 2009), disorders currently often targeted by more conventional stimulation techniques like rTMS (Ridding and Rothwell, 2007). tACS aftereffects also compare favorably with those observed poststimulation with tDCS; increases in M1 excitability are able to endure longer after a short stimulation duration. This could potentially provide a preferential therapeutic option, particularly when seeking to target symptoms of neurological disorders or aiding in rehabilitation post trauma. However, the effects of continuous application and long- term use of high frequency transcranial techniques like tACS and tDCS still have yet to be investigated with regards to safety. Animal studies along with recent consensus reports suggest that techniques like TMS are safe and therapeutically relevant but more research is required, especially with regard to use as treatment for neurological and neuropsychiatric disorders (Liebetanz, 2009; Rossi et al, 2009).

\section{Conclusion}

In summary, our preliminary study has sought to evaluate the durable aftereffects of relatively short- duration low kHz tACS over the M1. Stimulation in the low kHz range allows for the generation of similar sustained cortical excitability increases, as previously seen with rTMS or tDCS paradigms. However, they possess a different physiological mode of 
action. rTMS and tDCS will remain the method of choice for inducing inhibitory effects. But for the induction of enduring excitatory aftereffects low kHz tACS offers a new possibility for transcranial electrical stimulation techniques.

\section{Acknowledgements}

This study was initiated and funded by an unrestricted grant awarded by the Rose Foundation (LC/WP) and by the Bernstein Center for Computational Neuroscience Göttingen (WP) (BMBF 01GQ0782).

\section{Financial Disclosures}

The authors would like to state that they have no conflicts of interest. 


\section{References}

Ambrus, G.G., Paulus, W. and Antal, A. (2010). Cutaneous perception thresholds of electrical stimulation methods: Comparison of tDCS and tRNS. Clin Neurophysiol, 121(11), 19081914.

Antal, A., Boros, K., Poreisz, C., Chaieb, L., Terney, D. and Paulus, W. (2008).

Comparatively weak after-effects of transcranial alternating current stimulation (tACS) on cortical excitability in humans. Brain Stim, 1, 97-105.

Arendash, G.W., Sanchez-Ramos, J., Mori, T., Mamcarz, M., Lin, X., Runfeldt, M., Wang, L., Zhang, G., Sava, V., Tan, J. and Cao, C. (2009). Electromagnetic Field Treatment Protects Against and Reverses Cognitive Impairment in Alzheimer's Disease Mice. J Alzheimers Dis, $19,191-210$.

Benabid, A.L., Pollak, P., Gao, D., Hoffmann, D., Limousin, P., Gay, E., Payen, I. and Benazzouz, A. (1996). Chronic electrical stimulation of the ventralis intermedius nucleus of the thalamus as a treatment of movement disorders. J Neurosurg, 84, 203-14.

Benabid, A.L., Wallace, B., Mitrofanis, J., Xia, R., Piallat, B., Chabardes, S. and Berger, F. (2005). A putative generalized model of the effects and mechanism of action of high frequency electrical stimulation of the central nervous system. Acta Neurol Belg, 105, 149-57.

Bikson, M., Radman, T. and Datta, A. (2006). Rational modulation of neuronal processing with applied electric fields. Conf Proc IEEE Eng Med Biol Soc, 1, 1616-9.

Chaieb, L., Kovacs, G., Cziraki, C., Greenlee, M., Paulus, W. and Antal, A. (2009). Shortduration transcranial random noise stimulation induces blood oxygenation level dependent response attenuation in the human motor cortex. Exp Brain Res, 198, 439-44. 
Citri, A. and Malenka, RC. (2008). Synaptic plasticity: multiple forms, functions, and mechanisms. Neuropsychopharma, 33, 18-41.

Ferreri, F., Curcio, G., Pasqualetti, P., De Gennaro, L., Fini, R. and Rossini, P.M. (2006). Mobile phone emissions and human brain excitability. Ann Neurol, 60, 188-96.

Gartside, B. (1968). Mechanisms of sustained increases of firing rate of neurones in the rat cerebral cortex after polarization: role of protein synthesis. Nat, 220, 383-384.

Haarala, C., Aalto, S., Hautzel, H., Julkunen, L., Rinne, J.O., Laine, M., Krause, B. and Hämäläinen, H. (2003). Effects of a $902 \mathrm{MHz}$ mobile phone on cerebral blood flow in humans: a PET study. Neurorep, 14, 2019-23.

Islam, M., Aftabuddin, A., Moriwaki, Y., Hattori, Y. and Hori, Y. (1995). Increase in the calcium level following anodal polarization in the rat brain. Brain Res, 684, 206-208.

Jinno, S., Klausberger, T., Marton, L.F., Dalezios, Y., Roberts, J.D., Fuentealba, P., Bushong, E.A., Henze, D., Buzsáki, G., and Somogyi, P. (2007). Neuronal diversity in GABAergic long-range projections from the hippocampus. J Neurosci, 27, 8790-804.

Kanai, R., Chaieb, L., Antal, A., Walsh, V. and Paulus, W. (2008). Frequency-dependent electrical stimulation of the visual cortex. Curr Biol, 18, 1839-1843.

Kirson, E.D., Dbalý, V., Tovarys, F., Vymazal, J., Soustiel, J.F., Itzhaki, A., Mordechovich, D., Steinberg-Shapira, S., Gurvich, Z., Schneiderman, R., Wasserman, Y., Salzberg, M., Ryffel, B., Goldsher, D., Dekel, E. and Palti, Y. (2007). Alternating electric fields arrest cell proliferation in animal tumor models and human brain tumors. Proc Natl Acad Sci USA, 104, 10152-7. 
Kirson, E.D., Gurvich, Z., Schneiderman, R., Dekel, E., Itzhaki, A., Wasserman, Y., Schatzberger, R., and Palti, Y. (2004). Disruption of cancer cell replication by alternating electric fields. Cancer Res, 64, 3288-95.

Lang, N., Siebner, H.R., Ward, N.S., Lee, L., Nitsche, M.A., Paulus, W., Rothwell, J.C., Lemon, R.N. and Frackowiak, R.S. (2005). How does transcranial DC stimulation of the primary motor cortex alter regional neuronal activity in the human brain? Eur J Neurosci, 22, 495-504.

Liebetanz, D., Koch, R., Mayenfels, S., König, F., Paulus, W. and Nitsche, M.A. (2009). Safety limits of cathodal transcranial direct current stimulation in rats. Clin Neurophysiol, $120,1161-7$

Nitsche, M.A. and Paulus, W. (2000). Excitability changes induced in the human motor cortex by weak transcranial direct current stimulation. J. Physiol, 527, 633-639.

Nitsche, M.A., Boggio, PS., Fregni, F. and Pascual-Leone, A. (2009). Treatment of depression with transcranial direct current stimulation (tDCS): a review. Exp Neurol, 219, 149.

Nitsche, M.A., Cohen, L.G., Wassermann, E.M., Priori, A., Lang, N., Antal, A., Paulus, W., Hummel, F., Boggio, P.S., Fregni, F. and Pascual-Leone, A. (2008). Transcranial direct current stimulation: State of the art 2008. Brain Stim, 1, 206-23.

Nowak, DA., Grefkes, C., Ameli, M. and Fink, G.R. (2009). Interhemispheric competition after stroke: brain stimulation to enhance recovery of function of the affected hand. Neurorehabil Neural Rep, 23, 641-56. 
Oldfield, R. (1971). The assessment and analysis of handedness: the Edinburgh inventory. Neuropsycho, 9, 97-113.

O'Reardon, J.P., Solvason, H.B., Janicak, P.G., Sampson, S., Isenberg, K.E., Nahas, Z., McDonald, W.M., Avery, D., Fitzgerald, P.B., Loo, C., Demitrack, M.A., George, M.S. and Sackeim, H.A. (2007). Efficacy and safety of transcranial magnetic stimulation in the acute treatment of major depression: a multisite randomized controlled trial. Biol Psych, 62, 120816.

Pogosyan, A., Gaynor, LD., Eusebio, A. and Brown, P. (2009). Boosting cortical activity at Beta-band frequencies slows movement in humans. Curr Biol, 19, 1637-1641.

Radman, T., Datta, A. and Peterchev, AV. (2007). In vitro modulation of endogenous rhythms by AC electric fields: Syncing with clinical brain stimulation. J Physiol, 584, 369-70.

Radman, T., Su, Y., An, JH., Parra, LC. and Bikson, M. (2007). Spike timing amplifies the effect of electric fields on neurons: implications for endogenous field effects. J Neurosci, 27, 30306.

Ridding, MC. and Rothwell, JC. (2007). Is there a future for therapeutic use of transcranial magnetic stimulation? Nat Rev Neurosci, 8, 559-67.

Rossi, S., Hallett, M., Rossini, PM. and Pascual-Leone, A. (2009). Safety, ethical considerations, and application guidelines for the use of transcranial magnetic stimulation in clinical practice and research. Safety of TMS Consensus Group. Clin Neurophysiol, 120, 2008-39.

Rothwell, J.C., Hallett, M., Berardelli, A., Eisen, A., Rossini, P. and Paulus, W. (1999). Magnetic stimulation: motor evoked potentials: the International Federation of Clinical Neurophysiology. Electroencephalogr Clin Neurophysiol Suppl, 52, 97-103. 
Terney, D., Chaieb, L., Moliadze, V., Antal, A. and Paulus, W. (2008). Increasing human brain excitability by transcranial high-frequency random noise stimulation. $J$ Neurosci, 28 , $14147-14155$.

Ylinen, A., Bragin, A., Nádasdy, Z., Jandó, G., Szabó, I., Sik, A., and Buzsáki, G. (1995). Sharp wave-associated high-frequency oscillation $(200 \mathrm{~Hz})$ in the intact hippocampus: network and intracellular mechanisms. J Neurosci, 15, 30-46.

Ziemann, U., Lönnecker, S., Steinhoff, B.J. and Paulus, W. (1996). Effects of antiepileptic drugs on motor cortex excitability in humans: a transcranial magnetic stimulation study. Ann Neurol, 40, 367-78.

Ziemann, U. (2004). TMS induced plasticity in human cortex. Rev Neurosci, 15, 253-66. 


\section{Figure legends}

Figure 1: Effect of 10 min application of tACS on the M1, during stimulation and time course poststimulation. Effects of 1,2 and $5 \mathrm{kHz}$ on cortical excitability of M1, as measured by mean MEP amplitude. MEP values are normalised to baseline. Timecourse over 90mins is shown. +/- error bars indicate SEM.

Figure 2A: Intraindividual responses to Sham tACS on M1 excitability poststimulation. Intraindividual responses to tACS on M1 excitability as measured by mean MEP amplitude. Timecourse over 90mins is shown.

Figure 2B: Intraindividual responses to $1 \mathrm{kHz}$ tACS on M1 excitability poststimulation. Intraindividual responses to tACS on M1 excitability as measured by mean MEP amplitude. Timecourse over 90mins is shown.

Figure 2C: Intraindividual responses to $2 \mathrm{kHz}$ tACS on M1 excitability poststimulation. Intraindividual responses to tACS on M1 excitability as measured by mean MEP amplitude. Timecourse over 90mins is shown.

Figure 2D: Intraindividual responses to $5 \mathrm{kHz}$ tACS on M1 excitability poststimulation. Intraindividual responses to tACS on M1 excitability as measured by mean MEP amplitude. Timecourse over 90mins is shown. 
Figure 1: Effect of 10 min application of tACS on the M1, during stimulation and time course poststimulation.

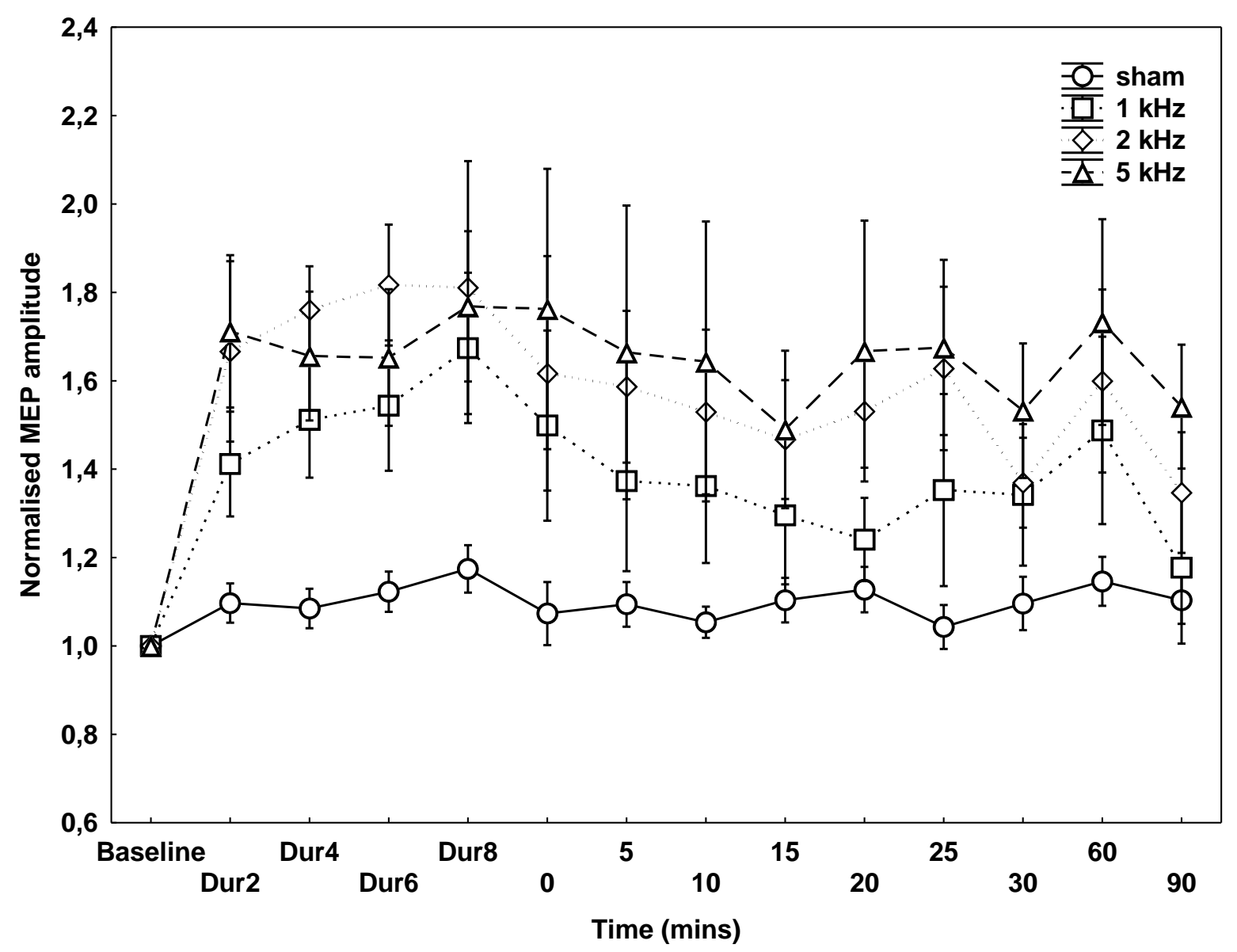


Figure 2A: Intraindividual responses to Sham tACS on M1 excitability poststimulation.

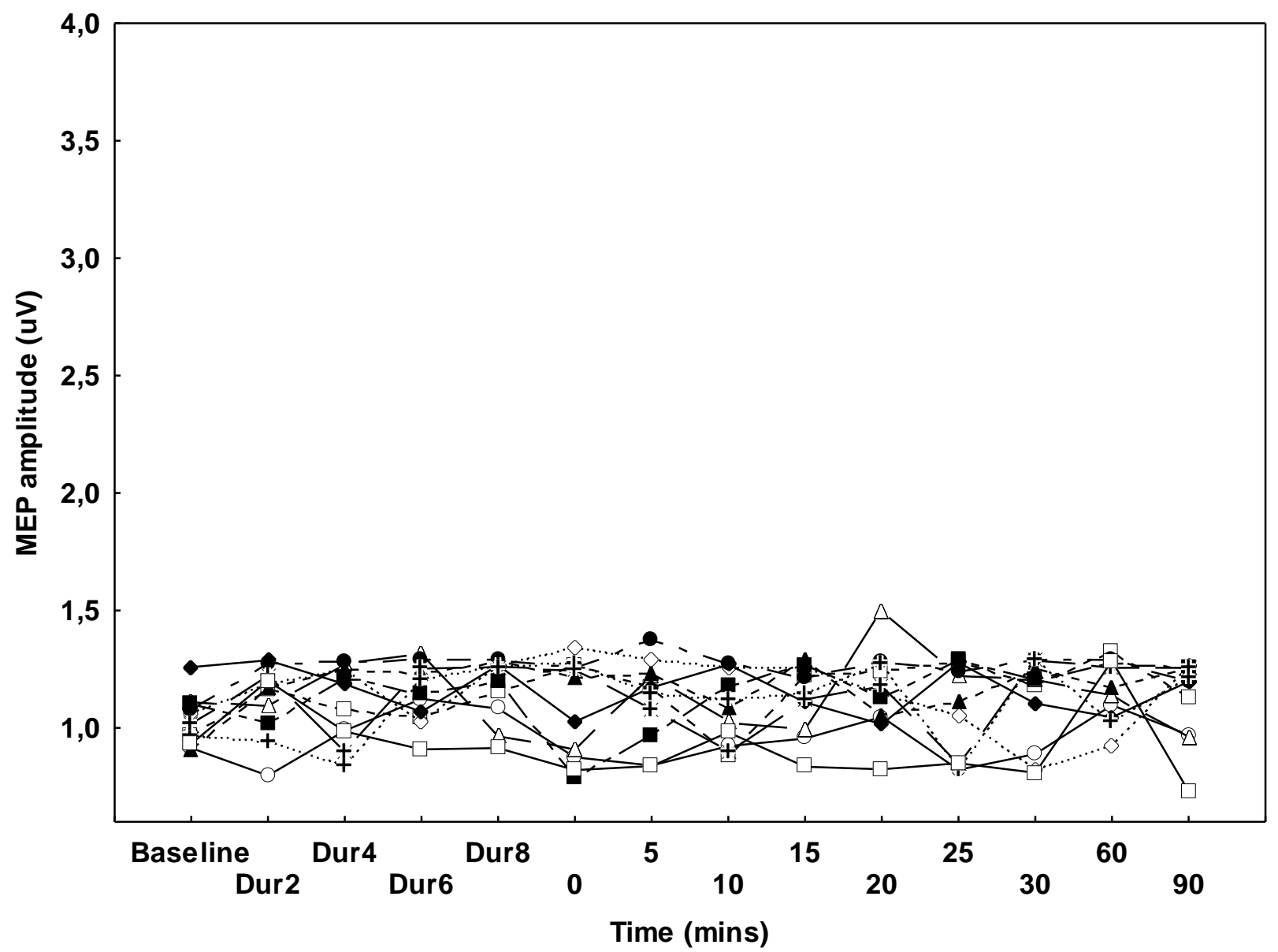


Figure 2B: Intraindividual responses to $1 \mathrm{kHz}$ tACS on M1 excitability poststimulation.

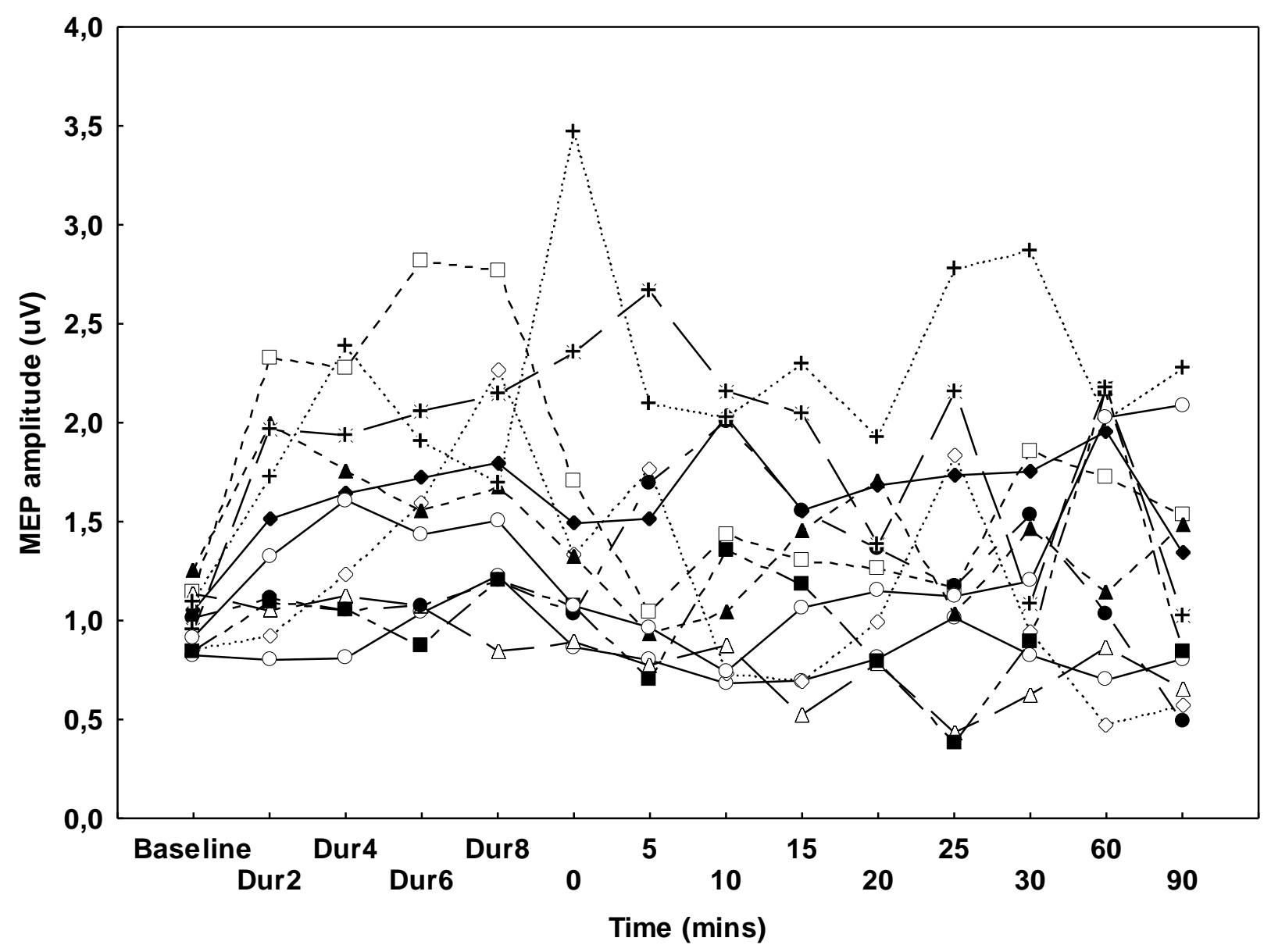


Figure 2C: Intraindividual responses to $2 \mathrm{kHz}$ tACS on M1 excitability poststimulation.

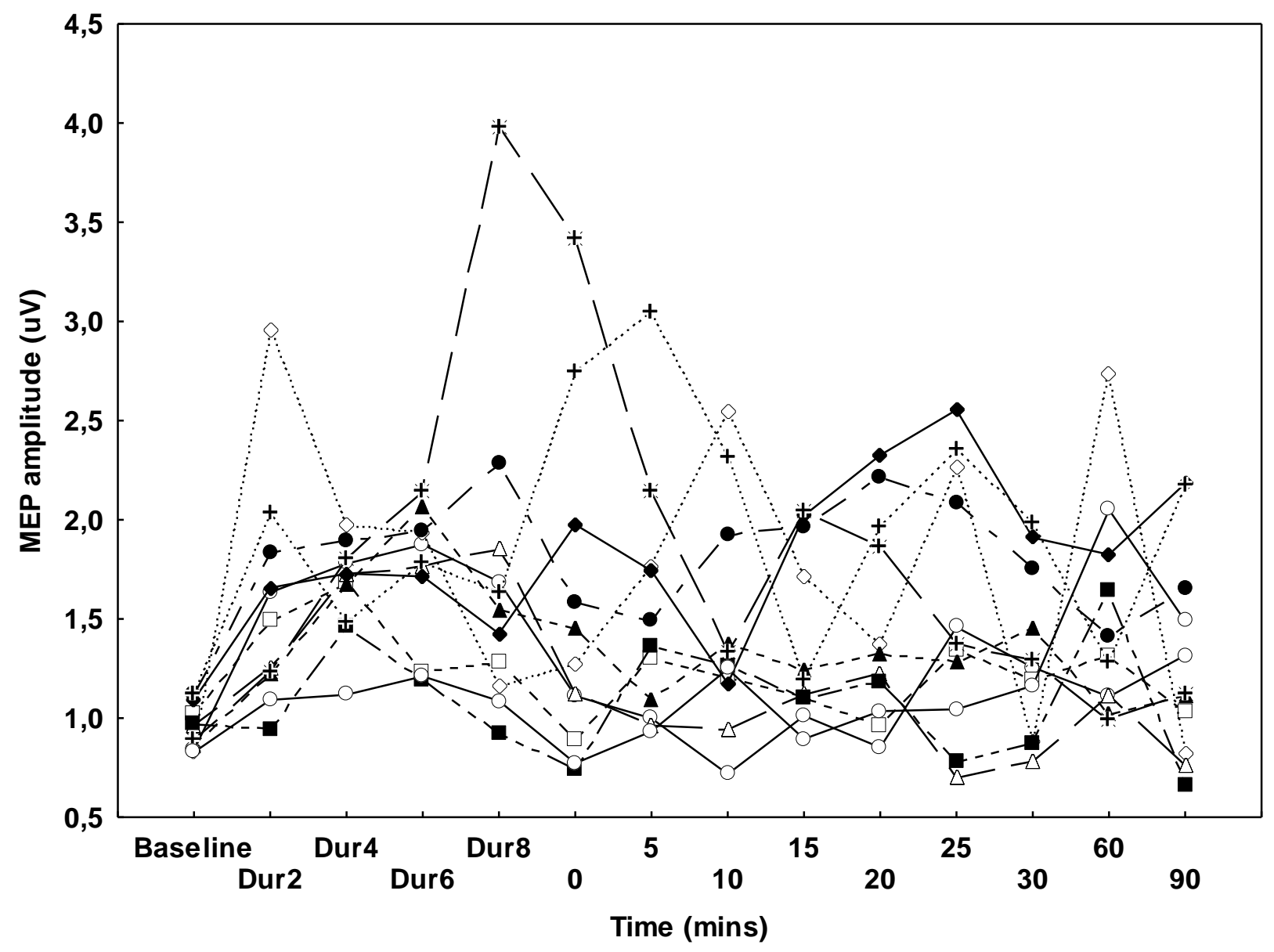


Figure 2D: Intraindividual responses to $5 \mathrm{kHz}$ tACS on M1 excitability poststimulation.

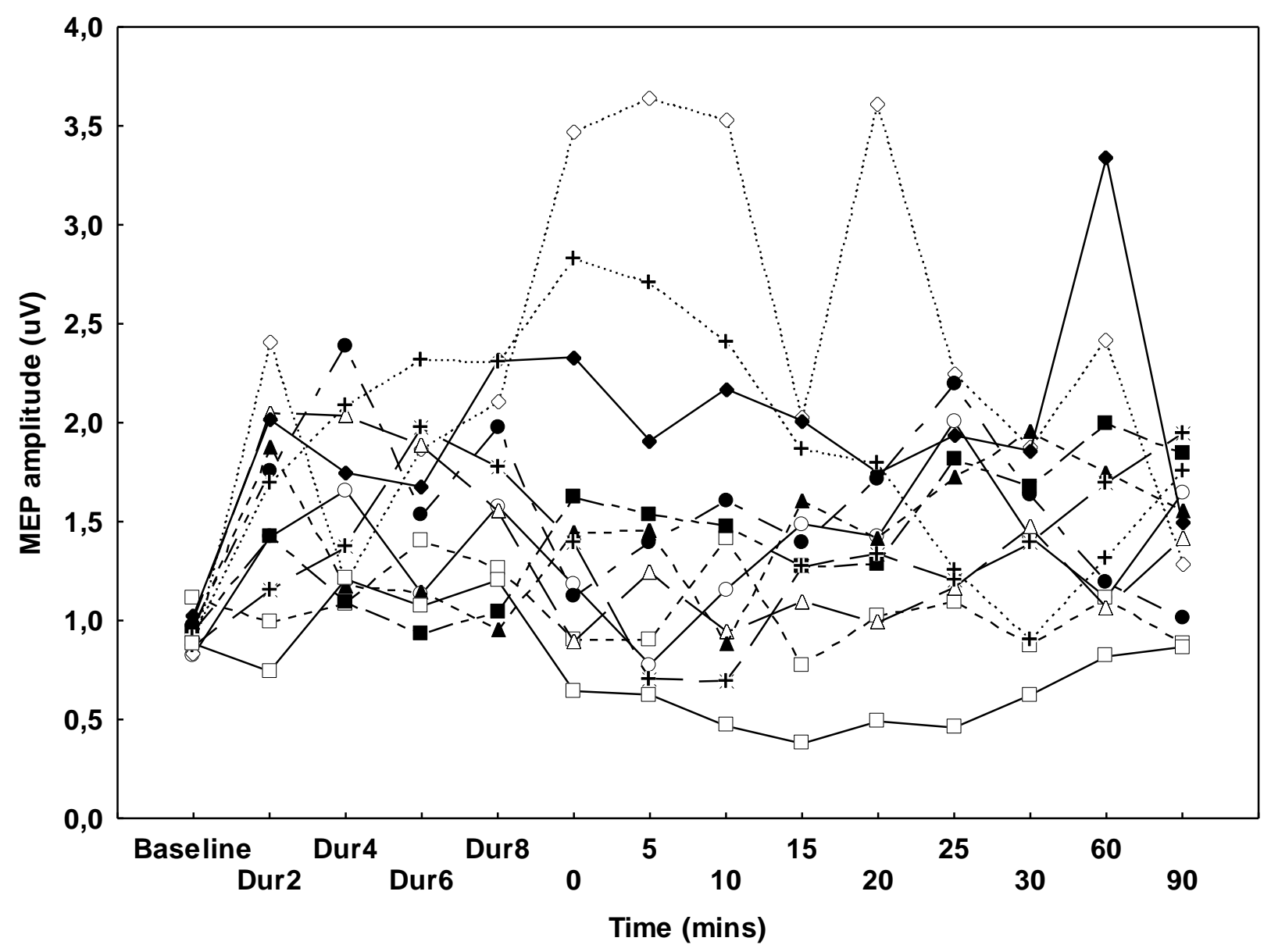




\subsection{Comparatively weak after-effects of transcranial alternating current stimulation (tACS) on cortical excitability in humans}

Antal, A., Boros, K., Poreisz, C., Chaieb, L., Terney, D. \& Paulus, W. Brain Stimulation 2008; 1:97-105.

The first study detailing the effects of tACS and sinusoidal DC stimulation (tSDCS) on the human primary motor cortex; TMS, electroencephalogram (EEG) and a variant of the serial reaction time task (SRTT) were used to evaluate the effects of tACS in healthy human volunteers. Frequencies of 1, 10, 15, 30 and $45 \mathrm{~Hz}$ were applied for 2-10 minutes over the M1, depending upon the experimental paradigm used, to detect changes in cortical excitability with monophasic single- pulse TMS. No significant changes in excitability were observed using TMS and EEG measures; however, under a $10 \mathrm{~Hz}$ tACS application, a transient improvement in motor learning was detected during performance of the SRTT. Results suggest that tACS at a lower intensity than $1 \mathrm{~mA}$ produced effects that were too weak to be detected using TMS and EEG. However, the transient improvement during the SRTT demonstrates that tACS is interacting with ongoing brain rhythmicity. Stimulation parameters therefore need to be optimized and fully characterised before tACS can be used as an effective method of inducing sustained and measureable changes in cortical excitability. 


\title{
Comparatively weak after-effects of transcranial alternating current stimulation (tACS) on cortical excitability in humans
}

\author{
Andrea Antal, $\mathrm{PhD}^{\mathrm{a}}$, Klára Boros, MD ${ }^{\mathrm{a}}$, Csaba Poreisz, MD ${ }^{\mathrm{a}}$, Leila Chaieb, $\mathrm{MS}^{\mathrm{a}}$, \\ Daniella Terney, MD ${ }^{a, b}$, Walter Paulus, MD ${ }^{a}$
}

${ }^{a}$ Department of Clinical Neurophysiology, Georg-August University, Göttingen, Germany

${ }^{b}$ Department of Neurology, University of Szeged, Szeged, Hungary

\begin{abstract}
Objective
Interference with brain rhythms by noninvasive transcranial stimulation that uses weak transcranial alternating current may reveal itself to be a new tool for investigating cortical mechanisms currently unresolved. Here, we aim to extend transcranial direct current stimulation (tDCS) techniques to transcranial alternating current stimulation (tACS).
\end{abstract}

\section{Background}

Parameters such as electrode size and position were taken from those used in previous tDCS studies.

\section{Methods}

Motor evoked potentials (MEPs) revealed by transcranial magnetic stimulation (TMS), electroencephalogram (EEG)-power, and reaction times measured in a motor implicit learning task, were analyzed to detect changes in cortical excitability after 2-10 minutes of AC stimulation and sinusoidal DC stimulation (tSDCS) by using $1,10,15,30$, and $45 \mathrm{~Hz}$ and sham stimulation over the primary motor cortex in 50 healthy subjects (eight-16 subjects in each study).

\section{Results}

A significantly improved implicit motor learning was observed after $10 \mathrm{~Hz}$ AC stimulation only. No significant changes were observed in any of the analyzed frequency bands of EEG and with regard to the MEP amplitudes after AC or tSDCS stimulation. Similarly, if the anodal or cathodal DC stimulation was superimposed on 5, 10, and $15 \mathrm{~Hz}$ AC stimulation, the MEP amplitudes did not change significantly.

\section{Conclusions}

Transcranial application of weak AC current may appear to be a tool for basic and clinical research in diseases with altered EEG activity. However, its effect seems to be weaker than tDCS stimulation, at

This study was funded by the Bernstein Center for Computational Neuroscience (01GQ0432) (A.A.) and the Rose Foundation (C.P.).

Address reprint requests to: Dr Andrea Antal, Department of Clinical Neurophysiology, Georg-August University, Robert Koch Straße 40, 37075 Göttingen, Germany.

E-mail address: aantal@gwdg.de

Submitted August 9, 2007; revised October 12, 2007. Accepted for publication October 14, 2007. 
least in the present context of stimulus intensity and duration. Further studies are required to extend cautiously the safety range and uncover its influence on neuronal circuitries.

(c) 2008 Elsevier Inc. All rights reserved.

Keywords transcranial alternating current stimulation; transcranial magnetic stimulation; electroencephalogram; motor cortex; serial reaction time task

Neuroplasticity is an ongoing, self-organizing, adapting process that is widespread in cortical areas; it allows the brain to learn and adapt to new environmental situations. External influences on neuroplastic processes may be used for functional improvement of diseases, in particular for improving cortical functions such as learning or for studying brain function per se. Several methods exist to influence excitability of the brain by external means. The most wellknown is transcranial magnetic stimulation (TMS) introduced about 20 years ago. ${ }^{1}$ It followed transcranial electrical pulsed stimulation, which because of its painful stimulation characteristic never proceeded to a routine application method. ${ }^{2}$ Another electrical approach, weak direct current stimulation of the brain (transcranial direct current stimulation [tDCS]), was investigated intermittently within the last four decades, but entered into neurobiologic and clinical plasticity research ${ }^{3,4}$ only after its efficacy was unambiguously demonstrated by quantifying its effects during and after stimulation by single-pulse TMS over the motor cortex. ${ }^{5}$ TDCS is able to induce long-lasting changes in cortical excitability in different brain regions in a reversible, relatively selective, painless, and safe manner. Generally, motor cortex excitability is enhanced by anodal and decreased by cathodal stimulation, as seen in studies that used single-pulse TMS. Even though in humans the effects of tDCS were first demonstrated on the motor system, it also influences visual, somatosensory, and cognitive functions. ${ }^{6,7}$

Transcranial alternating current stimulation (tACS) of the brain is a new technique. It aims to interfere with ongoing oscillations in the brain. This technique may have important implications for neuropsychiatric disorders, for example, it has been concluded that measures of gamma synchrony offer a valuable window into the core integrative disturbance in schizophrenia. ${ }^{8}$ Recently, it was shown that inducing slow oscillation-like potential fields by transcranial application of oscillating potentials $(0.75 \mathrm{~Hz})$ during early nocturnal nonrapid eye-movement sleep, (a period of emerging slow wave sleep) enhances the retention of hippocampus-dependent declarative memory in healthy humans. ${ }^{9}$ The slowly oscillating potential stimulation induced an immediate increase in slow wave sleep, endogenous cortical slow oscillations, and slow spindle activity in the frontal cortex. Brain stimulation with oscillations at $5 \mathrm{~Hz}$-another frequency band that normally predominates during rapid eye-movement sleepdecreased slow oscillations and left declarative memory unchanged.
The aim of the current study is to further expand the stimulation spectrum between DC and AC stimulation. For this, we defined a frequency spectrum between 1 and $45 \mathrm{~Hz}$ transcranial electrical stimulation and analyzed motorevoked potentials (MEPs) and electroencephalogram (EEG) spectrum before and after AC stimulation, both with and without an anodal and cathodal DC shift. Intracellular and EEG recordings in animals ${ }^{10}$ have shown that modulating the excitability of cortical pyramidal cells generates a powerful and coherent feedback to the thalamus, resulting in highly coherent oscillations similar to those measured during natural sleep. These experiments are compatible with a role of the cortex in triggering and synchronizing oscillations generated in the thalamus, through cortico-thalamico-cortico loops, thus providing a possible cellular mechanism to explain the origin of large-scale coherent oscillations in the thalamocortical system. By stimulating the sensorimotor cortex with the use of tACS, oscillations can be triggered and may also reset ongoing rhythmic activity of local pacemaker networks consequently synchronizing brain oscillations.

Furthermore, behavioral tasks were used to study ACdriven changes in performance during a variant of the serial reaction time task (SRTT), ${ }^{11-13}$ which is a standard paradigm to test implicit motor learning. In this task, subjects perform finger movements repetitively without being aware of a sequential order. We applied tACS or sham stimulation to the primary motor cortex during performance of the task.

\section{Methods and materials}

\section{Subjects}

Fifty subjects ( 24 men and 26 women) participated in the studies. None of the subjects took regular or acute medication. Participants gave informed written consent. The experiments were approved by the Ethics Committee of the University of Göttingen, and conformed to the Declaration of Helsinki. All subjects were right handed, according to the Edinburgh handedness inventory. ${ }^{14}$

\section{Transcranial alternating current stimulation (tACS)}

Ten healthy subjects (22-43 years old, mean age $=26.4 \pm$ 8.0, 3 men) participated in the TMS study. Eight healthy subjects $(22-32$ years old, mean age $=25.75 \pm 3.28,3$ 
men) were involved in the EEG experiments. Two subjects participated in both the EEG and MEP experiments. Sixteen volunteers $(22-31$ years old, mean age $=22.4 \pm$ 4.15, 7 men) took part in the implicit learning study.

\section{Transcranial sinusoidal direct current stimulation (tSDCS)}

Ten healthy subjects (23-30 years old, mean age $=28.7 \pm$ 7.0, 6 men) were involved in the TMS study and 11 subjects took part in the EEG experiments (22-43 years old, mean age $=26.8 \pm 5.7,5$ men).

\section{tACS and tSDCS}

Electrical stimulation was delivered by a battery-driven constant-current stimulator (NeuroConn $\mathrm{GmbH}$, Ilmenau, Germany) through conductive-rubber electrodes, enclosed in two saline-soaked sponges. The stimulation electrode was placed over the left motor cortex, which was determined by single-pulse TMS. The reference electrode was placed over the contralateral orbit. The size of the stimulation electrode was $4 \times 4 \mathrm{~cm}$ and the reference electrode was $5 \times 10 \mathrm{~cm}$. The electrodes were fixed by elastic bands. tACS was applied for 5 minutes with a current intensity of $400 \mu \mathrm{A}$ and tSDCS for 2 or 4 minutes with a current intensity of $250 \mu \mathrm{A}$. Concerning tSDCS, the anodal or cathodal stimulation was sinusoidally modified. This kind of stimulation resulted in no change in polarity.

In the SRTT study, the current was delivered during the Blocks 2 to 5, which lasted approximately 7 minutes and in eight subjects during the Blocks 2 to 8 , which lasted approximately 10 minutes. The current was always ramped up or down over the first and last 2 seconds of stimulation. The maximal current density was $25 \mu \mathrm{A} / \mathrm{cm}^{2}$ in the case of tACS and $15.625 \mu \mathrm{A} / \mathrm{cm}^{2}$ in the tSDCS experiments over the motor cortex, which is below the safety parameters accepted for tDCS. ${ }^{15}$ The current density was 8 or $5 \mu \mathrm{A} / \mathrm{cm}^{2}$ with regard to the reference electrode. For sham stimulation, the current was turned on for 8 seconds at the beginning of the stimulation to achieve the light itching sensation under the electrode. However, it was more difficult to establish a sham condition with tACS because the rapid changes in current amplitude and direction caused flickering at the higher frequencies ( 30 and $45 \mathrm{~Hz}$ ) and experienced subjects might notice this sensation.

Subjects were blinded for stimulation conditions in all of the studies. In the case of tACS, the TMS study was double blind. However, as we mentioned previously, this statement with regard to the subjects was true only for the lower frequencies.

\section{Transcranial magnetic stimulation (TMS)}

TMS was performed by using a standard double ("figureof-eight") 70-mm coil connected to monophasic
Magstim200 stimulator (Magstim Company, Whiteland, Dyfed, UK). The coil was placed tangentially to the scalp, with the handle pointing posterolaterally at 45-degree angle from the midline. The optimum position was defined as the site where TMS resulted consistently in the largest MEP in the resting muscle. The site was marked with a waterproof skin marker to ensure that the coil was held in the correct position throughout the experimental sessions. Surface electromyography was recorded from the right first dorsal interosseus (FDI) muscle with the use of $\mathrm{Ag}-\mathrm{AgCl}$ electrodes in a belly-tendon montage. The signals were amplified and filtered (with a low-pass filter of $3 \mathrm{kHz}$, sampling rate of $5 \mathrm{kHz}$ ), digitized with a micro $1401 \mathrm{AD}$ converter (Cambridge Electronic Design, Cambridge, UK), recorded by a computer that used SIGNAL software (Cambridge Electronic Design, version 2.13). Data were analyzed offline. Complete muscle relaxation was controlled online via auditory and visual feedback of electromyography activity.

RMT was defined as the lowest stimulus intensity, which elicited a peak-to-peak MEP-amplitude of $50 \mu \mathrm{V}$, or more in the resting muscle, in at least 3 of 6 recordings. AMT was the minimum intensity eliciting a MEP of a superior size compared with moderate spontaneous muscular background activity (approximately 15\% of the maximum muscle strength) in at least 3 of 6 trials. ${ }^{16}$ The intensity of the stimulator output for the single test-pulse MEP was adjusted so that TMS led to an average MEP amplitude of about $1 \mathrm{mV}$ peakto-peak before electrical stimulation. The same stimulus intensity was used after the tSDC and tACS.

\section{EEG recording}

The EEG was recorded by using a 3-channel montage. One electrode was placed over $\mathrm{Oz}$ and 2 laterally above the motor region (C3 and $\mathrm{C} 4)$ in accordance with the international 10/20 system. The impedance was kept below $5 \mathrm{k} \Omega$. Linked mastoids (RLm) were used as references, the ground electrode was positioned on the forehead. Data were collected with a sampling rate of $1000 \mathrm{~Hz}$ with the use of the BrainAmp system (Brain Products $\mathrm{GmbH}$, Munich, Germany) and were analyzed offline (Brain Vision Analyzer, Brain Products $\mathrm{GmbH}$ ).

\section{Experimental design}

Subjects were seated in a comfortable reclining chair with a mounted headrest throughout the experiments. Within each type of experimental technique, the MEP-EEG measurements were always performed by the same investigator.

\section{TMS study}

\section{tACS}

Ten subjects participated in 6 experimental sessions on separate days, 1 day apart to avoid carry over effects. The 
TMS experiments were performed at identical times. The subjects received $1,10,15,30$, and $45 \mathrm{~Hz}$ tACS and sham stimulation in a randomized order. RMT, AMT, the intensity to evoke MEP of approximately $1 \mathrm{mV}$ peak-to-peak amplitude, single-test pulse MEPs were recorded before tACS. Stimulus intensities (in percentage of maximal stimulator output) of TMS were determined at the beginning of each experiment. Thirty single-test pulse MEPs were recorded 7 times after the stimulation, ie, approximately 0 minutes after tACS, 2, 4, 7, 10, 15, and 20 minutes after the end of $\mathrm{AC}$ stimulation.

\section{tSDCS}

Ten subjects received anodal and 7 received cathodal tSDCS with a frequency 5,10, and $15 \mathrm{~Hz}$ for 2 minutes in a counterbalanced order. Stimulations were performed on separate days and between each stimulation session was a 15-minute break. Fifty single-test pulse MEPs were recorded before and 40 MEPs after tSDCS (averaged in 20 blocks).

\section{EEG study}

\section{tACS}

The EEG experiments were conducted in a repeated measurement design using a randomized order, with a break of minimum 20 minutes between each stimulation session. A 2-minute EEG was recorded at rest before and 3 times after AC stimulation (immediately, 7, and 14 minutes after the end of the stimulation). Subjects received 1, 10, and $45 \mathrm{~Hz}$ tACS in a randomized and counterbalanced order.

\section{tSDCS}

The tSDCS was administered at 5,10 , and $15 \mathrm{~Hz}$ in a randomized order, with a 20-minute break between stimulation sessions. A 2-minute EEG was recorded before stimulation, and then a 4-minute EEG recorded immediately after stimulation. Subjects received tSDC for a 4minute duration at an intensity of $250 \mu \mathrm{A}$ in both an anodal and cathodal direction.

\section{SRTT}

Subjects were seated in front of a computer screen at eye level behind a response pad with 4 buttons numbered 1 to 4 , and were instructed to push each button with a different finger of the right hand (index finger for button 1, middle finger for button 2, ring finger for button 3 , and little finger for button 4). An asterisk appeared in 1 of 4 positions horizontally spaced on a computer screen and permanently marked by dots. The subjects were instructed to press the key corresponding to the position of the asterisk as fast as possible. After a button was pushed, the go signal disappeared. The next go signal was displayed 500 milliseconds later. The test consisted of 8 blocks of 120 trials. In
Blocks 1 and 6 , the sequence of asterisks followed a pseudorandom order in which asterisks were presented equally frequently in each position and never in the same position in 2 subsequent trials. In Blocks 2 to 5 and 7 and 8, the same 12-trial sequence of asterisk positions repeated itself 10 times (abadbcdacbdc). Subjects were not told about the repeating sequence.

Where improved performance during the whole course of the task is due to implicit learning as well as to increasing task routine, differences in performance between Block 5 and the random Block 6 represent a measure of implicit learning only, because task routine is thought to be equivalent in both blocks, and thus any differences in performance should be due to implicit sequence learning. ${ }^{17}$

In 6 subjects, the first 3 blocks of the previously used test was repeated 1 (Block 9: pseudorandom; Blocks 10 and 11 repeated sequences) and 2 hours (Block 12: pseudorandom; Blocks 13 and 14: repeated sequences) poststimulation. Differences in performance between Blocks 9 and 10 and 12 and 13 represent a measure of implicit learning.

\section{Safety aspects}

All the subjects completed a questionnaire the next day after the completion of the experimental sessions. The questionnaire contained rating scales for the presence and severity of headache, difficulties in concentrating, acute mood changes, visual perceptual changes, fatigue, and discomforting sensations like pain, tingling, itching, or burning under the electrodes during and after tACS. ${ }^{18}$

\section{Analysis of the data}

\section{EEG analysis}

EEG epochs (2 minutes) were segmented for 30 seconds and filtered by using $0.1 \mathrm{~Hz}$ ( $24 \mathrm{~dB} /$ octave) low cutoff and a $70 \mathrm{~Hz}(24 \mathrm{~dB} /$ octave) high cutoff and $50 \mathrm{~Hz}$ notch filters. In addition to semiautomatic artifact detection (200 $\mu \mathrm{V}$ amplitude criterion), all epochs were visually inspected, and those containing eye blinks or muscle movement artifacts were excluded. After artifact rejection all of the epochs were segmented into 2 seconds, and fast fourier transformation (FFT) was calculated for all electrodes $(0.5$ $\mathrm{Hz}$ resolution, and 10\% Hamming-window). The FFT segments were averaged for each 30 seconds. The mean activity in voltage was calculated and exported from each frequency bands (theta band $4.5-7 \mathrm{~Hz}$, alpha band $8-12 \mathrm{~Hz}$, beta band $12.5-30 \mathrm{~Hz}$, and gamma band $31-49 \mathrm{~Hz}$ ) for statistical analysis.

\section{MEP analysis}

Peak-to-peak amplitudes (mV) of each MEP were measured offline, and mean MEP amplitudes were calculated for each stimulation condition for each time point separately. The amplitudes were normalized to baseline. 


\section{SRTT analysis}

In each trial, response time (RT) was measured from the appearance of the go signal until the first button was pushed by the subject. For each block of trials of a given experimental condition, mean RT was calculated for each subject separately. Furthermore, the SD of RTs for each subject in every block was calculated as an index of variability of RTs. An error rate (ER) was calculated to assess the number of incorrect responses for each block and each subject in each stimulation condition.

\section{Statistical analysis}

In all of the EEG and TMS experiments a repeated measures of analysis of variance (ANOVA) (a given current condition versus sham $\times$ time points of poststimulation; dependent variable: mean amplitude of MEPs, FFT power in a given frequency band) was calculated. In case of the significant interaction of time and stimulation condition, a post hoc test was performed.

Concerning the implicit learning statistical analyses were performed with repeated measures of ANOVA (current conditions $\times 8$ blocks) for RT, ER, and variability. Because the RT and ER differences between Blocks 5 and 6 are thought to represent an exclusive measure of implicit learning, interactive Student $t$ tests $^{19}$ were performed to compare the respective differences for the alternating current stimulation condition versus sham condition.

\section{Results}

All the subjects tolerated the stimulation; none of the experimental sessions were interrupted because of side effects of the stimulation. However, about half of the subjects noticed light flickering during higher frequency stimulation $(30,45 \mathrm{~Hz})$ by using an intensity of $0.4 \mathrm{~mA}$. As a result, we did not further increase the stimulation amplitude for safety reasons. Only 2 of the subjects reported a light burning sensation under the electrodes during the stimulation. Six subjects experienced a mild headache that lasted for a maximum of 2 hours after the end of tACS, independent from the frequency of the stimulation. None of the subjects reported transient side effect according to the 1 to 2 weeks' follow-up.
MEP

\section{tACS}

Repeated measurements of ANOVA $(1,10,15,30,45 \mathrm{~Hz}$ versus sham stimulation condition separately $\times 7$ time points poststimulation) revealed no significant interactions between current condition and time in any of the comparisons between tACS and sham stimulation. For $1-\mathrm{Hz}$ stimulation, there was no main effect of stimulation $[\mathrm{F}(1,18)=0.03, P=.86]$ and time course $[\mathrm{F}(6,108)=$ $1.49, P=.19]$. The interaction between stimulation und time was also not significant $[\mathrm{F}(6,108)=1.17, P=.32]$. For $10-\mathrm{Hz}$ stimulation, there was no main effect of stimulation $[\mathrm{F}(1,18)=3.2, P=.09]$ and time course $[\mathrm{F}(6,108)=$ $1.0, P=.42]$. The interaction between stimulation and time was also not significant $[\mathrm{F}(6,108)=0.61, P=.72]$. For 15$\mathrm{Hz}$ stimulation, there was no main effect of stimulation $[\mathrm{F}(1,18)=0.004, P=.95]$ and time course $[\mathrm{F}(6,108)=$ $0.62, P=.71]$. The interaction between stimulation and time was also not significant $[\mathrm{F}(6,108)=1.77, P=.11]$. For $30-\mathrm{Hz}$ stimulation, there was no main effect of stimulation $[\mathrm{F}(1,18)=0.14, P=.71]$ and time course $[\mathrm{F}(6,108)=$ $0.05, P=.99]$. The interaction between stimulation and time was also not significant $[\mathrm{F}(6,108)=1.03, P=.4]$. For $45-\mathrm{Hz}$ stimulation, there was no main effect of stimulation $[\mathrm{F}(1,18)=0.01, P=.91]$ and time course $[\mathrm{F}(6,108)=$ $0.71, P=.64]$. The interaction between stimulation and time was also not significant $[\mathrm{F}(6,108)=0.4, P=.87]$. Table 1 shows the mean MEP values and their standard error before and after tACS.

\section{tSDCS}

Here, anodal or cathodal stimulation was sinusoidally modified at a given frequency. The ANOVA was calculated separately for anodal and cathodal stimulation (5-, 10-, and $15-\mathrm{Hz}$ stimulation conditions and 2 time points poststimulation). The analysis revealed no significant interactions between current condition and time in either the anodal or cathodal condition ( $\mathrm{F}<1.2, P>.3)$. An increase in motorcortical excitability, after the combination of anodal and $15-\mathrm{Hz}$ stimulation, of approximately $35 \%$ was observed after stimulation, but was not significant when compared with the baseline values $(P=.08)$. Table 2 shows the mean MEP values and their standard error before and after tSDCS.

Table 1 Mean MEP amplitudes (SEM) before and after tACS at 1-, 5-, 10-, 15-, and 30-Hz stimulation

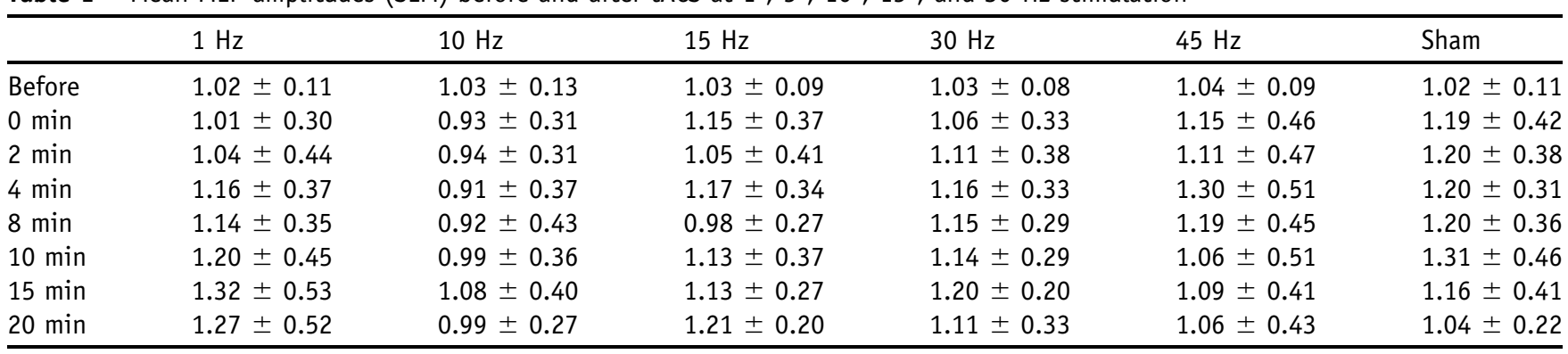

A decrease of the MEP amplitude after $10-\mathrm{Hz}$ stimulation was observed, but was not significant. 
Table 2 Mean MEP amplitudes (SEM) before and after tSDCS at 5-, 10- and 15- Hz stimulation

\begin{tabular}{|c|c|c|c|c|c|c|}
\hline & \multicolumn{3}{|c|}{ Anodal (mean MEPs and SEM) } & \multicolumn{3}{|c|}{ Cathodal (mean MEPs and SEM) } \\
\hline & Before & 2 min after & 4 min after & Before & 2 min after & 4 min after \\
\hline $5 \mathrm{~Hz}$ & $1.12 \pm 0.1$ & $1.12 \pm 0.2$ & $1.16 \pm 0.2$ & $0.92 \pm 0.06$ & $1.08 \pm 0.14$ & $0.8 \pm 0.15$ \\
\hline $15 \mathrm{~Hz}$ & $1.2 \pm 0.03$ & $1.6 \pm 0.2$ & $1.37 \pm 0.11$ & $0.89 \pm 0.1$ & $1.08 \pm 0.2$ & $0.9 \pm 0.2$ \\
\hline
\end{tabular}

An increase of the MEP amplitude after anodal $15 \mathrm{~Hz}$ stimulation was observed, but was not significant.

\section{EEG}

\section{tACS}

The ANOVA (1-, 10-, and 45-Hz stimulation conditions $\times$ 16 time points - including baseline $\times 3$ channels) was calculated separately for each frequency band. It revealed no significant interactions among current conditions, time, and channels at any of the different frequencies applied. The results of the statistical analysis are summarized in Table 3.

\section{tSDCS}

The ANOVA was calculated for anodal and cathodal directions and for each frequency band separately. The analyses (5-, 10-, and $15-\mathrm{Hz}$ stimulation conditions $\times 16$ time points - including baseline $\times 3$ channels) revealed no significant interactions between current conditions and time at any of the different frequencies applied $(\mathrm{F}<0.5, P>.4)$. The results of the statistical analysis are summarized in Table 4.

\section{SRTT}

RTs of the SRTT shortened during tACS of the primary motor cortex; repeated measures ANOVA (1-, 10-, 45-Hz, and sham stimulation conditions $\times 8$ blocks) revealed a significant effect on blocks $[\mathrm{F}(7,105)=33.11 ; \mathrm{P}<.001]$. This was caused by an interaction of alternating current versus sham stimulation for Block 5 and Block 6 , caused by a greater difference in the alternating current stimulation in the case of $10-\mathrm{Hz}$ stimulation $(t=-3.26, d f=15, P=.005)$ as revealed by Student $t$ tests. Figure 1 shows the differences between $10-\mathrm{Hz}$ and sham stimulation. Despite the significant main effect of blocks in ANOVA, the results of all other tests remained insignificant. However, a trend toward reduced RTs in Blocks 2 to 5 and 7 and 8 for tACS compared with the sham condition was identified.

The paradigm was repeated in 6 subjects after 1 and 2 hours poststimulation. At these time points the RTs were not different between the $10-\mathrm{Hz}$ and sham stimulation conditions (Fig 1).

To exclude the possibility that tACS speeds up the initiation of movements and does not modify implicit learning, the $10-\mathrm{Hz}$ and sham stimulations were repeated during Block 2 to 8 in 8 subjects by using different sequences than before. During the random block (Block 6) tACS and sham stimulation did not have a differential effect on RTs $(P=.64)$. However, the ratio of Block 5 and Block 6 , because of a greater difference in the $10-\mathrm{Hz}$ stimulation condition remained significant $(t=-2.41, d f=7$, $P=.046)$.

For ER, the ANOVAs showed a significant main effect on current $(P<.001)$ and blocks at $1 \mathrm{~Hz}(P=.012)$ and at $45 \mathrm{~Hz}(P=.001)$, there was no significant condition $X$ blocks interaction. Student $t$ tests revealed no significant

Table 3 The results of the repeated measures ANOVA of the EEG experiment with tACS

\begin{tabular}{|c|c|c|c|}
\hline & $d f$ & $F$ value & $P$ value \\
\hline \multicolumn{4}{|l|}{ Theta band } \\
\hline Stim & 2 & 0.037 & .964 \\
\hline Channel & 2 & 82.709 & $<.001^{*}$ \\
\hline Time & 15 & 1.843 & $.028^{*}$ \\
\hline Stim $\times$ Channel & 4 & 0.130 & .971 \\
\hline Stim $\times$ Time & 30 & 1.021 & .440 \\
\hline Channel $\times$ Time & 30 & 1.010 & .453 \\
\hline Stim $\times$ Channel $\times$ Time & 60 & 1.198 & .154 \\
\hline \multicolumn{4}{|l|}{ Alpha band } \\
\hline Stim & 2 & 0.002 & .998 \\
\hline Channel & 2 & 13.614 & $<.001^{*}$ \\
\hline Time & 15 & 6.343 & $<.001^{*}$ \\
\hline Stim $\times$ Channel & 4 & 0.053 & .995 \\
\hline Stim $\times$ Time & 30 & 0.674 & .904 \\
\hline Channel $\times$ Time & 30 & 1.125 & .298 \\
\hline Stim $\times$ Channel $\times$ Time & 60 & 0.837 & .803 \\
\hline \multicolumn{4}{|l|}{ Beta band } \\
\hline Stim & 2 & 0.089 & .916 \\
\hline Channel & 2 & 14.952 & $<.001^{*}$ \\
\hline Time & 15 & 1.102 & .353 \\
\hline Stim $\times$ Channel & 4 & 0.054 & .994 \\
\hline Stim $\times$ Time & 30 & 0.774 & .799 \\
\hline Channel $\times$ Time & 30 & 1.017 & .442 \\
\hline Stim $\times$ Channel $\times$ Time & 60 & 0.648 & .981 \\
\hline \multicolumn{4}{|l|}{ Gamma band } \\
\hline Stim & 2 & 0.106 & .900 \\
\hline Channel & 2 & 0.097 & .908 \\
\hline Time & 15 & 0.723 & .761 \\
\hline Stim $\times$ Channel & 4 & 0.193 & .941 \\
\hline Stim $\times$ Time & 30 & 0.572 & .967 \\
\hline Channel $\times$ Time & 30 & 1.590 & $.025^{*}$ \\
\hline Stim $\times$ Channel $\times$ Time & 60 & 0.771 & .896 \\
\hline
\end{tabular}

Independent variables: time course (Time), condition of current stimulation (Stim), and EEG channels (Channel); dependent variable: FFT power in a given frequency band. The ANOVA revealed no significant interactions between current conditions, time, and channels at any of the different frequencies applied. The asterisk indicates significant $P$-values $(P<.05)$. 
Table 4 The results of the repeated measures ANOVA of the EEG experiment with tSDCS

\begin{tabular}{|c|c|c|c|c|c|c|}
\hline & \multicolumn{3}{|c|}{ Anodal } & \multicolumn{3}{|c|}{ Cathodal } \\
\hline & $d f$ & $F$ value & $P$ value & $d f$ & $\mathrm{~F}$ value & $P$ value \\
\hline \multicolumn{7}{|l|}{ Theta band } \\
\hline Stim & 2 & 1.085 & .351 & 2 & 0.194 & .824 \\
\hline Channel & 2 & 0.511 & .602 & 2 & 3.149 & $.05^{*}$ \\
\hline Time & 7 & 1.191 & .309 & 7 & 2.821 & $.007^{*}$ \\
\hline Stim $\times$ Channel & 4 & 0.901 & .469 & 4 & 0.706 & .591 \\
\hline Stim $\times$ Time & 14 & 0.469 & .947 & 14 & 1.121 & .34 \\
\hline Channel $\times$ Time & 14 & 0.857 & .606 & 14 & 0.92 & .536 \\
\hline Stim $\times$ Channel $\times$ Time & 28 & 1.143 & .283 & 28 & 1.157 & .268 \\
\hline \multicolumn{7}{|l|}{ Alpha band } \\
\hline Stim & 2 & 0.011 & .989 & 2 & 0.861 & .433 \\
\hline Channel & 2 & 1.026 & .365 & 2 & 0.926 & .401 \\
\hline Time & 7 & 1.062 & .388 & 7 & 1.734 & .102 \\
\hline Stim $\times$ Channel & 4 & 0.492 & .742 & 4 & 0.952 & .44 \\
\hline Stim $\times$ Time & 14 & 0.464 & .949 & 14 & 0.717 & .755 \\
\hline Channel $\times$ Time & 14 & 1.456 & .124 & 14 & 0.579 & .881 \\
\hline Stim $\times$ Channel $\times$ Time & 28 & 1.169 & .255 & 28 & 1.348 & .113 \\
\hline \multicolumn{7}{|l|}{ Beta band } \\
\hline Stim & 2 & 0.092 & .912 & 2 & 1.076 & .353 \\
\hline Channel & 2 & 2.292 & .109 & 2 & 1.784 & .176 \\
\hline Time & 7 & 2.94 & .005 & 7 & 0.713 & .66 \\
\hline Stim $\times$ Channel & 4 & 0.987 & .422 & 4 & 0.201 & .936 \\
\hline Stim $\times$ Time & 14 & 0.404 & .972 & 14 & 0.888 & .572 \\
\hline Channel $\times$ Time & 14 & 1.016 & .436 & 14 & 1.426 & .136 \\
\hline Stim $\times$ Channel $\times$ Time & 28 & 0.901 & .614 & 28 & 1.038 & .413 \\
\hline \multicolumn{7}{|l|}{ Gamma band } \\
\hline Stim & 2 & 0.135 & .874 & 2 & 0.51 & .605 \\
\hline Channel & 2 & 0.258 & .772 & 2 & 1.559 & .218 \\
\hline Time & 7 & 2.286 & .028 & 7 & 0.735 & .641 \\
\hline Stim $\times$ Channel & 4 & 0.444 & .776 & 4 & 0.37 & .828 \\
\hline Stim $\times$ Time & 14 & 0.377 & .98 & 14 & 0.806 & .661 \\
\hline Channel $\times$ Time & 14 & 0.659 & .813 & 14 & 3.19 & $.001^{*}$ \\
\hline Stim $\times$ Channel $\times$ Time & 28 & 0.762 & .804 & 28 & 0.689 & .884 \\
\hline
\end{tabular}

Independent variables: time course (Time), condition of current stimulation (Stim) and EEG channels (Channel); dependent variable: FFT power in a given frequency band. The ANOVA revealed no significant interactions between current conditions, time, and channels at any of the different frequencies applied. The asterisk indicates significant $P$-values $(P<.05)$.

difference between Block 5 and Block 6. For variability, the ANOVAs showed a significant main effect of condition $(P<.001)$ and blocks $(P<.001)$ without significant interaction between condition and blocks at all frequencies. Student $t$ tests revealed no significant difference between Blocks 5 and 6.

\section{Comment}

The main result of the current study is that $10-\mathrm{Hz}$ tACS over the motor cortex by using 7-minute stimulation duration was able to improve implicit motor learning. However, $10 \mathrm{~Hz}$ AC stimulation did not modify the EEG power and the MEP amplitudes significantly, when compared with sham stimulation.

In our study, only $10 \mathrm{~Hz}$ of tACS significantly improved performance in the acquisition and early consolidation phase of implicit motor learning. Compared with the noncurrent stimulation condition, reaction times in the SRTT decreased significantly and became faster during the course of the experiment. This result is similar to the effect of anodal stimulation over the M1 reported by a previous study. ${ }^{20}$ Previous studies suggest that an excitability enhancement seems to be a necessary condition for learning by inducing strengthening of synapses/long-termpotentiation by modifying NMDA-receptor efficacy. ${ }^{21,22}$ Regarding studies in the human, this is in line with observations of increased activation of the motor cortex during motor learning tasks, ${ }^{23,24}$ and also with pharmacologic results showing that results of motor training can be improved by cortical excitability enhancements. ${ }^{25}$ It appears that a $10-\mathrm{Hz}$ tACS-driven cortical excitability change could facilitate the learning process.

The nonsignificant MEP changes were probably caused by the low number of subjects, larger sample sizes would have been necessary to prove the significant effect of 


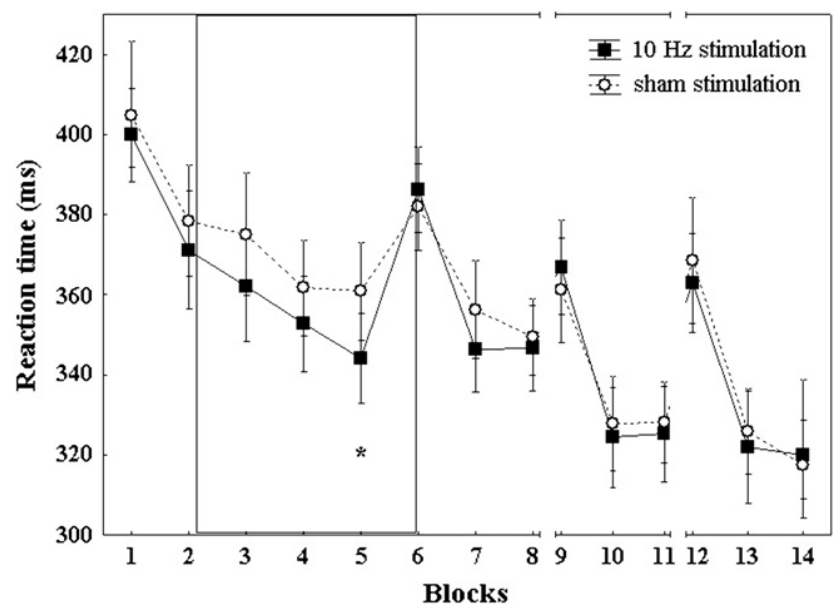

Figure 1 10-Hz tACS of the primary motor cortex improves implicit motor learning in its early phase. Reaction times decrease faster in the $10 \mathrm{~Hz}$ stimulation condition when compared with the sham-stimulation condition. Moreover, the RT difference comparing Blocks 5 and 6, which indicates implicit sequence learning, is larger for the $10-\mathrm{Hz}$ stimulation condition, when compared with the nonstimulation condition. The asterisk indicates a significant difference regarding reaction time differences between Blocks 5 and 6 , and between $10-\mathrm{Hz}$ and sham stimulation. In 1 and 2 hours poststimulation, this significant difference was not detectable any more.

stimulation if there is any. However, we also noticed that the SDs of the MEP amplitudes were higher when compared with those measured by previous studies that used tDCS. $^{3,4}$ Similar to our results, it was also published that there was no significant change in corticospinal excitability after $10-\mathrm{Hz}$ rTMS. $^{26}$ Nevetheless, in our study $10 \mathrm{~Hz}$ of tACS had a facilitatory effect on motor performance. The only difference between the 2 tACS studies was the duration of the stimulation: in the TMS study, a shorter stimulation duration was applied than that used in the implicit learning study. Therefore, it may be possible that the effect of $10 \mathrm{~Hz}$ tACS is stimulation duration-dependent: a shorter stimulation duration may not be strong enough to modify cortical excitability, whereas a longer stimulation duration may have facilitatory effects.

We used a relatively small stimulation electrode size in order to enhance the focality of stimulation ${ }^{27}$ and larger reference electrode to avoid the stimulation of the frontopolar cortex and retina. However, half of the subjects still noticed a flickering in their visual field, mainly during highfrequency stimulation. This means that at 30 and $45 \mathrm{~Hz}$ stimulation conditions in our study was not precisely "double-blind" per se because the subjects could feel the difference between sham and verum stimulation. This problem with sham stimulation was not encountered with tDCS. ${ }^{18}$ Further increase of the reference electrode size might help but technically is not possible therefore, in the future, systematically exploring the effect of electrical stimulation with the use of new electrode positions (eg, M1-occipital cortex or extracephalic reference) is necessary. If intensities are comparable between tDCS and tACS $0.4 \mathrm{~mA}$ might be at the lower border for inducing after effects. ${ }^{3}$ Thus, it remains to be seen if higher intensities or longer stimulation durations are better for inducing after effects, notwithstanding the assumption that higher stimulation intensities could be potentially more dangerous with respect to seizure induction. Along this suspicion, a similar new method, called focal electrically administered therapy (FEAT), uses also AC current. FEAT can be boosted to induce focal seizures, then referred to as "focal electrically administered seizure therapy" (FEAST). ${ }^{28}$ This form of AC stimulation is intended to be used for a more focal form of electroconvulsive therapy in the future.

In conclusion, the transcranial application of weak $\mathrm{AC}$ current may appear to be a promising tool for clinical neuroplasticity research, for it allows a painless, selective, focal, noninvasive, and reversible excitability modulation of the cortex. Important research still has to be performed, mainly in uncovering the mode of function and in finding a way to prolong the effects of weak current application further, as has already been successfully done in DC research.

\section{References}

1. Barker AT, Jalinous R, Freeston IL. Non-invasive magnetic stimulation of human motor cortex. Lancet 1985;1:1106-1107.

2. Merton PA, Morton HB. Stimulation of the cerebral cortex in the intact human subject. Nature 1980;285:227.

3. Nitsche MA, Paulus W. Excitability changes induced in the human motor cortex by weak transcranial direct current stimulation. J Physiol 2000;527(Pt 3):633-639.

4. Nitsche MA, Paulus W. Sustained excitability elevations induced by transcranial DC motor cortex stimulation in humans. Neurology 2001;57:1899-1901.

5. Priori A, Berardelli A, Rona S, Accomero N, Manfredi M. Polarization of the human motor cortex through the scalp. Neuroreport 1998;9:2257-2260.

6. Antal A, Nitsche MA, Paulus W. Transcranial direct current stimulation and the visual cortex. Brain Res Bull 2006;68:459-463.

7. Fregni F, Pascual-Leone A. Technology insight: noninvasive brain stimulation in neurology-perspectives on the therapeutic potential of rTMS and tDCS. Nat Clin Pract Neurol 2007;3:383-393.

8. Lee KH, Williams LM, Breakspear M, Gordon E. Synchronous gamma activity: a review and contribution to an integrative neuroscience model of schizophrenia. Brain Res Brain Res Rev 2003;41:57-78.

9. Marshall L, Helgadottir H, Molle M, Born J. Boosting slow oscillations during sleep potentiates memory. Nature 2006;444:610-613.

10. Destexhe A, Contreras D, Steriade M. Spatiotemporal analysis of local field potentials and unit discharges in cat cerebral cortex during natural wake and sleep states. J Neurosci 1999;19:4595-4608.

11. Nissen MJ, Bullemer P. Attentional requirements of learning: Evidence from performance measures. Cognit Psychol 1987;19:1-32.

12. Exner C, Koschack J, Irle E. The differential role of premotor frontal cortex and basal ganglia in motor sequence learning: evidence from focal basal ganglia lesions. Learn Mem 2002;9:376-386.

13. Nitsche MA, Schauenburg A, Lang N, et al. Facilitation of implicit motor learning by weak transcranial direct current stimulation of the primary motor cortex in the human. J Cognit Neurosci 2003;15: 619-626. 
14. Oldfield RC. The assessment and analysis of handedness: the Edinburgh inventory. Neuropsychologia 1971;9:97-113.

15. Nitsche MA, Liebetanz D, Lang N, et al. Safety criteria for transcranial direct current stimulation (tDCS) in humans. Clin Neurophysiol 2003;114:2220-2222.

16. Rothwell JC, Hallett M, Berardelli A, et al. Magnetic stimulation: motor evoked potentials: the International Federation of Clinical Neurophysiology. Electroencephalogr Clin Neurophysiol Suppl 1999;52: 97-103.

17. Pascual-Leone A, Grafman J, Hallett M. Modulation of cortical motor output maps during development of implicit and explicit knowledge. Science 1994;263:1287-1289.

18. Poreisz C, Boros K, Antal A, Paulus W. Safety aspects of transcranial direct current stimulation concerning healthy subjects and patients. Brain Res Bull 2007;72:208-214.

19. Cohen J. Statistical power analysis for the social sciences. 2nd ed. New York: Academic Press, New York, 1977.

20. Nitsche MA, Liebetanz D, Antal A, et al. Modulation of cortical excitability by weak direct current stimulation-technical, safety and functional aspects. Suppl Clin Neurophysiol 2003;56:255-276.
21. Bennett MR. The concept of long term potentiation of transmission at synapses. Prog Neurobiol 2000;60:109-137.

22. Rioult-Pedotti MS, Friedman D, Hess G, Donoghue JP. Learninginduced LTP in neocortex. Science 2000;290:533-536.

23. Grafton ST, Mazziotta JC, Presty S, et al. Functional anatomy of human procedural learning determined with regional cerebral blood flow and PET. J Neurosci 1992;12:2542-2548.

24. Honda M, Deibner MP, Ibanez V, et al. Dynamic cortical involvement in implicit and explicit motor sequence learning: a PET study. Brain 1998;121(Pt11):2159-2173

25. Butefisch CM, Davis BC, Sawaki L, et al. Modulation of use-dependent plasticity by d-amphetamine. Ann Neurol 2002;51:59-68.

26. Rossi S, Rossini PM. TMS in cognitive plasticity and the potential for rehabilitation. Trends Cogn Sci 2004;8:273-279.

27. Nitsche MA, Doemkes S, Karakose T, et al. Shaping the effects of transcranial direct current stimulation of the human motor cortex. J Neurophysiol 2007;97:3109-3117.

28. George MS, Nahas Z, Borckardt JJ, et al. Brain stimulation for the treatment of psychiatric disorders. Curr Opin Psychiatry 2007;20: 250-254. 


\subsection{Brain-derived neurotrophic factor (BDNF) gene polymorphisms shape cortical plasticity in humans}

Antal A, Chaieb L, Moliadze V, Monte- Silva K, Poreisz C, Thirugnanasambandam N, Nitsche M, Shoukier M, Ludwig H, Paulus W. Brain Stimulation 2010;

Recent studies suggest that the expression of the brain- derived neurotrophic factor (BDNF) gene may influence mechanisms of synaptic plasticity in the human cortex and that they, in turn, may influence the efficacy of applied external electric and magnetic stimulation techniques (Cheeran et al., 2008, Yoshii and Constantine-Paton, 2010). Here we have retrospectively explored the interaction of the BDNF polymorphism with the currently used methods of external electric and magnetic stimulation: tDCS, tRNS, tACS and a patterned form of rTMS (repetitive TMS) termed iTBS (intermittent theta burst stimulation). The data of 15 healthy subjects who possessed the Val66Met allele, 46 subjects with the homozygous Val66Val allele, and 3 with the Met66Met polymorphism was analysed for either enhanced or reduced neuroplastic aftereffects. For iTBS, plastic aftereffects could only be induced in carriers of the Val66Val allele; for anodal and cathodal tDCS carriers of the Val66Met allele showed enhanced plasticity, whereas there was no significant difference in the response of the groups to tRNS. His study demonstrates that the BDNF polymorphism does indeed shape mechanisms of plasticity in the human brain and suggests that future studies should take into account the interaction between stimulation and genetic background when optimizing stimulation parameters for therapeutic purposes. 


\title{
Brain-derived neurotrophic factor (BDNF) gene polymorphisms shape cortical plasticity in humans
}

\author{
Andrea Antal ${ }^{a}$, Leila Chaieb ${ }^{a}$, Vera Moliadze ${ }^{a}$, Katia Monte-Silva ${ }^{a}$, \\ Csaba Poreisz $^{a}$, Nivethida Thirugnanasambandam ${ }^{a}$, Michael A. Nitsche ${ }^{a}$, \\ Moneef Shoukier ${ }^{b}$, Harald Ludwig ${ }^{b}$, Walter Paulus, ${ }^{a}$
}

\author{
${ }^{a}$ Department of Clinical Neurophysiology, Georg-August University of Göttingen, Göttingen, Germany \\ ${ }^{b}$ Institute of Human Genetics, Georg-August University of Göttingen, Göttingen, Germany
}

\begin{abstract}
Background
The brain-derived neurotrophic factor $(B D N F)$ gene is involved in mechanisms of synaptic plasticity in the adult brain. It has been demonstrated that $B D N F$ also plays a significant role in shaping externally induced human brain plasticity. Plasticity induced in the human motor cortex by intermittent thetaburst stimulation (iTBS) was impaired in individuals expressing the Val66Met polymorphism.
\end{abstract}

\section{Methods}

To explore whether this polymorphism is also important for other neuroplasticity-inducing tools in humans with modes of action differing from that of iTBS, namely, transcranial direct current (tDCS) and random noise stimulation (tRNS), we retrospectively analyzed the data of 64 subjects studied in our laboratory with regard to $B D N F$ genotype.

\section{Results}

Fifteen subjects with the Val66Met allele, 46 subjects with the Val66Val allele, and 3 Met66Met carriers were identified. The response of the Val66Met allele carriers to stimulation differed in two protocols compared with the response of Val66Val individuals. For iTBS (15 subjects, 5 heterozygotes), plasticity could be only induced in the Val66Val allele carriers. However, for facilitatory tDCS (24 subjects, 10 heterozygotes), as well as for inhibitory tDCS, (19 subjects, 8 heterozygotes), carriers of the Val66Met allele displayed enhanced plasticity, whereas for transcranial random noise stimulation (29 subjects, 8 heterozygotes), the difference between groups was not so pronounced.

\section{Conclusions}

$B D N F$ polymorphism has a definite impact on plasticity in humans, which might differ according to the mechanism of plasticity induction. This impact of $B D N F$ on plasticity should be taken into account for

This study was initiated and funded by an unrestricted grant awarded by the Rose Foundation (L.C./W.P.) and by the Bernstein Center for Computational Neuroscience Göttingen (V.M./W.P.) (BMBF 01GQ0782).

Correspondence: Andrea Antal, PhD, Department of Clinical Neurophysiology, Georg-August University, Robert-Koch-Str. 40, 37075 Göttingen, Germany.

E-mail address: Aantal@gwdg.de

Submitted October 19, 2009; revised December 8, 2009. Accepted for publication December 16, 2009. 
future studies, as well as having wider ranging implications for the treatment of neuropsychiatric disorders with transcranial stimulation tools, as it may predetermine their efficacy for the treatment of disease and rehabilitation.

(C) 2010 Elsevier Inc. All rights reserved.

Keywords transcranial random noise stimulation; transcranial direct current stimulation; transcranial magnetic stimulation; neuroplasticity; $B D N F$

Brain-derived neurotrophic factor $(B D N F)$ regulates cell survival, proliferation, and synaptic growth in the central nervous system. In the adult brain, it has been shown to modulate N-methyl-D-aspartate (NMDA) receptordependent long-term potentiation (LTP) ${ }^{1}$ and long-term depression (LTD). ${ }^{2}$ Specifically, mature (m)BDNF has been found to play a role in all stages of $\mathrm{LTP}^{3}$ whereas the precursor peptide (pro- $B D N F$ ) is associated with LTD. $B D N F$ is present with a frequent polymorphism resulting from a nonconservative amino acid substitution (valine [Val] to methionine [Met]) at codon 66 (30\% Val66Met in the caucasian population). It has recently been shown that this polymorphism dramatically alters the intracellular trafficking and packaging of pro- $B D N F$ in rats and, thus the regulated secretion of the mature peptide. ${ }^{4}$ In humans, the $B D N F$ Val66Met polymorphism is known to have functional consequences in healthy subjects, including reduced recall capacity with regard to episodic memory. ${ }^{4,5}$

Recently, the noninvasive induction of neuroplastic processes by transcranial stimulation techniques has been frequently implemented for the functional improvement of diseases and for enhancing cortical functions. The most well-known method currently used to influence motor cortex (M1) excitability of the brain is repetitive transcranial magnetic stimulation (rTMS) ${ }^{6}$; extended to the more efficient variant termed "theta burst stimulation" (TBS), which is a patterned rTMS paradigm. ${ }^{7}$ Memantine can block the facilitatory after effect of intermittent TBS (iTBS) suggesting that the effects of iTBS rely on NMDA-receptor potentiation. ${ }^{8}$ A different approach to inducing global/nonfocal plasticity is based on a modulatory technique: transcranial direct current stimulation (tDCS). The tDCS represents the other end of the spectrum; by delivering subthreshold stimuli in the form of a continuous electric current, it polarizes neuronal membranes and alters the spontaneous activity of neurons. The tDCS induces long-lasting changes in cortical excitability in a reversible manner. M1 excitability is enhanced by anodal and decreased by cathodal stimulation. ${ }^{9-11}$ The after effects of DC stimulation on motor evoked potentials (MEPs) are abolished for both anodal and cathodal polarities using NMDA receptor antagonists, such as dextromethorphan. Mechanisms of action may involve changes in the synaptic efficacy of excitatory projections synapsing onto corticospinal neurons (for a recent review ${ }^{12}$ ). However, there is no effect of dextromethorphan during tDCS, suggesting that the polarization of neurons is the key feature during this period. The $\mathrm{Na}^{+}$ channel antagonist carbamazepine blocks the effect of anodal tDCS on MEPs both during and after stimulation, and this is in line with the idea that anodal tDCS depolarizes neurons by increasing $\mathrm{Na}^{+}$ion influx. Nevertheless, carbamazepine had no effect on MEP amplitudes after cathodal tDCS, indicating that $\mathrm{K}^{+}$channel-dependent hyperpolarization of the membrane may play a vital role.

Transcranial random noise stimulation (tRNS) of the human brain is a novel technique. ${ }^{13}$ During tRNS, a random electrical oscillating spectrum is applied over the M1. The tRNS induces excitability increases lasting up to 60 minutes poststimulation. The tRNS-induced plasticity is understood to be modulated by the continual activation and rectification of voltage-gated sodium channels. ${ }^{14}$ Other proposed mechanisms of action include a modifying of the signal-to-noise ratio in the central nervous system.

A recent study ${ }^{15}$ found that the responses of $M e t$ allele carriers were generally showing a decreased response to three different stimulation protocols: TBS, homeostatic plasticity in the tDCS/1 Hz rTMS model, ${ }^{16}$ paired associative stimulation (PAS), ${ }^{17}$ compared with the response of Val66Val individuals.

In the current study, we have followed up these observations in a larger subject population using a retrospective genotyping cross correlated with findings of previous studies conducted with our laboratory, exploring the impact of $B D N F$ polymorphisms on M1 plasticity induced by anodal/cathodal tDCS and tRNS, which are modulatory stimulation approaches on the one hand, and iTBS, an activity-inducing protocol, on the other. The data suggest that depending on the specific plasticity induction protocol, $B D N F$ polymorphisms produce clearly discernable quantitatively and qualitatively different results. This suggests that there exists a putative link between the expression of particular polymorphisms in the human central nervous tissue, and the observable after effects of transcranial electrical stimulation methods.

\section{Materials and methods}

\section{Subjects}

Altogether, the data of 64 healthy volunteers participating in 87 experimental sessions (36 men; age range: 19-42 years) were included in this retrospective analysis study after giving written informed consent. Demographical data 
Table 1 Demographics of volunteers included in experiments

\begin{tabular}{|c|c|c|c|c|c|c|c|c|}
\hline \multirow{2}{*}{$\frac{\text { Demographic }}{\text { Genotype }}$} & \multicolumn{2}{|l|}{ iTBS } & \multicolumn{2}{|c|}{ Anodal tDCS } & \multicolumn{2}{|c|}{ Cathodal tDCS } & \multicolumn{2}{|l|}{ tRNS } \\
\hline & Val/Val & Val/Met & Val/Val & Val/Met & Val/Val & Val/Met & Val/Val & Val/Met \\
\hline No. of subjects & 10 & 5 & 14 & 10 & 11 & 8 & 21 & 8 \\
\hline Female/male & $7 / 3$ & $3 / 2$ & $7 / 7$ & $4 / 6$ & $5 / 6$ & $3 / 5$ & $11 / 10$ & $5 / 3$ \\
\hline
\end{tabular}

are presented in Table 1. None of the subjects had any neurologic/psychologic disorders, none had metallic implants or implanted electric devices, or took any medications regularly. All subjects were right handed. ${ }^{18} \mathrm{We}$ conform to the Declaration of Helsinki (last modified in 2004), and the experimental protocol was approved by the Ethics Committee of the University of Göttingen.

Unfortunately, because of the retrospective nature of the study, not all the participants gave blood, especially those who had already participated in previously published studies conducted by our laboratories. Some of the subjects were already unavailable or refused to participate in the genotyping study. Furthermore, not all the subjects participated in all the stimulation conditions. Therefore, the number of subjects in the different experimental sessions is diverse.

\section{$B D N F$ genotyping}

Whole blood was taken into EDTA tubes and DNA was extracted using standard procedures. Primer sequences and polymerase chain reaction (PCR) conditions are available on request. The PCR was checked for success on an agarose $/ 2 \times$ TBE gels. Restriction fragment length polymorphism (RFLP) analysis was performed by digesting the PCR product with the restriction enzyme Hsp92II. RFLP conditions are available on request. Restriction products were electrophoresed on a $2 \%$ agarose gel and visualized using a transilluminator and ethidium bromide staining. After digestion, the $\mathrm{Val}$ allele (G) gave two fragments, 57 and $217 \mathrm{bp}$, whereas the Met allele (A) gave three, 57, 77, and $140 \mathrm{bp}$. The 64 participants were genotyped as follows: 46 participants were found to be homozygous for the $\mathrm{Val}$ allele (Val66Val), 15 were Val66Met heterozygotes, and 3 were homozygous for the Met allele. The Met homozygotes were not included in the study, as only two participated in one of the studies.

\section{Electrical and magnetic stimulation: tRNS, tDCS, and iTBS}

Electrical stimulation was delivered by a battery-driven electrical stimulator (DC-Stimulator-Plus, NeuroConn $\mathrm{GmbH}$, Ilmenau, Germany) through conductive-rubber electrodes, placed in two saline-soaked sponges. The stimulation electrode was placed over the left M1, which was determined by single-pulse TMS. The reference electrode was placed over the contralateral orbit. The stimulation intenisty was $1 \mathrm{~mA}$.

\section{tRNS}

Twenty-nine subjects participated in this study ( 8 heterozygotes). In the stimulation mode "noise," there is a random level of current generated for every sample. ${ }^{13}$ The random numbers are normally distributed; the probability density function follows a bell-shaped curve. In the frequency spectrum, all coefficients have a similar size. The noise signal contains all frequencies up to half of the sampling rate, namely, a maximum of $640 \mathrm{~Hz}$. The size of the stimulation electrode was $4 \times 4 \mathrm{~cm}$ and the reference electrode was $6 \times$ $14 \mathrm{~cm}$. The tRNS was applied for 10 minutes.

\section{tDCS}

Twenty-four subjects participated in this study (24 anodal stimulations were performed, including 10 heterozygotes and 19 cathodal stimulations, including 8 heterozygotes). Here, the electrode size was $5 \times 7 \mathrm{~cm}$. Stimulation was performed for 7-9 and 13 minutes (for anodal and cathodal stimulation, respectively). ${ }^{9-11}$

\section{iTBS}

Fifteen subjects participated in this study (5 heterozygotes). iTBS was applied using a Magstim Super Rapid (Magstim Company, Whiteland, Wales, UK) with a standard, figureof-eight coil and MagPro stimulator (Medtronic, Denmark) with an outer half-coil radius of $75 \mathrm{~mm}$, with a posterioranterior-posterior current flow in the coil. Stimulus intensity was $80 \%$ of active motor threshold (AMT). AMT was defined as the lowest stimulus intensity at which three of six consecutive stimuli elicited reliable MEP $(\sim 200 \mu \mathrm{V}$ in amplitude) in the tonically contracting the right first dorsal interosseus (FDI) or the abductor digiti minimi (ADM) muscle. ${ }^{19}$ The pattern of rTMS consisted of bursts containing three pulses at $50 \mathrm{~Hz}$ repeated at 200-millisecond intervals (i.e., at $5 \mathrm{~Hz}$ ). A 2-second train of TBS was repeated every 10 seconds for a total of 190 seconds (600 pulses). ${ }^{7}$

\section{Experimental design}

Subjects were seated in a comfortable reclining chair with a mounted headrest throughout the experiments. Within each type of experimental session, the measurements were always performed by the same experienced investigator. The experiments were performed during daytime (8:0018:00) hours. The blood sampling was performed on nonexperimental days, and the time between the last experimental session and the taking of blood samples was between 1 day and 2 months. To detect current-driven 
changes in excitability, MEPs of the right ADM or FDI were recorded after stimulation of its motor-cortical representation using single-pulse TMS. These were induced using a Magstim 200 or using MagPro stimulator magnetic stimulator (previously described), with a figure-of-eight standard magnetic coil (diameter of one winding, $70 \mathrm{~mm}$ ). Surface electromyography (EMG) was recorded from the right $\mathrm{FDI}$ and $\mathrm{ADM}$ muscle through a pair of $\mathrm{Ag}-\mathrm{AgCl}$ electrodes in a belly-tendon montage. Raw signals were amplified, band-pass filtered ( $2 \mathrm{~Hz}-3 \mathrm{kHz}$; sampling rate 5 $\mathrm{kHz}$ ), digitized with a micro 1401 AD converter (Cambridge Electronic Design, Cambridge, UK) controlled by Signal Software (version 2.13). Complete relaxation was controlled through visual feedback of EMG activity. The coil was held tangentially to the skull, with the handle pointing backward and laterally at $45^{\circ}$ from the midline, resulting in a posterior-anterior current flow direction in the brain. The optimum position was defined as the site where TMS resulted consistently in the largest MEP in the resting muscle.

The intensity required to evoke a MEP of approximately $1 \mathrm{mV}$ peak-to-peak amplitude (SI $1 \mathrm{mV}$ ) and a baseline of TMS-evoked MEPs (40 stimuli) were recorded at $0.25 \mathrm{~Hz}$ before stimulation. After plasticity-inducing stimulation, 40 single test-pulse MEPs were recorded at $0.25 \mathrm{~Hz}$, that is, approximately 0 minute and then every 5 minutes up to 30 minutes poststimulation and finally at 60 minutes.

\section{Data analyses}

Repeated measures analysis of variances (ANOVAs) with the between subject factor GENOTYPE, the within subject factor TIME $(0,5,10,15,20,25,30$, and 60 minutes poststimulation), and the dependent variable MEP amplitude were used to compare the effect of induced neuroplasticity and different stimulation methods among individuals. Baseline-normalized data were used. Effects were considered significant if $P<.05$. Reported $P$ values associated with the $F$ statistics are adjusted via the Greenhouse-Geisser correction ( $d f$ values are rounded). Post hoc Student $t$-test (independent) was performed whenever the genotype or the interaction was significant. In addition, a Student $t$-test was used to compare baseline with poststimulation MEP amplitudes within one condition and considering only one genotype. The results were not Bonferroni corrected for multiple testing. All data are given as means \pm SEM.

\section{Results}

\section{iTBS}

In this experiment we tested whether the Val/Met polymorphism in the $B D N F$ gene affected the response to iTBS. The normalized MEP data from 15 individuals are plotted in Figure 1. The ANOVA of the normalized data revealed a significant main effect of GENOTYPE $\left(F_{1,13}=10.08\right.$; $P<.001)$ and TIME $\left(F_{4,52}=2.88 ; P=.03\right.$; Epsilon $=$ 0.57). The GENOTYPE $\times$ TIME interaction was not significant $\left(F_{4,52}=0.95 ; P=.44\right.$; Epsilon $\left.=0.57\right)$. There was a significant increase in MEPs after iTBS in the Val66 Val individuals during the first 25 minutes $(2.67 \times 4.75$, $P<.03$ at all timepoints), but not in the Val66Met group.

\section{tDCS}

Here, we tested whether the Val/Met polymorphism affected the response to anodal/cathodal tDCS. With regard to anodal stimulation two-way ANOVA of the normalized data revealed no significant main effect of GENOTYPE $\left(F_{1,22}=2.47 ; P=.13\right)$ and $\operatorname{TIME}\left(F_{5,112}=2.12 ; P=.08\right.$; Epsilon $=0.56)$; however, the GENOTYPE $\times$ TIME interaction was significant $\left(F_{5,112}=2.38 ; P=.05\right.$; Epsilon $=$ $0.56)$ (Figure 2$)$. The excitability difference was significant at $25(P=.04)$ and $60(P=.02)$ minutes poststimulation.
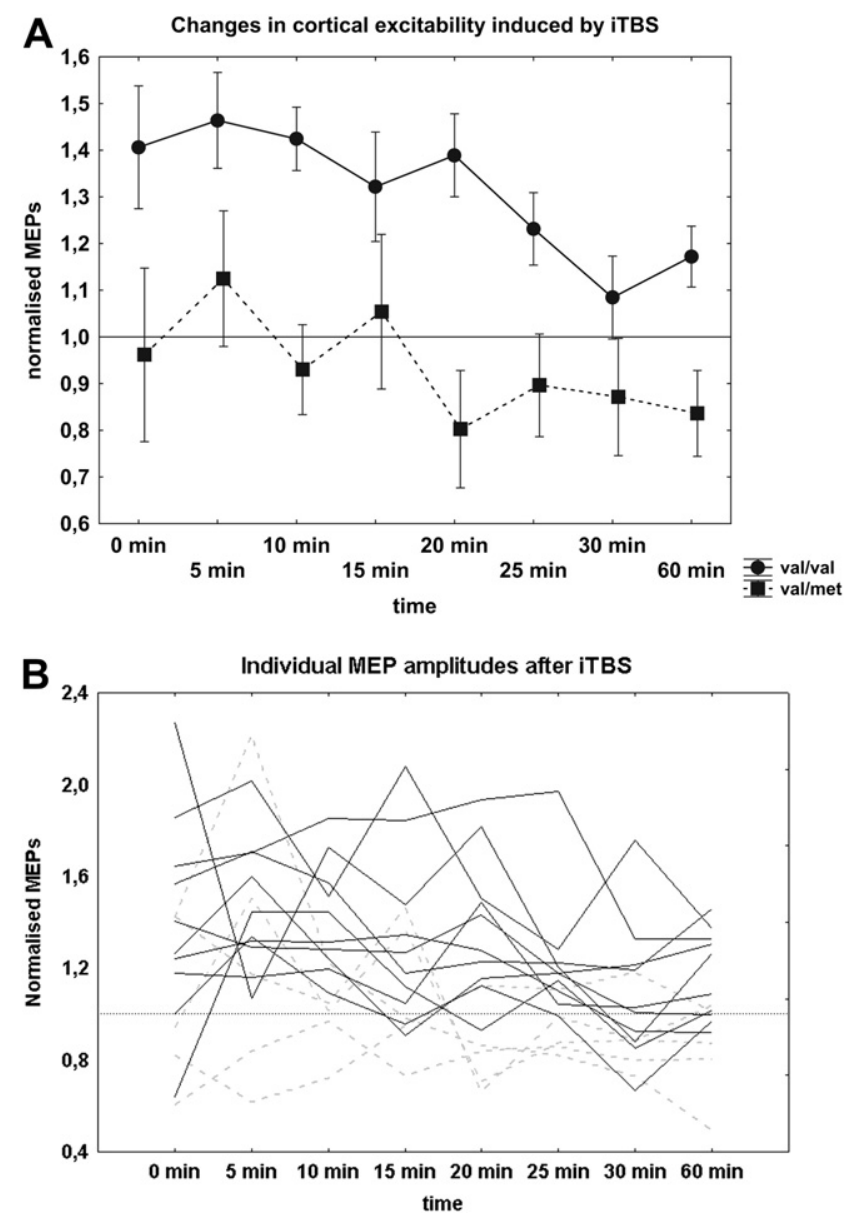

Figure $1 \mathbf{A}$, Effect of $B D N F$ Val66Met polymorphism on cortical excitability in response to iTBS. There was a significant increase in MEPs after iTBS in the Val66Val individuals, but not in the Val66Met group. Data are mean ( \pm SEM) peak-topeak amplitudes of MEP. B, Individual MEPs after iTBS. 
There was a significant increase in MEPs after anodal tDCS in both groups but the excitability enhancement was more pronounced in the Val66Met group ( Val/Val group: during the first 20 minutes $2.39 \times 4.65, P<.03$; Val/Met group: during the first 60 minutes $2.67 \times 4.79, P<.01$ at all timepoints).

With regard to cathodal stimulation, two-way ANOVA of the normalized data revealed no significant main effect of GENOTYPE $\left(F_{1,17}=1.3 ; P=.2\right)$; however, the TIME was significant $\left(F_{4,70}=5.5 ; P=.005\right.$; Epsilon $\left.=0.59\right)$. The GENOTYPE $\times$ TIME interaction was not significant $\left(F_{4,70}=1.5 ; P=.22\right.$ Epsilon $\left.=0.59\right)$. There was a significant decrease in MEPs after cathodal tDCS in both groups. However, when the poststimulation MEP amplitudes were compared with baseline MEPs, the induced cortical inhibition was more pronounced in the Val66Met group than in the Val66Val group (Val/Val group: during the first 20
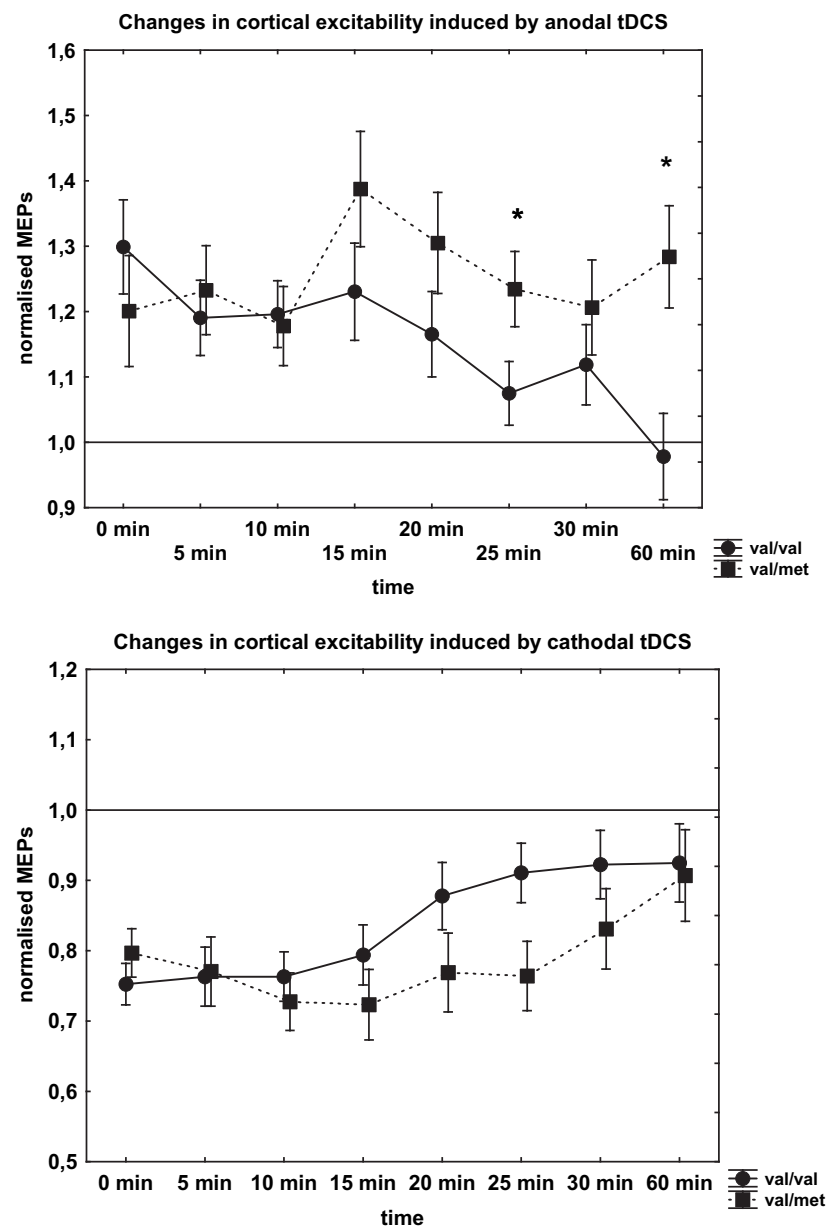

Figure 2 Effect of $B D N F$ Val66Met polymorphism on cortical excitability in response to cathodal and anodal tDCS. There was a significant increase in MEPs after anodal tDCS in both groups and excitability enhancement was more pronounced in the Val66Met group. With regard to the cathodal effect, there was a significant decrease in MEPs after cathodal tDCS in both groups showing a tendency to being stronger in the Val66Met group. Data are mean ( \pm SEM) peak-to-peak amplitudes of MEP. minutes $2.38 \times 7.72, P<.03$; Val/Met group: during the first 30 minutes $3.38 \times 9.91, P<.003$ ).

\section{tRNS}

In this study, we tested whether the Val/Met polymorphism in the $B D N F$ gene affected responses to tRNS. The data from 29 individuals are plotted in Figure 3. ANOVA of the normalized data revealed no significant main effect of GENOTYPE $\left(F_{1,27}=0.02 ; P=.88\right)$, and TIME $\left(F_{3,83}=\right.$ $0.8 ; P=.48$; Epsilon $=0.441)$. The GENOTYPE $\times$ TIME interaction was not significant $\left(F_{3,83}=0.6 ; P=\right.$ .59 ; Epsilon $=0.441)$. There was a significant increase in MEPs after tRNS in both groups (Val/Val group: during all timepoints $2.89 \times 5.43, P<.003$; Val/Met group: except the 25 minutes at all timepoints $2.50 \times 3.42, P<.02$ ).

\section{Discussion}

Each laboratory studying neuroplasticity induced by transcranial stimulation methods is aware of "responders" and "nonresponders," frequently attributed to their ability to completely relax. Indeed, the state of preinnervation plays a central role in inducing plastic after effects. ${ }^{20}$ Recently new light has been shed on a healthy individual's response to external stimulation by drawing attention to the individual's genetic state. The current data from our retrospective genotyping study confirm and extend existing results ${ }^{15}$ demonstrating that the response of healthy subjects to cortical plasticity-inducing protocols used in M1 is associated with polymorphisms in the $B D N F$ genotype and that it might be appropriate to choose the type of stimulation (e.g., stimulation - TBS/PAS versus modulation - tDCS/tRNS) according to the individual's genetic state.

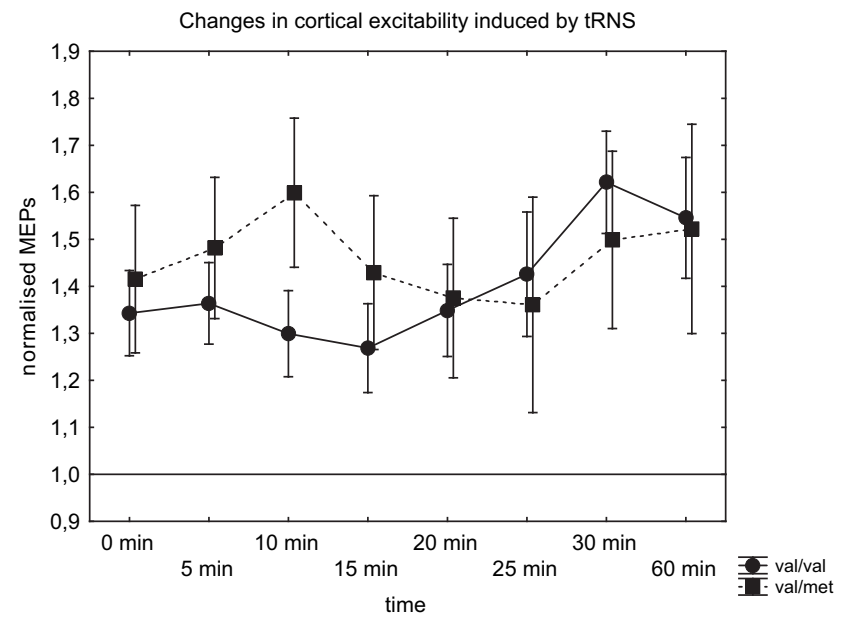

Figure 3 Effect of $B D N F$ Val66Met polymorphism on cortical excitability in response to RNS. There was a significant increase in MEPs after tRNS in both groups. Data are mean ( \pm SEM) peak-to-peak amplitudes of MEP. 
With regard to TBS, continuous TBS (cTBS) induces inhibitory and iTBS induces facilitatory plasticity in nongenotyped healthy controls. ${ }^{7}$ In genotyped Val66Val individuals, ${ }^{15}$ iTBS facilitates MEPs for 30 minutes; however, the after effects of iTBS are reduced or absent in subjects carrying the Met allele of the BDNF gene. Our results confirm these data. This genetically driven difference in the efficacy of rTMS to induce plasticity seems to be relevant for therapeutic application. A recent study investigated the role of the $B D N F$ polymorphism in response to rTMS in a group of 36 patients affected by mood disorders. ${ }^{21}$ rTMS treatment significantly improved depression symptomatology and the response was significantly greater in Val66Val homozygotes when compared with Met allele carriers. It is likely that Met carriers are less susceptible to the effects of iTBS/rTMS than the Val66Val individuals. This might arise because Val66Met individuals lack any response to rTMS/iTBS and/or they have a higher threshold with regard to the stimulation intensity/duration relation and the induced after effects.

The foremost new finding presented in this study addresses the opposing effects of tDCS in the Val66Met individuals when compared with iTBS. Indeed, we observed that the effects of DC stimulation were more pronounced in Met66Val individuals than in Val66Val subjects. Only one previous study has examined the relationship between $B D N F$ genotyping and the characteristic after effects of tDCS. ${ }^{15}$ In this study, a metaplastic conditioning protocol was used, in which 10- minute cathodal tDCS was followed by 900 subthreshold TMS pulses at 1 $\mathrm{Hz}{ }^{16}$ In this study, cathodal tDCS produced the same amount of LTD-like suppression of corticospinal activity in all subjects. In addition, we did not observe any difference between homo- and heterozygotes immediately poststimulation, but did 20-50 minutes later. Similar to anodal tDCS, we have observed facilitation post-tRNS (which is the normal phenomenon described in a nongenotyped population $^{13}$ ) in both groups. However, this tRNS after effect appears less robust concerning genetic $B N D F$ variants (compared with the anodal effect), which may be accounted for by differing mechanisms of activation. The tDCS modifies the transmembrane neuronal potential directly, thus modulating the firing rate of individual neurons. ${ }^{22}$ In contrast, tRNS may possibly interfere with ongoing oscillations and neuronal activity in the brain through the activation of sodium channels by high-frequency stimulation. ${ }^{14}$ These two methods have more a "modulatory" effect as opposed to the "stimulatory" effects of PAS and rTMS/ TBS. PAS comprises slow-rate repetitive low-frequency median nerve stimulation combined with TMS over the contralateral M1. Concerning PAS, in Val66met subjects, the stimulation produced no significant increase in MEP amplitude. ${ }^{15}$ Similarly, with regard to iTBS/cTBS, there were no MEP increase (iTBS) and decrease (cTBS) poststimulation in the Val66Met individuals. This demonstrates that "modulatory" methods and "stimulatory" (or subthreshold/suprathreshold stimulation) methods act on different neural circuits; they possess different preand postsynaptic effects, which are differentially responsive because of the $B D N F$ polymorphism, and may have a significant effect on $B D N F$ release. $B D N F$ has been shown to elicit pronounced effects on quantal neurotransmitter release at the synapse through presynaptic mechanisms. ${ }^{23}$ $B D N F$ modifies the strength of neuronal connections by "fine-tuning" synaptic activity and improving signalto-noise ratio through direct actions at presynaptic terminals. With regard to the possible mechanism of the pronounced tDCS after effects in Val66Met individuals, this potentially means less "noise reduction" in the Met allele carriers.

In addition to these mechanisms, we could also consider that the different types of stimulation act differentially on pro- $B D N F$ release depending on their primary molecular "hot spot," and therefore the extracellular equilibrium of pro- $B D N F-\mathrm{m} B D N F$ might be also altered. ${ }^{24}$

Previous studies have suggested that $B D N F$ polymorphisms have both anatomic and functional/behavioral consequences in healthy human populations. Egan et al. ${ }^{4}$ reported that when compared with Val66Val subjects, Met carriers have smaller hippocampal volumes and reduced grey matter in the frontal cortex. Furthermore, in behavioral studies, Met66Met homozygotes have impaired episodic memory, whereas Val66Met heterozygotes had impaired recognition accuracy of visual scenes in a declarative memory task. Hariri et al. $^{25}$ reported that Met carriers exhibit relatively diminished hippocampal engagement and impairment of performance in comparison to $\mathrm{Val}$ homozygotes during both encoding and retrieval processes in a declarative memory task. In a functional/behavioral study single-pulse TMS has been used to demonstrate that the $B D N F$ genotype is associated with dynamic changes in excitability of the M1, that occur after practicing a motor task. ${ }^{26}$ The authors found that in healthy subjects with a Val66Met polymorphism, training-dependent increase in the amplitude of MEPs was reduced. However, it was also found that the $B D N F$ Val66Met polymorphism is not associated with variation in working memory in healthy adolescents. ${ }^{27}$ This study examined a large sample $(n=785)$ of adolescent twin-pairs and siblings. A range of measures (event-related potential, general performance, and reaction time) obtained from a delayed-response workingmemory task showed no correlation between genotype and working memory performance in the given task.

Although many studies report well-characterized interactions of $B D N F$ and transcranial electrical stimulation on memory function and retrieval, there are tentative studies demonstrating the role of $B D N F$ and motor behavior. In a recent study by Ploughman et al., ${ }^{26}$ rats showed significant recovery of skilled reaching, observed in a staircase task, after sustaining focal ischemia. Levels of $B D N F$ known to increase are exercise and exposure to an enriched environment in both animal and human models. ${ }^{28,29}$ Studies 
in animal models suggest that there exists a link between the ability to recover motor function during rehabilitation after a physical trauma that can be potentially transferable to human models of rehabilitation postinjury. Another recent study reports that $B D N F$ promotes synaptic stability through the modulation neuronal firing patterns, which suggests that $B D N F$ may play an integral role in the rearrangement of synaptic circuitry post injury and during rehabilitation. ${ }^{30}$

Many previous studies have pointed out the variability of individual responses to rTMS/tDCS protocols. A number of factors have already been described that may contribute to intraindividual variation, such as the subject's age, ${ }^{31}$ gender, ${ }^{32}$ circadian rhythmicity, ${ }^{33}$ the menstrual cycle, ${ }^{34}$ or the prior history of brain activation. ${ }^{35,36}$ Indeed, the state of preinnervation plays a central role in inducing plastic after effects. ${ }^{20}$ According to other reports including our current work, it is a well-established notion that polymorphisms in the $B D N F$ gene can directly influence synaptic plasticity in the adult human brain. Therefore, it would be useful to include this variable as a potential cofactor in the analysis of the behavioral/physiologic data. In addition, it raises the question as to whether this polymorphism is responsible for the inefficacy of rTMS/tDCS treatment in some neurologic populations. Certainly, in addition to the Val66Met single nucleotide polymorphism, we have to consider that other variants of the polymorphism (not just the alleles) may also be responsible for the differences that we can see in the responses to stimulation after effects. $^{37}$ Not only $B D N F$ variants, but the interplay between other genes within the same loci may have a pronounced effect on the stability and size of MEP amplitudes, which in turn, may exert effects on the anticipated after effects poststimulation. At present, approximately 100 candidate genes influencing human brain functions and cognition have been reported. ${ }^{38}$ In addition, the involvement of neurotransmitter receptors ${ }^{16}$ might also be associated with a given allele, and therefore varying protocols might be differentially affected by polymorphismdependent neuronal plasticity. Furthermore, it is not yet known whether $B D N F$ receptors (TrkB and p75) are ubiquitously expressed throughout the cortex, as they are in body tissues.

The limitation of our study is that it is a retrospective analysis, and therefore the number, age, and gender distribution of the homo- and heterozygote groups were not matched. However, the subjects were within the same age-range and the ratio of females/males was not significantly different between the two populations. Therefore, the current results should be considered as preliminary and need to be replicated and validated in a dedicated prospective study. Although our findings support the role of $B D N F$ as a major modulator of cortical plasticity in humans, further work needs to be performed to fully understand the direct influence of $B D N F$ and its variants on mechanisms of neuronal plasticity. Currently, there exists only a single study demonstrating the effects TMS on the expression of c-Fos and $B D N F$, and although this study also supports the causative link between $B D N F$ and electrical stimulation, ${ }^{39}$ more specific work on animal models needs to be performed to further define this relationship.

\section{Acknowledgment}

We thank John Rothwell for his constructive comments and advice.

\section{References}

1. Figurov A, Pozzo-Miller LD, Olafsson P, Wang T, Lu B. Regulation of synaptic responses to high-frequency stimulation and LTP by neurotrophins in the hippocampus. Nature 1996;381:706-709.

2. Woo NH, Teng HK, Siao CJ, et al. Activation of p75NTR by proBDNF facilitates hippocampal long-term depression. Nat Neurosci 2005;8:1069-1077.

3. Bramham CR, Messaoudi E. BDNF function in adult synaptic plasticity: the synaptic consolidation hypothesis. Prog Neurobiol 2005; 76:99-125.

4. Egan MF, Kojima M, Callicott JH, et al. The BDNF val66met polymorphism affects activity-dependent secretion of BDNF and human memory and hippocampal function. Cell 2003;112:257-269.

5. Pezawas L, Verchinski BA, Mattay VS, et al. The brain-derived neurotrophic factor val66met polymorphism and variation in human cortical morphology. J Neurosci 2004;24:10099-10102.

6. Ziemann U, Paulus W, Nitsche MA, et al. Consensus: motor cortex plasticity. Brain Stimulation 2008;1:164-182.

7. Huang YZ, Edwards MJ, Rounis E, Bhatia KP, Rothwell JC. Theta burst stimulation of the human motor cortex. Neuron 2005;45: 201-206.

8. Huang YZ, Chen RS, Rothwell JC, Wen HY. The after-effect of human theta burst stimulation is NMDA receptor dependent. Clin Neurophysiol 2007;118:1028-1032.

9. Nitsche MA, Paulus W. Excitability changes induced in the human motor cortex by weak transcranial direct current stimulation. J Physiol 2000;527:633-639.

10. Nitsche MA, Paulus W. Sustained excitability elevations induced by transcranial DC motor cortex stimulation in humans. Neurology 2001;57:1899-1901.

11. Nitsche MA, Fricke K, Henschke U, et al. Pharmacological modulation of cortical excitability shifts induced by transcranial direct current stimulation in humans. J Physiol 2003;553:293-301.

12. Nitsche MA, Cohen LG, Wassermann EM, et al. Transcranial direct current stimulation: state of the art 2008. Brain Stimulation 2008;1: 206-223.

13. Terney D, Chaieb L, Moliadze V, Antal A, Paulus W. Increasing human brain excitability by transcranial high-frequency random noise stimulation. J Neurosci 2008;28:14147-14155.

14. Bromm B. Die Natrium-Gleichrichtung der unterschwellig erregten Membran in der quantitative Formulierung der Ionentheorie. Pflügers Arch 1968;302:233-244.

15. Cheeran B, Talelli P, Mori F, et al. A common polymorphism in the brain-derived neurotrophic factor gene (BDNF) modulates human cortical plasticity and the response to rTMS. J Physiol 2008;586: 5717-5725.

16. Siebner HR, Lang N, Rizzo V, et al. Preconditioning of low-frequency repetitive transcranial magnetic stimulation with transcranial direct current stimulation: evidence for homeostatic plasticity in the human motor cortex. J Neurosci 2004;24:3379-3385.

17. Classen J, Wolters A, Stefan K, et al. Paired associative stimulation. Suppl Clin Neurophysiol 2004;57:563-569. 
18. Oldfield RC. The assessment and analysis of handedness: the Edinburgh inventory. Neuropsychologia 1971;9:97-113.

19. Rothwell JC, Hallett M, Berardelli A, et al. Magnetic stimulation: motor evoked potentials: the International Federation of Clinical Neurophysiology. Electroencephalogr Clin Neurophysiol Suppl 1999;52:97-103.

20. Antal A, Terney D, Poreisz C, Paulus W. Towards unravelling task-related modulations of neuroplastic changes induced in the human motor cortex. Eur J Neurosci 2007;26:2687-2691.

21. Bocchio-Chiavetto L, Miniussi C, Zanardini R, et al. 5-HTTLPR and BDNF Val66Met polymorphisms and response to rTMS treatment in drug resistant depression. Neurosci Lett 2008;437:130-134.

22. Bindman LJ, Lippold OCJ, Redfearn JWT. The action of brief polarizing currents on the cerebral cortex of the rat (1) during current flow and (2) in the production of long-lasting after-effects. J Physiol 1964; 172:369-382.

23. Tyler WJ, Perrett SP, Pozzo-Miller LD. The role of neurotrophins in neurotransmitter release. Neuroscientist 2002;8:524-531.

24. Brunoni AR, Boggio PS, Fregni F. Can the 'yin and yang' BDNF hypothesis be used to predict the effects of rTMS treatment in neuropsychiatry? Med Hypotheses 2008;71:279-282.

25. Hariri AR, Goldberg TE, Mattay VS, et al. Brain-derived neurotrophic factor val66met polymorphism affects human memory-related hippocampal activity and predicts memory performance. J Neurosci 2003; 23:6690-6694.

26. Kleim JA, Chan S, Pringle E, et al. BDNF val66met polymorphism is associated with modified experience-dependent plasticity in human motor cortex. Nat Neurosci 2006;9:735-737.

27. Hansell NK, James MR, Duffy DL, et al. Effect of the BDNF V166M polymorphism on working memory in healthy adolescents. Genes Brain Behav 2007;6:260-268.

28. Ploughman M, Windle V, MacLellan CL, et al. Brain-derived neurotrophic factor contributes to recovery of skilled reaching after focal ischemia in rats. Stroke 2009;40:1490-1495.
29. Zoladz JA, Pilc A, Majerczak J, et al. Endurance training increases plasma brain-derived neurotrophic factor concentration in young healthy men. J Physiol Pharmacol Suppl 2008;59(7):119-132.

30. Davis-López de Carrizosa MA, Morado-Díaz CJ, Tena JJ, et al. Complementary actions of BDNF and neurotrophin-3 on the firing patterns and synaptic composition of motoneurons. J Neurosci 2009; 29:575-587.

31. Müller-Dahlhaus J, Orekhov Y, Liu Y, Ziemann U. Interindividual variability and age-dependency of motor cortical plasticity induced by paired associative stimulation. Exp Brain Res 2008;187: 467-475.

32. Kuo MF, Paulus W, Nitsche MA. Sex differences in cortical neuroplasticity in humans. Neuroreport 2006;17:1703-1707.

33. Sale MV, Ridding MC, Nordstrom MA. Cortisol inhibits neuroplasticity induction in human motor cortex. J Neurosci 2008;28: 8285-8293.

34. Inghilleri M, Conte A, Curr'a A, et al. Ovarian hormones and cortical excitability: an rTMS study in humans. Clin Neurophysiol 2004;115: 1063-1068.

35. Lang N, Siebner HR, Ernst D, et al. Preconditioning with transcranial direct current stimulation sensitizes the motor cortex to rapid-rate transcranial magnetic stimulation and controls the direction of after-effects. Biol Psychiatry 2004;56:634-639.

36. Silvanto J, Muggleton N, Walsh V. State-dependency in brain stimulation studies of perception and cognition. Trends Cogn Sci 2008;12: 447-454.

37. Klein TA, Neumann J, Reuter M, et al. Genetically determined differences in learning from errors. Science 2007;318:1642-1645.

38. de Geus E, Goldberg T, Boomsma DI, Posthuma D. Imaging the genetics of brain structure and function. Biol Psychol 2008;79:1-8.

39. Zhang X, Mei Y, Liu C, Yu S. Effect of transcranial magnetic stimulation on the expression of c-Fos and brain-derived neurotrophic factor of the cerebral cortex in rats with cerebral infarct. J Huazhong Univ Sci Technolog Med Sci 2007;4:415-418. 


\subsection{Frequency-dependent electrical stimulation of the visual cortex}

Kanai R, Chaieb L, Antal A, Walsh V, Paulus W. Curr Biol. 2008;18(23):1839-43.

In this study tACS was applied over the occipital cortex to ascertain whether the oscillating current could interfere with the ongoing cortical rhythmicity. It was observed that the effects of delivering tACS at $1 \mathrm{~mA}$ in light and dark conditions modulated the perception of phosphenes, and that tACS delivered over the visual cortex in the beta range during the light condition, was most effective in inducing the perception of 'flickering lights'. However, when the frequency range of the stimulation was shifted to the alpha range during the dark condition, the intensity of the 'flickering light' sensation increased. This study indicates that tACS has the potential to entrain ongoing cortical rhythms to an externally applied electrical current. 


\section{Frequency-Dependent Electrical Stimulation of the Visual Cortex}

\author{
Ryota Kanai, ${ }^{1, *}$ Leila Chaieb,, ${ }^{2}$ Andrea Antal, ${ }^{2}$ \\ Vincent Walsh, ${ }^{1}$ and Walter Paulus ${ }^{2}$ \\ ${ }^{1}$ Institute of Cognitive Neuroscience \& Dept of Psychology \\ University College London \\ 17 Queen Square \\ WC1N 3AR, London \\ UK \\ 2Department of Clinical Neurophysiology \\ Georg-August University \\ Robert Koch Straße 40 \\ 37075, Göttingen \\ Germany
}

\section{Summary}

Noninvasive cortical stimulation techniques, such as transcranial magnetic stimulation (TMS) $[1,2]$ and transcranial direct current stimulation (tDCS) [3-6], have proved to be powerful tools for establishing causal relationships between brain regions and their functions [1, 2]. In the present study, we demonstrate that a new technique called transcranial alternating current stimulation (tACS) [7] can interact with ongoing rhythmic activities in the visual cortex in a frequency-specific fashion and induce visual experiences (phosphenes). We delivered an oscillatory current over the occipital cortex with tACS. In order to observe interactions with ongoing cortical rhythms, we compared the effects of delivering tACS under conditions of light ("Light" condition) or darkness ("Dark" condition). Stimulation over the occipital cortex induced perception of continuously flickering light most effectively when the beta frequency range was applied in an illuminated room, whereas the most effective stimulation frequency shifted to the alpha frequency range during testing in darkness. Stimulation with theta or gamma frequencies did not produce any visual phenomena. The shift of the effective stimulation frequency indicates that the frequency dependency is caused by interactions with ongoing oscillatory activity in the stimulated cortex. Our results suggest that tACS can be used as a noninvasive tool for establishing a causal link between rhythmic cortical activities and their functions.

\section{Results and Discussion}

Various frequencies of rhythmic activities are observed in the brain across many species [8]. Oscillatory synchrony plays an essential role in visual attention and perception by dynamically modifying neuronal interactions between brain regions [9-11]. In humans, brain activity measured with electroencephalography (EEG) on the scalp and intracranially recorded local field potentials exhibit typical oscillatory activities at various frequencies. We therefore sought to use frequency-specific brain stimulation to entrain oscillatory activity in the visual cortex. We predicted that weak alternating current applied over

*Correspondence: kanair@gmail.com the scalp would be able to entrain specific EEG frequency bands and induce phenomena specifically related to the functions of a stimulated region and its oscillatory activities. Indeed, a recent study has shown that transcranial application of oscillatory potentials at a low frequency $(0.75 \mathrm{~Hz})$ during slow-wave sleep can enhance the consolidation of declarative memory [12].

It is known from tDCS studies that polarization of cortical neurons with transcranially applied electric currents modulates excitability of visual areas under the stimulation electrode $[13,14]$. Thus, we expected that tACS would also be able to stimulate those areas with the same electrode montages and chose those areas as our target stimulation sites for phosphene induction with tACS. The question of particular interest here is whether phosphene induction would show any physiologically meaningful dependency on stimulation frequency. Thus we used a broad range of parameters, including the theta (4-8 Hz), alpha (8-14 Hz), beta $(14-22 \mathrm{~Hz})$, and gamma $(>30 \mathrm{~Hz})$ ranges. Because EEG displays distinct patterns of dominant frequency as a function of the presence or absence of visual input, we conducted the experiments both in the light and in the dark. Alpha activity recorded over early visual areas is dominant during eyes-closed or in-the-dark resting conditions, whereas in eyes-open, in the light, alpha is suppressed and is replaced by brain activity at higher frequencies, such as the beta range [15-18]. Therefore, differences in the effective tACS stimulation frequencies between the two lighting conditions would be indicative of an interaction between ongoing and induced oscillatory activity. In order to characterize the frequency dependency of tACS over the visual cortex, we measured both subjective and objective measures of cortical excitability by phosphene ratings and threshold measurements, respectively.

To stimulate early visual areas, a small stimulation electrode was placed $4 \mathrm{~cm}$ above the inion and a large reference electrode was placed over the vertex (see Supplemental Experimental Procedures, available online). A larger electrode was used for the reference, in order to minimize the current density under the reference electrode [19].

Eight healthy observers (four male, four female) participated in an experiment in which they were asked to rate the presence or absence and the strength of a tACS-induced phosphene at frequencies ranging from $4 \mathrm{~Hz}$ to $40 \mathrm{~Hz}$. The experiment was conducted as follows: First, we familiarized the observers with the appearance of phosphenes in the light by applying $16 \mathrm{~Hz}$ tACS at a current strength of $1000 \mu \mathrm{A}$ (the maximum current density at the stimulation electrode corresponded to $\left.83 \mu \mathrm{A} / \mathrm{cm}^{2}\right)$. This stimulation was used as the comparator for both phosphene induction in both Light and Dark conditions, with various combinations of stimulation intensity and frequency (see Supplemental Experimental Procedures).

Observers were unaware of the stimulation frequency on any trial, and frequency was randomized within current intensities. For each frequency, observers were tested both in the light and in the dark (each about $5 \mathrm{~s}$ ), consecutively, and were asked to give a rating for the phosphenes in each condition. We alternated the Light and the Dark conditions to avoid buildup of adaptation to darkness across trials [20], given 
A

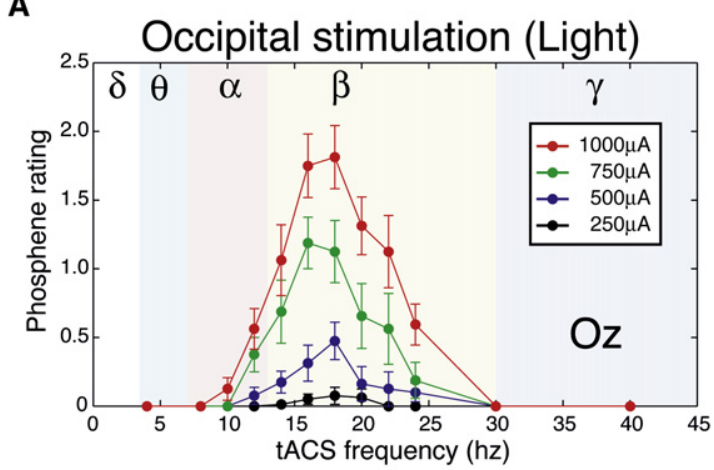

B

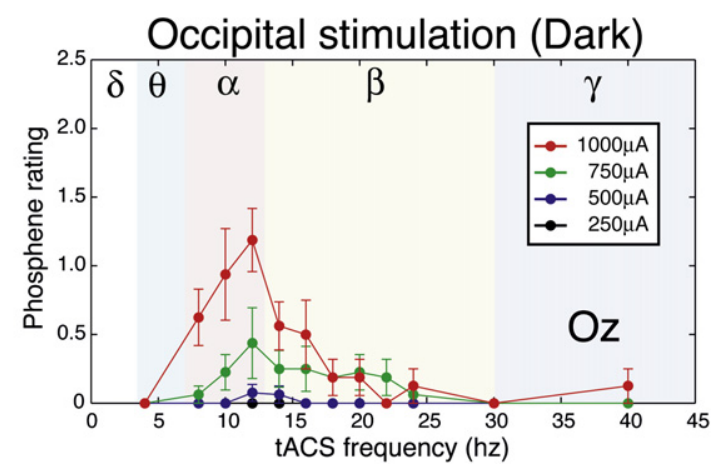

Figure 1. Phosphene Ratings of tACS over Early Visual Cortex

(A) Mean ratings for phosphene strength in the Light condition. Each line corresponds to the rating results for different stimulation intensities (see inset). The error bars correspond to one standard error of the mean (SEM). (B) Mean ratings for phosphene strength in the Dark condition.

that slow adaptation to darkness is known to change sensitivity to TMS-induced phosphenes [21]. After their report of the presence and strength of phosphenes, observers were also asked to describe the phosphene(s) qualitatively.

\section{Phosphene Rating}

In the Light condition, phosphenes were most prominent with tACS in a frequency range of $14 \mathrm{~Hz}$ to $20 \mathrm{~Hz}$ (Figure 1A), which corresponds to the intermediate beta frequency of the EEG. The perception of phosphenes was also restricted to the beta range when tACS was applied at a weaker intensity (e.g., $250 \mu \mathrm{A}$ and $500 \mu \mathrm{A}$ ).

When tACS was applied at a high intensity $(1000 \mu \mathrm{A})$ within the beta range, flickering phosphenes were continuously perceived. When tACS was delivered in darkness, the strongest phosphenes were reported during stimulation at alpha frequencies $(10 \mathrm{~Hz}-12 \mathrm{~Hz})$. The beta frequency stimulation, which was most effective in the Light condition, did not produce phosphenes as strong as those seen in the Light condition. Instead, stimulations at alpha frequencies produced stronger phosphenes in the Dark condition (mean rating of $0.92 \pm$ $0.20 \mathrm{SEM})$ than in the Light condition $(0.23 \pm 0.07 \mathrm{SEM})(\mathrm{T}(7)=$ $3.24, p<0.01)$. This shift of the optimal stimulation frequency suggests that the effectiveness of tACS is related to the existing physiological state rather than to the stimulation properties per se. The alpha range is the most dominant frequency in EEG in the absence of visual input [15-18]; thus, it seems that tACS most effectively induces the perception of phosphenes when it is delivered at the known dominant frequency of the area being stimulated.

A three-way repeated-measures ANOVA showed a main effect of stimulation intensity $(F(2,14)=34.4, p<0.001)$, stimulation frequency $(F(11,77)=15.2, p<0.001)$, and lighting condition $(F(1,7)=21.5, p<0.01)$. There was a significant interaction between stimulation frequency and lighting condition $(F(11,77)=13.2, p<0.001)$, supporting the shift of the peak between the Light and Dark conditions. Other interactions were not significant.

During their observations of phosphenes, observers frequently reported qualitatively different phosphenes in the Dark condition. Whereas the phosphenes in the Light condition were perceived as flickering rapidly, phosphenes in the Dark condition were reported to be more diffuse and slowly oscillating. Also, for frequencies at which phosphenes were reported in both Light and Dark conditions, observers reported different positions and orientations of the phosphenes depending on the lighting condition. These observations suggest that the tACS might interact with the visual cortex in a qualitatively different manner depending on the presence or absence of visual stimulation.

\section{Phosphene Thresholds}

In a second set of experiments, we measured the threshold stimulation intensity required for induction of a phosphene with a probability of $50 \%$. Four naive observers (two females and two males) participated in this study. As in the phosphene-rating experiment, two lighting conditions (Light and Dark) and twelve stimulation frequencies $(4 \mathrm{~Hz}-40 \mathrm{~Hz})$ were examined. A threshold was estimated for each of the 48 test conditions with the use of a modified-binary-search adaptive staircase [22].

The results are shown in Figure 2. Consistent with the phosphene-rating experiment, thresholds were lowest in the beta frequency range during testing in the Light condition. During testing in the Dark condition, the most sensitive frequency shifted from the beta range to the alpha range: the most effective frequency was $20 \mathrm{~Hz}$ in the Light condition, whereas it shifted to $10 \mathrm{~Hz}$ in the Dark condition.

These results are consistent with the results of the phosphene-rating experiment. For stimulation conditions in which observers reported a strong phosphene, the thresholds were low. However, there was a small discrepancy in these two measurements. In the threshold-measurement experiment, the most effective frequency in the Light condition was $20 \mathrm{~Hz}$, whereas it was slightly lower $(16 \mathrm{~Hz}-18 \mathrm{~Hz})$ in the phosphene-rating experiment. The most effective frequency in the threshold-measurement experiment in the Dark condition peaked at $10 \mathrm{~Hz}$, whereas the peak for the phosphene-rating experiment was $12 \mathrm{~Hz}$.

Thus far, we have shown in two experiments that the most effective stimulation frequency changes depending on the lighting condition. In the Light condition, the most effective tACS frequency corresponded to the beta range in the EEG, whereas it shifted to the alpha range in the Dark condition. This shift corresponds to the known shift of the dominant EEG frequency [15-18] and supports the idea that tACS interacts with the ongoing oscillatory activities measured in EEG.

\section{Appearances of Phosphenes}

In order to gain insight into the perception of phosphenes, we conducted a separate experiment in which observers were asked to draw the positions and the shapes of phosphenes. 
A

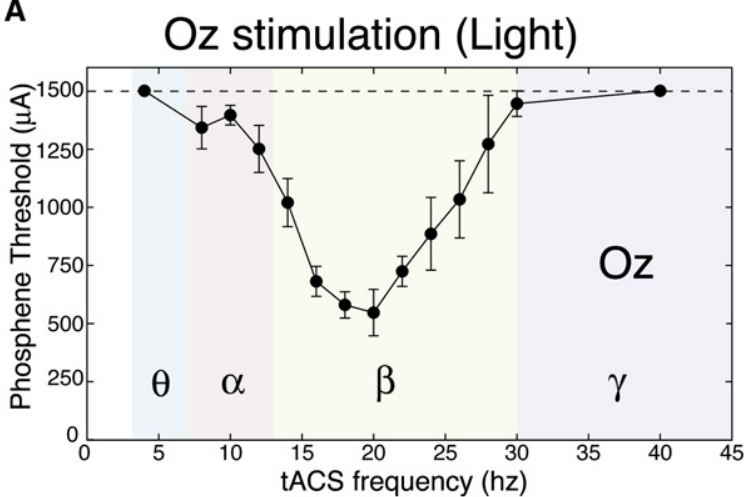

B

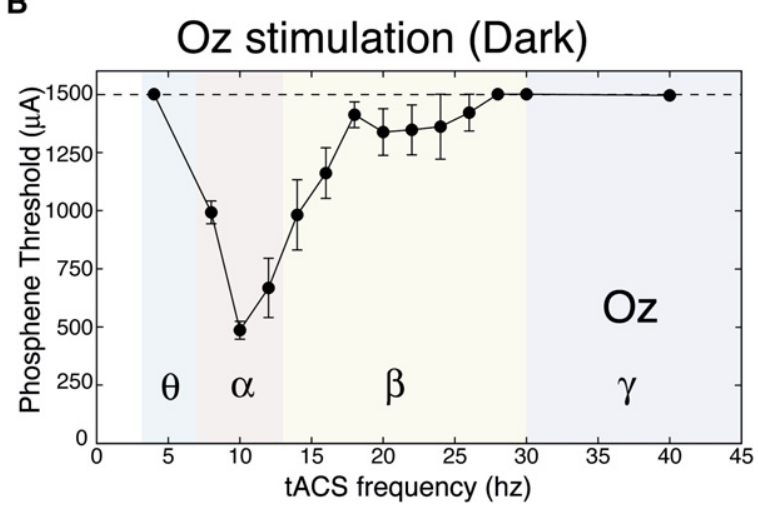

Figure 2. Thresholds for Phosphene Induction

(A) Mean phosphene thresholds in the Light condition. The error bars correspond to one standard error of the mean (SEM).

(B) Mean phosphene thresholds in the Dark condition.

In particular, the position of the phosphene is informative regarding the cortical regions stimulated by alternating current, because the retinotopic organization of early visual areas is well understood $[23,24]$. We obtained drawings of phosphenes induced by occipital stimulation in Light and Dark conditions. To maximize the clarity of phosphenes, we used a stimulation frequency of $18 \mathrm{~Hz}$ for the Light condition and $10 \mathrm{~Hz}$ for the Dark condition.
The drawings of phosphene regions were obtained from six naive observers (Figure 3). All observers reported phosphenes in the periphery of the visual field. The point of phosphenes nearest to fixation was about $15^{\circ}-20^{\circ}$ away. Unlike phosphenes induced by TMS over V1 [25], the phosphenes induced by occipital stimulation were not restricted to the lower visual field; they appeared in both the upper and lower visual fields. In the Dark condition, the phosphene regions generally increased in size.

The fact that phosphenes were reported in the far peripheral vision suggests that tACS stimulated the anterior part of the visual cortex on the medial wall, where the peripheral parts of the visual field are represented. This suggests that current flowed mostly in the cerebrospinal fluid between the two hemispheres and entered the cortex from the anterior part of the visual cortex. Given that the conductivity of cerebrospinal fluid (CSF; $1.25 \sim 1.79 \mathrm{~S} / \mathrm{m}$,) is roughly 3-5 times as high as that of the cortical tissues (white matter $0.25 \mathrm{~S} / \mathrm{m} \sim 0.48 \mathrm{~S} / \mathrm{m}$; gray matter $0.28 \mathrm{~S} / \mathrm{m} \sim 0.7 \mathrm{~S} / \mathrm{m}$ ) [26-28], it is plausible that current flows in the CSF along the medial wall until it is blocked by the posterior part of the corpus callosum (splenium). The idea that current flows primarily along the CSF will be a useful guide in targeting other cortical areas with tACS. However, this needs to be further confirmed with functional brain imaging and neuronal modeling.

In the literature on intracranial electrical stimulation of the visual cortex, most studies have used repetitive pulses with various frequencies and pulse durations [29-32]. In the visual cortex, it has been shown that perceived brightness of phosphenes increases monotonically as the frequency of pulses increases up to $200 \mathrm{~Hz}$ [33]. In the motor cortex, the threshold for evoking a motor response decreased with the frequency of pulsed stimulation up to $90 \mathrm{~Hz}$ and then increased for higher frequencies [34]. Thus, experiments with intracranial pulsed stimulation generally show a monotonic increase in efficacy within the frequency range used in our study (i.e., $<40 \mathrm{~Hz}$ ), whereas our stimulation method showed nonmonotonic frequency dependency.

Although it is difficult to estimate current density at the surface of the visual cortex in our tACS experiments, it is clear that the intensity required for stimulation of the cortical surface via tACS is considerably lower than that required for pulsed stimulation of the cortical surface. For example, in Dobelle and Mladejovsky's study [31], the thresholds for $50 \mathrm{~Hz}$ pulse stimulation on the pial surface were estimated to be on the order of

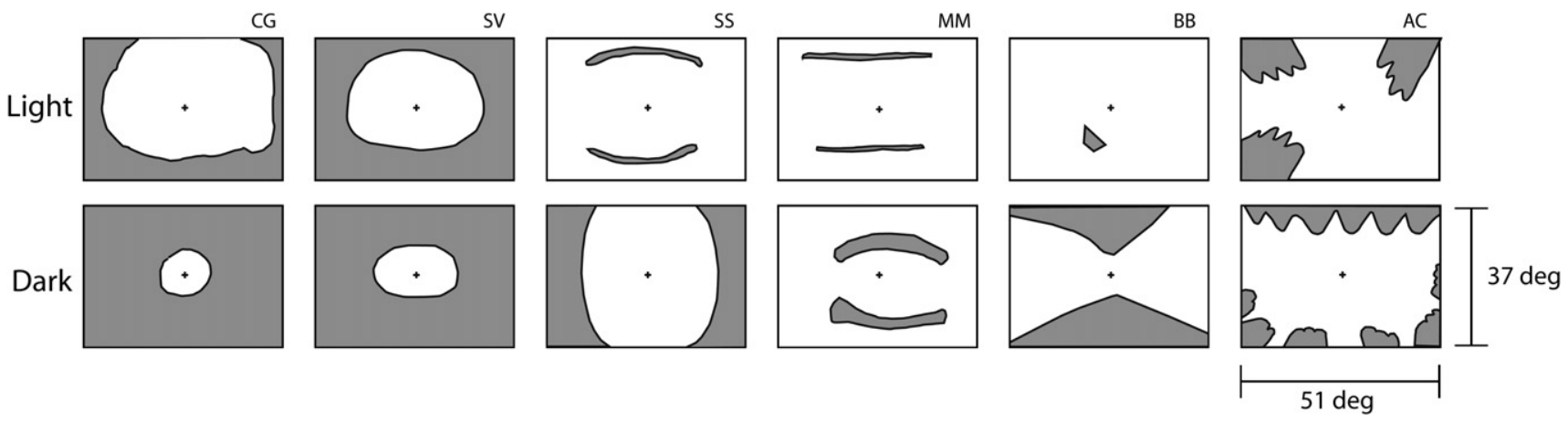

Figure 3. Drawings of Phosphenes

The stimulation frequency was $18 \mathrm{~Hz}$ for the Light condition and $10 \mathrm{~Hz}$ for the Dark condition. The stimulation intensity was $1000 \mu \mathrm{A}$. The phosphenes are represented as the gray regions. Each column corresponds to one observer. The upper panels show the drawings of the phosphenes in the Light condition, and the lower panels show the drawings of the phosphenes in the Dark condition. 
$1000 \sim 5000 \mu \mathrm{A}$ in the visual cortex. Given the volume conduction of the scalp and skull, as well as the distance between stimulation electrode and stimulated cortical surface, in our study, the current that reached the surface of the cortex must be much smaller than that of the studies with pulsed stimulation on the pial surface.

In earlier studies of transcranial electric stimulation (TES), brief, high-voltage electric shocks were used to stimulate the cortex [35-37]. In those studies, much stronger current intensity was required for induction of the perception of phosphenes $(800 \mathrm{~V}$, with a peak current of $50 \mathrm{~A})$, as compared to our present study (5-15 V, with a peak current of 1.0-1.5 A). This suggests that the visual cortex is more sensitive to electrical stimulations at the frequencies observed in EEG; these frequencies are presumably supported by the visual cortex as a system for a given state (e.g., dark versus light).

The first study with tACS [7] attempted to stimulate the motor cortex. However, the results were somewhat disappointing; no effects of tACS were detected in various measures, such as changes in the MEP amplitude and EEG power. The key differences in the present study are the larger stimulation intensity and the larger number of frequencies. In [7], the stimulation intensity was limited to $400 \mu \mathrm{A}$. However, this is about the level of the threshold intensity for the optimal stimulation frequency in our current study (see Figure 2). Therefore, it is likely that positive effects will be observed in other cortical areas when higher stimulation intensity is applied.

Ongoing rhythmic activities in the brain have been known about for many years. Recent studies began finding roles for such activities in attention and sensory awareness [18]. For example, it has recently been shown that fluctuation of ongoing alpha oscillation is related to excitability of the cortex and accounts for trial-by-trial variability of perception to a constant stimulus [38]. Our present technique offers the possibility of directly modifying ongoing activities in given frequency bands to influence behavioral responses by entraining or disrupting such activities.

\section{Supplemental Data}

Supplemental Data include Supplemental Experimental Procedures and can be found with this paper online at http://www.current-biology.com/ supplemental/S0960-9822(08)01396-1.

\section{Acknowledgments}

R.K. was supported by the Human Frontier Science Foundation. Development of tACS was initiated by an unrestricted grant by the Rose Foundation for L.C. and W.P. and was further funded by the Bernstein Center for Computational Neuroscience Göttingen (BMBF 01GQ0432, to A.A. and W.P.). The work at University College London was funded by the UK Medical Research Council (G0700929).

Received: August 15, 2008

Revised: September 10, 2008

Accepted: October 3, 2008

Published online: November 20, 2008

\section{References}

1. Walsh, V., and Pascual-Leone, A. (2003). Transcranial Magnetic Stimulation: A Neurochronometrics of Mind (Cambridge: MIT Press).

2. Wasserman, E., et al. (2008). The Oxford Handbook of Magnetic Brain Stimulation (Oxford: Oxford University Press).

3. Nitsche, M.A., and Paulus, W. (2000). Excitability changes induced in the human motor cortex by weak transcranial direct current stimulation. J. Physiol. 527, 633-639.
4. Priori, A., et al. (1998). Polarization of the human motor cortex through the scalp. Neuroreport 9, 2257-2260.

5. Nitsche, M.A., and Paulus, W. (2001). Sustained excitability elevations induced by transcranial DC motor cortex stimulation in humans. Neurology 57, 1899-1901.

6. Wassermann, E.M., and Grafman, J. (2005). Recharging cognition with DC brain polarization. Trends Cogn. Sci. 9, 503-505.

7. Antal, A., Boros, K., Poreisz, C., Chaieb, L., Terney, D., and Paulus, W. (2008). Comparatively weak after-effects of transcranial alternating current stimulation (tACS) on cortical excitability in humans. Brain Stimulation 1, 97-105.

8. Buzsaki, G. (2006). Rhythms of the Brain (Oxford: Oxford University Press).

9. Engel, A.K., Fries, P., and Singer, W. (2001). Dynamic predictions: oscillations and synchrony in top-down processing. Nat. Rev. Neurosci. 2, 704-716.

10. Womelsdorf, T., et al. (2007). Modulation of neuronal interactions through neuronal synchronization. Science 316, 1609-1612.

11. Womelsdorf, T., Fries, P., Mitra, P.P., and Desimone, R. (2006). Gamma band synchronization in visual cortex predicts speed of change detection. Nature 439, 733-736.

12. Marshall, L., Helgadottir, H., Mölle, M., and Born, J. (2006). Boosting slow oscillations during sleep potentiates memory. Nature 444,610 613.

13. Antal, A., et al. (2003). Manipulation of phosphene thresholds by transcranial direct current stimulation in man. Exp. Brain Res. 150, 375-378.

14. Antal, A., et al. (2004a). Excitability changes induced in the human primary visual cortex by transcranial direct current stimulation: direct electrophysiologicl evidence. Invest. Ophthalmol. Vis. Sci. 45, 702-707.

15. Berger, H. (1933). Über das Elektrenkephalogramm des Menschen. Arch F Psychiat 98, 231-254.

16. Adrian, E.D., and Matthews, B.H.C. (1934). The Berger rhythm: potential changes from the occipital lobes in man. Brain 57, 355-385.

17. Jasper, H.H. (1936). Cortical excitatory state and variability in human brain rhythms. Science 83, 259-260.

18. Palva, S., and Palva, J.M. (2007). New vistas for alpha-frequency band oscillations. Trends Neurosci. 30, 150-158.

19. Nitsche, M.A., et al. (2007). Shaping the effects of transcranial direct current stimulation of the human motor cortex. J. Neurophysiol. 97, 3109-3117.

20. Pirenne, M.H. (1962) in The Eye, (vol. 2) eds Davson, H. (Academic Press, London), Chapter 5: Dark Adaptation and Night Vision.

21. Boroojerdi, B., et al. (2000). Enhanced excitability of the human visual cortex induced by short-term light deprivation. Cereb. Cortex 10, 529534.

22. Tyrell, R.A., and Owens, D.A. (1998). A rapid technique to assess the resting states of the eye and other threshold phenomena: the modified binary search (MOBS). Behav. Res. Methods Instrum. Comput. 20, 137141.

23. Tootell, R.B.H., Hadjikhani, N.K., Mendola, J.D., Marrette, S., and Dale, A.M. (1998). Trends Cogn. Sci. 2, 174-183.

24. Sereno, M.I., et al. (1995). Borders of multiple visual areas in humans revealed by functional magnetic resonance imaging. Science 268 , 889-893.

25. Kammer, T. (1999). Phosphenes and transient scotomas induced by magnetic stimulation of the occipital lobe: their topographic relationship. Neuropsychologica 36, 191-198.

26. Baumann, S.B., Wozny, D.R., Kelly, S.K., and Meno, F.M. (1997). The electrical conductivity of human cerebrospinal fluid at body temperature. IEEE Trans. Biomed. Eng. 44, 220-223.

27. Polk, C., and Postow, E. (1996). Biological effects of electromagnetic fields, Second Edition (Boca Raton, FL: CRC Press), pp. 67.

28. Latikka, J., Kurne, T., and Eskola, H. (2001). Conductivity of living intracranial tissues. Phys. Med. Biol. 46, 1611-1616.

29. Brindley, G.S., and Lewin, W.S. (1968). The sensations produced by electrical stimulation of the visual cortex. J. Physiol. 196, 479-493.

30. Brindley, G.S. (1982). Visual prostheses. Hum. Neurobiol. 1, 281-283.

31. Dobelle, W.H., and Mladejovsky, M.G. (1974). Phosphenes produced by electrical stimulation of human occipital cortex, and their application to the development of a prosthesis for the blind. J. Physiol. 243, 553-576.

32. Dobelle, W.H., Mladejovsky, M.G., Evans, J.K., Roberts, T., and Girvin, J.P. (1976). 'Braille' reading by a blind volunteer by visual cortex stimulation. Nature 259, 111-112. 
33. Evans, J.R., Gordon, J., Abramov, I., Mladejovsky, M.G., and Dobelle, W.M. (1979). Brightness of phosphenes elicited by electrical stimulation of human visual cortex. Sen. Proc. 3, 82-94.

34. Hines, M. (1940). Movements elicited from precentral gyrus of adult chimpanzees by stimulation with sine wave currents. J. Neurophysiol. 3, 442-466.

35. Merton, P.A., and Morton, H.B. (1980). Stimulation of the cerebral cortex in the intact human subject. Nature 285, 227.

36. Merton, P.A., Hill, D.K., Marsden, C.D., and Morton, H.B. (1982). Scope of a technique for electrical stimulation of human brain, spinal cord and muscle. Lancet 320, 597-600.

37. Cohen, L.G., and Hallet, M. (1988). Methodology for non-invasive mapping of human motor cortex with electrical stimulation. Electroencephalogr. Clin. Neurophysiol. 69, 403-411.

38. Romei, V., Brodbeck, V., Michel, C., Amedi, A., Pascual-Leone, A., and Thut, G. (2008). Spontaneous fluctuations in posterior alpha-band EEG activity reflect variability in excitability of human visual areas. Cereb. Cortex 18, 2010-2018. 


\subsection{Pergolide increases the efficacy of cathodal direct current stimulation to reduce the amplitude of laser-evoked potentials in humans}

Terney D, Bergmann I, Poreisz C, Chaieb L, Boros K, Nitsche MA, Paulus W, Antal A. J Pain Symptom Manage. 2008; 36(1):79-91.

Transcranial direct current stimulation (tDCS) has been shown to be effective in inducing sustained excitability changes in the human cortex. In this study we aimed to target the enhancement of D2 receptor potentiation by pergolide, shown to consolidate tDCS induced decreases in excitability, to investigate the effect of tDCS on laserinduced pain perception. The amplitude of the $\mathrm{N} 2$ component, an indicator of pain perception in EEG- power, was significantly reduced following combined treatment with cathodal tDCS and pergolide. Pergolide also prolonged this anti- nociceptive effect for 24 hours poststimulation. This study demonstrates the potential application of tDCS within a clinical setting for the treatment of pain syndromes. 


\title{
Original Article
}

\section{Pergolide Increases the Efficacy of Cathodal Direct Current Stimulation to Reduce the Amplitude of Laser-Evoked Potentials in Humans}

Daniella Terney, MD, Inga Bergmann, Csaba Poreisz, MD, Leila Chaieb, MSc, Klára Boros, MD, Michael Andreas Nitsche, MD, Walter Paulus, MD, and Andrea Antal, PhD

Department of Clinical Neurophysiology (D.T., I.B., C.P, L.C. K.B., M.A.N., W.P., A.A.), Georg-August University, Göttingen, Germany; and Department of Neurology (D.T.), University of Szeged, Szeged, Hungary

\begin{abstract}
Transcranial direct current stimulation (tDCS) was recently reintroduced as a tool for inducing relatively long-lasting changes in cortical excitability in focal brain regions. Anodal stimulation over the primary motor cortex enhances cortical excitability, whereas cathodal stimulation decreases it. Prior studies have shown that enhancement of D2 receptor activity by pergolide consolidates $t D C S$-generated excitability diminution for up to 24 hours and that cathodal stimulation of the primary motor cortex diminishes experimentally induced pain sensation and reduces the N2-P2 amplitude of laser-evoked potentials immediately poststimulation. In the present study, we investigated the effect of pergolide and cathodal tDCS over the primary motor cortex on laser-evoked potentials and acute pain perception induced with a Tm:YAG laser in a double-blind, randomized, placebo-controlled, crossover study. The amplitude changes of laser-evoked potentials and subjective pain rating scores of 12 healthy subjects were analyzed prior to and following 15 minutes cathodal tDCS combined with pergolide or placebo intake at five different time points. Our results indicate that the amplitude of the N2 component was significantly reduced following cathodal tDCS for up to two hours. Additionally, pergolide prolonged the effect of the cathodal tDCS for up to 24 hours, and a significantly lowered pain sensation was observed for up to 40 minutes. Our study is a further step toward clinical application of cathodal tDCS over the primary motor cortex using pharmacological intervention to prolong the excitability-diminishing effect on pain perception for up to 24 hours poststimulation. Furthermore, it demonstrates the potential for repetitive daily stimulation therapy for pain patients. J Pain Symptom Manage
\end{abstract}

This study was performed within the "Kompetenznetz Schmerz" (FKZ: 01EM0117), funded by the German Ministry of Research and Education.

Address correspondence to: Daniella Terney, MD, Department of Clinical Neurophysiology, Georg-

(C) 2008 U.S. Cancer Pain Relief Committee Published by Elsevier Inc. All rights reserved.
August University, Robert Koch Strasse 40, 37075 Göttingen, Germany. E-mail: daniellaterney@ yahoo.co.uk

Accepted for publication: August 8, 2007.

0885-3924/08/\$-see front matter doi:10.1016/j.jpainsymman.2007.08.014 
2008;36:79-91. (C) 2008 U.S. Cancer Pain Relief Committee. Published by Elsevier Inc. All rights reserved.

Key Words

Pain, tDCS, pergolide, LEP, motor cortex, human

\section{Introduction}

In recent years, invasive stimulation of the primary motor cortex (M1) for the treatment of certain kinds of pain has attracted much interest. The first widely accepted clinical method for alleviating pain using cortical stimulation was epidural electrical motor cortex stimulation. $^{1,2}$ Recently, the most frequently investigated noninvasive method so far is repetitive transcranial magnetic stimulation (rTMS). Studies of transcranial magnetic stimulation in experimental and acute pain sensations have produced encouraging outcomes. ${ }^{3,4}$ In spite of the beneficial effects of rTMS, a new method, transcranial direct current stimulation (tDCS) has been favored in recent editorials. ${ }^{5,6}$

Major advantages of tDCS as a tool for inducing long-lasting changes of cortical excitability and activity in focal brain regions is that it acts reversibly, painlessly, and safely. ${ }^{7-10}$ Primarily, it causes polarity-dependent shifts of the resting membrane potentials and consequently changes the firing rates of neurons under the electrodes, neuronal projections and subsequent connected cortical areas. ${ }^{11-13}$ Generally, anodal stimulation over the M1 has been found to enhance cortical excitability, whereas cathodal stimulation decreases it. ${ }^{78}$ Although in humans the modulatory effect of tDCS had first been demonstrated on the motor system, it influences visual, somatosensory and prefrontal functions as well. ${ }^{14-16}$ In a recent study, it was shown that enhancement of D2, and to a much lesser degree, of D1 receptor activity by pergolide consolidated cathodal tDCS-generated excitability diminution for up to 24 hours. ${ }^{17}$

Our first sham-controlled studies demonstrated that cathodal stimulation of the M1 diminishes experimentally induced pain sensation, and in parallel reduces the N2-P2 amplitude of laser-evoked potentials (LEPs) immediately after the end of stimulation. ${ }^{18}$ The aim of the present study was to investigate the effect of combined cathodal stimulation and pergolide treatment on LEPs and related pain perception in a double-blind, randomized, placebo-controlled, crossover study, with the clear intention of proving the already known inhibitory prolonging effect of pergolide $^{17}$ on pain perception. Here, amplitude changes of the N1, N2, and P2 of LEPs and subjective pain rating scores of 12 healthy subjects were analyzed prior to and following 15 minutes of cathodal tDCS, and following pergolide or placebo treatment at five different time points (before, $0 \mathrm{~min}, 40 \mathrm{~min}$, two hours, 24 hours).

\section{Methods}

\section{Subjects}

Fifteen healthy volunteers (aged between 20 and 31 years) were informed about all aspects of the experiments and all gave informed consent. Two participants chose not to continue the experiment after the first trials, and one subject was excluded as LEPs could not be identified reliably; 12 of the subjects (five male, seven female) were included in the study. All of the subjects underwent pergolide and placebo medication treatment. Additionally, seven subjects (three male, four female) participated in a control session in which no tDCS and drug treatment were introduced. We conform to the Declaration of Helsinki and the experimental protocol was approved by the Ethics Committee of the University of Göttingen. None of the subjects suffered from any neurological and psychological disorders, and none had metallic implants/ implanted electric devices, nor took any medication regularly.

\section{Pharmacological Interventions}

Pergolide $0.025 \mathrm{mg}$ combined with $10 \mathrm{mg}$ domperidone to avoid side effects (e.g., vomiting induced by the medication) or equivalent 
placebo (glucose) was taken by the subjects orally two hours prior to the start of the experiments. By this means, the drug induces a stable plasma level ${ }^{19}$ and produces prominent effects in the central nervous system. ${ }^{17,20,21}$ To avoid cumulative drug effects, each experimental session was separated by at least one week. Subjects and the investigator conducting the experiment were blinded as to the respective pharmacological condition.

\section{$t D C S$}

tDCS was delivered by a battery-driven constant current stimulator (Eldith NeuroConn GmbH, Ilmenau, Germany) using a pair of rubber electrodes in a $5 \times 7 \mathrm{~cm}$ water-soaked synthetic sponge. The cathode was placed over the representational field of the right abductor digiti minimi as identified by transcranial magnetic stimulation (Dantec S.A., Skovlunde, Denmark), whereas the other electrode (reference) was situated contralaterally above the right eyebrow. The electrodes were oriented approximately parallel to the central sulcus and the eyebrow. This montage has been proven to be the most effective for modulating motor cortex excitability. ${ }^{7}$ The cathodal stimulation refers to the polarity of the electrode above the M1. The current was applied for 15 minutes with an intensity of $1.0 \mathrm{~mA}$.

\section{Laser Stimulation}

A Tm:YAG laser system (WaveLight Laser Technologie AG, Erlangen, Germany) was used for the pain stimulation. The thulium laser emits near-infrared radiation (wavelength 2,000 nm, pulse duration 1 millisecond, laser beam diameter $7 \mathrm{~mm}$ ) with a penetration depth of $360 \mu \mathrm{m}$ into the human skin and allows a precise restriction of the emitted heat energy to the termination area of primary nociceptive afferents without affecting the subcutaneous tissue $e^{22,23}$ The distal handpiece of the laser was positioned $30 \mathrm{~cm}$ from the radial part of the dorsal surface of the hand. Skin temperature of the stimulated area was checked prior to every switch between hands, and corrected with a heating lamp if it fell below $35^{\circ} \mathrm{C}$. We stimulated slightly different spots in a square $(5 \times 5 \mathrm{~cm})$ for each measurement to reduce receptor fatigue or sensitization by skin overheating. ${ }^{23}$ In both experiments, the right hand was stimulated first in half of the cases and the left hand was stimulated first in the other half. This approach, used as increased response toward novel stimuli, has been described in evoked potential studies of other sensory modalities. ${ }^{24-27}$

At the beginning of each condition the pain threshold of both hands was determined by applying laser stimuli from $200 \mathrm{~mJ}$ in $50 \mathrm{~mJ}$ steps. During EEG recording, we delivered 40 laser pulses to each hand before and after tDCS with 1.5-1.6 times of threshold intensity. The interstimulus interval of the stimulation ranged from eight to 15 seconds. During each condition, the intensity of the laser stimulation was kept constant as determined prior to tDCS, enabling a clear comparison between results.

\section{Psychophysical Evaluation}

We used the numeric analog score to assess the subjective intensity of pain. The subjects were instructed to pay attention to the laser stimuli and to rate the perceived pain verbally (warm: 1, painful: from 2 (smallest) to 10 (most intense pain)) about 2-3 seconds after each stimulation. ${ }^{28}$ The ears of the subjects were plugged during the measurements to avoid auditory artifacts produced by the laser stimulation.

\section{Electrophysiological Recordings}

The electroencephalogram was recorded using a five-channel montage as described by Treede et al. ${ }^{23}$ This montage has been used in numerous experimental and clinical LEP studies as it enables the easy identification of late LEP components. Electroencephalogram was recorded with gold disc electrodes from the Fz, Cz, Pz, T3, T4 (vs. linked mastoids) according to the international $10 / 20$ system. The ground electrode was positioned on the forehead. The impedance was kept below 5 $\mathrm{kOhm}$. Data were collected with a sampling rate of $1,000 \mathrm{~Hz}$ by the BrainAmp system (Brain Products GmbH, Munich, Germany) and were analyzed offline. A $0.5 \mathrm{~Hz}$ low cutoff and a $30 \mathrm{~Hz}$ high cutoff filter were used. After semiautomatic artifact detection $(150 \mu \mathrm{V}$ amplitude criterion), all epochs were visually inspected as well, and those containing eye blinks or muscle movement artifacts were excluded. All recordings consisted of at least 35 
artifact-free epochs. Baseline correction was performed on the basis of the 100 millisecond prestimulus interval. Using semiautomatic peak detection, we investigated different LEP components. The earliest component is a negativity N1 (peaking around 140-170 milliseconds), using T3 and T4 channels vs. Fz. The $\mathrm{N} 1$ component is followed by the late N2-P2 complex (N2: peaking around 160-220 milliseconds, P2: peaking around 300-360 milliseconds) in the midline $(\mathrm{Fz}, \mathrm{Cz}, \mathrm{Pz})$ leads, using linked mastoid reference.

\section{Experimental Procedures}

The experiments were conducted in a repeated measurement design using a randomized order, with a break of at least one week between each session. Pergolide $0.025 \mathrm{mg}$ or equivalent placebo (glucose) was taken by the subjects orally two hours before the start of the experiments. The subjects were seated in a reclining chair. First, the left motor-cortical representational field of the right abductor digiti minimi was identified using transcranial magnetic stimulation. At the beginning of each condition, the pain threshold of both hands was determined by applying laser stimuli from $200 \mathrm{~mJ}$ in $50 \mathrm{~mJ}$ steps. During electroencephalogram recording, we delivered 40 laser pulses to each hand before tDCS with 1.5-1.6 times of threshold intensity. Afterward, cathodal tDCS was performed for 15 minutes, followed by 40 laser pulses to each hand immediately after the stimulation, 40 minutes, two hours, and 24 hours later (Fig. 1).

Because our previous study has shown that sham stimulation has no significant effect on pain sensation, ${ }^{18}$ no sham stimulation was used as an additional condition. However, we aimed to examine the normal habituation process. Seven subjects, chosen among the ones participating in the previous experiment, underwent the same protocol described previously, in which no tDCS and drug condition were introduced.

\section{Data Analysis}

Because the size of the amplitudes differed across subjects, normalization of the data was necessary. We divided the "after" tDCSconditions by the value of the "before" condition. As a consequence of the bilateral representation of pain ${ }^{29,30}$ and the lack of significant differences $(P>0.05)$ between the
LEP amplitudes of the two sides, the data were not analyzed separately. Averaged numeric analog score values for N1, N2, and P2 amplitudes from each set of 40 trials were individually averaged and entered into a repeated-measures analysis of variance (ANOVA) (2 medications CONDITION [pergolide, placebo] $\times 4$ TIME [after $0 \mathrm{~min} /$ before, after $40 \mathrm{~min} /$ before, after 2 hours/before, after 24 hours/before]). To compare the control results with the under medication conditions, all results of the seven subjects (3 CONDITION [pergolide, placebo, control] $\times 4$ TIME) were also individually averaged and entered into a repeated-measures ANOVA. In addition, a one-way ANOVA was performed separately for each condition to show the effectiveness of direct current (DC) stimulation. We considered a psychophysical or an electrophysiological change if the CONDITION $\times$ TIME interaction was significant or if the one-way ANOVA revealed significant difference between time points. Post-hoc analysis was done using a Fischer LSD test.

\section{Results}

\section{Psychophysics}

The intensity of the laser stimulation was $21.32 \mathrm{~mJ} / \mathrm{mm}^{2}$ for pergolide medication (range, $19.5-23.4 \mathrm{~mJ} / \mathrm{mm}^{2}$ ), $21.177 \mathrm{~mJ} / \mathrm{mm}^{2}$ for placebo medication (range, 19.5-23.4 mJ/ $\mathrm{mm}^{2}$ ), and $21.32 \mathrm{~mJ} / \mathrm{mm}^{2}$ (range, 19.5-22.1 $\mathrm{mJ} / \mathrm{mm}^{2}$ ) for the control measurements (without tDCS and medication).

Concerning the pain perception scale, the ANOVA revealed no main effect of CONDITION $[F(1,23)=2.38, P=0.136]$, but the effect of TIME was significant $[F(3,69)=10.89$, $P<0.005]$. The CONDITION $\times$ TIME interaction was not significant $[F(3,69)=0.50, P=$ $0.680]$. If we compared the control results with the medication conditions, there was no main effect of CONDITION $[F(2,26)=1.56, P=$ $0.229]$, but the effect of TIME $[F(3,39)=7.47$, $P<0.005$ ] was significant (Fig. 2). There was no significant difference in the CONDITION $\times$ TIME interaction $[F(6,78)=0.80, P=0.570]$.

One-way ANOVA revealed significant effect of TIME in the case of pergolide medication $[F(4,92)=6.06, P<0.005]$. The post-hoc analysis showed a significant difference between the before and after conditions $(P<0.05)$. In the 


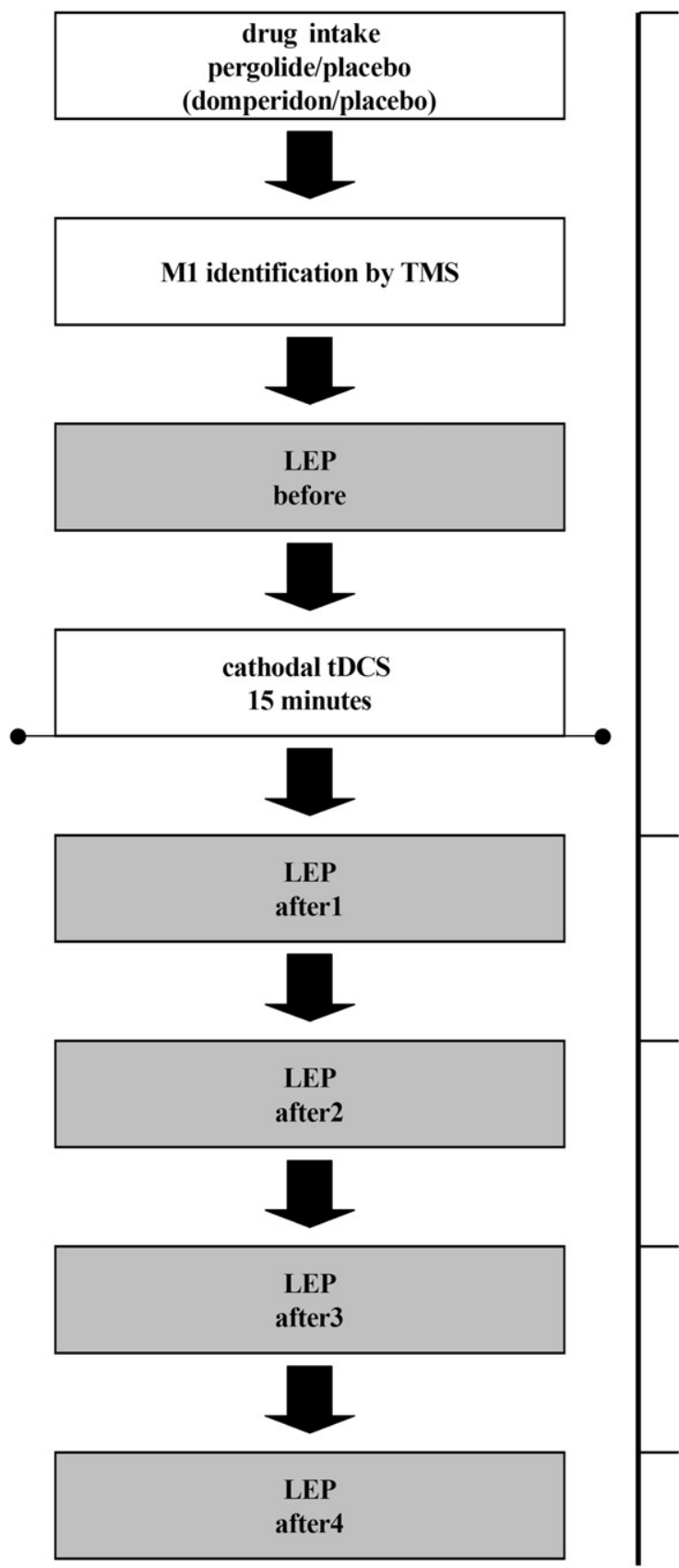

\section{5 hours \\ before tDCS}

0 minute

following after tDCS

40 minutes

following after tDCS

2 hours

following after tDCS

24 hours

following after tDCS

Fig. 1. Experimental procedure: Pergolide or an equivalent placebo drug was taken by all subjects orally two hours before the start of the experiments (2.5 hours before tDCS). First, the left motor-cortical representational field of the right abductor digiti minimi was identified using TMS. During electroencephalogram recording, we delivered 40 laser pulses to each hand before tDCS. Afterward, cathodal tDCS was performed for 15 minutes, followed by 40 laser pulses to each hand immediately after the stimulation, 40 minutes, two hours, and 24 hours later.

case of placebo medication, the effect of TIME was significant $[F(4,92)=4.54, P=0.002]$ and a significant difference was revealed between the after and all the other time points $(P<0.05)$. In the case of the control experiment, one-way ANOVA revealed no significant effect of TIME $[F(4,52)=0.94, P=0.446]$.

The means and standard deviations of numeric analog score values for both hands and for all conditions are shown in Table 1. 


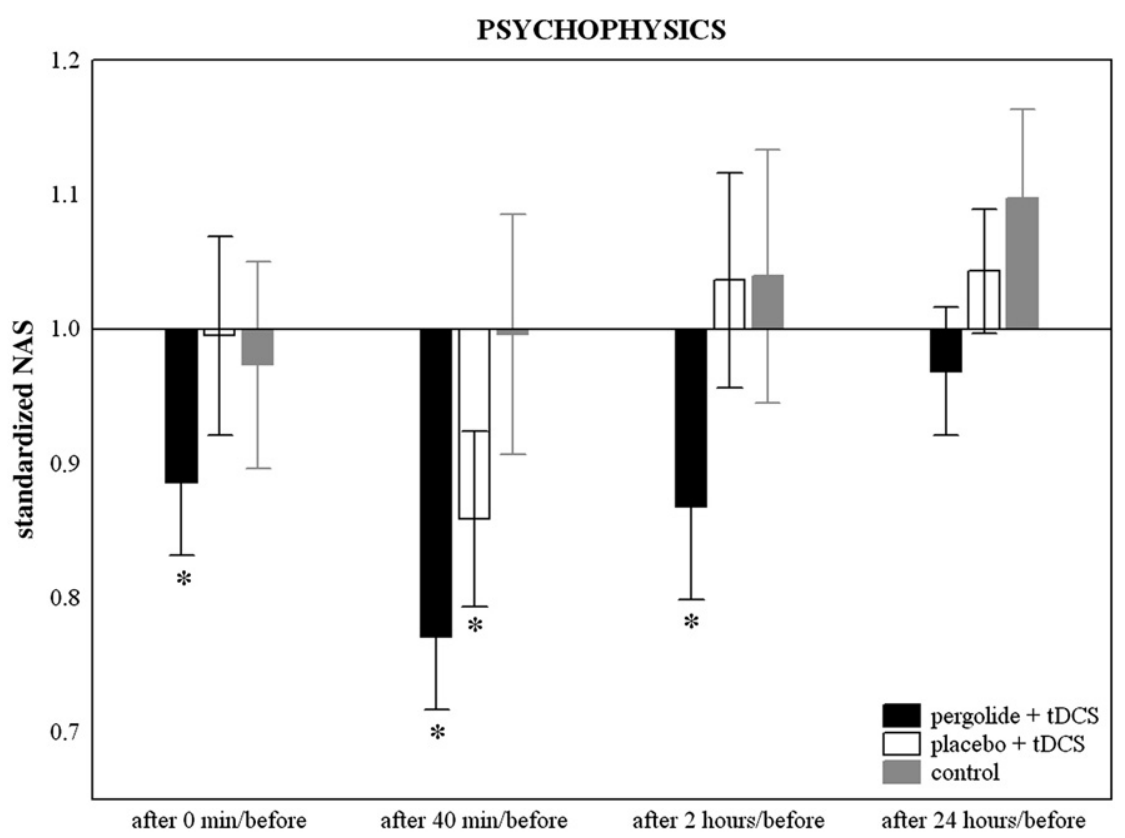

Fig. 2. The differences between numeric analog score results at four time points (standardized data by calculating the after $1-4$ /before ratio), for the two medication conditions (pergolide, placebo) and control experiment for both hands with laser stimulation. The standardized numeric analog score results show either an increase in pain sensation or a decline, relative to one. Following cathodal tDCS, the pain sensation was lowered up to 40 minutes. The asterisks indicate significant differences between the different time points.

\section{Electrophysiology}

The laser stimulation induced a pricking pain and a biphasic N2-P2 component was clearly identified in all LEP measures of all 12 subjects (Fig. 3 ).

N1 Component. In the case of the N1 component, there was no main effect of CONDITION $[F(1,23)=0.437, P=0.515]$ or TIME $[F(3,69)=$ $0.14, P=0.937]$ at the T3 electrode position. There was no significant CONDITION $\times$ TIME interaction $[F(3,69)=0.28, P=0.840]$. At the $\mathrm{T} 4$ electrode position, there was no main effect of CONDITION $[F(1,23)=0.116, P=0.325]$ or TIME $[F(3,69)=0.24, P=0.654]$. There was no significant CONDITION $\times$ TIME interaction
$[F(3,69)=0.38, \quad P=0.213] . \quad$ In the control experiment, there was no main effect of CONDITION, or TIME. There was no significant difference in the CONDITION $\times$ TIME interaction $(P>0.005)$.

N2 Component. In the case of the N2 component, there was no main effect of CONDITION $[F(1,23)=0.12, P=0.737]$, but the effect of TIME was significant $[F(3,69)=7.44, P<0.005]$ at the Fz electrode position. There was no significant difference in the CONDITION $\times$ TIME interaction $[F(3,69)=1.369, P=0.259]$. At the $\mathrm{Cz}$ electrode position, there was no main effect of CONDITION $[F(1,23)=0.91, \quad P=0.349]$, but the effect of TIME was significant

Table 1

Averaged Numeric Analog Score Values and Standard Deviations from Each Set of 40 Trials

\begin{tabular}{lllcccc}
\hline Condition & Side & Before & After1 & After2 & After3 & After4 \\
\hline Pergolide + tDCS, $n=12$ & Left & $4.91 \pm 2.02$ & $4.37 \pm 1.98$ & $4.06 \pm 2.04$ & $4.25 \pm 2.27$ & $4.89 \pm 2.09$ \\
& Right & $5.05 \pm 1.77$ & $4.30 \pm 2.03$ & $3.66 \pm 2.09$ & $4.50 \pm 2.32$ & $4.65 \pm 1.79$ \\
Placebo + tDCS, $n=12$ & Left & $4.05 \pm 1.34$ & $4.14 \pm 1.41$ & $3.55 \pm 1.37$ & $4.14 \pm 1.72$ & $4.36 \pm 1.48$ \\
& Right & $4.39 \pm 1.39$ & $3.98 \pm 1.66$ & $3.46 \pm 1.34$ & $4.41 \pm 1.91$ & $4.27 \pm 1.32$ \\
Control, $n=7$ & Left & $3.69 \pm 1.76$ & $3.31 \pm 1.56$ & $3.43 \pm 2.15$ & $3.61 \pm 1.79$ & $4.02 \pm 1.40$ \\
& Right & $3.28 \pm 1.15$ & $3.19 \pm 1.32$ & $3.59 \pm 1.88$ & $3.35 \pm 1.90$ & $3.49 \pm 1.55$ \\
\hline
\end{tabular}

Averaged numeric analog score values from each set of 40 trials for the left and right sides separately and all conditions before and after tDCS. 
before

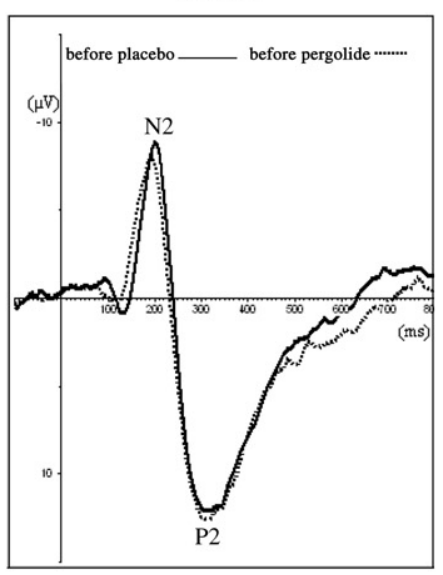

after $40 \mathrm{~min}$

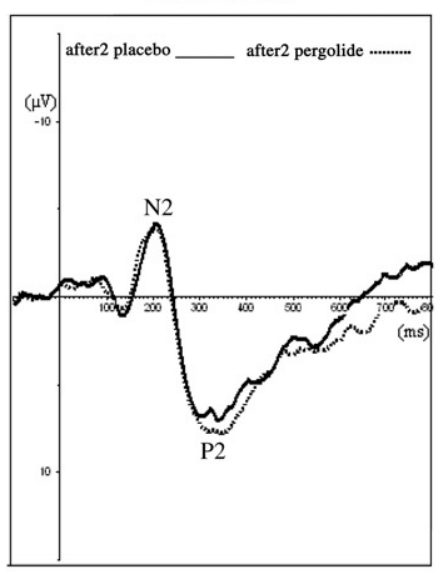

after $0 \mathrm{~min}$

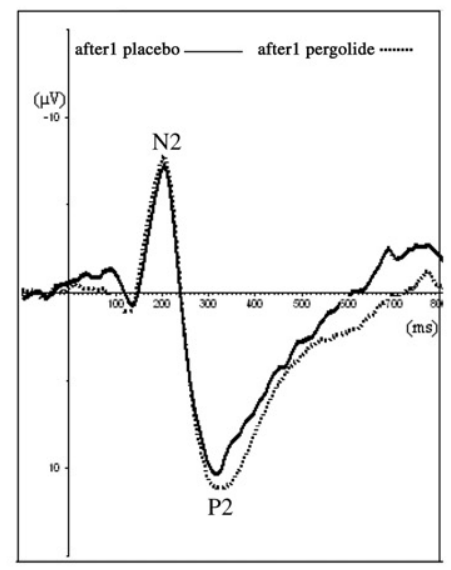

after 2 hours

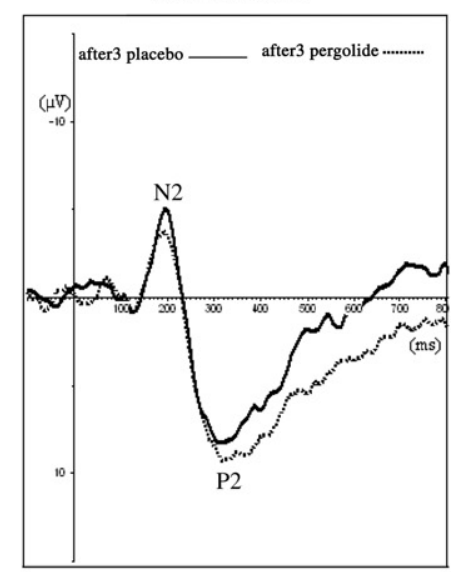

after 24 hours

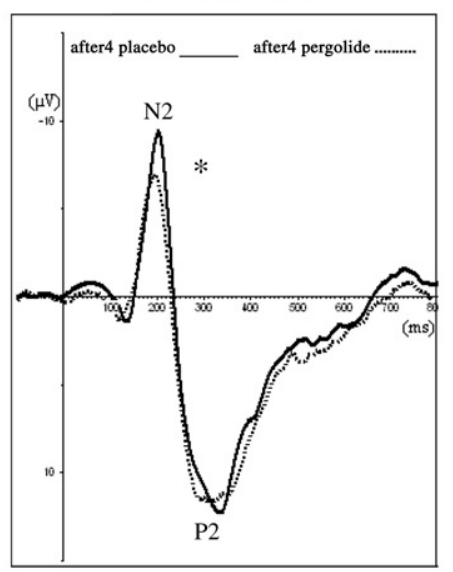

Fig. 3. Grand averages of LEPs obtained by both hand laser stimulation for the Cz recording electrode. The solid line shows LEPs for placebo medication combined with tDCS and the intermittent line for pergolide medication combined with tDCS at five different time points (before, after tDCS $0 \mathrm{~min}$, after tDCS $40 \mathrm{~min}$, after tDCS two hours, after tDCS 24 hours). Note that 24 hours following tDCS a greater amplitude reduction of the N2 component and N2P2 peak-to-peak amplitude for pergolide medication is observed when compared to placebo medication. The asterisk indicates a significant difference between the pergolide and placebo conditions. 
$[F(3,69)=5.65, \quad P=0.001]$. We also found a significant CONDITION $\times$ TIME interaction $[F(3,69)=3.67, P=0.016]$. According to the post hoc analysis, pergolide medication, significantly decreased the amplitudes of the N2 component, compared to the placebo medication, at the 24-hour time point $(P=0.006)$. However, there were no significant differences between the other time points when compared with the pergolide and placebo medication $(P>0.05)$. At the $\mathrm{Pz}$ electrode position, there was no main effect of CONDITION $[F(1,23)=$ $1.93, P=0.178]$, but the effect of TIME was significant $[F(3,69)=6.82, P<0.005]$. There was no significant CONDITION $\times$ TIME interaction $[F(3,69)=2.16, P=0.101]$.

To compare the control results to the under medication conditions, the results of the seven subjects were also individually averaged and entered into a repeated-measures ANOVA for all electrode positions. Although the effect of TIME was significant at all electrode positions $(P<0.05)$, neither the effect of CONDITION nor the CONDITION $\times$ TIME interaction were significant $(P>0.05)$.

One-way ANOVA revealed significant effect of TIME in the case of pergolide medication $[F(3,69)=3.65, P=0.017]$. The post hoc analysis showed a significant difference between the before and after conditions $(P=0.002)$. In the case of placebo medication, the effect of TIME was significant $[F(3,69)=6.60, P<0.005]$ and a significant difference was revealed between the before and after, and after and after, conditions $(P<0.05)$. In the case of the control experiment, one-way ANOVA revealed no significant effect of TIME $[F(3,39)=1.57, P=0.211]$.

P2 Component. Although the effect of TIME was significant $(P<0.05)$ in the case of the $\mathrm{P} 2$ component, there was no main effect of CONDITION, nor a significant CONDITION $\times$ TIME interaction at all electrode positions $(P>0.05)$. If we compared the control results with the under medication conditions, the effect of TIME was significant at all electrode positions, but neither the effect of CONDITION, nor the CONDITION $\times$ TIME interaction were significant $(P>0.05)$.

The means and standard deviations for the right and left hand separately, and under all conditions, are shown in Tables 2 and 3.

\section{Discussion}

tDCS modifies the excitability of the stimulated cortical area in a polarity-dependent way $^{7,8}$ and simultaneously causes perceptual changes. $^{31,32}$ In the present study, we explored the effects of this noninvasive brain stimulation technique on subjective acute pain perception and its electrophysiological correlates. Our results confirm that 15 minutes of cathodal tDCS over the primary motor cortex significantly reduced the amplitude of the N2 component, and the changes of the electrophysiological parameter remained stable for up to two hours after stimulation when compared to the control experiment (Fig. 4). Furthermore, the subjective pain sensation was lowered after cathodal tDCS for up to $40 \mathrm{~min}$ utes (Fig. 2).

Recording LEPs is a widely accepted method for examining the neuronal correlates of pain perception temporally and spatially in human subjects. ${ }^{22,23}$ The earliest cortical LEP component is a negativity (N1, peaking around 140-170 milliseconds). According to its scalp topography (maximum near T3 and T4), it is

Table 2

Mean Values and Standard Deviations of the LEP Parameters for the Right Hand

\begin{tabular}{|c|c|c|c|c|c|c|}
\hline Condition & Component & Before tDCS & $\begin{array}{l}\text { After } 1 \mathrm{tDCS} \\
(0 \mathrm{~min})\end{array}$ & $\begin{array}{l}\text { After } 2 \text { tDCS } \\
(40 \mathrm{~min})\end{array}$ & $\begin{array}{l}\text { After3 tDCS } \\
\text { (2 hours) }\end{array}$ & $\begin{array}{l}\text { After4 tDCS } \\
\text { (24 hours) }\end{array}$ \\
\hline Pergolide + tDCS, $n=12$ & $\begin{array}{l}\text { N2 }(\mu \mathrm{V}) \\
\mathrm{P} 2(\mu \mathrm{V})\end{array}$ & $\begin{array}{r}-11.42 \pm 6.40 \\
14.87 \pm 5.54\end{array}$ & $\begin{array}{r}-10.09 \pm 5.55 \\
12.82 \pm 6.12\end{array}$ & $\begin{array}{r}-7.23 \pm 4.49 \\
10.9 \pm 6.14\end{array}$ & $\begin{array}{r}-7.32 \pm 5.97 \\
11.68 \pm 7.58\end{array}$ & $\begin{array}{r}-9.33 \pm 5.33 \\
13.55 \pm 5.90\end{array}$ \\
\hline Placebo $+\mathrm{tDCS}, n=12$ & $\begin{array}{l}\text { N2 }(\mu V) \\
\text { P2 }(\mu V)\end{array}$ & $\begin{array}{r}-11.04 \pm 5.82 \\
15.14 \pm 6.96\end{array}$ & $\begin{array}{r}-9.36 \pm 6.59 \\
12.27 \pm 7.11\end{array}$ & $\begin{array}{r}-6.78 \pm 4.23 \\
9.07 \pm 5.44\end{array}$ & $\begin{array}{r}-7.38 \pm 3.66 \\
11.53 \pm 5.23\end{array}$ & $\begin{array}{r}-11.39 \pm 5.32 \\
14.76 \pm 5.49\end{array}$ \\
\hline Control, $n=7$ & $\begin{array}{l}\text { N2 }(\mu \mathrm{V}) \\
\mathrm{P} 2(\mu \mathrm{V})\end{array}$ & $\begin{array}{r}-13.12 \pm 7.52 \\
12.94 \pm 3.51\end{array}$ & $\begin{array}{r}-11.83 \pm 7.92 \\
10.06 \pm 3.21\end{array}$ & $\begin{array}{r}-10.31 \pm 6.44 \\
8.79 \pm 5.25\end{array}$ & $\begin{array}{r}-8.67 \pm 5.42 \\
9.57 \pm 3.83\end{array}$ & $\begin{array}{r}-12.45 \pm 7.75 \\
11.32 \pm 2.05\end{array}$ \\
\hline
\end{tabular}

The table shows the mean values and standard deviations of the LEP parameters for all conditions at the Cz electrode positions obtained from all subjects for right hand before and after cathodal tDCS. 
Table 3

Mean Values and Standard Deviations of the LEP Parameters for the Left Hand

\begin{tabular}{|c|c|c|c|c|c|c|}
\hline Condition & Component & Before tDCS & $\begin{array}{l}\text { After } 1 \mathrm{tDCS} \\
\quad(0 \mathrm{~min})\end{array}$ & $\begin{array}{l}\text { After2 tDCS } \\
(40 \mathrm{~min})\end{array}$ & $\begin{array}{l}\text { After3 tDCS } \\
\text { ( } 2 \text { hours })\end{array}$ & $\begin{array}{l}\text { After4 tDCS } \\
\text { ( } 24 \text { hours) }\end{array}$ \\
\hline Pergolide + tDCS, $n=12$ & $\begin{array}{l}\text { N2 }(\mu \mathrm{V}) \\
\mathrm{P} 2(\mu \mathrm{V})\end{array}$ & $\begin{array}{r}-11.73 \pm 4.66 \\
15.22 \pm 6.27\end{array}$ & $\begin{array}{r}-9.98 \pm 5.52 \\
12.48 \pm 6.87\end{array}$ & $\begin{array}{r}-7.45 \pm 5.71 \\
10.38 \pm 6.40\end{array}$ & $\begin{array}{r}-5.83 \pm 7.01 \\
10.75 \pm 6.39\end{array}$ & $\begin{array}{r}-8.5 \pm 5.19 \\
14.22 \pm 7.03\end{array}$ \\
\hline Placebo $+\mathrm{tDCS}, n=12$ & $\begin{array}{l}\text { N2 }(\mu \mathrm{V}) \\
\mathrm{P} 2(\mu \mathrm{V})\end{array}$ & $\begin{array}{r}-11.57 \pm 6.96 \\
15.55 \pm 8.60\end{array}$ & $\begin{array}{r}-9.16 \pm 5.81 \\
11.83 \pm 6.61\end{array}$ & $\begin{array}{r}-5.56 \pm 4.15 \\
9.41 \pm 6.89\end{array}$ & $\begin{array}{r}-6.37 \pm 4.52 \\
11.61 \pm 8.02\end{array}$ & $\begin{array}{r}-11.52 \pm 6.30 \\
15.58 \pm 7.02\end{array}$ \\
\hline Control, $n=7$ & $\begin{array}{l}\text { N2 }(\mu \mathrm{V}) \\
\mathrm{P} 2(\mu \mathrm{V})\end{array}$ & $\begin{array}{r}-13.22 \pm 6.70 \\
11.95 \pm 3.52\end{array}$ & $\begin{array}{r}-10.68 \pm 7.76 \\
8.67 \pm 3.91\end{array}$ & $\begin{array}{r}-8.48 \pm 4.95 \\
9.46 \pm 3.85\end{array}$ & $\begin{array}{r}-9.15 \pm 5.93 \\
9.61 \pm 4.46\end{array}$ & $\begin{array}{r}-11.89 \pm 5.84 \\
12.45 \pm 2.46\end{array}$ \\
\hline
\end{tabular}

The table shows the mean values and standard deviations of the LEP parameters for all conditions at the Cz electrode positions obtained from all subjects for left hand before and after cathodal tDCS.

probably generated near the secondary somatosensory cortex in the fronto-parietal operculum. ${ }^{23}$ We did not find any significant change concerning the N1 amplitudes. Probably, this area could not be stimulated directly or the intensity of stimulation used was not sufficient to reflect any significant change.

The N1 component is followed by the late negative-positive complex (N2-P2) that can be most accurately recorded in the midline (Fz, Cz, Pz) leads. According to source localizing studies, the N2 component (peaking around 160-220 milliseconds) is generated both bilaterally in the operculoinsular region and partly in the anterior cingulate cortex (ACC). ${ }^{2}$ This component contributes to sensory-discriminatory aspects of pain. The P2 component (peaking around 300-360 milliseconds) arises mainly from the ACC and reflects endogenous, attentional-cognitive, ${ }^{33}$ and affective factors. ${ }^{23,34}$ The role of ACC in coding pain intensity is still under debate; however, there is increasing evidence to suggest that activity in some parts of the ACC

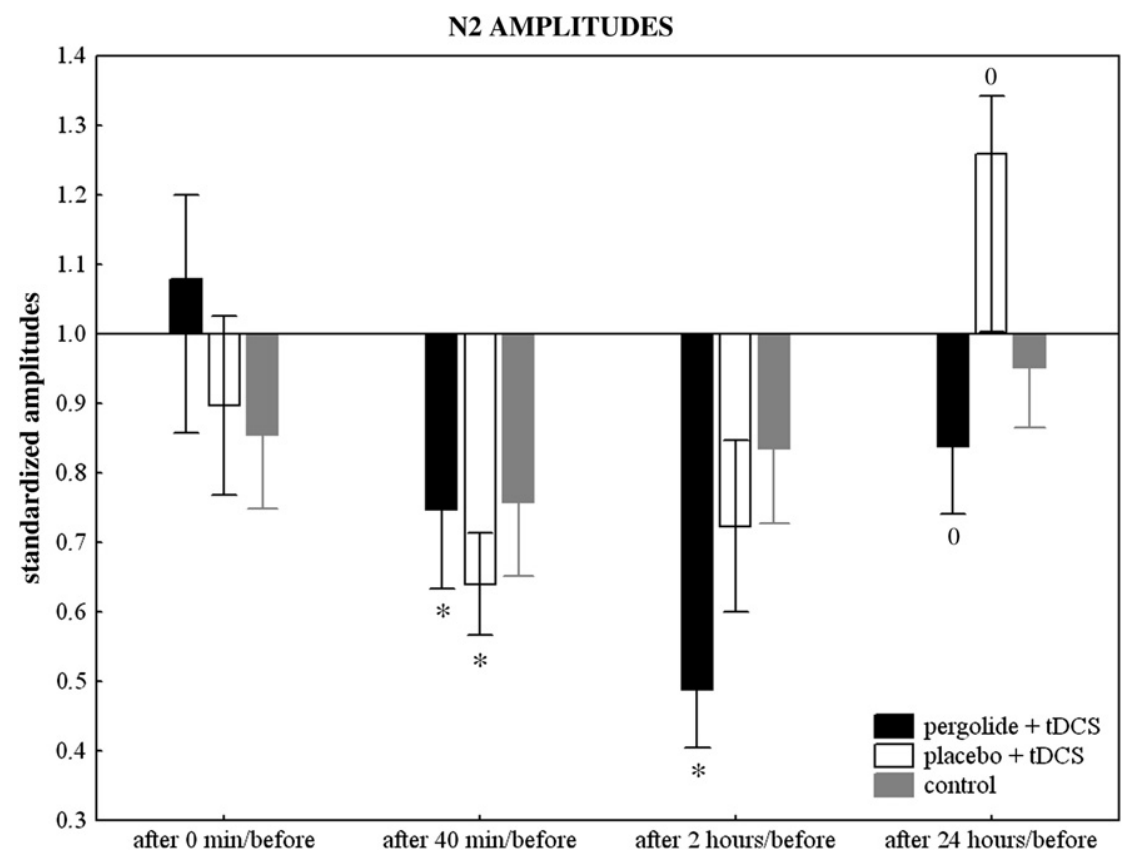

Fig. 4. The differences between mean N2 amplitude values at four time points (standardized data by calculating the after 1-4/before ratio), for the two medication conditions (pergolide, placebo) and control experiment for both hands laser stimulation at the $\mathrm{Cz}$ electrode. The standardized peak amplitudes show either an increase in the amplitude of the $\mathrm{N} 2$ component or a decline, relative to a value of one. Our results confirm that cathodal tDCS significantly reduced the amplitude of the N2 component when compared to the control experiment. The pergolide medication prolonged this effect for up to 24 hours. The symbols indicate significant differences between the pergolide and placebo medications $(\mathbf{0})$ or differences between time points $(*)$. 
significantly correlates with increasing pain sensation. ${ }^{35}$

In a recent study, the effect of tDCS has been investigated by PET. ${ }^{13}$ Concerning painrelated regions, cathodal tDCS significantly diminished regional cerebral blood flow in the right ACC and the right thalamus. As the ACC is widely interconnected with primary and premotor areas, ${ }^{36}$ it is likely that the stimulation of the M1 could result in a secondary inhibition of the ACC, and as a consequence, in N2 amplitude reduction. The antinociceptive effect as revealed by the psychophysical experiment could reflect the diminished involvement of the ACC in pain processing. DC stimulation of the left motor cortex resulted in several critical changes at the contralateral side. ${ }^{13}$ Relative increases in regional cerebral blood flow after cathodal tDCS compared to sham tDCS were found in the right M1, frontal pole, primary sensorimotor cortex, and parietal occipital cortex. Regional CBF increase in homologous contralateral M1 was also found after rTMS to left M1. ${ }^{37,38}$ Our results suggest that the stimulation of the left motor cortex has an influence on LEP components and pain perception of both sides. However, a recent study from Le Pera et al. ${ }^{39}$ showed that the physiological activation of the motor cortex is able to reduce pain perception and LEP amplitude only when the motor area contralateral to painful stimuli is activated.

Relevant clinical studies show that repeatedly administering anodal tDCS over the M1 diminished pain sensation in patients with traumatic spinal cord injury ${ }^{40}$ and induced significantly greater pain reduction compared with sham stimulation in patients with fibromyalgia. ${ }^{41}$ However, comparing these results to our data, Fregni et al. ${ }^{40,41}$ found pain reduction after anodal tDCS. The divergent results can be explained by the difference between acute and chronic pain processing. Pathological changes due to chronic pain are characterized by many functional and structural changes in the brain $^{42,43}$ and these cortical reorganizations probably lead to changes in cortical excitability.

In our study, the oral administration of pergolide prolonged the effect of cathodal tDCS for up to 24 hours on LEPs. The possible mechanisms of DC-induced after-effect were investigated by several previous studies. Pharmacological intervention suggests that the after-effect is $\mathrm{N}$-methyl-D-aspartate (NMDA)receptor dependent. ${ }^{44-46}$ Dextromethorphan (NMDA-receptor and intracellular sigma 1 receptor blocker) intake prevented both anodal and cathodal tDCS-induced after-effects, demonstrating that dextromethorphan critically interferes with the functionality of tDCS, irrespective of the polarity of DC stimulation. ${ }^{44}$ It is known that long-lasting NMDA-receptor dependent cortical excitability and activity shifts are involved in neuroplastic modification. Dopaminergic mechanisms stabilize these processes, as shown by animal experiments. ${ }^{47-49}$ Dopamine (DA) resident in the synapses could strongly influence the induction of long-term potentiation and/or longterm depression through specific changes in the initial levels of cAMP and $\mathrm{Ca}^{2+}$, which are key regulators of LTPs in the hippocampus, striatum, and prefrontal cortex. ${ }^{50-53}$ One study showed that long-term potentiation dependent processes such as practice-dependent plasticity are enhanced by DA. ${ }^{54}$ DA acting on D1 receptors increases NMDA currents. ${ }^{55}$ In a recent study, the dopaminergic influence on NMDA receptor-dependent neuroplasticity was investigated using tDCS. The enhancement of D2, and to a lesser degree, of $\mathrm{D} 1$ receptors by pergolide consolidated tDCS-generated excitability diminution up until the morning poststimulation. ${ }^{17}$ Our results are in agreement with this study.

An antinociceptive effect of pergolide is implausible in the case of a single oral dose of $0.025 \mathrm{mg}$. Pronociceptive or antinociceptive effects of pergolide have not yet been published. However, the administration of levodopa, an indirect DA agonist, has been reported to reduce pain ratings in painful diabetic neuropathy in humans. ${ }^{56}$ The DA reuptake inhibitor bupropion also has analgesic effects. ${ }^{57}$ Contrary to these results, the systematic administration of DA D2 receptor antagonist in humans has also been shown to reduce pain ratings in clinical trials. ${ }^{58,59}$

The reduction of pain perception and the amplitudes of N2 and P2 components could also be due to a normal habituation observed by several studies. ${ }^{60,61}$ Therefore, we have repeated the measurements in the absence of tDCS and medication. Although during the control experiment a normal habituation process was observed (the N2 and P2 amplitudes 
were reduced insignificantly), our results demonstrated that medication conditions with tDCS induced a significant amplitude reduction of the N2 peak. In addition to this, the psychophysical evaluation did not reveal significant changes in subjective pain perception during the control experiment.

To summarize, we observed that following cathodal tDCS, the N2 amplitude of the LEP components were significantly decreased when compared to the control experiment, and simultaneously, pain sensation was reduced. The changes in LEPs remained stable for up to two hours after 15 minutes of cathodal stimulation and pergolide prolonged the effect of the cathodal tDCS, causing a decrease in the amplitude of the N2 component for up to 24 hours. Our findings were based on experimentally induced pain using LEPs in a population of healthy subjects. The limitation of our investigation is that results from a study using healthy subjects cannot be directly transferable to clinical settings. However, on the way toward a clinical application of either rTMS or tDCS, to our knowledge, this is the first study observing plasticity-prolonging effects of drugs affecting the CNS on a clinically relevant behavioral range. Two principle effects may be relevant in further clinical studies: prolongation of excitability enhancing effects for diseases such as stroke and Parkinson's disease by, for example, amphetamine $^{62,63}$ or d-cycloserine; ${ }^{64}$ and prolongation of inhibitory after-effects, as shown here, on pain, epilepsy, or other diseases associated with cortical hyperexcitability.

\section{References}

1. Tsubokawa T, Katayama Y, Yamamoto T, Hirayama T, Koyama S. Treatment of thalamic pain by chronic motor cortex stimulation. Pacing Clin Electrophysiol 1991;14:131-134.

2. Garcia-Larrea L, Frot M, Valeriani M. Brain generators of laser-evoked potentials: from dipoles to functional significance. Neurophysiol Clin 2003;33: 279-292.

3. Tamura Y, Okabe S, Ohnishi TN, et al. Effects of 1-Hz repetitive transcranial magnetic stimulation on acute pain induced by capsaicin. Pain 2004;107: 107-115.

4. Pridmore S, Oberoi G, Marcolin M, George M. Transcranial magnetic stimulation and chronic pain: current status. Australas Psychiatry 2005;13: 258-265.

5. Lefaucheur JP. New insights into the therapeutic potential of non-invasive transcranial cortical stimulation in chronic neuropathic pain. Pain 2006;122:11-13.

6. Fregni F, Freedman S, Pascual-Leone A. Recent advances in the treatment of chronic pain with noninvasive brain stimulation techniques. Lancet Neurol 2007;6:188-191.

7. Nitsche MA, Paulus W. Excitability changes induced in the human motor cortex by weak transcranial direct current stimulation. J Physiol 2000;527: 633-639.

8. Nitsche MA, Paulus W. Sustained excitability elevations induced by transcranial DC motor cortex stimulation in humans. Neurology 2001;57: 1899-1901.

9. Wassermann EM, Grafman J. Recharging cognition with DC brain polarization. Trends Cogn Sci 2005;9:503-505.

10. Poreisz Cs, Boros K, Antal A, Paulus W. Safety aspects of transcranial direct current stimulation concerning healthy subjects and patients. Brain Res Bull 2007;72:208-214.

11. Bindman LJ, Lippold OC, Redfearn JW. The action of brief polarizing currents on the cerebral cortex of the rat (1) during current flow and (2) in the production of long-lasting after-effects. J Physiol 1964;172:369-382.

12. Purpura DP, McMurtry JG. Intracellular activities and evoked potential changes during polarization of motor cortex. J Neurophysiol 1965;28: $166-185$.

13. Lang N, Siebner HR, Ward NS, et al. How does transcranial DC stimulation of the primary motor cortex alter regional neuronal activity in the human brain? Eur J Neursci 2005;22:495-504.

14. Nitsche MA, Schauenburg A, Lang N, et al. Facilitation of implicit motor learning by weak transcranial direct current stimulation of the primary motor cortex in the human. J Cogn Neurosci 2003;15:619-626.

15. Rogalewski A, Breitenstein C, Nitsche MA, Paulus W, Knecht S. Transcranial direct current stimulation disrupts tactile perception. Eur J Neurosci 2004;20:313-316.

16. Antal A, Nitsche MA, Paulus W. Transcranial direct current stimulation and the visual cortex. Brain Res Bull 2006;68:459-463.

17. Nitsche MA, Lampe C, Antal A, et al. Dopaminergic modulation of long-lasting direct currentinduced cortical excitability changes in the human motor cortex. Eur J Neurosci 2006;23:1651-1654.

18. Csifcsák G, Antal A, Hillers F, et al. Modulatory effect of transcranial direct current stimulation on acute pain perception [abstract]. Congress of the 
Federation of European Psychophysiological Societies, Hanover, Germany 2006;A-0118.

19. Deleu D, Northway MG, Hanssens Y. Clinical pharmacokinetic and pharmacodynamic properties of drugs used in the treatement of Parkinson's disease. J Pharmacokinet 2002;4:261-309.

20. Ziemann U, Bruns D, Paulus W. Enhancement of human motor cortex inhibition by the dopamine receptor agonist pergolide: evidence from transcranial magnetic stimulation. Neurosci Lett 1996;208: $187-190$.

21. Ziemann U, Tergau F, Bruns D, Baudewig J, Paulus W. Changes in human motor cortex excitability induced by dopaminergic and anti-dopaminergic drugs. Electroencephalogr Clin Neurophysiol 1997; 105:430-437.

22. Spiegel J, Hansen C, Treede RD. Clinical evaluation for the assessment of impaired pain sensitivity by thulium-laser evoked potentials. Clin Neurophysiol 1999;111:725-735.

23. Treede RD, Lorenz J, Baumgartner U. Clinical usefulness of laser-evoked potentials. Neurophysiol Clin 2003;33:303-314.

24. Snyder E, Hillyard SA. Long-latency evoked potentials to irrelevant, deviant stimuli. Behav Biol 1976;16:319-331.

25. Kenemans JL, Verbaten MN, Roelofs JW, Slangen JL. "Initial-" and "change-orienting reactions:" an analysis based on visual single-trial event-related potentials. Biol Psychol 1989;28:199-226.

26. Alho K, Winkler I, Escera C, et al. Processing of novel sounds and frequency changes in the human auditory cortex: magnetoencephalographic recordings. Psychophysiology 1998;35:211-224.

27. Escera C, Alho K, Winkler I, Naatanen R. Neuronal mechanisms of involuntary attention to acoustic novelty and change. J Cogn Neurosci 1998;10: 590-604.

28. Willer JC. Comparative study of perceived pain and nociceptive flexion reflex in man. Pain 1977; 3:69-80.

29. Creaćh C, Henry P, Caille JM, Allard M. Functional MR imaging analysis of pain-related brain activation after acute mechanical stimulation. Am J Neuroradiol 2000;21:1402-1406.

30. Frot M, Mauguière F. Dual representation of pain in the operculo-insular cortex in humans. Brain 2003;126:438-450.

31. Antal A, Nitsche MA, Paulus W. External modulation of visual perception in humans. Neuroreport 2001;12:3553-3555.

32. Antal A, Kincses ZT, Nitsche MA, Bártfai O, Paulus W. Excitability changes induced in the human primary visual cortex by transcranial direct current stimulation: direct electrophysiological evidence. Invest Ophthalmol Vis Sci 2004;45:702-707.
33. Peyron R, Laurent B, Garcia-Larrea L. Functional imaging of brain responses to pain. A review and meta-analysis. Neurophysiol Clin 1999;30: 263-288.

34. Iannetti GD, Zambreanu L, Cruccu G, Tracey I. Operculoinsular cortex encodes pain intensity at the earliest stages of cortical processing as indicated by amplitude of laser-evoked potentials in humans. Neuroscience 2005;131:199-208.

35. Büchel C, Bornhovd K, Quante M, et al. Dissociable neural responses related to pain intensity, stimulus intensity, and stimulus awareness within the anterior cingulate cortex: a parametric singletrial laser functional magnetic resonance resonance imaging study. J Neurosci 2002;22:970-976.

36. Devinsky O, Morrell MJ, Vogt BA. Contributions of anterior cingulate cortex to behaviour. Brain 1995;118:279-306.

37. Siebner HR, Peller M, Willoch F, et al. Lasting cortical activation after repetitive TMS of the motor cortex: a glucose metabolic study. Neurology 2000; 54:956-963.

38. Lee L, Siebner HR, Rowe JB, et al. Acute remapping within the motor system induced by lowfrequency repetitive transcranial magnetic stimulation. J Neurosci 2003;23:5308-5318.

39. Le Pera D, Brancucci A, De Armas L, et al. Inhibitory effect of voluntary movement preparation on cutaneous heat pain and laser-evoked potentials. Eur J Neurosci 2007;25:1900-1907.

40. Fregni F, Boggio PS, Lima MC, et al. A shamcontrolled, phase II trial of transcranial direct current stimulation for the treatment of central pain in traumatic spinal cord injury. Pain 2006;122: 197-209.

41. Fregni F, Gimenes R, Valle AC, et al. A randomized, sham-controlled, proof of principle study of transcranial direct current stimulation for the treatment of pain in fibromyalgia. Arthritis Rheum 2006; 54:3988-3998.

42. Grusser SM, Winter C, Muhlnickel W, et al. The relationship of perceptual phenomena and cortical reorganization in upper extremity amputees. Neuroscience 2001;102:263-272.

43. Apkarian AV, Sosa Y, Sonty S, et al. Chronic pain is associated with decreased prefrontal and thalamic gray matter density. J Neurosci 2004;24:1041010415 .

44. Liebetanz D, Nitsche MA, Tergau F, Paulus W. Pharmacological approach to the mechanisms of transcranial DC-stimulation-induced after-effects of human motor cortex excitability. Brain 2002;125: 2238-2247.

45. Nitsche MA, Fricke K, Henschke U, et al. Pharmacological modulation of cortical excitability shifts induced by transcranial direct current stimulation in humans. J Physiol 2003;553:293-301. 
46. Nitsche MA, Jaussi W, Liebetanz D, et al. Consolidation of externally induced human motor cortical neuroplasticity by d-cycloserine. Neuropsychopharmacology 2004;29:1573-1578.

47. Otani S, Blond O, Desce J-M, Crépel F. Dopamine facilitates long-term depression of glutamatergic transmission in rat prefrontal cortex. Neuroscience 1998;85:669-676.

48. Bailey CH, Giustetto M, Huang YY, Hawkins RD, Kandel RR. Is heterosynaptic modulation essential for stabilizing Hebbian plasticity and memory? Nat Rev Neurosci 2000;1:11-20.

49. Huda K, Salunga TL, Matsunami K. Dopaminergic inhibition of excitatory inputs onto pyramidal tract neurons in cat motor cortex. Neurosci Lett 2001;307:175-178.

50. Frey U, Huang YY, Kandel ER. Effects of cAMP stimulate a late stage of LTP in hippocampal CA1 neurons. Science 1993;260:1661-1664.

51. Jay TM, Gurden H, Yamaguchi T. Rapid increase in PKA activity during long-term potentiation in the hippocampal afferent fiber system to the prefrontal cortex in vivo. Eur J Neurosci 1998;10: 3302-3306.

52. Gurden H, Takita M, Jay TM. Essential role of D1 but not D2 receptors in the NMDA receptordependent long-term potentiation at hippocampalprefrontal cortex synapses in vivo. J Neurosci 2000; 20:RC106.

53. Spenser JP, Murphy KP. Activation of cyclic AMP-dependent protein kinase is required for long-term enhancement at corticostriatal synapses in rats. Neurosci Lett 2002;329:217-221.

54. Meintzschel F, Ziemann U. Modification of pratice-dependent plasticity in human motor cortex by neuromodulators. Cerebral Cortex 2006;16: 1106-1115.

55. Cepeda C, Colwell CS, Itri JN, Chandler SH, Levine MS. Dopaminergic modulation of NMDA- induced whole cell currents in neostriatal neurons in slices: contribution of calcium conductances. J Neurophysiol 1998;79:82-94.

56. Ertas M, Sagduyu A, Arac N, Ertekin C. Use of levodopa to relieve pain from painful symmetrical diabetic neuropathy. Pain 1998;75:275-279.

57. Semenchuk MR, Davis B. Efficacy of sustained release bupropion in neuropathic pain: an open-label study. Clin J Pain 2000;16:6-11.

58. Dundee JW, Love WJ, Moore JC. Alterations in response to somatic pain associated with anesthesia. XV. Further studies with phenothiazine derivates and similar drugs. Brit J Anaesth 1963;35:597-609.

59. Zitman FG, Linssen AC, Edelbroek PM, Van Kempen GM. Does addition of low-dose flupentixol enhance the analgetic effects of low-dose amitriptyline in somatoform pain disorder. Pain 1991;47: 25-30.

60. Weiss T, Kumpf K, Ehrhardt J, Gutberlet I, Miltner WH. A bioadaptive approach for experimental pain research in humans using laser-evoked brain potentials. Neurosci Lett 1997;227:95-98.

61. Valeriani M, de Tommaso M, Restuccia D, et al. Reduced habituation to experimental pain in migraine patients: a $\mathrm{CO}(2)$ laser evoked potential study. Pain 2003;105:57-64.

62. Galdstone DJ, Black SE. Enhancing recovery after stroke with noradrenergic pharmacotherapy: a new frontier? Can J Neurol Sci 2000;27:97-105.

63. Martinsson L, Eksberg S. Drugs for stroke recovery: the example of amphetamines. Drugs Aging 2004;21:67-79.

64. Nadeau SE, Wu SS. CIMT as behavioral engine in research on physiological adjuvants to neurorehabilitation: the challenge of merging animal and human research. NeuroRehabilitation 2006;21: $107-130$. 


\subsection{Gender-specific modulation of short-term neuroplasticity in the visual cortex induced by transcranial direct current stimulation}

Chaieb L, Antal A, Paulus W. Vis Neurosci. 2008; 25(1):77-81.

There is a well defined correlation between the effects of transcranial electric stimulation techniques and gender (Kuo et al., 2006). Here we retrospectively analysed data collected from previous investigation of the visual cortex: phosphene thresholds (PTs), visual evoked potentials (VEPs) and contrast sensitivity measurements (CSs) to explore whether there was any correlation between these indicators of visual cortex excitability and gender. Results suggest that cathodal tDCS- induced aftereffects were non- significant between genders, but anodal tDCS in females showed significantly enhanced excitability. This study indicates that sex differences do indeed exist between the genders where external stimulation is concerned. Primary influences may include the expression of different gonadal hormones in males and females. 


\title{
Gender-specific modulation of short-term neuroplasticity in the visual cortex induced by transcranial direct current stimulation
}

\author{
LEILA CHAIEB, ANDREA ANTAL, AND WALTER PAULUS \\ Department of Clinical Neurophysiology, Georg-August University, Göttingen, Germany
}

(Received August 30, 2007; AcCePted December 14, 2007)

\begin{abstract}
Transcranial direct current stimulation (tDCS) is a non-invasive method of modulating levels of cortical excitability. In this study, data gathered over a number of previously conducted experiments before and after tDCS, has been re-analyzed to investigate correlations between sex differences with respect to neuroplastic effects. Visual evoked potentials (VEPs), phosphene thresholds (PTs), and contrast sensitivity measurements (CSs) are used as indicators of the excitability of the primary visual cortex. The data revealed that cathodally induced excitability effects $10 \mathrm{~min}$ post stimulation with tDCS, showed no significant difference between genders. However, stimulation in the anodal direction revealed sex-specific effects: in women, anodal stimulation heightened cortical excitability significantly when compared to the age-matched male subject group. There was no significant difference between male and female subjects immediately after stimulation. These results indicate that sex differences exist within the visual cortex of humans, and may be subject to the influences of modulatory neurotransmitters or gonadal hormones which mirror short-term neuroplastic effects.
\end{abstract}

Keywords: Visual cortex, Neuroplasticity, tDCS, Gender-effects

\section{Introduction}

In the human brain, the visual and motor cortices continue to be popular models for the investigation of induced structural plasticity in the adult brain, as their effects can be measured by visual (VEPs and PTs) responses or motor evoked potentials (MEPs), respectively (Nitsche \& Paulus, 2000; Boroojerdi et al., 2002; Antal et al., 2004). There is growing evidence to suggest that neuroplastic changes are influenced by gender-specific pressures. Kuo et al. (2006) recently reported sex differences in the primary motor cortex after short-duration tDCS; female subjects showed prolonged after-effects of cathodal stimulation, while male subjects showed stronger anodally-induced after effects. Previous animal and human studies of neuroplasticity and sex differences centered on cognitive functions (Kimura, 2002; Juraska, 1998) where male and female populations are assessed for hormonal influences on cognitive functions (spatial navigation facial processing emotion and memory; (see Cahill, 2006 for review)), which are believed to underlie these differences in cognitive responses between the sexes.

Address correspondence and reprint requests to: Leila Chaieb, Department of Clinical Neurophysiology, Georg-August University, RobertKoch-Str. 40, 37075 Göttingen, Germany. E-mail: leilachaieb@med. uni-goettingen.de
Administration of polarizing currents to the rat cerebral cortex revealed the ability to induce prolonged changes in cortical excitability (Bindmann et al., 1964). In humans, changes in cortical excitability, induced by tDCS have been shown to precede more enduring plastic changes that are reversible and can be pharmacologically modulated (Lang et al., 2005; Nitsche et al., 2003b, 2006). Stimulation with tDCS applied to the motor cortex is polarity-dependent; anodal stimulation results in cortical excitation, while cathodal tDCS produces inhibition of activated cortical networks (Nitsche \& Paulus, 2000).

The visual cortex possesses the ability to undergo induced and spontaneous neuroplastic changes that have been demonstrated to lead to both short-term and, to potentiate, more permanent alterations at the synapse (Sherman \& Spear, 1982). tDCS over the primary visual cortex in an anodal direction reduces PTs, while stimulation in the cathodal direction reverses this effect (Antal et al., 2003). Also cathodal stimulation has an inhibitory effect on the N70 component of VEPs and anodal tDCS induces the reverse response. CS measurements also reflect cathodal effects (Antal et al., 2001). The duration of tDCS after-effects are able to outlast the stimulation period if administered for some minutes (Nitsche $\&$ Paulus, 2001) over the primary motor cortex; tDCS after-effects are not so enduring when applied to the primary visual cortex (Antal et al., 2004). Visual and motor cortices vary with regards to factors influencing neuroplasticity and the excitatory/inhibitory 
circuitries, gonadal sex hormones and neurotrophins which act upon them (Lund, 2002; Keller, 1993). Differences in cortical connections and neuronal membrane properties, including receptor expression, between the primary motor and visual cortices may also account for the contrasting responses to the application of tDCS.

In order to examine how neuroplasticity in the visual cortex is influenced, if at all by gender, data gathered from previous experiments involving tDCS modulation of PTs, VEPs, and CSs, were reviewed (Antal et al., 2001, 2003, 2004). Interactions between gender and tDCS stimulation were analyzed, in an attempt to reveal potential gender-bias in induced short-term neuroplastic effects.

\section{Materials and methods}

\section{Participants}

Data collected from previous experiments conducted with 46 healthy subjects, was re-analyzed to ascertain whether neuroplasticity is influenced by interactions with gender. Of the 46 subjects, 22 were male (average age 27.2 years, SD: \pm 5.4 ), and 24 were female (average age 23.6 years, SD: \pm 2.4 ). None of the participants were involved in more than one of the original studies. Participants were interviewed prior to the start of the experiment, about their state of health, and satisfied all the necessary conditions; no history of neurological or psychological disorders, metallic implants, drug abuse, or alcoholism. No subjects were taking regular medication at the time and during the experiment. All of the participants gave written and informed consent and all experiments were conducted in accordance with the Declaration of Helsinki, and with the approval of the ethics committee of the University of Goettingen, Germany.

tDCS was administered to all subjects for a 7 or $10 \mathrm{~min}$ period (Table 1), in both an anodal and cathodal direction, using a battery-driven constant current stimulator (Schneider Electronic, Gleichen, Germany). A pair of standard rubber electrodes $(5 \times$ $7 \mathrm{~cm}^{2}$ ) encased in saline-soaked synthetic sponges, were placed over the $\mathrm{Oz}$ (active electrode) and $\mathrm{Cz}$ (reference electrode) posi- tions. The applied current had an intensity of $1.0 \mathrm{~mA}$. Stimulation sessions were separated by one week to avoid interference between stimulations.

\section{Measurement of visual cortical excitability}

Three parameters were measured; PT, CS, and VEPs, as comparable indicators of visual cortical excitability (for technical details and protocols see Antal et al., 2001, 2003, 2004). Participants' PTs, VEPs, and CS were measured prior to, immediately after, and 10 min after cathodal and anodal tDCS stimulation. See Table 1 for a summary of measurements and subject characteristics.

\section{Statistics}

All data was standardized prior to analysis in order to gain a result relating to absolute change in cortical excitability (\% change) when compared to baseline activity prior to, immediately after, and $10 \mathrm{~min}$ post tDCS stimulation. Values over $100 \%$ are an increase in cortical excitability and represent an increase in visual sensitivity for CSs and an increase in amplitudes for VEPs, but a decrease in the threshold for the perception of phosphene (PTs).

Repeated measures ANOVA was performed on the calculated data dependent variable: \% change, within effects: STIMULATION (anodal, cathodal), TIME (0 and $10 \mathrm{~min}$ after), categorical factor: GENDER (male, female). Student's $t$-test (independent by GENDER) was used to explore significant differences between female and male subjects concerning anodal and cathodal post-stimulation values immediately after stimulation and $10 \mathrm{~min}$ post stimulation separately. Statistical significance was assumed at $p \leq 0.05$.

\section{Results}

Immediately after the anodal tDCS, both sexes appeared to be within the same range, however, at 10 min post-stimulation measurements, the female group still showed a strong facilitatory response, while the male group showed a marked inhibitory response. The means of male and female subjects have been tabulated, including stimulation direction, and duration (see Table 2).

Table 1. Stimulation paradigms and measurements ${ }^{\mathrm{a}}$

\begin{tabular}{|c|c|c|c|c|c|}
\hline Measurement & Sex & $\begin{array}{l}\mathrm{Age} \\
\mathrm{SD} \pm\end{array}$ & $\begin{array}{l}\text { Number of } \\
\text { participants }\end{array}$ & $\begin{array}{c}\text { tDCS } \\
\text { stimulation polarity }\end{array}$ & $\begin{array}{l}\text { Duration of } \\
\text { stimulation }\end{array}$ \\
\hline \multirow[t]{2}{*}{ Visual Evoked Potential (VEP) } & M & $\begin{array}{r}26.5 \\
2.7\end{array}$ & 10 & Anodal and Cathodal & $10 \mathrm{~min}$ \\
\hline & F & $\begin{array}{r}24.0 \\
2.0\end{array}$ & 5 & Anodal and Cathodal & $10 \mathrm{~min}$ \\
\hline \multirow[t]{2}{*}{ Stationary phosphene (PT) } & M & $\begin{array}{r}29.6 \\
7.8\end{array}$ & 4 & Anodal and Cathodal & $10 \mathrm{~min}$ \\
\hline & $\mathrm{F}$ & $\begin{array}{r}23.2 \\
2.4\end{array}$ & 12 & Anodal and Cathodal & $10 \mathrm{~min}$ \\
\hline \multirow[t]{2}{*}{ Contrast measurement (CS) } & M & $\begin{array}{r}26.8 \\
6.9\end{array}$ & 8 & Anodal and Cathodal & $7 \mathrm{~min}$ \\
\hline & $\mathrm{F}$ & $\begin{array}{r}23.8 \\
2.8\end{array}$ & 7 & Anodal and Cathodal & $7 \mathrm{~min}$ \\
\hline
\end{tabular}

${ }^{\text {a }}$ Summary of stimulation paradigms and measurements obtained from participants, including sex and age characteristics. Cathodal and anodal stimulation was applied to all subjects, and varied only in duration. For the VEP (10 male, 5 female) and stationary phosphene measurements (4 male, 13 female), stimulation in both polarities lasted $10 \mathrm{~min}$, whilst for the contrast measurements $(8$ male, 9 female $)$ stimulation lasted only $7 \mathrm{~min}$, and again in both polarities. 


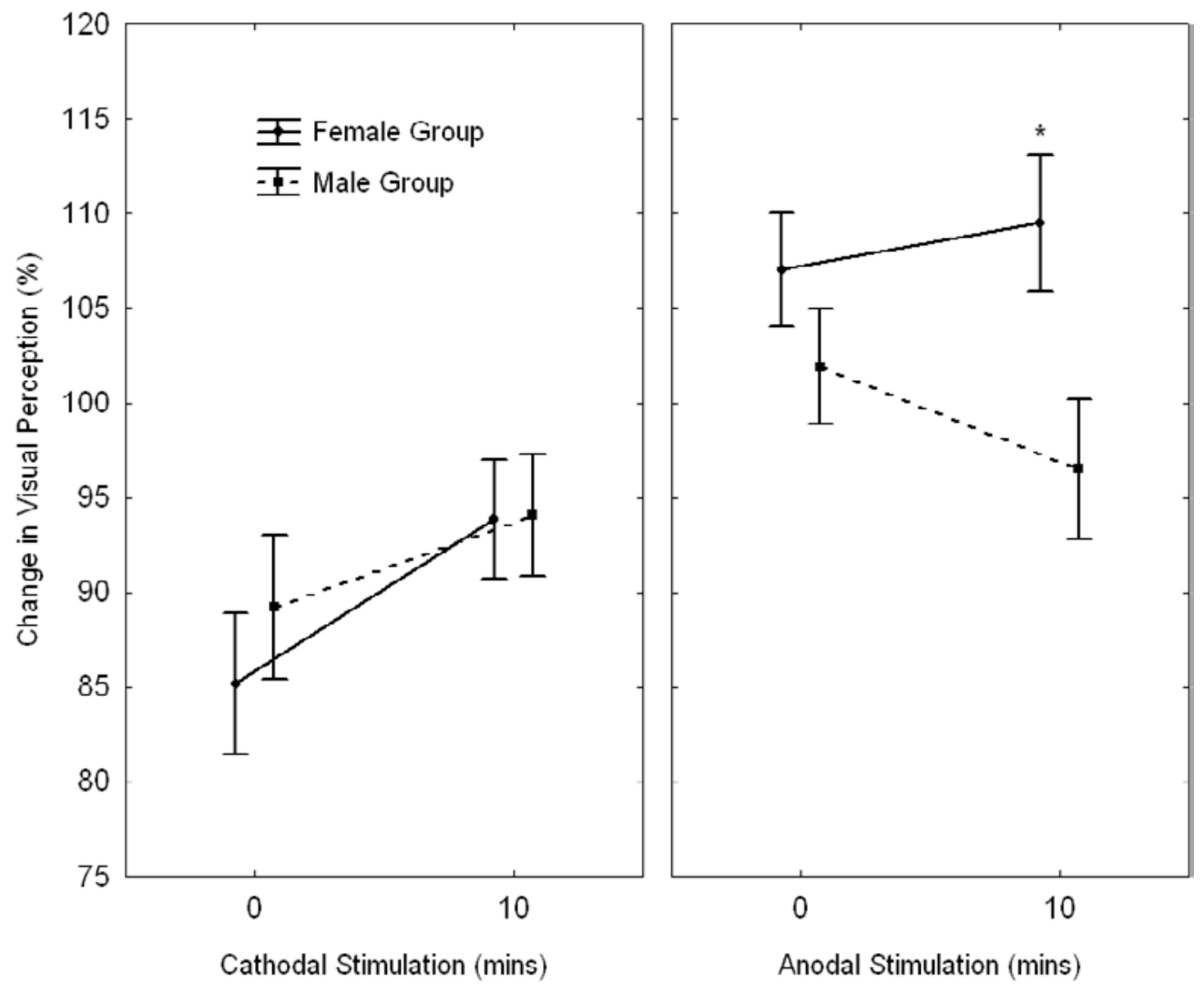

Fig. 1. Change in visual perception (\%) indicates all measurements (CS, PT, and VEPs), which are standardized and averaged. Anodal stimulation indicates an overall facilitation of all parameters in the female group. Cathodal stimulation shows no significant difference in effect between male and female subjects. The male group anodal tDCS results suggest an inhibition of all measurement parameters, increassing in effect after $10 \mathrm{~min}$ post-stimulation. Asterisks indicate results with a significant difference $(\rho \leq 0.05)$ between genders. $100 \%$ on the $\mathrm{x}$-axis is baseline; Error bars indicate SE.

The repeated measures ANOVA revealed a significant main effect of STIMULATION $(F(1,44)=28.8, p<0.0005)$, the interaction of GENDER and STIMULATION $(F(1,44)=5.1$, $p<0.05)$, and the interaction of STIMULATION and TIME $(F(1,44)=4.4, p<0.05)$ (see Table 3$)$.

The $t$-test did not reveal a significant difference between female and male subjects immediately after stimulation (cathodal stimulation: $t=-0.68, p=0.49, d f=44$; anodal stimulation $t=0.01$, $p=0.98, d f=44)$. Similarly, after $10 \mathrm{~min}$ post-stimulation for cathodal tDCS, there was no significant difference between the response of the male and female groups $(t=1,2, p=0.24, d f=$ $44)$, however, for anodal stimulation, a significant difference was observed $(t=2.54, p=0.01, d f=44)$ : female subjects were showing a higher facilitation than male subjects.

A one-way ANOVA was performed on the age variable, as this may have an influence on tDCS effects; there was no significant difference found between the two sexes, so age cannot be considered a factor in the differential response seen in tDCS-induced neuroplasticity.

\section{Discussion}

Our analysis showed that tDCS induced neuroplasticity in the human brain is partially influenced by gender-effects. No signifi-
Table 2. Means of \% change in cortical excitability with regard to the different tasks and stimulation direction ${ }^{\mathrm{a}}$

\begin{tabular}{lrr}
\hline \hline Stimulation direction & $\begin{array}{c}\text { Males } \\
\text { Mean }\end{array}$ & $\begin{array}{c}\text { Females } \\
\text { Mean }\end{array}$ \\
\hline CS & & \\
Anodal 0 & 101.7 & 100.6 \\
Cathodal 0 & 95.3 & 94.0 \\
Anodal 10 & 102.6 & 101.3 \\
Cathodal 10 & 99.7 & 100.3 \\
PTs & & \\
Anodal 0 & 109.5 & 109.2 \\
Cathodal 0 & 95.3 & 84.7 \\
Anodal 10 & 110.5 & 116.6 \\
Cathodal 10 & 96.2 & 94.0 \\
VEPs & & \\
Anodal 0 & 93.1 & 109.6 \\
Cathodal 0 & 71.8 & 104.9 \\
Anodal 10 & 85.9 & 85.0 \\
Cathodal 10 & 88.6 & \\
\hline \hline
\end{tabular}

${ }^{a}$ Means of $\%$ change in cortical excitability with regard to the different tasks and stimulation direction. Means for both measurements immediately and 10 min post-stimulation, for males and females are listed. Note that with respect to PTs an inverse calculation was used (See Materials and methods). 
Table 3. Results of repeated measures ANOVA for both anodal and cathodal stimulation directions, for 0 and $10 \mathrm{~min}$ post-stimulation $^{\text {a }}$

\begin{tabular}{lcl}
\hline \hline & F & $p$ \\
\hline Gender & 1.44 & 0.23 \\
Stimulation & 28.8 & 0.000 \\
Gender $\times$ Stimulation & 5.17 & 0.028 \\
Time & 1.36 & 0.25 \\
Gender $\times$ Time & 1.66 & 0.20 \\
Stimulation $\times$ Time & 4.61 & 0.04 \\
Gender $\times$ Stimulation $\times$ Time & 0.28 & 0.6
\end{tabular}

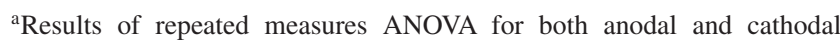
stimulation directions, for 0 and 10 min post-stimulation, for all parameters constituting "change in visual perception."

cant correlation between cathodal tDCS immediately after or 10 min post-stimulation was found; post-stimulation effects of anodal polarity were significantly different in the male versus female test groups. The female subjects immediately after and 10 min post-stimulation showed a facilitation of the stimulated cortical network. Males, however, showed an inhibition at the 10 min post-stimulation measurement. The tDCS induced inhibition or facilitation is a transient stage of induced short-term neuroplasticity closely related to changes in the state of the neuronal membrane. Ardolino et al. (2005) describe the potential mechanisms of tDCS in their paper examining the non-synaptic after-effects of DC stimulation on peripheral motor axons and neurons in the central nervous system. The migration of transmembrane proteins or changes in ion concentration across the membrane, in response to a continuous electrical field, may be responsible for the short-term neuroplastic effects; suggesting a possible mechanism of tDCS action on the human cortex. Further evidence can be derived from pharmacological studies also using tDCS. Nitsche et al. $(2003 b)$ demonstrated that the application of sodium and calcium ion channel blockers are able to attenuate both the immediate and longer term after-effects of anodal stimulation, while NMDA receptor blockers (glutamate) inhibit the development of long-term effects of tDCS, regardless of stimulation polarity (Liebetanz et al., 2002). These findings indicate that the short-term neuroplastic effects arise from alterations in neuronal membrane function and longer-term effects are mediated at the level of the synapse.

A similar study (Kuo et al., 2006) recently showed a reverse effect with respect to gender-bias, on MEPs measured from the primary motor cortex in males and females. The primary motor cortex relays information from the central nervous system to the periphery. However, the primary visual cortex is activated by input mechanisms, taking cues from the external environment, and relaying them to other cortical areas in order to be processed. Their cortical projections and efferents originate in and from differing neuroanatomical structures, and as a result, may possess contrasting neurotransmitter receptors. The origins of each of the parameters of visual perception are also differing and complex; VEPs are a measure of the cortical response to thalamic inputs, PTs indicate cortical background (V1-V2-V3) activity, while CSs mainly arise from the simultaneous activity of intracortical inhibitory processes at the spatial level of cortical receptive fields. By cross-analyzing each parameter, we were able to gain an overall effect of tDCS on visual perception, and use this to compare responses between the sexes. Therefore gender differences are related to a comparison between all parameters of visual perception between male and female groups.

This study suggests that there may exist, alternating pathways of neuroplasticity between the sexes in the visual cortex. The influences of gonadal sex hormones, neurotrophins, and the presence of neurotransmitter receptors on neural membranes may all be influential in the modulation of neuroplasticity, and may be expressed differently within each gender.

Inghilleri et al. (2004), using a repetitive transcranial magnetic stimulation (rTMS) paradigm, found that estrogens are able to influence cortical excitability; low estrogen levels have a damping effect on sodium channels which reduces the recruitment of excitatory interneurons, thus reducing cortical excitability, whereas at higher levels estrogens increase cortical excitability. In addition to this, Smith et al. $(2002 a, 2002 b)$ reported that progesterone has a reverse effect, and increases inhibition in neuronal networks by modulating GABAergic transmission; having earlier conducted one of the earliest studies to demonstrate menstrual cycle effects on cortical excitability (Smith et al., 1999). Studies in vitro have mirrored findings from in vivo investigations; Smith et al. (2002a, $2002 b$ ) examined fluctuations in gonadal hormone levels in organotypic hippocampal slices, in addition to using a kindling paradigm, and found that slices from intact males showed a greater level of baseline excitability than those of the female rats. They concluded that higher levels of testosterone may have an excitatory effect on hippocampal responses. In a recent review, Cooke and Woolley (2005) discuss the effect of gonadal steroids, androgen, and estrogen, on the morphology of dendrites forming synaptic connections. The pyramidal cells of the CA1 rat hippocampus undergo morphological changes in phase with the estrous cycle, suggesting that neuroplasticity on a synaptic level is influenced by fluctuations in steroid hormones. Earlier studies by Rudick and Woolley (2001) showed evidence of NMDA and $\mathrm{GABA}_{\mathrm{A}}$ estrogen mediated input to CA1 pyramidal cells, restoring the excitatory and inhibitory balance and increasing pyramidal cell responses to synaptic input.

If gonadal hormones are, on a molecular level, modulating synaptic connections, then the excitatory and inhibitory intracortical circuitry are also being modified. A study by Hausmann et al. (2006) investigated the effects of estradiol and progesterone on transcallosal inhibition. They concluded that these steroid hormones modulated the iSPs in accordance with each phase of the menstrual cycle, and that they were able to alter functional cerebral organization via activation of glutamatergic or GABAergic neurons. Maguire et al. (2005) demonstrated cycling expression of $\mathrm{GABA}_{\mathrm{A}}$ receptor subunits in hippocampal neurons mediating tonic inhibition during the ovarian cycle, altering seizure susceptibility and anxiety in mice.

Another intriguing aspect of gonadal hormones' involvement in neuromodulation is the rare incidences of migraine in males, in comparison to the statistical prevalence in females. A case review by Brandes (2006) discussed how the influence of estrogen on migraine is three-fold greater amongst women than men, and how the prevalence changes with reproductive status. The author also reports that menstrual migraine is pharmacologically resistant and without aura, whereas male migraineurs often experience aura even without headache.

In addition to differences in hormonal expression between the sexes, there appears to be evidence of differences in cortical structure. Recent studies have shown that in the rat visual cortex 
there exist dimorphic differences governed by gender. Nunez et al. (2002) showed that ovarian steroids expressed after postnatal day 20 reduce the number of neurons in the binocular region of the primary visual cortex of the female rat. Pakkenberg and Gundersen (1997) showed in an earlier study performed on human cadavers that on average females possess $16 \%$ fewer neocortical neurons than males, even though neuronal cortical density remains the same.

Understanding how the induction of short-term neuroplasticity in the mammalian cortex is achieved belies the understanding of neuronal excitability differences between males and females. The variation in background hormone levels and in cortical structure between males and females may account for the different observed responses to tDCS application. These findings, along with many other studies describing the influences of sex on plasticity, suggest that gender differences should be accounted for when evaluating changes in neuroplasticity, both short and long-term. However, more focused studies are required to elucidate the explicit mechanisms of gender-effects on neuroplasticity.

\section{Conclusion}

The current findings suggest that there exists gender-bias in the primary visual cortex which manifests itself in the altered excitability levels between the sexes resulting in a non-synaptic mechanism of neuromodulation in membrane function.

\section{References}

Antal, A., Kincses, T.Z., Nitsche, M.A., Bartfai, O. \& Paulus, W. (2004). Excitability changes induced in the human primary visual cortex by transcranial direct current stimulation: Direct electrophysiological evidence. Investigative Ophthalmology \& Visual Science 45, $702-707$.

Antal, A., Kincses, T.Z., Nitsche, M.A. \& Paulus, W. (2003). Manipulation of phosphene thresholds by transcranial direct current stimulation in man. Experimental Brain Research 150, 375-378.

Antal, A., Nitsche, M.A. \& Paulus, W. (2001). External modulation of visual perception in humans. NeuroReport 12, 3553-3555.

Ardolino, G., Bossi, B., Barberi, S. \& Priori, A. (2005). Non-synaptic mechanisms underlie the after-effects of cathodal transcutaneous direct current stimulation of the human brain. Journal of Physiology 568 , 653-663.

Bindmann, L.J., LiPPOLd, O.C.J. \& REDFEARN, J.W.T. (1964). The action of brief polarizing currents on the cerebral cortex of the rat (I) during current flow and (2) in the production of long-lasting after-effects. Journal of Physiology 172, 369-382.

Boroojerdi, B., Meister, I., Foltys, H., Sparing, R., Cohen, L. \& TöPPER, R. (2002). Visual and motor cortex excitability: A transcranial magnetic stimulation study. Clinical Neurophysiology 113, 1501-1504.

BRANDES, J.L. (2006). The influence of estrogen on migraine. JAMA 295, $1824-1830$.

CAHILl, L. (2006). Why sex matters for neuroscience. Nature Reviews Neuroscience 7, 477-484.

Cooke, B.M. \& Woolley, C.S. (2005). Gonadal hormone modulation of dendrites in the mammalian CNS. Neurobiology 64, 34-46.

Hausmann, M., Tegenthoff, M., Sänger, J., Janssen, F., Güntürkün, O. \& Schwenkreis, P. (2006). Transcallosal inhibition across the menstrual cycle: A TMS study. Clinical Neurophysiology 117, 26-32.
Inghilleri, M., Conte, A., Curra, A., Frasca, V., Lorenzano, C. \& Berardelli, A. (2004). Ovarian hormones and cortical excitability. An rTMS study in humans. Clinical Neurophysiology 115, 1063-1068.

JuRASKA, J.M. (1998). Neural plasticity and the development of sex differences. Annual Review of Sex Research 9, 20-38.

Keller, A. (1993). Intrinsic synaptic organization of the motor cortex. Cerebral Cortex 3, 430-441.

Kimura, D. (2002). Sex hormones influence human cognitive pattern. Neuroendocrinology Letters 23, 67-77.

Kuo, M., Paulus, W. \& Nitsche, M.A. (2006). Sex differences in cortical neuroplasticity in humans. NeuroReport 17, 1703-1707.

Lang, N., Siebner, H.R., Ward, N.S., Lee, L., Nitsche, M.A., Paulus, W., Rothwell, J.C., Lemon, R. \& Frackowiak, R.S. (2005). How does transcranial DC stimulation of the primary motor cortex alter regional neuronal activity in the human brain? European Journal of Neuroscience 22, 495-504.

Liebetanz, D., Nitsche, M.A., Tergau, F. \& Paulus, W. (2002). Pharmacological approach to the mechanisms of transcranial DC-stimulationinduced after-effects of human motor cortex excitability. Brain 125, $2238-2247$.

LUND, J.S. (2002). Specificity and non-specificity of synaptic connections within mammalian visual cortex. Journal of Neurocytology 31, 203-209.

Maguire, J.L., Stell, B.M, Rafizadeh, M. \& Mody, I. (2005). Ovarian cycle-linked changes in $\mathrm{GABA}_{\mathrm{A}}$ receptors mediating tonic inhibition alter seizure susceptibility and anxiety. Nature Neuroscience 8, 797-804.

Nitsche, M.A. \& Paulus, W. (2000). Excitability changes induced in the human motor cortex by weak transcranial direct current stimulation. Journal of Physiology 52, 633-639.

Nitsche, M.A. \& Paulus, W. (2001). Sustained excitability elevations induced by transcranial DC motor cortex stimulation in humans. $\mathrm{Neu}$ rology 57, 1899-1901.

Nitsche, M.A., Fricke, K., Henscke, U., Schlitterlau, A., Liebetanz, D., Lang, N., Henning, S., Tergau, F. \& Paulus, W. (2003a) Pharmacological modulation of cortical excitability shifts induced by transcranial direct current stimulation in humans. Journal of Physiology 553, 293-301.

Nitsche, M.A., Lampe, C., Antal, A., Liebetanz, D., Lang, N., TerGaU, F. \& Paulus, W. (2006). Dopaminergic modulation of longlasting direct current-induced cortical excitability changes in the human motor cortex. European Journal of Neuroscience 23, 1651-1657.

Nitsche, M.A., Nitsche, M.S., Klein, C.C., Tergau, F., Rothwell, J.C. $\&$ PAulus, W. (2003b). Level of action of cathodal DC polarisation induced inhibition of the human motor cortex. Clinical Neurophysiology 114, 600-604.

Nunez, J.L., SoDhI, J. \& JuRAsKa, J.M. (2002). Ovarian hormones after postnatal day 20 reduce neuron number in the rat primary visual cortex. Journal of Neurobiology 52, 312-321.

Pakkenberg, B. \& Gundersen, H.J.G. (1997). Neocortical neuron number in humans: Effect of sex and age. The Journal of Comparative Neurology 384, 312-320.

Rudick, C.N. \& Woolley, C.S. (2001). Estrogen regulates functional inhibition of hippocampal CA1 pyramidal cells in the adult female rat. Journal of Neuroscience 21, 6532-6543.

Sherman, S.M. \& Spear, P.D. (1982). Organization of visual pathways in normal and visually deprived cats. Physiology Review 62, 738-855.

Smith, M.D., Jones, L.S. \& Wilson, M.A. (2002a). Sex differences in hippocampal slice excitability: Role of testosterone. Neuroscience $\mathbf{1 0 9}$, 517-530.

Smith, M.J., Adams, L.F., Schmidt, P.J., Rubinow, D.R. \& Wassermann, E.M. (2002 b). Effects of ovarian hormones on human cortical excitability. Annals of Neurology 51, 599-603.

Smith, M.J., Keel, J.C., Greenberg, B.D., Adams, L.F., Schmidt, P.J., Rubinow, D.A. \& Wasserman, E.M. (1999). Menstrual cycle effects on cortical excitability. Neurology 53, 2069-2072. 


\section{Chapter 3: Discussion and Concluding Remarks}

The application of transcranial electric stimulation techniques, as we currently utilize them is a relatively new field of interest; however it is still a burgeoning one. The studies featured in the thesis intend to add to the already extensive knowledge of techniques used to induce enduring changes in the human cortex and to achieve longlasting LTP- or LTD- like effects. For example, tDCS has been shown to produce excitability increases or diminutions depending on its polarity, intensity and duration (Nitsche and Paulus, 2000, 2001, Nitsche et al., 2003). These effects have been wellcharacterised by the combined use of pharmacological agents and the performance of behavioural tasks during and after stimulation (Liebetanz et al., 2002, Antal et al., 2007).

While tDCS, rTMS and its patterned variant iTBS have been more extensively used, oscillating currents may provide a better insight into how cortical networks are synchronized and how, in turn, remote areas of the cortex communicate to form coherent patterns which contribute to the consolidation of learning and memory. The cortex is dynamic and so neuronal modulations and the potentiation of receptors and neurotransmitter cascades are all markers of induced LTP- like effects after application of tDCS or rTMS. For example tRNS and tACS may possess the ability to functionally connect rhythmic cortical networks in such a way that enables the long-range synchronization of neural populations. While we have postulated as to the mechanisms governing the mechanism of action of tRNS and tACS, we have yet to evaluate the mode of action both at the synaptic and network levels.

The aims of my $\mathrm{Ph} . \mathrm{D}$. thesis were to examine the response of the living human motor cortex to external transcranially applied currents; tRNS and tACS, and to evaluate whether these novel stimulation methods or techniques may be transposed or optimized to be used as potential therapeutic measures for the symptoms of pharmacologically intractable neurological disorders. Here I can report that we have demonstrated that a 
number of novel stimulation methods have robust and reproducible aftereffects when applied for a short duration, at a low stimulation intensity. The outcomes of my thesis work are as follows:

1.- We have successfully demonstrated that high- frequency oscillating currents (tRNS and tACS (including low range high- frequency stimulation within the kilohertz range) can induce neuroplastic aftereffects outlasting the duration of stimulation, when applied at low intensities to the human primary motor cortex.

2. - We have also applied these novel stimulation techniques to behavioural paradigms and examined their effects using fMRI. Results suggest that tRNS is able to modulate neuroplastic and cognitive processes in that intact human brain during the performance of both simple (finger- tapping task) and a more complex behavioural paradigm (SRTT). We have also demonstrated that the efficacy of high- frequency oscillating currents (tRNS) is dependent upon stimulation duration.

3. - We have successfully shown that application of transcranial sinusoidal currents in the low $\mathrm{kHz}$ range is able to induce robust changes in cortical excitability as measured using TMS. Recent evidence also suggests that this type of stimulation may have potentially beneficial therapeutic effects in patients with Parkinson's disease and associated symptoms (unpublished data). tACS has also been shown to have quantitative and long lasting-effects both in the motor and visual cortices. The efficacy of novel transcranial stimulation techniques may also be subject to genetic variations within the population, as we have demonstrated in our retrospective study of BDNF genotypes. These effects are also modulated by gender, as sex hormones have also been shown to play a role in responsiveness towards applied electrical currents.

Behavioural data goes a long way in providing evidence that tRNS and especially, tACS can alter neural communication by virtue of the implementing of the cognitive and motoric task: the variant of the SRTT. Task- related modulations of the aftereffects of tRNS and tACS also demonstrate that the cortex is responsive and reacts dynamically to external currents inducing transient but meaningful alterations in excitability. It would be of utmost interest to ascertain whether tRNS or high- frequency tACS is able to 
induce any modulations in cortical excitability in the pathological brain state, for example, Parkinson's disease. It is notoriously difficult to induce any lasting effects within the cortex that has been plastically altered and that possesses a neurochemical deficit. However, with such novel techniques, it is becoming increasingly likely that high- frequency oscillating currents will be the method of choice when investigating the effects of an application of a blanket electrical field applied to both cell cultures and the living cortex (Radman et al., 2007), as one can not only examine alterations in structures at the membrane and synaptic levels, but also the interactions within dynamic neuronal populations themselves.

Like most other transcranial techniques, tRNS and tACS are intensity and duration dependent, and so longer stimulation durations may recruit differing mechanisms that contribute to the observed excitatory aftereffects. Studies using varying durations of tDCS have already shown that these effects are markers of different stages of plasticity induction (Nitsche et al., 2003). Intra- DC effects, using short stimulation durations, have differing mechanisms of plasticity induction than those of longer stimulation durations. It is also relevant to discuss the wide variety of plasticities when considering the modes of action of novel stimulation techniques. For example, short-term plasticity in comparison to long-term LTP-like plastic effects is distinct due to a number of different factors; for example, short-term potentiation does not indicate a change in synaptic strength or weight. This is also the case when describing homeostatic plasticity and the Bienenstock-Cooper-Munro rule (Bienenstock et al., 1982), which may become more relevant when the mechanisms of oscillating currents are better characterised both in vitro and in the living cortex; the notion of applying currents that have both local and remote effects and that can potentially synchronise neuronal populations will have many evolutions of plasticity induction.

In addition to the aim of investigating basic mechanisms of neuroplasticity and neuroplastic induction, with regard to tRNS and tACS, this thesis also aims to underline the influence of genetic background on the induction of neuroplastic aftereffects using various stimulation techniques. Recent studies have highlighted the role of genetic variation within the population and it how it impacts upon how well both healthy and 
patient populations respond to the application of electrical and magnetic stimulation (Cheeran et al., 2009, Foltynie et al., 2009). The role of brain- derived neurotrophic factor (BDNF) may play a vital role in how transcranial stimulation techniques can be optimized for individual therapies. Further to this, the insight into why some groups of a particular genetic background are better responders or show less plasticity induction than others, goes some way into helping to find out the basic mechanisms of neuroplastic effects, and how they are achieved.

Other studies highlighted show how versatile transcranial electrical stimulation methods are, and how they enable us to gain useful insights into structure- function relationships within the human brain. For example, the frequency-dependent modulation of phosphene perception and the combined application of tDCS and pergolide illustrate that transcranial techniques can successfully modulate both focally stimulated areas of the cortex and yet still have remote effects.

\section{Concluding Remarks}

In summary the studies included within this thesis aim to demonstrate the identification of two novel methods of transcranial electric stimulation (tRNS and tACS) and to describe their distinctions as well as highlight their similarities to other more widely used techniques. The basic neurophysiology measures show that tRNS and tACS have positive effects on the cortex, while further studies aimed to optimize stimulation parameters and evaluate aftereffects. Studies focusing on high- frequency stimulation highlight the potential of next generation stimulation techniques, showing that although they possess the capability to induce sustainable aftereffects, they can be applied painlessly and safely. Finally, a retrospective data analysis on the visual cortex demonstrates the effect of gender and how it is an important factor that not only predisposes male and females to neurological and cognitive disturbances later in life, but how sex differences can create different responses to stimulation within a healthy human population. 
What these studies hope to provide is an improved knowledge of how novel techniques are identified and characterised and how they can be optimized to induce long- lasting neuroplastic aftereffects which, in turn, enable us to gain a better understanding of the mechanisms of neuroplasticity in the human cortex and the possibility to further utilize these techniques within a clinical setting. 


\section{References}

Ambrus GG, Paulus W, Antal A (2010) Cutaneous perception thresholds of electrical stimulation methods: comparison of tDCS and tRNS. Clin Neurophysiol 121:1908-1914.

Antal A, Boros K, Poreisz C, Chaieb L, Terney D, Paulus W (2008) Comparatively weak after-effects of transcranial alternating current stimulation (tACS) on cortical excitability in humans. Brain Stimul 1:97-105.

Antal A, Paulus W (2008) Transcranial direct current stimulation and visual perception. Perception 37:367-374.

Antal A, Terney D, Kuhnl S, Paulus W (2010) Anodal transcranial direct current stimulation of the motor cortex ameliorates chronic pain and reduces short intracortical inhibition. J Pain Symptom Manage 39:890-903.

Antal A, Terney D, Poreisz C, Paulus W (2007) Towards unravelling task-related modulations of neuroplastic changes induced in the human motor cortex. Eur $\mathbf{J}$ Neurosci 26:2687-2691.

Barker AT, Jalinous R, Freeston IL (1985) Non-invasive magnetic stimulation of human motor cortex. Lancet 1:1106-1107.

Berardelli A, Inghilleri M, Rothwell JC, Romeo S, Curra A, Gilio F, Modugno N, Manfredi M (1998) Facilitation of muscle evoked responses after repetitive cortical stimulation in man. Exp Brain Res 122:79-84.

Bienenstock EL, Cooper LN, Munro PW (1982) Theory for the development of neuron selectivity: orientation specificity and binocular interaction in visual cortex. $\mathrm{J}$ Neurosci 2:32-48.

Bliss TV, Lomo T (1973) Long-lasting potentiation of synaptic transmission in the dentate area of the anaesthetized rabbit following stimulation of the perforant path. J Physiol 232:331-356.

Brasil-Neto JP, Cohen LG, Pascual-Leone A, Jabir FK, Wall RT, Hallett M (1992) Rapid reversible modulation of human motor outputs after transient deafferentation of the forearm: a study with transcranial magnetic stimulation. Neurology 42:1302-1306.

Bromm B (1968) [Sodium rectification in the subthreshold excitation as computed from the voltage clamp analysis]. Pflugers Arch 302:233-244.

Butefisch CM (2006) Neurobiological bases of rehabilitation. Neurol Sci 27 Suppl $1:$ S18-23.

Castro-Alamancos MA, Rigas P, Tawara-Hirata Y (2007) Resonance (approximately 10 $\mathrm{Hz}$ ) of excitatory networks in motor cortex: effects of voltage-dependent ion channel blockers. J Physiol 578:173-191.

Chaieb L, Antal A, Paulus W (2010) Transcranial alternating current stimulation in the low $\mathrm{kHz}$ range increases motor cortex excitability. Restorative Neurology and Neuroscience In press.

Chaieb L, Kovacs G, Cziraki C, Greenlee M, Paulus W, Antal A (2009) Short-duration transcranial random noise stimulation induces blood oxygenation level 
dependent response attenuation in the human motor cortex. Exp Brain Res 198:439-444.

Cheeran B, Talelli P, Mori F, Koch G, Suppa A, Edwards M, Houlden H, Bhatia K, Greenwood R, Rothwell JC (2008) A common polymorphism in the brainderived neurotrophic factor gene (BDNF) modulates human cortical plasticity and the response to rTMS. J Physiol 586:5717-5725.

Cheeran BJ, Ritter C, Rothwell JC, Siebner HR (2009) Mapping genetic influences on the corticospinal motor system in humans. Neuroscience 164:156-163.

Citri A, Malenka RC (2008) Synaptic plasticity: multiple forms, functions, and mechanisms. Neuropsychopharmacology 33:18-41.

Cohen LG, Ziemann U, Chen R, Classen J, Hallett M, Gerloff C, Butefisch C (1998) Studies of neuroplasticity with transcranial magnetic stimulation. J Clin Neurophysiol 15:305-324.

Cooke S, Bliss T (2006) Plasticity in the human central nervous system. Brain 129:1659-1673.

Di Lazzaro V, Oliviero A, Profice P, Saturno E, Pilato F, Insola A, Mazzone P, Tonali $\mathrm{P}$, Rothwell JC (1998) Comparison of descending volleys evoked by transcranial magnetic and electric stimulation in conscious humans. Electroencephalogr Clin Neurophysiol 109:397-401.

Duffau H (2006) Brain plasticity: from pathophysiological mechanisms to therapeutic applications. J Clin Neurosci 13:885-897.

Feldman DE (2009) Synaptic mechanisms for plasticity in neocortex. Annu Rev Neurosci 32:33-55.

Figurov A, Pozzo-Miller LD, Olafsson P, Wang T, Lu B (1996) Regulation of synaptic responses to high-frequency stimulation and LTP by neurotrophins in the hippocampus. Nature 381:706-709.

Foltynie T, Cheeran B, Williams-Gray CH, Edwards MJ, Schneider SA, Weinberger D, Rothwell JC, Barker RA, Bhatia KP (2009) BDNF val66met influences time to onset of levodopa induced dyskinesia in Parkinson's disease. J Neurol Neurosurg Psychiatry 80:141-144.

Francis JT, Gluckman BJ, Schiff SJ (2003) Sensitivity of neurons to weak electric fields. J Neurosci 23:7255-7261.

Fritsch B, Reis J, Martinowich K, Schambra HM, Ji Y, Cohen LG, Lu B (2010) Direct current stimulation promotes BDNF-dependent synaptic plasticity: potential implications for motor learning. Neuron 66:198-204.

Gartside IB (1968) Mechanisms of sustained increases of firing rate of neurones in the rat cerebral cortex after polarization: role of protein synthesis. Nature 220:383384.

Hallett M (2000) Transcranial magnetic stimulation and the human brain. Nature 406:147-150.

Hebb D (1949) The Organisation of Behaviour. New York: Wiley.

Hess G, Donoghue J (1994) Long-term potentiation of horizontal connections provides a mechanism to reorganize cortical motor maps. J Neurophysiol 71:2543-2547.

Huang Y, Edwards M, Rounis E, Bhatia K, Rothwell J (2005) Theta burst stimulation of the human motor cortex. Neuron 45:201-206. 
Huang YZ, Chen RS, Rothwell JC, Wen HY (2007) The after-effect of human theta burst stimulation is NMDA receptor dependent. Clin Neurophysiol 118:10281032.

Huerta PT, Volpe BT (2009) Transcranial magnetic stimulation, synaptic plasticity and network oscillations. J Neuroeng Rehabil 6:7.

Islam N, Aftabuddin M, Moriwaki A, Hattori Y, Hori Y (1995) Increase in the calcium level following anodal polarization in the rat brain. Brain Res 684:206-208.

Jacobs KM, Donoghue JP (1991) Reshaping the cortical motor map by unmasking latent intracortical connections. Science 251:944-947.

Jalinous R (1991) Technical and practical aspects of magnetic nerve stimulation. J Clin Neurophysiol 8:10-25.

Jefferys JG (1995) Nonsynaptic modulation of neuronal activity in the brain: electric currents and extracellular ions. Physiol Rev 75:689-723.

Kanai R, Chaieb L, Antal A, Walsh V, Paulus W (2008) Frequency-dependent electrical stimulation of the visual cortex. Curr Biol 18:1839-1843.

Kobayashi M, Pascual-Leone A (2003) Transcranial magnetic stimulation in neurology. Lancet Neurol 2:145-156.

Kujirai T, Caramia MD, Rothwell JC, Day BL, Thompson PD, Ferbert A, Wroe S, Asselman P, Marsden CD (1993) Corticocortical inhibition in human motor cortex. J Physiol 471:501-519.

Kuo MF, Paulus W, Nitsche MA (2006) Sex differences in cortical neuroplasticity in humans. Neuroreport 17:1703-1707.

Liebetanz D, Nitsche M, Tergau F, Paulus W (2002) Pharmacological approach to the mechanisms of transcranial DC-stimulation-induced after-effects of human motor cortex excitability. Brain 125:2238-2247.

Lu P, Jones LL, Tuszynski MH (2005) BDNF-expressing marrow stromal cells support extensive axonal growth at sites of spinal cord injury. Exp Neurol 191:344-360.

Malenka R, Bear M (2004) LTP and LTD: an embarrassment of riches. Neuron 44:5-21.

Merton PA, Morton HB (1980) Stimulation of the cerebral cortex in the intact human subject. Nature 285:227.

Moliadze V, Antal A, Paulus W (2010a) Boosting brain excitability by transcranial high frequency stimulation in the ripple range. Under review in J Physiol.

Moliadze V, Antal A, Paulus W (2010b) Electrode-distance dependent after-effects of transcranial direct and random noise stimulation with extracephalic reference electrodes. Clin Neurophysiol.

Nissen, Bullmer (1987) Attentional requirements of learning: Evidence from performance measures. Cognitive Psychology 19:1-32.

Nitsche M, Nitsche M, Klein C, Tergau F, Rothwell J, Paulus W (2003) Level of action of cathodal DC polarisation induced inhibition of the human motor cortex. Clin Neurophysiol 114:600-604.

Nitsche M, Paulus W (2000) Excitability changes induced in the human motor cortex by weak transcranial direct current stimulation. J Physiol 527 Pt 3:633-639.

Nitsche M, Paulus W (2001) Sustained excitability elevations induced by transcranial DC motor cortex stimulation in humans. Neurology 57:1899-1901.

Nitsche M, Seeber A, Frommann K, Klein C, Rochford C, Nitsche M, Fricke K, Liebetanz D, Lang N, Antal A, Paulus W, Tergau F (2005) Modulating 
parameters of excitability during and after transcranial direct current stimulation of the human motor cortex. J Physiol.

Nitsche MA, Paulus W (2009) Noninvasive brain stimulation protocols in the treatment of epilepsy: current state and perspectives. Neurotherapeutics : the journal of the American Society for Experimental NeuroTherapeutics 6:244-250.

Pascual-Leone A, Amedi A, Fregni F, Merabet L (2005) The plastic human brain cortex. Annu Rev Neurosci 28:377-401.

Paulus W (2010) On the difficulties of separating retinal from cortical origins of phosphenes when using transcranial alternating current stimulation (tACS). Clin Neurophysiol 121:987-991.

Pogosyan A, Gaynor LD, Eusebio A, Brown P (2009) Boosting cortical activity at Betaband frequencies slows movement in humans. Curr Biol 19:1637-1641.

Purpura DP, McMurtry JG (1965) Intracellular activities and evoked potential changes during polarization of motor cortex. J Neurophysiol 28:166-185.

Radman T, Datta A, Peterchev AV (2007) In vitro modulation of endogenous rhythms by AC electric fields: Syncing with clinical brain stimulation. J Physiol 584:369370.

Reis J, Robertson EM, Krakauer JW, Rothwell J, Marshall L, Gertoff C, Wassermann EM, Pascual-Leone A, Hummel F, Celnik PA, Classen J, Floel A, Ziemann U, Paulus W, Siebner HR, Born J, Cohen LG (2008) Consensus: Can transcranial direct current stimulation and transcranial magnetic stimulation enhance motor learning and memory formation? Brain Stimul 1:363-369.

Ridding MC, Rothwell JC (2007) Is there a future for therapeutic use of transcranial magnetic stimulation? Nat Rev Neurosci 8:559-567.

Rossini PM, Barker AT, Berardelli A, Caramia MD, Caruso G, Cracco RQ, Dimitrijevic MR, Hallett M, Katayama Y, Lucking CH, et al. (1994) Non-invasive electrical and magnetic stimulation of the brain, spinal cord and roots: basic principles and procedures for routine clinical application. Report of an IFCN committee. Electroencephalogr Clin Neurophysiol 91:79-92.

Rothwell J (2005) Transcranial Electrical and Magnetic Stimulation of the Brain: Physiological Mechanisms. In: Magnetic stimulation in clinical neurophysiology(Chokroverty, M. H. a. S., ed), pp 43-60: Elsevier.

Rothwell JC (1997) Techniques and mechanisms of action of transcranial stimulation of the human motor cortex. J Neurosci Methods 74:113-122.

Ruohonen J, Ilmoniemi RJ (1999) Modeling of the stimulating field generation in TMS. Electroencephalogr Clin Neurophysiol Suppl 51:30-40.

Sanes J, Donoghue J (2000) Plasticity and primary motor cortex. Annu Rev Neurosci 23:393-415.

Schoen I, Fromherz P (2008) Extracellular stimulation of mammalian neurons through repetitive activation of $\mathrm{Na}+$ channels by weak capacitive currents on a silicon chip. J Neurophysiol 100:346-357.

Schutter DJ, Hortensius R (2010) Retinal origin of phosphenes to transcranial alternating current stimulation. Clin Neurophysiol 121:1080-1084.

Siebner HR, Lee L, Bestmann S (2003) Interleaving TMS with functional MRI: now that it is technically feasible how should it be used? Clin Neurophysiol 114:1997-1999. 
Siebner HR, Rothwell J (2003) Transcranial magnetic stimulation: new insights into representational cortical plasticity. Exp Brain Res 148:1-16.

Terney D, Chaieb L, Moliadze V, Antal A, Paulus W (2008) Increasing human brain excitability by transcranial high-frequency random noise stimulation. J Neurosci 28:14147-14155.

Yoshii A, Constantine-Paton M (2010) Postsynaptic BDNF-TrkB signaling in synapse maturation, plasticity, and disease. Dev Neurobiol 70:304-322.

Zaghi S, de Freitas Rezende L, de Oliveira LM, El-Nazer R, Menning S, Tadini L, Fregni F (2010) Inhibition of motor cortex excitability with $15 \mathrm{~Hz}$ transcranial alternating current stimulation (tACS). Neurosci Lett 479:211-214.

Ziemann U (2003) Pharmacology of TMS. Suppl Clin Neurophysiol 56:226-231.

Ziemann U, Chen R, Cohen LG, Hallett M (1998a) Dextromethorphan decreases the excitability of the human motor cortex. Neurology 51:1320-1324.

Ziemann U, Hallett M, Cohen LG (1998b) Mechanisms of deafferentation-induced plasticity in human motor cortex. J Neurosci 18:7000-7007.

Ziemann U, Muellbacher W, Hallett M, Cohen LG (2001) Modulation of practicedependent plasticity in human motor cortex. Brain 124:1171-1181.

Ziemann U, Rothwell JC (2000) I-waves in motor cortex. J Clin Neurophysiol 17:397405. 


\section{Acknowledgements}

Firstly, praise be to ALLAH, who in His compassion and kindness has enabled me to complete this Ph.D. study; it is in His infinite knowledge that everything unfolds.

My very special, personal and wholehearted thanks go to Prof. Dr. Andrea Antal, without whom this work would have been an impossible task. Her endless patience and unfailing enthusiasm have been a constant source of motivation and inspiration. I thank you; my mentor and my friend.

I would like to acknowledge the support and innovative vision of Prof. Dr. Walter Paulus, whose scientific insight has lead us far and on whose guidance we continually depend. I thank you for your kind support and tireless enthusiasm over these years.

I would like to express my thanks to Prof. Dr. Tobias Moser and to Prof. Dr. Florentin Wörgötter, members of my Ph.D. thesis committee, for their kind support and guidance during my studies.

I would also like to thank Frau Marion Kurze, my colleagues at the Department of Clinical Neurophysiology and all those who have generously given their time and patience to participate in our studies; it has been a pleasure working with each and every one of you.

This work would not have been possible without the endless and careful encouragement, guidance, support and love of my parents, Sandra and Kamel Chaieb, without whom I could have achieved nothing. It is a privilege to share this with you. My heartfelt thanks and love also go to my grandparents, Les and Marjorie Wiseman, and to my brother, Samir Chaieb, who have been a constant source of motivation and love in my life and memory.

My sincere thanks and love go to the many people that I have met during this course of study and whom I now have the privilege to call my friends: to Daniella Terney for making Goettingen and Hungary unforgettable; to Sandra Willinghoeffer and Stephanie Stiegel, Holger Rothkegel, Géza Gergely Ambrus, Zsuzanna G. Nagy, Min-Fang Kuo, Shane Fresnoza, Jessica Szturmann and Maren Schultz. You have all made my time in Goettingen enjoyable and worthwhile.

To Rachel Barratt, whose constant friendship has endured over time and distance, I express my love and thanks.

Last, but not by any means least, my personal thanks and love go to Manuel Hewitt; a best friend if ever there was one. 


\section{U R R I C U L U M V I T A E}

Name: Leila Chaieb

Date of Birth: September 23, 1982

Place of Birth: Sousse, Tunisia

Nationality: Tunisian

\section{Contact Details:}
Abteilung Klinische Neurophysiologie
Robert-Koch-Str.40
Göttingen
37075
$\mathrm{DE}$

EDUCATION

January 2007- current; reading for a Ph.D. in Neuroscience at the Georg- August University, Goettingen, Germany; graduate program: Sensory and Motor Neuroscience, Göttingen Graduate School for Neurosciences and Molecular Biosciences (GGNB), Georg-August-Universtity, Göttingen, Germany

September 2004- September 2005; read MSc Neuroscience at University College London, United Kingdom; Thesis: Analysis of Bipolar Disorder Drug Targets in a Model System- Phospholipase A2 Signalling.

September 2001- June 2004; read BSc (Hons) Neuroscience at the Victoria University of Manchester, United Kingdom; Thesis: Circadian Studies of Mice Expressing GFP and Per1. Dissertation: The Role of the Amygdala in Emotion. 
QUALIFICATIONS

Degrees: MSc Neuroscience

BSc (Hons) Neuroscience

CONFERENCES, ACTIVITIES AND PRESENTATIONS

2007-current- Supervision and teaching of students from the Medical Faculty of the Georg-August University, Goettingen.

2008-2009- Supervision and teaching of students from the ENI Neuroscience Program.

February 2007, 2008, 2009 and 2010- NWG Workshop on Basic and Advanced Transcranial Stimulation Methods at the Introduction to Transcranial Stimulation Methods Conference, Göttingen, Germany. Poster and oral presentations.

October 2008- NWG Workshop on Basic and Advanced Transcranial Stimulation Methods at the Third International Conference on Transcranial Magnetic and Direct Current Stimulation, Göttingen, Germany. Poster presentation.

August 2009- Student symposium at the Institute for Musicphysiology and Music medicine, Hannover, Germany. Oral presentation.

September 2010- 7th Annual ECNS / ISNIP Conference on Multimodal Brain Imaging in Neuropyschiatry, Istanbul, Turkey. Oral presentation. 
Chaieb L, Kovacs G, Cziraki C, Greenlee M, Paulus W, Antal A. Short-duration transcranial random noise stimulation induces blood oxygenation level dependent response attenuation in the human motor cortex. Exp Brain Res. 2009; 198(4):439-44.

Chaieb L, Antal A, Paulus W. Gender-specific modulation of short-term neuroplasticity in the visual cortex induced by transcranial direct current stimulation. Vis. Neurosci. 2008;25(1):77-81.

Chaieb L, Antal A, Paulus W. Evaluating Aftereffects of Transcranial Random Noise Stimulation on Cortical Excitability. (under review in Neural Plasticity).

Chaieb L, Antal A, Paulus W. Human motor cortex excitability can be modulated using combined tRNS and pharmacological intervention. (in preparation).

Chaieb L, Antal A, Paulus W. Transcranial alternating current stimulation in the low $\mathrm{kHz}$ range increases motor cortex excitability. (Restorative Neurology and Neuroscience; In press).

Terney D, Chaieb L, Moliadze V, Antal A, Paulus W. Increasing human brain excitability by transcranial high-frequency random noise stimulation. J Neurosci. 2008; 28(52):14147-55.

Kanai R, Chaieb L, Antal A, Walsh V, Paulus W. Frequency-dependent electrical stimulation of the visual cortex. Curr Biol. 2008;18(23):1839-43.

Antal A, Chaieb L, Moliadze V, Monte- Silva K, Poreisz C, Thirugnanasambandam N, Nitsche M, Shoukier M, Ludwig H, Paulus W. Brain-derived neurotrophic factor 
(BDNF) gene polymorphisms shape cortical plasticity in humans. Brain Stimulation 2010 .

Antal, A., Boros, K., Poreisz, C., Chaieb, L., Terney, D. \& Paulus, W. Comparatively weak after-effects of transcranial alternating current stimulation (tACS) on cortical excitability in humans Brain Stimulation 2008; 1:97-105.

Terney D, Bergmann I, Poreisz C, Chaieb L, Boros K, Nitsche MA, Paulus W, Antal A. Pergolide increases the efficacy of cathodal direct current stimulation to reduce the amplitude of laser-evoked potentials in humans. J Pain Symptom Manage. 2008; 36(1):79-91.

Göttingen, $8^{\text {th }}$ October 2010. 